\title{
A NARRATIVE STUDY OF THE EXPERIENCES OF PROVIDING CULTURALLY COMPETENT CARE BY FRONTLINE STAFF CARING FOR OLDER UKRAINIAN IMMIGRANTS IN A MONO-CULTURE LONG-TERM CARE FACILITY
}

\author{
by
}

\author{
Maryana Zaplatsinska, BScN, 2015 \\ Ryerson University, Toronto, Ontario, Canada \\ Master of Nursing Thesis \\ Presented to Ryerson University \\ In partial fulfillment of the \\ requirements for the degree of \\ Master of Nursing \\ In the Program of Nursing
}

Toronto, Ontario, Canada, 2015

(C) 2015 Maryana Zaplatsinska 


\section{AUTHOR'S DECLARATION}

I hereby declare that I am the sole author of this thesis. This is a true copy of the thesis, including any required final revisions, as accepted by my examiners.

I authorize Ryerson University to lend this thesis to other institutions or individuals for the purpose of scholarly research.

I further authorize Ryerson University to reproduce this thesis by photocopying or by other means, in total or in part, at the request of other institutions or individuals for the purpose of scholarly research.

I understand that my thesis may be made electronically available to the public. 


\begin{abstract}
A NARRATIVE STUDY OF THE EXPERIENCES OF PROVIDING CULTURALLY COMPETENT CARE BY FRONTLINE STAFF CARING FOR OLDER UKRAINIAN IMMIGRANTS IN A MONO-CULTURE LONG-TERM CARE FACILITY
\end{abstract} 2015

Maryana Zaplatsinska

Master of Nursing, Ryerson University

Frontline healthcare providers are increasingly called to demonstrate respect for clients' preferences by providing culturally competent care as part of person-centered approaches. Review of literature, however, shows culturally competent care practices have been poorly integrated into healthcare. Among Canada's population, care of older Ukrainian immigrants has received limited consideration. This narrative study explored experiences of two Ukrainian nurses providing culturally competent care in Ukrainian long-term care homes in the Greater Toronto Area. Textual and photographic data were analyzed via categorical-content and visual analysis approaches in keeping with narrative methodology. Three levels of data analysis were completed: emergent theme analysis, comparative theme analysis, and metaphoric representative analysis. Major themes include honoring the client, home is where the varenyky are served, the culturally competent nurse as the doorway to culturally competent care, and cultural insight as the solid foundation. Study implications are for organizational practice, policy and research. 


\section{ACKNOWLEDGEMENTS}

I would like to sincerely thank my supervisor, Dr. Lori Schindel Martin, for recognizing my capacity for growth and for the guidance along this journey. I am in awe of your deep wisdom, academic enthusiasm, and steady patience.

My thanks and appreciation to my Thesis Committee members, Dr. Mandana Vahabi and Dr. Charlotte Lee, who pointed out new directions and graciously shared their time.

I am so grateful to my participants for trusting me with their stories.

I would like to acknowledge the Ukrainian parishes in the Greater Toronto Area for their help with recruiting participants.

My heartfelt thanks go out to my loved ones, for their unwavering support. 


\section{DEDICATION}

Dedicated to Ukrainians everywhere. 


\section{TABLE OF CONTENTS}

AUTHOR'S DECLARATION ........................................................................................................ii

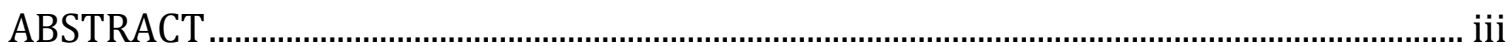

ACKNOWLEDGEMENTS ...................................................................................................................iv

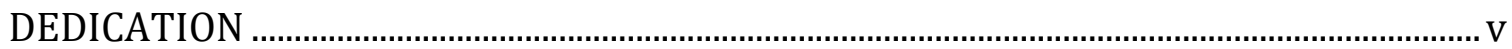

TABLE OF FIGURES ………………............................................................................................

LIST OF APPENDICES ......................................................................................................... xii

LIST OF ABBREVIATIONS.............................................................................................

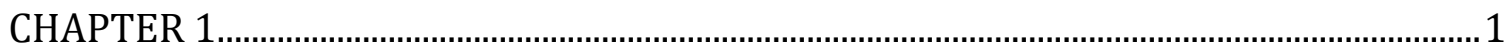

Situating the Research Problem ..............................................................................................

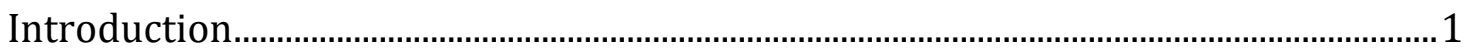

The Un-Official Starting Point: The Innovative Article .................................................... 2

Canada's Multiculturalism: The Ukrainian Input ................................................................ 4

Ukrainians in Canada ........................................................................................................

Personal \& Professional Incentives ......................................................................................6

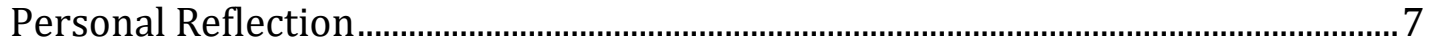

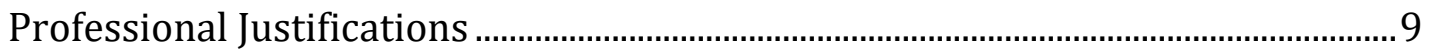

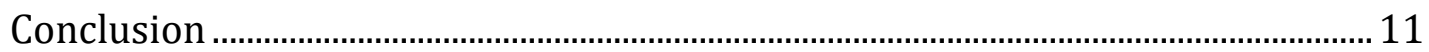

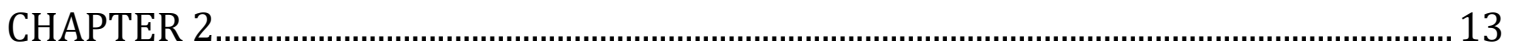

Literature Review \& Synthesis, Study Purpose, Research Question................................. 13

Introduction........................................................................................................................... 13

Literature Search Strategy ………………………………………………………... 13

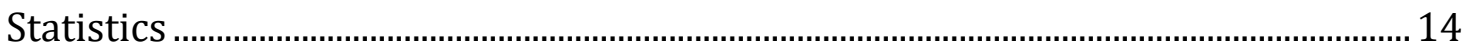

Canada's Seniors: Defined by the Numbers ............................................................... 15

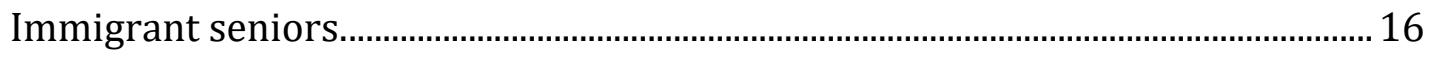

Defining Concepts .................................................................................................... 18

Culture: An Inherent Lens ......................................................................................... 18

Cultural Competence \& Culturally Competent Care: Defined ..................................... 20

Cultural Incompetence \& Health Outcomes................................................................ 21

Model of Cultural Competence ....................................................................................... 24

Process of Cultural Competence in the Delivery of Healthcare Services Model 24

Constructs of Cultural Competence ............................................................................... 26

Critical Appraisal of the PCCDHS Model......................................................................2 29

The Current Landscape of CCC ...................................................................................... 30 
Cultural Competence and Person-Centered Care ........................................................... 31

Healthcare Providers: Personalities \& Biases............................................................. 35

Nurses' perspectives: Caring for culturally diverse persons................................. 37

Policy Makers and Focus on CCC ............................................................................... 40

Immigrant Status: Culture and Health Outcomes ....................................................... 43

Increasing Availability of CCC: Targeting Practice \& Education ..................................... 44

Ethnic Match: Potential for Enhancing CCC ................................................................ 45

Ethnic Match: The Opposing Views .............................................................................. 47

Cared for by Culturally Diverse HCPs: Clients' Perspectives.................................... 50

Nursing Education and Training: Improving Cultural Competence......................... 53

Chapter Summary ………………….................................................................... 55

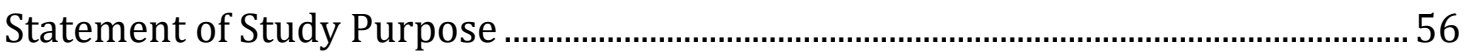

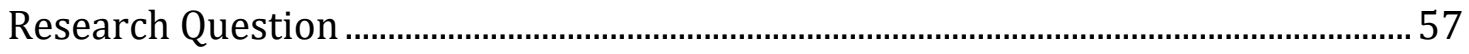

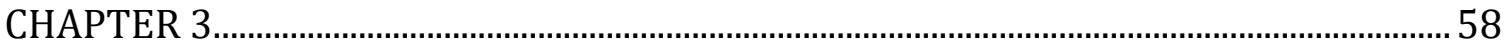

Theoretical Lens, Methodology \& Design …………………………………………….... 58

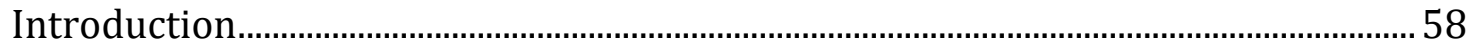

Theoretical Lens: The World Through a Thousand Eyes............................................... 58

Applying Theory and Philosophy to Real Life...........................................................6 61

Methodological Approach: Nurses \& Storytelling ......................................................... 62

The Chosen Methodology .................................................................................................6

Participant Selection .................................................................................................. 64

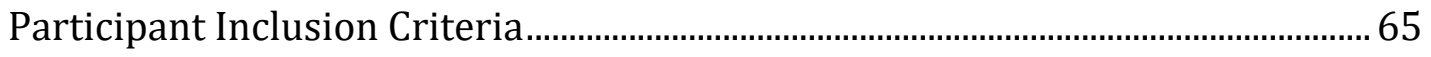

Recruitment Method ..................................................................................................... 66

Understanding the Participants ............................................................................... 68

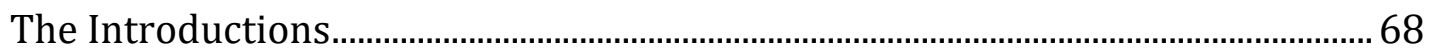

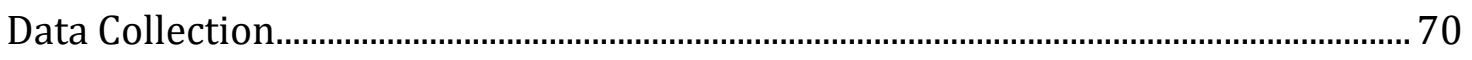

Interviews: Text \& Interpretation .......................................................................... 70

Photography: The Visual Narrative ............................................................................ 72

Protecting the Participants: A Researcher's Duty …………………………………..... 74

Data Analysis: The What \& The How ……………………………………………...... 74

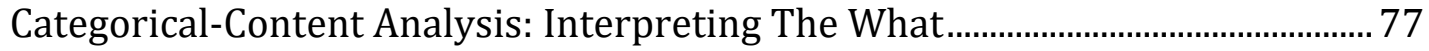

Categorical-Content Analysis Steps .............................................................................. 78

Analyzing the How: Round Hole, Square Peg ........................................................... 80

Imagery \& Research .......................................................................................................... 82 


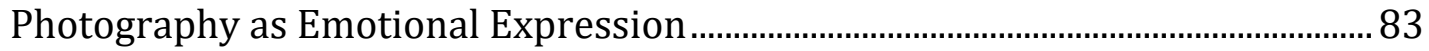

Visual Analysis: Interface of Word and Image. ......................................................... 85

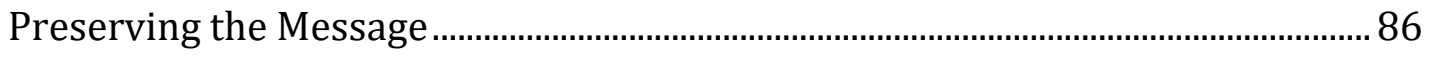

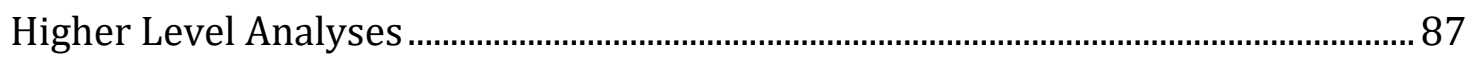

Reflexivity ............................................................................................................. 89

The Metaphorical Analysis: Creating a Graphic …………………………………... 90

Rigor of Narrative Research ........................................................................................ 91

Ambiguity of the Narrative Approach ……………………………………………... 93

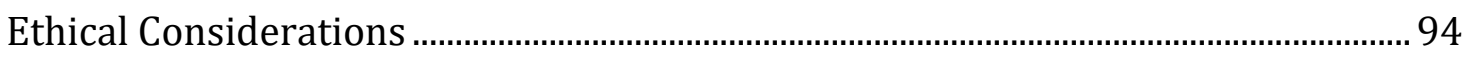

Photography and Ethics ...................................................................................... 96

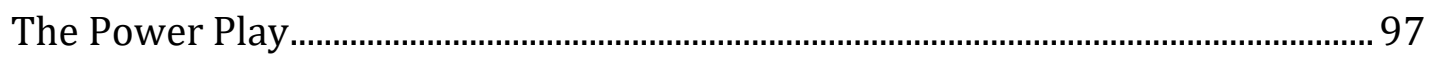

Understanding My Role ........................................................................................... 99

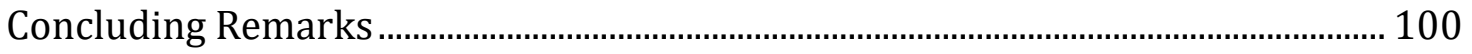

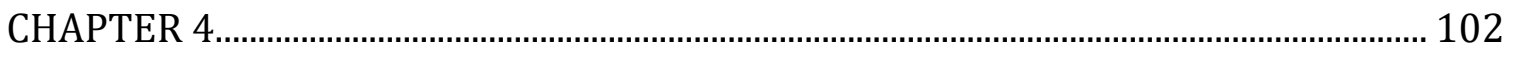

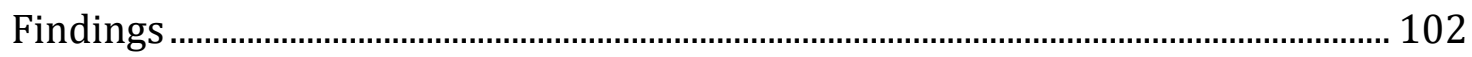

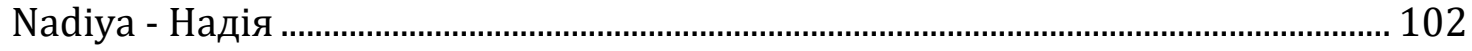

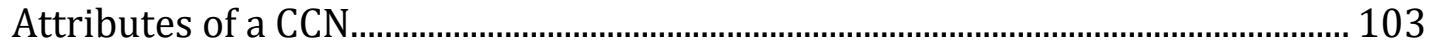

The Nurse's Cultural Wisdom: "I know my people” ................................................ 106

Historical influences: The past must be explored. ................................................. 107

Boundaries of cultural knowledge. ....................................................................... 108

Communication: I speak your language................................................................... 110

Homelike Environment: “A Very Ukrainian Environment” .................................... 112

Pushing Against the Barriers to CCC .......................................................................... 117

Nadiya's Conceptualization of CCC ..................................................................... 120

Zolota Seredunka: CCC as Keeping the Golden Middle ............................................... 120

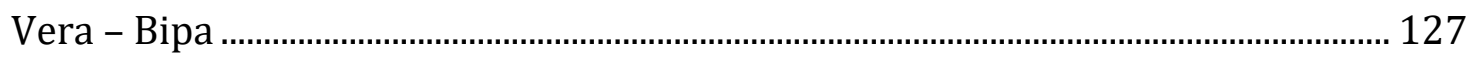

Collegial Support: "We share the care" ................................................................ 127

Client's Needs Through Personalized Lens: Knowing the Expectations ............. 131

The Ukrainian Setting: My House, My Rules.............................................................. 133

A Gardener Tending to the Planter: The CCN........................................................... 135

Vera's Conceptualization of CCC: Supporting the Livinghood ................................... 138

Livinghood: A New Perspective on CCC................................................................... 138

Nadiya and Vera: Different Homes, Distinct Personalities, Echoed Themes......... 141 


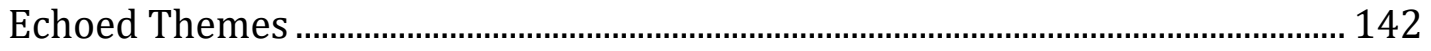

Participants \& Personalities: The Context of the Narrative.................................... 143

The Shadows of Their Personalities............................................................................. 147

Cultural Insightfulness ............................................................................................ 149

Nadiya's experience of cultural insightfulness. ……………………………….... 150

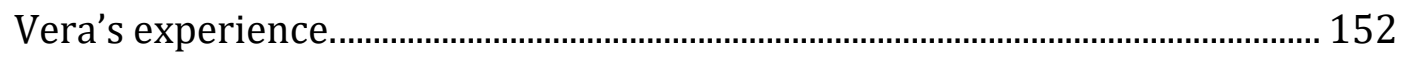

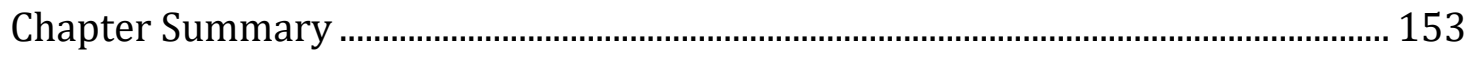

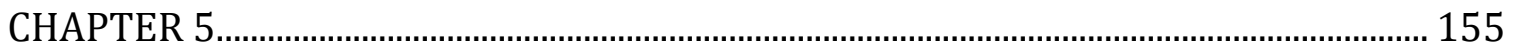

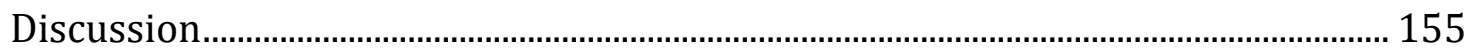

Integrating the Findings: A Visual Depiction ………………………………………... 155

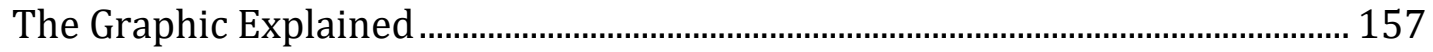

Exploring the Major Themes ..................................................................................... 159

Honoring the Resident .............................................................................................. 159

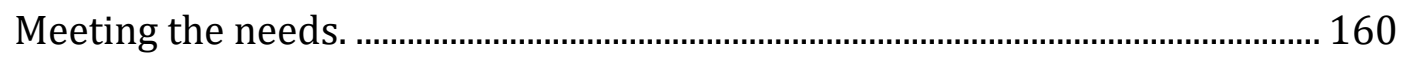

Understanding livinghood. .................................................................................... 163

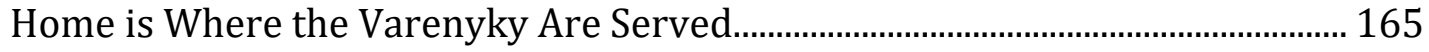

Ukrainian language................................................................................................ 170

Organizational barriers to CCC. ……………………………………………...... 174

The CCN as The Doorway to CCC............................................................................ 177

Applying Campinha-Bacotes' model....................................................................... 180

Cultural Insight as the Solid Foundation.................................................................. 183

Greater than an ethnic match................................................................................ 184

Cultural insight as distinct from cultural intuition. ................................................ 185

I+Thou. .............................................................................................................. 188

Why cultural insight is not ethnocentrism...................................................... 192

The Researcher's Reflection: More Questions Than Answers ..................................... 192

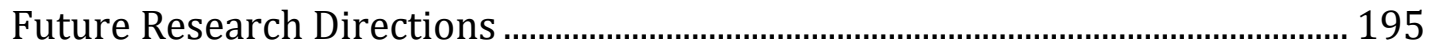

The Outliers .......................................................................................................... 197

Implications for the Healthcare Organizations: Immersed Training........................ 199

Policy Implications: Hiring Practices.......................................................................... 202

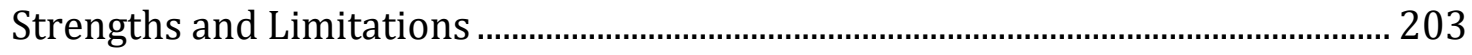

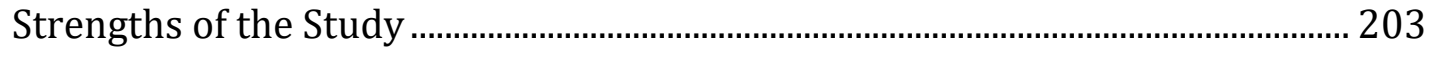

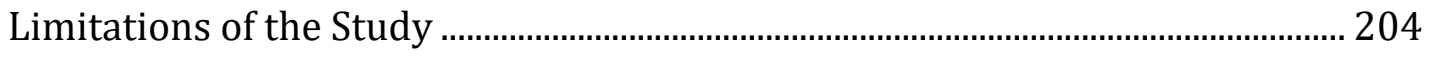




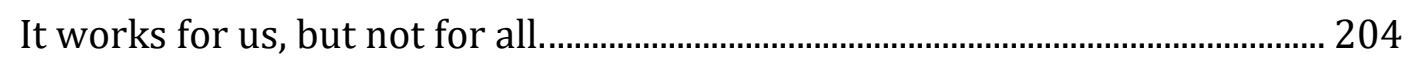

We should have taken 'selfies' first.......................................................................... 205

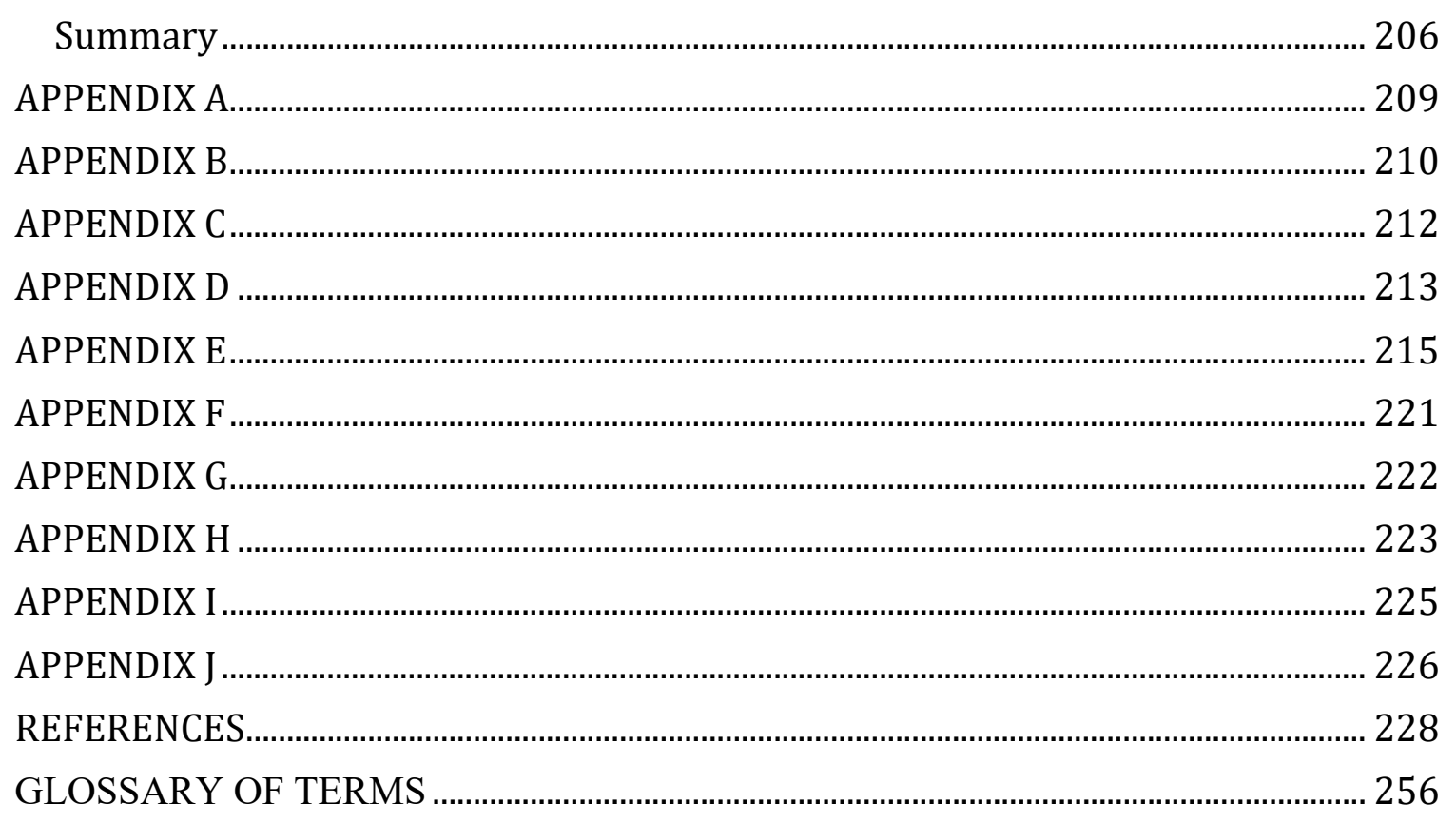




\section{TABLE OF FIGURES}

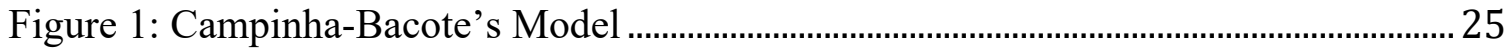

Figure 2: The Ukrainian Lunch......................................................................................... 114

Figure 3: Holubczi ta Vushuvanka ................................................................................... 114

Figure 4: The Kobzar and the Resident ........................................................................ 124

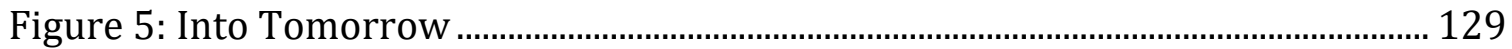

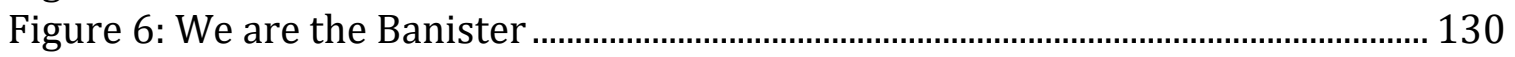

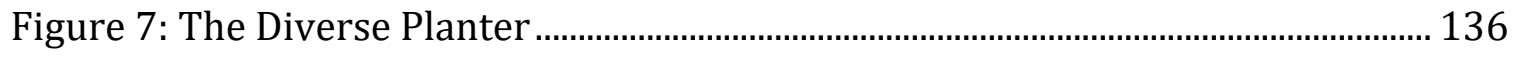

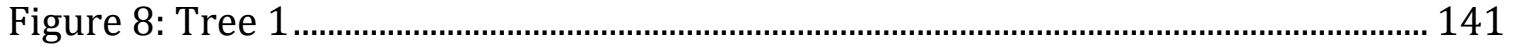

Figure 9: Tree 2 ………………………………………………………………… 141

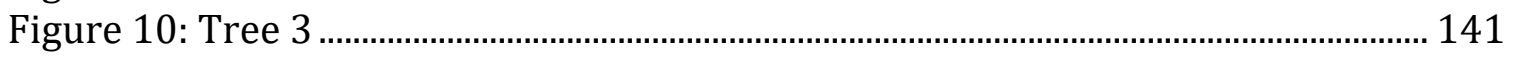

Figure 11: Culturally Component Care in Mono-culture Health Care Facilities ....... 156 


\section{LIST OF APPENDICES}

APPENDIX A Recruitment Poster

APPENDIX B Participant Recruitment Announcement Details

APPENDIX C Third Party Letter

APPENDIX D Interview Guide: First Interview

APPENDIX E Information and Consent Form

APPENDIX F Photography Activity: Information Handout

APPENDIX G Second Interview Guide: Camera Collection

APPENDIX H Interview Guide: Third Interview

APPENDIX I REB Certificate

APPENDIX J Demographic Background and Cultural Sensitivity Survey 


\section{LIST OF ABBREVIATIONS}

CCC - Culturally competent care

GTA - Greater Toronto Area

HCP - Healthcare professionals

LTC - Long-Term care

LTCH - Long-Term Care Home

PCC - Person Centered Care

RN - Registered Nurse

RPN - Registered Practical Nurse 


\section{CHAPTER 1}

\section{Situating the Research Problem \\ Introduction}

Every study must start somewhere. The seed for this particular study had been planted in my head long before I had decided to enroll in graduate studies. First, there was my personal, somewhat rocky journey of immigrating to Canada at a young age that had instilled in me the importance of culture as an element of identity. Then, during my undergraduate studies with the Daphne Cockwell School of Nursing at Ryerson University, I learned that it is important to incorporate my client's cultural needs into my care by observing my preceptors. After graduation, working as a Registered Nurse (RN) in the real world gave me the opportunity to practice the skills of cultural competence so that I was now able to demonstrate respect for the diversity of my clients.

Then, in 2011, I came across an innovative article by Stuart and Nielsen (2011) about the unique understanding nurses have of the needs of clients with whom they share a cultural background. Reflecting on my own experience as a nurse, I noted the ease with which I was able to care for clients who were Ukrainian, like me. I would speak to them in our shared language, effortlessly understanding the nuances of their verbal and nonverbal communication. When I found myself caring for clients of diverse cultures, it was often a challenge to establish professional camaraderie and understanding as part of the therapeutic relationship. Putting together the puzzle pieces, the idea for this study was born. It was my intent to gain a deeper understanding of how Ukrainian nurses conceptualize and provide culturally competent care, or CCC, to the older Ukrainian residents of Ukrainian-specific long-term care homes (LTCHs) in the Greater Toronto Area (GTA). 
This introductory chapter further details the personal, professional and scholarly explanations for the study presented in this written work. It marries the history of the older Ukrainian immigrants in Canada with the contemporary focus of Western healthcare on culture, bridging the two through the concept of CCC, which is the main interest of this study. The reader is given insight into the statistical information associated with Ukrainian immigrants in Canada, as well as a concise overview of the current perspectives on CCC as the scholarly justifications for this study.

\section{The Un-Official Starting Point: The Innovative Article}

In 2011, nursing scholars Lynne Stuart and Anne-Maree Nielsen published an article in the journal Contemporary Nurse detailing their perspectives on what constitutes CCC for Aboriginal people of Australia. The article title read: Two Aboriginal Registered Nurses Show Us Why Black Nurses Caring for Black Patients is Good Medicine. This was my first introduction to the possibility that a different, perhaps even better, quality of care can be provided by nurses who share a cultural background with their clients. The ideas in this article arise out of Stuart and Nielsen's independent graduate work around health and cultural challenges of the Indigenous population of Australia, and are further supported by their personal experiences as professional Aboriginal RNs. The authors' belief that I can provide "good medicine" to Ukrainian immigrants in the context of the Canadian healthcare system was intriguing and made me wonder if this notion holds across other cultures, including among Ukrainian immigrants in Canada.

At the start of the article, the authors immediately acknowledged that their cultural background as Aboriginal RNs predisposed them to provide better quality of CCC to other Aboriginal people. They explained that the poor health statistics of the 
Indigenous people in Australia are the result of the inability of dominant Western healthcare practices to address the cultural needs of this group. On a personal level, the inability of non-Aboriginal healthcare professionals (HCPs) to understand the cultural needs of the Aboriginal clients is also attributed to the difference in cultural heritage by the authors. Stuart and Nielsen (2011) explain that "although non-Aboriginal health professions are well intentioned, their birth excludes them from cultural healing knowledge and is a far cry from the good medicine that can emanate from the hands of Aboriginal nurses toward their people" (p. 98). To reinforce these ideas the authors describe their understanding of the colonization experiences of Indigenous people, linking it to the health crisis afflicting the cultural group. They understand the essence of what it means to be Aboriginal.

Care that is provided to Aboriginals by Aboriginal nurses, conclude Stuart and Nielsen (2011), is derived from cultural groundings that are linked to a shared ancestry. This unique connection between the care recipients and care providers arms the latter with an introspective understanding of the needs of their clients. Sharing the cultural background with the client, explain the authors, means having a vested interest in the health of this individual.

Stuart and Nielsen (2011) go on to discuss the specifics of increasing the proportion of Aboriginal nurses in Australia's healthcare workforce. My interest, however, had lingered over the notion that nurses may be able to provide more fitting care to clients with whom they share a cultural background. To gain better understanding of this concept through scholarly research I deduced that culturally equivalent clientprovider dyads are most likely to be found in culture-specific LTCHs. As I began to 
develop the study protocol as part of the Master of Nursing thesis, I chose to focus on exploring the experiences of Ukrainian nurses of providing CCC to older Ukrainian immigrants residing in Ukrainian-specific LTCHs. The upcoming section situates Ukrainian immigrants as a vital part of Canada's social fabric, which reinforces the importance of studying the experiences of this segment of the country's population within the healthcare sector.

\section{Canada's Multiculturalism: The Ukrainian Input}

In 1971, the government of Canada instituted an official multiculturalism policy, the first of its kind worldwide (Government of Canada, 2012). Aptly titled the Multiculturalism Policy of Canada it focused on promoting a sense of belonging and cross-cultural understanding among the citizens of the country. Canada's 'father of multiculturalism' was a first-generation Ukrainian-Canadian Paul Yuzuk, who was appointed to the Canadian Senate in 1963 (Government of Canada, 2009). During his inaugural speech Senator Yuzuk defined Canada as a multicultural nation, recognizing the presence and value of diverse ethnic and cultural groups within Canada's social milieu. Soon after, in 1982, the Canadian Charter of Rights and Freedoms was introduced promoting equality among Canadians of all religious, racial, cultural or ethnic affiliations (Government of Canada, 2015). Multiculturalism had, thus, become a fundamental canon of Canada, and continues to shape the country's citizenship today.

\section{Ukrainians in Canada}

Senator Yuzuk's parents were among the pioneer Ukrainian settlers in Canada (Manitoba Historical Society, 2015). Ukrainians, as a cultural group, have been acknowledged for the significant contribution they have made to Canada's heritage 
(Couton, 2014). Between 1891 and 1914, the majority of Ukrainians coming to Canada known as persha hvulya, or the first wave - would settle in the West; today, as Canada welcomes the fourth wave of immigrants from Ukraine (starting in 1991 and onwards), the largest Ukrainian population continues to live in Manitoba and Saskatchewan (Toronto Ukrainian Genealogy Group, 2005). As a nation, Canada is home to the third largest settlement of Ukrainians in the world. According to the 2011 Canadian Census, over 1.2 million individuals of Ukrainian heritage reside in Canada - a number exceeded only by the settlements in Russia and Ukraine itself.

The province of Ontario houses the largest proportion of Canada's population, with over thirteen million residents being registered in June 2014 (Ontario Ministry of Finance, June 2015). Ontario, according to the Ukrainian Canadian Congress (2012) is home to a community of 330000 Canadians of Ukrainian background. The Ukrainian presence is especially well established in Toronto, Ontario, which has been my home since I immigrated to Canada in 1999. The city boasts a large number of Ukrainian churches, schools, daycare centers, professional/business clubs, community associations, stores, dance ensembles, credit unions, and varied social services organizations. According to Couton (2014), the Ukrainian community "has one of the strongest networks of ethnospecific organizations in Canada" (p. 14). The list of Ukrainian-specific organizations is extensive; however, of particular interest to the present study, as was explained earlier, are the Ukrainian-specific LTCHs in the GTA.

Although older adults of Ukrainians heritage, the majority of whom are immigrants to Canada, live at a number of LTCHs throughout the city that have larger clusters of Ukrainian residents, there are four well-established not-for-profit Ukrainian- 
specific LTCHs in the area. A look through the websites of these organizations reveals deep commitment to serving the Ukrainian community and honoring the Ukrainian culture, as expressed in their care philosophies and organizational values. As these facilities are nestled amid a bustling multicultural metropolis, one is left to ponder how does the frontline staff of these mono-culture LTCHs provide CCC to the residents? This question has, therefore, shaped the direction of the thesis for my Master of Nursing studies. In the pages that follow I detail my own connection to the Ukrainian community at large and further reveal the rationale of this study that aimed to narrate the experiences of frontline staff of Ukrainian-specific LTCHs with providing CCC to older Ukrainian immigrant residents.

\section{Personal \& Professional Incentives}

The literature on CCC provides academic justification for the study based on facts, scientific explorations and expert knowledge. This study, as will be detailed later, adopts a qualitative perspective to research. As part of the qualitative approach, the primary investigator is called to share with the reader her personal experiences, values, thoughts and beliefs that have given support to the development of the study (Ortlipp, 2008). Such conscious reflective practice promotes transparency of the research process while giving context to the study from the researcher's personal perspective (Ortlipp, 2008). To build on the scholarly incentives for the study detailed herein, I present the reflections emerging from my own personal background as an immigrant from Ukraine and the professional experiences as a Canadian-educated nurse. 


\section{Personal Reflection}

I am an immigrant. Regardless of how many years I live in Canada, what level of education I attain or the cultural diversity of my social circle, I will continue to identify myself as an immigrant from Ukraine. I have been lucky to be a member of, what the older immigrants have labeled as the fourth, and most recent, wave of immigrants, the ones who left Ukraine after the collapse of the Soviet Rule. From the outside, I appear to be well acculturated to the Canadian way of life. I follow the current fashion scene, work in an English-speaking environment, and can even name all of Canada's provinces and territories. But at home, I have traditional embroidered blouses hanging in my closet that I wear during Easter and Christmas holidays in accordance with the Julian calendar, my most prized possession being a blouse done in the Borschivski style as it reflects my father's heritage. At mealtimes, I ask for more cheese curds on my poutine and put maple syrup on my pancakes, but I always leave room for some of my mother's homemade borsch, or beet soup. I am fluent in Ukrainian and have read classical literature written in my native language, learning by heart the poems of Taras Shevchenko, a Ukrainian poet. But I was not always so comfortable with this intercultural way of being.

During the first few years of settlement in Canada, I shunned away from my Ukrainian background. I wanted, so desperately, to fit in with my peers who spoke of North American pop stars; instead, I had an autographed poster of Ukraine's famous singer who was older than my dad! It was as I began to mature in later years of secondary education that I began to appreciate the uniqueness of my cultural background. As I was being exposed to studies of sociology, languages and history, I began to reflect on my experience as an immigrant in creative writing courses. Today, I have found a way to preserve my cultural identity by adhering to traditions that have been passed down to me 
through generations. I cook ethnic foods, celebrate traditional holidays, attend social events and spend time with Ukrainian-speaking friends. I do, however, continue to look for ways to adjust the traditional delivery of the cultural practices and marry them with the Western ways that I have come to be comfortable with. For example, our family's recipe for borsch sometimes includes a handful of cilantro instead of the parsley that my grandmother would use back home.

I am an only child, and as such, I have begun to take on more responsibility for my parents' well-being, although they are, by societal standards, still young, even at fifty years of age. While, in my eyes, they are the epitome of youth and eternal life, I have heard the conversations among their group of friends of aging and retirement. At the time when my parents and their peers had left the home country some 15 to 20 years ago, nursing homes, like those found in North America, did not exist (and are, to this day, few and sparse in existence). It is not that there were no seniors in the country. Rather, it was the cultural norm for younger family members to care for the old and the sick. It is, therefore, a strange reality for my parents to even consider what nursing home they would choose to live in, if ever necessary. They do, however, acknowledge that this is the reality of life in Canada.

There is an overwhelming preference among my parents' friends to relocate to Florida upon retirement, although this pipe dream is always quickly dismissed when they calculate the physical distance this move would put between them and their children. Local, Ukrainian-specific nursing homes then take the lead in their pseudo-decision making, followed by a lively discussion of the perks of some institutions over others. Among the considerations are the language spoken by staff, the cultural atmosphere and, 
for the money-savvy, the cost of living. I quietly listen to their musings, obediently nodding in agreement when they ask if I will come to visit. In my heart, however, I still feel that cultural pull that steadies in me the conviction that I will do my absolute best to ensure that my parents enjoy their retirement in their own home. Somehow, it just does not feel right to even consider the prospect of long-term care living for my aging parents. It is not part of my culture to do so.

But...just like my parents' generation, now that I am older, more mature, I also wonder about the future. What will happen as I grow old? Will I spend my reversed youth in a long-term care facility? And when I find myself living in a Ukrainian longterm care home, will I be greeted with the comfortable Dobroho Ranku, or a simple "Good Morning"? Will I get to observe religious holidays as I had done for so many years out of my own volition, or will I have to settle for the turkey dinner on December $25^{\text {th }}$ ? What will it be like to be a Ukrainian older adult living in Canada when I reach that age? These musings are the driving force behind my interest in researching the nature of culturally competent care at Ukrainian-specific long-term care facilities. I hope that the findings may help better understand the concept of culturally competent care as it relates to Canada's Ukrainian population. This knowledge, in turn, may be used to direct staff training initiatives to promote continuity of cultural lifestyle for long-term care residents today and in the years to come.

\section{Professional Justifications}

Canada's population is becoming increasingly diverse in culture, and is aging (Schim, Doorenbos, \& Borse, 2005). The heterogeneity of Canada's ageing population, as emphasized by Ujimoto (1994), means cultural and ethnic differences warrant 
attention in the development and implementation of health care initiatives aimed at this subgroup. Ujimoto emphasizes that, "ethnic and cultural differences in perceptions of health and illness, responses to illness, health status, utilization of services, and coping responses vary considerably, thus rendering a standard regimen inappropriate or ineffective" (p. 202). When the healthcare services are structured according to the dominant culture, they may ignore the different needs of the culturally diverse clients. This narrow approach to care places certain groups, such as older immigrant adults, at risk of receiving poor healthcare. Mullings and Gien (2013) point out that immigrant seniors, when compared to their non-immigrant peers, are more likely to have restricted access to healthcare and experience poor health outcomes. Given that the proportion of older immigrants is expected to rise exponentially in the next twenty years, the importance of addressing the health needs of this group is further emphasized (Employment and Social Development Canada, 2015).

Increasing the availability of $\mathrm{CCC}$ has been proposed as a reliable approach for improving the health outcomes of older immigrant adults since this approach to care is connotative with enhanced communication between the care recipients and providers, improved adherence to treatment and overall reduction of other related barriers to healthcare access (Schim et al., 2005). There is, however, a persistent lack of skills and knowledge related to CCC among the healthcare professionals (HCPs), including nurses in Ontario (Schim et al., 2005). In order to propose a solution for enhancing the quality and availability of CCC to older immigrant adults, it is first important to establish an understanding on how this type of care is defined and provided by the frontline healthcare professionals. Commonly, research on $\mathrm{CCC}$ with respect to healthcare has been restricted 
to effectiveness of educational programs at enhancing cultural knowledge (Cooper Brathwaite \& Majumdar, 2005) from the perspective of nursing students (Brenna \& Cotter, 2008; Lipson \& DeSantis, 2007; Long, 2012; Sumpter \& Carthon, 2011). Furthermore, studies on cultural competence of HCPs have been limited in settings, focusing primarily on acute hospitals (Capell, Dean, \& Veenstra, 2008; Schim et al., 2005), home care (DiCiccio \& Bloom, 2003), and community and public health (Bernal \& From, 1993). Additionally, the focus of these studies has been narrow in its cultural breadth, primarily examining the experiences and outcomes with CCC of cultural minority groups (Durst, 2005).

Referring back to Stuart and Nielsen's (2011) article, there is some support in the literature for my notion that nurses are able to provide better quality of care to clients with whom they share a cultural background. At press time, however, no research focusing on CCC and the Ukrainian-specific LTCHs in the GTA was identified during the literature review. Carrying out research with Ukrainian nurses caring for older Ukrainian immigrants residing in Ukrainian-specific long-term care environments will be instrumental in gathering information tailored to the needs of this population. Studying the phenomenon of CCC from a qualitative perspective is essential for amending the quantitative findings and giving them context, as well as for advancing best practice guidelines (Allen et al., 2008).

\section{Conclusion}

Given the current demographic shift in Canada, both with respect to ethnic diversity and ageing, it has become vital to nurses, whose professional obligation is to provide individualized care, to include cultural competence in their professional growth 
and development. This topic is of particular interest to me because of my professional background as a nurse and cultural background as a Ukrainian immigrant, and thus this study focused on exploring Ukrainian nurses' perceptions of providing CCC to older Ukrainian adults living in Ukrainian-specific LTCHs. 


\section{CHAPTER 2}

\section{Literature Review \& Synthesis, Study Purpose, Research Question Introduction}

This chapter presents a review of the current state of literature on CCC in healthcare. To guide the reader's understanding, definitions of the related concepts of culture, cultural competence and culturally competent care are presented. Exploring the experiences and perceptions of both older immigrants and the HCPs through literature review provided an overview of how $\mathrm{CCC}$ is received and delivered. Additionally, the chapter explores the approaches for addressing lack of CCC in healthcare, among them the practice of ethnic match and integration of CCC content into the education and training programs for HCPs. It is my aim to present here the rationale for engaging in research that will narrate the experiences of frontline nurses working in mono-culture LTCHs of providing CCC to their older immigrant residents.

\section{Literature Search Strategy}

The literature included in this review was retrieved from electronic databases, among them CINAHL, ProQuest Nursing Journals, PsychINFO, Social Services Abstracts, Psychology and Behavioral Sciences Collection and Medline (OVID). The key search terms used alone and in combination included culture, cultural competence, culturally competent care, cultural sensitivity, cultural awareness, person centered care, older immigrant, elderly, long-term care, nursing homes, Ukrainians, healthcare professionals, nurses, and minority residents. In reviewing the abstracts and titles of the retrieved articles, I rejected those that did not identify culture, cultural competence or culturally competent care as the primary area of focus. Further to this, manual review of the reference lists of highly relevant articles was carried out with the intent of identifying 
related literature. Although the electronic data base search was only restricted to publications in English, no limitations were placed on the years of publication to allow the writer to develop a sense of the history of the development of CCC in healthcare. As the result of the search, 78 articles published between the years 1979 and 2015 were identified and included in the review for this study, among them integrated literature reviews, concept analysis papers, as well as peer-reviewed qualitative, quantitative and mixed methods studies. Additionally, statistical information and reports were collected through publications from the government and professional organizations. Three textbooks were also reviewed as well as one doctoral dissertation. In addition to information focusing on nursing and healthcare, literature from the related fields of philosophy, psychology and sociology was referenced to further the understanding of theoretical scholarship related to CCC.

\section{Statistics}

In the previous chapter statistical information regarding the Ukrainian population in Canada was presented to emphasize the social and historical contributions this cultural group has made to the Canadian society. Research has demonstrated that cultural influences often persist through multiple generations (Falicov, 2005), therefore, it is logical to conclude that the Ukrainian culture brought into Canada by the first wave of immigrants from Ukraine will continue to shape the values and cultural expectations of Ukrainian-Canadians of current and future generations. Falicov (2005) acknowledges that healthcare settings often function as the interface between traditional cultural expectations of immigrant groups and the standards of the dominant Western culture. Acknowledging the extent of integration of Ukrainian immigrants into the Canadian 
context, therefore, emphasizes the need to understand how CCC offered to this group is conceptualized and provided by frontline HCPs. This section further details statistical demographic information about the older adult population in Canada, including a focus on senior immigrants. This information is intended to assist the reader to understand the role that CCC has in the lives of older immigrants.

\section{Canada's Seniors: Defined by the Numbers}

Seniors, according to Statistics Canada (2013), are individuals 65 years of age and older. In 2011, there were 5 million seniors living in Canada, a number that is expected to double within the next twenty-five years (Human Resources and Skills Development Canada, 2013). Seniors are the fastest growing age group in Canada; the country's proportion of seniors has been growing exponentially as a result of increased life expectancy, ageing of baby boomers and lower fertility rates (Statistics Canada, 2014). As recently as July $1^{\text {st }}, 2014$, close to $16 \%$ of Canada's population was age 65 years and older (Statistics Canada, 2014). It is projected that, in 2036, seniors will make up 23\% of population in the province of Ontario; meanwhile, in the year 2011, this number was only $14.2 \%$ (Employment and Social Development Canada, 2015). It is anticipated that the demands placed on the healthcare system by this segment of the population will continue to rise along with the increase in population size. By and large, Canada's population is growing older; therefore, the needs of seniors are becoming a national priority (Romanow, 2002).

Statistics Canada (2013) makes note of the living arrangements of Canada's seniors. Living as part of a couple continues to be the most common living arrangement for seniors, but the number of seniors living in special care facilities - collective 
dwellings that offer living assistance and professional healthcare - increases with age (Statistics Canada, 2013). Of the 5 million seniors living in Canada in 2011, approximately 7\% had been living in special care facilities (Statistics Canada, 2013). Although only $1 \%$ of those seniors had belonged to the age category ranging between 65 and 69 years, this number was closer to $30 \%$ for seniors ages 85 and over (Statistics Canada, 2013). As Canadians grow older, more and more are making LTC facilities their permanent homes. Collectively, the growing size of the older adult population, along with the increased tendency to relocate to LTCHs in advanced age, emphasize the importance of understanding how to care for culturally diverse older immigrant groups in the context of LTC settings.

Immigrant seniors. A significant proportion of Canada's seniors are immigrants. Statistics Canada (2010) defines immigrants as persons who, although they are currently residing in Canada, were actually born outside of the country. According to a Working Paper Series on immigrant seniors in Canada compiled by Ng, Lai, Rudner, and Orpana (2012), 30\% of Canada's seniors in 2006 were immigrants. This segment of the seniors' population is rather diverse itself, differing both in length of settlement in Canada and cultural background. The majority of Canada's immigrant seniors identified in the 2006 Census had actually migrated to Canada prior to 1976 , while only $6 \%$ were classified as recent immigrants, having settled in Canada between 1996 and 2006 (Ng et al., 2012). The proportion of senior immigrants in Canada continues to grow annually, even if at lower rates. The more recent senior immigrants are more likely to settle in one of Canada's three large metropolitan cities, including Toronto, Montreal and Vancouver (Ng 
et al., 2012). Toronto attracts the highest proportion of recent senior immigrants; in 2006, $67 \%$ of Toronto's seniors were immigrants, report $\mathrm{Ng}$ and colleagues.

The cultural background composition of Canada's immigrant seniors has changed in recent decades, as the proportion of visible minority immigrants is on the rise. While the majority of immigrants settling in Canada prior to 1976 were from Western and Eastern Europe, the decade prior to the 2006 Census saw an increase in immigrants from East and South Asia (Ng et al., 2012). Between 2006 and 2011, the largest proportion of immigrants to Canada was from Asia (Statistics Canada, 2014). Ukrainians were a significant segment of Canada's early immigrants from Eastern Europe, settling in the prairies as early as 1891 , and they continue to emigrate from Ukraine in significant numbers; 1991 marked the start of the fourth wave of Ukrainian immigrants to Canada (Toronto Ukrainian Genealogy Group, 2005). Presently, however, individuals hailing from Europe make up the second largest group of immigrants coming to Canada, preceded only by representatives of visible minorities (Statistics Canada, 2014).

Although the cultural and ethnic composition of Canada's senior immigrant population continues to shift, the large and growing proportion of the country's immigrant seniors attracts the attention of researchers looking to enhance the nation's well-being. Great emphasis in research is placed on understanding the experience of immigrant groups of all ages with the healthcare sector, including seniors since healthcare services are frequently among the first encounters with Canada's social services that immigrants have (Romanow, 2002). Enhancing the availability of quality care for this group is associated with improved health outcomes (Majumdar, Browne, Roberts \& Carpio, 2004). One of the features differentiating immigrants from the 
residents of the host country is their culture. Cultural values brought from their country of origin have been shown to carry over into the lives of immigrants despite settlement in the host country. These earlier values continue to shape their identities and life experiences, including their health (Lieber, Chin, Nihira, \& Mink, 2001).

\section{Defining Concepts}

\section{Culture: An Inherent Lens}

Before further exploration of the literature on $\mathrm{CCC}$, it is important to define culture and its role in healthcare. The College of Nurses of Ontario [CNO] (2009) asserts that everyone has a culture, although, as a concept, it is not always simple to define. When viewed in basic terms, culture is understood as the interplay of ethnicity, language and religion; furthermore, it is commonly defined with respect to a group (Maddalena, 2009). Among the first nursing scholars to consider the link between culture and healthcare was Dr. Madeleine Leininger (1991), who developed the Transcultural Theory of Nursing acknowledging that cultural similarities and differences exist among human groups. Leininger (1991) defined culture as "the learned and transmitted values, beliefs, and practices that provide a critical means to establish culture care patterns for the people" (p. 36). Maddelena (2009) simplifies this statement, explaining that culture is like an "inherent lens" or a way of looking at the world and understanding one's own place in it. Culture, therefore, helps people make sense of everything around them and it is suggested that it has elements one is "born knowing" rather than acquired.

Leininger's definition of culture acknowledges the ability of learned and transmitted values to shape people's attitudes and create special meanings unique to a group. This notion is echoed by the Registered Nurses Association of Ontario [RNAO] 
(2007). As one examines the literature on culture, it becomes evident that culture is bound by common experiences, and is transmitted through membership within a specific group (Maddalena, 2009); there is, however, potential for as many variations in the expression of cultural attributes within a single cultural group as there is between distinct cultural groups (RNAO, 2007; Schim, Doorenbos, Benkert, \& Miller, 2007). Leininger (1991) acknowledges that culture is passed down through generations via formal and informal life experiences; although it holds the capacity to adapt according to the group's needs, cultural practices commonly maintain a degree of their integrity and are thus sustained over time. Culture, therefore, while responsive to historical, social and interpersonal factors, is, nevertheless, a relatively stable concept. As such, culture influences how people behave (Maddalena, 2009).

Culture, with its inherent focus on shared beliefs, ideas and practices, shapes people's definitions of health and illness (Capell, Veenstra, \& Dean, 2007), and informs health-related behaviors (Maddalena, 2009; Purnell, 2002). Leininger asserted that care provided by nurses should align well with the client's cultural beliefs, practices and values; such an approach is then said to be exemplifying culturally competent care (Murphy, 2006). Given the highly varied and individualized expressions of culture, however, nurses must be cautious of delivering care to their clients based on preconceived notions associated with the client's cultural profile. There is a tendency among humans to be culture bound, meaning people erroneously assume that everyone belonging to a specific cultural group is alike in their values and beliefs (Sue \& Sue, 2003). Such generalizations are at the root of stereotypical attitudes and ethnocentric views, and greatly restrict HCPs' ability to develop cultural competence (Taylor, 2005). 
While it is unrealistic to expect every HCP to learn the intricacies of every culture in existence, Van-Ryn and Burke (2000) warn that gaining only superficial knowledge of the primary beliefs and values associated with individual cultural groups may create discriminatory stereotyping, and subsequently lead to poor health outcomes for the care recipients. Consequently, in order to effectively manage individuals' well-being, HCPs are called to develop extensive understanding of their cultural expectations (Capell et al., 2007). The phenomenon of addressing clients' culturally mitigated health needs is termed cultural competence and falls outside of the spectrum of interpersonal and technical skills that are routinely taught to HCPs (Capell et al., 2007). It is more commonly conceptualized as a process of development through experience, rather than a set of skills learned from a textbook.

\section{Cultural Competence \& Culturally Competent Care: Defined}

In recent years, the significance of cultural competence in Canada's healthcare sector has grown significantly as the result of the country's growing and diversifying population described earlier. Cultural competence is defined in the literature as a "set of congruent behaviors, attitudes and policies that come together in a system, agency or among professionals and enable that system, agency or those professionals to work effectively in cross-cultural situations" (Renzaho et al., 2013, p. 262). Many recognize cultural competence as the professional and moral duty of HCPs and healthcare organizations (CNO, 2009; Sanchez, 2008). HCPs who demonstrate respect for and sensitivity to clients' value-laden preferences, and appropriately incorporate cultural elements into the care routine, are said to be delivering culturally competent care, or CCC (DiCicco-Bloom \& Cohen, 2003). CCC therefore is the ability to adapt approaches 
to care in a manner that is respectful of the client's cultural context (El-Amouri \& O’Neill, 2011; Mattson, 2000). Since CCC is defined in terms of 'ability', it is evident that the availability of CCC depends on the capacity of HCPs to readily integrate the skills and attitudes with knowledge and understanding of the client's culture so that the needs of that individual are appropriately met.

Delivering CCC is not an easy task. Cultural competence is difficult to attain, as it cannot be succinctly taught in a classroom environment akin to technical skills, such as wound care (Dudas, 2012). Rather, building cultural competence is a gradual and iterative process that includes complex, ongoing and dynamic interactions between the nurse, culturally-diverse clients and the surrounding world; overall, cultural competence is subject to change and growth with time and experience in clinical practice (Schim et al., 2007; Tabari-Khomeiran, Kiger, Parsa-Yekta, \& Ahmadi, 2007). The delivery of $\mathrm{CCC}$, however, is an expectation of best practice delivery, and it is inferred in the literature that exemplary care cannot exist without some aspect of culture purposefully and consciously addressed by the nurse (CNO, 2009).

\section{Cultural Incompetence \& Health Outcomes}

Given that cultural competence is a progressive and evolving process, logic would dictate that there exists the opposing state of cultural incompetence, a point at which care is completely devoid of cultural sensitivity. The literature conceptualizes this state as the initial stages of development of cultural competence. Cross, Bazron, Dennis, and Isaacs (1989), for example, described the development of cultural competence as progressing along a continuum of six stages; the degree of cultural incompetence speculated above corresponds to the first three stages of the continuum. The first stage, termed cultural 
destructiveness, is a point at which effort is intentionally directed at destroying a culture and inflicting damage on those associated with it (Cross et al., 1989). The malicious intent is reflective of incompetence.

At the second stage of the continuum, cultural incapacity, healthcare organizations in general, and HCPs specifically, lack the ability to provide $\mathrm{CCC}$ to clients representing diverse cultural groups (Cross et al., 1989). While this incapacity is not necessarily rooted in the intention to cause harm as it is with cultural destructiveness, it does arise out of overwhelming conviction of the superiority of one's own culture, a belief recognized in the literature as ethnocentrism or bias (Cross et al., 1989). The individual demonstrates no awareness or understanding of other cultures, and is, therefore, likely incapable of providing CCC. Taylor (2005) warns that individual HCPs may be well-intentioned, but when they fail to modify their care approaches to meet the cultural needs of their clients, they rely on a one-size-fits-all approach that breeds professional incompetence and disregard of the clients' preferences. At the organizational level, cultural incapacity is reflected in policies, rules and guidelines that promote discrimination. At the third stage of cultural blindness, all people are treated the same, which is discriminatory in its own way, as this approach disregards the unique features of the various cultural groups, explain Cross and colleagues.

The remaining stages following cultural blindness, in order, are cultural precompetence, cultural competence and cultural proficiency (Cross et al., 1989). The outlook on cultural competence improves in these final three stages identified by Cross and colleagues. Organizations, for example, that have achieved the level of cultural proficiency, the highest degree of cultural competence attainment, demonstrate respect 
and advocate for culture, engage in research to advance CCC, and have staff well-trained in cultural competence (Cross et al., 1989). It should be, therefore, the aim of all representatives of healthcare to undertake the development of cultural competence with the aim of practicing at the level of cultural proficiency.

In today's culturally diverse society, culturally incompetent care, born out of ignorance and stereotypes, is not an acceptable mode of care (Briggs, Briggs, Miller \& Paulson, 2011; Van-Ryn \& Burke, 2000). Yet, research does acknowledge the persistent lack of cultural competence in healthcare; this insufficiency is associated with adverse health outcomes among older immigrant adults and ethnic minorities, groups disadvantaged due to their socio-cultural, financial and immigrant statuses, which, collectively, stem from structural factors (Taylor, 2005). More specifically, the health disparities that persist among these disadvantaged groups are associated with providerclient miscommunication due to language disparity, limited involvement of clients in the health-related decision-making process, clients' distrust of HCPs, and the ineffective interpersonal styles of HCPs (Betancourt, Green, Carrillo, \& Ananeh-Firepong II, 2003; Cricco-Lizza, 2006; Kaplan, Calman, Golub, Davis, Ruddock, \& Billings, 2006; Kennedy, Mathis, \& Wood, 2007; Perloff, Bonder, Ray, Ray, \& Siminoff, 2006; Saha, Beach, \& Cooper, 2008). All of these factors persist in concert with the lack of CCC. The list of potential adverse outcomes associated with healthcare's cultural incompetence is extensive and worrying.

It is through enhanced availability of CCC, however, that the adverse healthcare occurrences experienced by culturally diverse older immigrant adults may be mitigated since CCC is associated with improved communication, strengthened relationships with 
HCPs and overall reduced healthcare disparities (Betancourt, Green, Carillo, \& Park, 2005; Dudas, 2012; Like, 2011). Healthcare organizations and the care providers themselves benefit from CCC alongside the care recipients (Taylor \& Alfred, 2010). For instance, organizational advantages associated with CCC include enhanced healthcare spending and decreased malpractice claims, although benefits such as these may of greater significance in countries with no universal healthcare (Betancourt et al., 2005; Dudas, 2012).

\section{Model of Cultural Competence}

The growing interest in CCC within healthcare settings, and in particular among nurses, dates back to Leininger's (1991) pioneering work on transcultural nursing. To reduce the ambiguity of describing cultural competence, scholars have further revised the phenomenon of cultural competence based on its conceptual and operational definitions and condensed these into models of care. Such models are frequently adopted into practice by individual HCPs or by organizations as a whole. A description of a model by Campinha-Bacote (2002), titled the Process of Cultural Competence in the Delivery of Healthcare Services (PCCDHS model) is presented here as a way to deepen the understanding of CCC.

\section{Process of Cultural Competence in the Delivery of Healthcare Services Model}

Asserting the existence of a relationship between the HCP's level of cultural competence and his/her ability to provider CCC, Campinha-Bacote (2002) put forward the PCCDHS model. A visual representation of the model is presented in Figure 1. The model, rooted in Leininger's transcultural theory, recognizes the fluctuating, dynamic nature of culture itself, and acknowledges the existence of intra-ethnic variations among 
cultural groups. To this extent, the model is based on several assumptions, including the understanding that cultural competence development is a process, not an end goal, and the persistence of a link between one's level of competence and their ability to provide CCC (Campinha-Bacote, 2002). The author surmises that one must be culturally competent in order to be able to provide effective and culturally responsive service to diverse client groups.

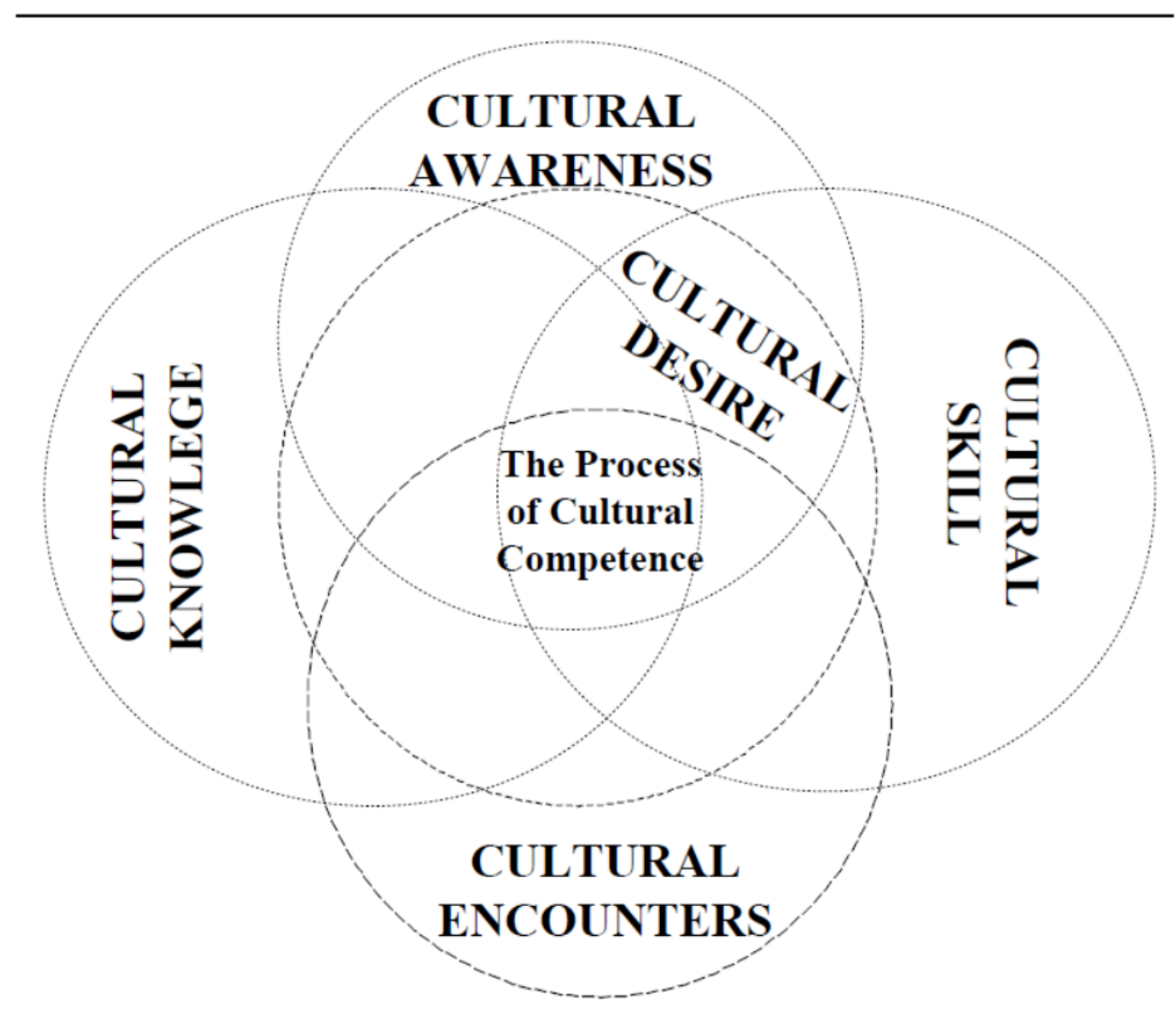

Figure 1: Campinha-Bacote's Model

As mentioned above, cultural competence is conceptualized by Campinha-Bacote as an ongoing process, whereby the provider focuses on becoming, rather than being, culturally competent. Being culturally competent has the connotative meaning that one has arrived at the end of their journey of competency development; the model, however, recognizes that culture has a dynamic nature. There is no single point at which the 
individual reaches full cultural competence (Shen, 2004). Since culture continues to undergo transformations, it is not stagnant, nor does it reach a plateau. The HCPs, therefore, must continue to evolve their understanding and abilities to provide CCC. Furthermore, as new elements of culture come forth, the HCPs must accordingly develop within themselves the competency to address those emerging changes.

\section{Constructs of Cultural Competence}

Campinha-Bacote's (2002) model postulated that building competence in any one of its five constructs - namely cultural awareness, cultural knowledge, cultural skills, cultural encounters and cultural desires - contributes to an overall improvement in cultural competence, however, all five of the constructs must be experienced, and internalized, in order for one to be able to provide quality CCC. Tortumluoglu (2006) emphasized this interdependence of the model's constructs, writing that all five must be addressed during the competence building process. Campinha-Bacote provided support to this notion through related publications detailing the effect that mastery of individual constructs has on client care. For instance, in her work on person-centered care and cultural conflict, Campinha-Bacote (2011) attested that the nurse's ability to conduct a cultural assessment, a task integral to CCC since it helps uncover the cultural preferences of the client, is aided by building on cultural skills and cultural encounters, two individual constructs of the model. The nurse becomes efficient at systemically evaluating the cultural beliefs, practices and values of clients through interactions with culturally diverse populations, and is able to engage the clients and their families in the decision-making around the culturally appropriate treatment options. Campinha-Bacote (2011) explained that extended proficiency of cultural skills and frequent cultural 
encounters may assist the nurse in providing $\mathrm{CCC}$ in instances where cultural conflict exists between the values, beliefs and ideas of the client and the healthcare principles. The nurse understands that care is not a one-sided win/lose situation, but that both she and the client will need to make compromises that are respectful of the client's cultural identity. Lacking in either one of these abilities would diminish the effectiveness of the other, resulting in poor quality of CCC.

Despite the interconnectivity of all five of the constructs of cultural competence in Campinha-Bacote's (2002) model, each has its own unique attributes and function in the competence development process. Cultural desire is the central construct as its spiritual undertones motivate the individual to voluntarily move forward in her journey to achieving cultural competence (Shen, 2004). In describing cultural desire, CampinhaBacote referred to the genuine passion one has for wanting to understand the client's culture in order to be able to provide care that is respectful of and congruent to the client's preferences. In other words, the individual has to want to provide CCC. In identifying the root of cultural desire, Tortumluoglu (2006) hypothesized that the individual HCP comes to recognize his/her lack of cultural incompetence "by attending workshops on cultural diversity, reading articles or books on the topic, or having direct cross-cultural experience with patients from culturally diverse backgrounds" (p. 8). The $\mathrm{HCP}$ then begins to understand that culture is important in healthcare, but does not yet know how to use this knowledge (Campinha-Bacote, 2002; Tortumluoglu, 2006). As the result of cultural desire, the individual feels driven to achieve cultural awareness and cultural knowledge, engage in cultural endeavors and develop cultural skills (Shen, 2004). Development of the remaining constructs, therefore, stems from cultural desire. 
In her model, Campinha-Bacote (2002) also presented explanations for each of the remaining four constructs. Having the capability to capture the cultural context of an individual's health and illness as part of cultural assessment is an example of cultural skill. Cultural encounters refer to the interactions with representatives of diverse cultural backgrounds. Cultural encounters have a twofold purpose, explained Campinha-Bacote: Firstly, engaging in verbal and non-verbal interactions with individuals of different cultural backgrounds builds the nurses' behavioral "vocabulary", meaning s/he becomes increasingly adapt at responding appropriately to culturally diverse contexts. Secondly, frequent and mindful interactions, whereby the nurse is conscious of the elasticity of culture (elasticity refers to the idea that two individuals who share a cultural background may respond differently to the same situation due to individual and/or contextual reasons) help accordingly validate or modify the nurse's values, beliefs and understanding of culture, consequently reducing the likelihood of cultural stereotyping. Cultural awareness refers to the individual's self-examination of cultural values, beliefs and expectations, both personal and professional. Having an understanding of one's own assumptions about culture reduces the likelihood that the individual will attempt to impose those beliefs onto others. Cultural knowledge, contrary to cultural awareness which is born out of personal reflection, acknowledges that one may learn about other cultures through structured educational ventures. The HCP is then able to understand the cultural values driving the clients' beliefs related to their health.

As Tortumluoglu (2006) concluded, the model can be used in the care for all people. At the heart of the model is the underlying understanding that the basic needs of people are the same across all groups; the expression of these needs, however, may be 
shaped by the cultural background of the individuals (Campinha-Bacote, 2002). By incorporating into their care routine the client's preferences that are born out of a unique cultural background, the HCPs are able to provide CCC. The highly abstract nature of the constructs identified in Campinha-Bacote's model, according to Brathwaite (2003) allows for it to be used across different segments of healthcare, including research and policy development, education, administration and clinical practice.

\section{Critical Appraisal of the PCCDHS Model}

The PCCDHS model emphasizes critical thinking, awareness, reflection and respect for individuals' uniqueness as being essential to the development of cultural competence (Dudas, 2012). Rumay Alexander (2008) specified components of active learning, as well as remodeling of skills and attitudes, as being integral to the development of cultural competence. Campinha-Bacote's (2002) conceptualization of cultural competence as an ongoing process, rather than a definitive end-point, exemplifies the active learning process; cultural competence does not develop by default, but is rather an intentional undertaking that comes from personal desire to improve (Dudas, 2012). The goals of developing cultural competence, according to the PCCDHS model, are to increase equity, reduce disparity and improve health outcomes (Tortumluoglu, 2006). In support of this, the model acknowledged the existence of variations between, as well as within, cultures (Campinha-Bacote, 2002).

The abstract nature of each of the individual constructs of the model makes it applicable across the healthcare spectrum (Tortumluoglu, 2006). For example, nurse educators can incorporate the individual constructs into the curriculum as a way of teaching student nurses how to deliver CCC. The individual constructs, as Tortumluoglu 
wrote, are logically congruent with each other and demonstrate clinical utility as they are related to the traditional worldview promoted in nursing. For instance, the model acknowledges the existence of multiple realities, as it stipulates that knowledge can be gained through a number of sources, including skills acquisition, medical anthropology, multicultural counseling and transcultural nursing (Tortumluoglu, 2006).

It is important to note that there have been limitations identified with the PCCDHS model, in particular those associated with the abstract nature of the construct cultural skill. Almutairi, McCarthy and Gardner (2015) conducted an in-depth exploration of the notions of cultural competence among non-Saudi Arabian nurses $(n=24)$ employed in hospitals in Saudi Arabia. The authors pointed out that CampinhaBacote (2002) conceptualized cultural skill as tasks inherent to nurse-patient interactions. In contrast, Almutairi et al. (2015) argued that cultural skill may also develop through interactions with other HCPs, not just via interactions with clients. Furthermore, the colleagues explain that the individual's environment (i.e. workplace settings) may influence one's development of cultural skill, therefore, others play a part in one's development. Campinha-Bacote (2002), however, maintained that the HCP holds the primary responsibility for their own development. Overall, however, the model aligns closely with the expectations of the healthcare field in general, and reflects the growing precision of the nursing profession's understanding of the multi-factorial and complex nature of CCC.

\section{The Current Landscape of CCC}

While the notion of cultural competence continues to gain recognition in the field of healthcare as a precursor to better client outcomes, it is yet to be fully integrated into 
the delivery of care (Lowe \& Archibald, 2009). Before I can explore the experiences of frontline staff of mono-culture LTCHs, it is imperative to uncover the influences currently shaping the landscape of CCC. The initial understanding of cultural competence as an element of person-centered care is introduced first, with reference to Tom Kitwood's (1997) work on individualized dementia care. Through the review process it was established that the predominant focus of literature on $\mathrm{CCC}$ revolves around the obstacles to successful integration of culturally appropriate clinical practices into healthcare settings. Factors associated with CCC at the level of the HCPs, educational institutions, and the healthcare organizations are presented below. Additionally, the literature focusing on the experiences of older immigrant adults with CCC is described.

\section{Cultural Competence and Person-Centered Care}

The concept of cultural competence aligns closely with nursing's mandate to provide person-centered care (PCC); the Ontario Ministry of Health/Long-Term Care has included the expectation of PCC in their health care regulations (MHLTC, 2010). Unlike the traditional biomedical approach to healthcare, PCC acknowledges factors external to the physiological well-being of an individual, such as lived experience, as having influence on the health of that client (Cooper Brathwaite, 2006; Suhonen, Alikleemola, Katajisto, \& Leino-Kilpi, 2011). PCC is more of a philosophy than a unified model, as it recognizes that all care occurs within the context of interpersonal relationships (Brownie \& Nancarrow, 2013). Cultural competence can, therefore, be viewed as a grounding element of PCC whereby the person's needs, values and beliefs are considered aside from a unilateral focus on the illness, and in such a manner, both the individuals' and the HCPs' agendas are integrated into the treatment schedule (Saha et al., 2008). The 
fundamental distinction between PCC and cultural competence is that the first aims to provide individualized care, placing greater emphasis on personal relationships between the individuals and the care providers, while the latter is primarily focused on increasing health equity and reducing disparities for the disadvantaged groups, including ethnic and cultural minorities (Campinha-Bacote, 2011). The two views, however, are complementary to each other. By providing $\mathrm{CCC}$ that demonstrates respect for the clients' cultural background, including lifestyle, values, beliefs and behavior, the HCPs adopt a person-centered approach in their practice.

Some of the earlier understanding of PCC comes from Tom Kitwood's (1997) work with dementia care in LTC settings. In his book Dementia Reconsidered: The Person Comes First, Kitwood described how he came to recognize that the traditional paradigm of dementia care approach was negatively oriented at emphasizing the limitations of the client demographic. According to Kitwood, the existing focus of care for people with dementia in the early 1990's was based on the biomedical understanding of the condition, which omitted the psychosocial influences that shape one's experience with illness. The care practices were so narrow in their focus on the medical needs, explained Kitwood, that they would deprive the person of human contact, while promoting an environment of disempowerment, objectification and intimidation. Kitwood noted that the tendency of depersonalizing individuals with a serious disability was a common practice among the different cultures. Such a negative approach is disrespectful towards the unique identity of a person, which arises based on lifestyle, outlook, beliefs, values and other related dimensions. Who the person is also depends on their life history. Understanding the personality of the person with dementia is essential to care, since 
personality tends to remain relatively preserved, even when the mental state has been altered due to illness (Kitwood, 1997). Kitwood, however, made no mention whether culture is an element of personality.

Through his writing, Kitwood (1997) affirmed the importance of an individualized approach to care and introduced the concept of personhood. Personhood refers to the bestowing of a status onto one person by another (Kitwood, 1997). In care of persons with dementia, Kitwood explained that by acknowledging a long-term care resident's personhood a staff member reinforces the resident's humanity through dignity, respect, trust and recognition. Promoting the individual's personhood, according to Kitwood, means acknowledging that the inherent human value of that person has remained intact despite their illness. Given how closely the focus on the individual's values mirrors the principles of individualized care promoted through PCC, I, as a researcher, was curious to understand how personhood deals with the element of culture. In reviewing Kitwood's work, however, it became apparent that the element of culture does not emerge through his writings. While he mentions the importance of exploring the values and beliefs of a resident, he does not explicate the importance of developing a deep understanding of the specific ethno-cultural practices of the resident. The LTC sector, however, including the Ontario Nursing Home Care Act (2010), stipulates that personhood is critical to the delivery of quality care. In recognizing the influence culture has on one's experience of health, and the limited focus on culture within current LTC models, I was compelled to wonder how CCC is conceptualized by the frontline care providers who work with clients in culturally specific care settings. 
Nursing professionals and healthcare organizations worldwide have recognized individualized care approaches such as PCC as being essential to the care of older people (International Council of Nurses, 2006). It has been positively associated with improved clinical outcomes (Suhonen, Valimaki, \& Leino-Kilpi., 2008) and falls prevention (Huang \& Acton, 2004) among older adults. Adopting a PCC approach in LTC setting means considering the residents' needs from a holistic perspective; the resident is then acknowledged as an autonomous and unique person (Chappell, Reid, \& Gish, 2006). By recognizing that the clients' past life experiences have shaped their present needs, care providers are able to develop care routines that mirror the lifestyle preferences of the residents (Gnaedinger, 2003); this, in turn, may reduce stress and anxiety associated with the move into a LTCH. There is, however, limited research on the adaptation of individualized, culturally oriented approaches, or previous cultural practices into the care of older adults in LTC settings (Suhonen et al., 2011), especially those of immigrant background.

Robinson and Gallagher (2008) explained that the introduction of a PCC approach into LTC institutions is a gradual process; the LTCHs, in the meantime, continue to operate in a manner similar to hospital settings. The culture change movement initiated as a result of the PCC philosophy has called for a reorganization of LTC homes to create homelike environments for residents that, in additional to physiological needs, can also meet their social demands (Caspar, O'Rouke, \& Gutman, 2009). Both the Ministry of Health and Long-Term Care (2011) and the Canadian Healthcare Association (2009) are proponents of the reorganization of LTCHs towards implementing a more homelike environment and of providing increasingly individualized care. Individualized care is 
emphasized in the LTCH Act (2010) with respect to medical directives and orders, bedtime and rest routines, personal care, continence care and bowel management, therapy services, and menu planning. An individualized approach to care is, therefore, on its way to becoming the gold standard.

\section{Healthcare Providers: Personalities \& Biases}

When compared to other HCPs, nurses are frequently the first line of contact for individuals entering the healthcare system and, since they spend the most time with the clients, they are more likely than any other caregiver groups to interact with them on an interpersonal level (Healey \& McKee, 2004). It is through these interpersonal encounters that a nurse has the opportunity to both gain a greater understanding of the client's cultural preferences and provide CCC (Seaton, 2010). Nurses, however, have identified lack of knowledge of cultural differences as the primary barrier to their ability to provide CCC (Cortis, 2004). Further review of the literature confirms the existence of a gap between the HCPs' knowledge of other cultures and their ability to purposefully and consistently enact care approaches that are sensitive to the clients' cultural backgrounds (DiCicco-Bloom \& Cohen, 2003; Jones, Cason \& Bond, 2004; Parker \& Geron, 2007; Taylor \& Alfred, 2010). A combination of personal factors and contextual influences affect the HCP's ability to provide CCC; this includes extent and scope of clinical experience, level of education, communication discrepancies, prejudice and ethnocentrism, and limited organizational support (Cooper Braithwaite, 2006; Facione \& Facione, 2007; Lipson \& DeSantis, 2007; Parker \& Geron, 2007; Reimer Kirkham, 1998). 
A number of the identified factors associated with lack of $\mathrm{CCC}$ have been also found to persist among nurses in Ontario. Cooper Brathwaite (2006) presented a substudy of a larger study of characteristics of public health nurses on the acquisition of cultural competence in response to an educational intervention. A one-group repeated measures design quantitative study $(n=76)$ was conducted in Southern Ontario with data being collected twice prior to and twice post intervention implementation. The intervention itself was presented over a course of five consecutive weeks during a twohour workshop; each session corresponded to the elements of Campinha-Bacote's (2002) model of cultural competence and gave the participants the opportunity to engage in reflective discussion. Additionally, a booster session was offered a month later for review purposes. According to the study findings, the length of professional experience and educational background collectively impact the nurse's ability to provide CCC.

Interestingly, Cooper Brathwaite (2006) reported that, in comparison to the more experienced nurses with lower levels of education, the public health nurses with fewer years of professional experience, but more advanced levels of education, had demonstrated higher levels of attained cultural competence after completing the multimodal educational intervention. Cooper Brathwaite (2006) presented a two-fold explanation for the findings of her study. Firstly, she suggested younger nurses may have had greater exposure to cultural training in their undergraduate programs, which predisposed them to some understanding of the concepts. Cooper Brathwaite (2006) also concluded that, as novice practitioners, younger nurses were more receptive to learning the new interventions than their more experienced, yet older professional counterparts. The facts, however, remain the same: although most HCPs are willing to and are 
interested in providing CCC, they lack the knowledge, skills and organizational support, such as availability of training, to be able to do this (Schim et al., 2007).

Nurses' perspectives: Caring for culturally diverse persons. As part of the discussion around CCC, it is important to include the perspectives and experiences of nurses caring for the culturally diverse client population as part of the analysis of the current landscape of the phenomenon. According to past research, personal factors, such as open-mindedness (Bonaparte, 1979) and degree of self-efficacy (Bernal \& Froman, 1993; Coffman, Shellman, \& Bernal, 2004) affect the nurses' attitudes towards providing CCC to clients from diverse cultural backgrounds. A further search of the literature yielded a small-scale exploratory research study by Boi (2000) of the experiences of nurses $(\mathrm{n}=7)$ in providing care to culturally diverse persons on a surgical ward at a hospital in London, Great Britain. Having the nurses reflect on their experiences of providing care to individuals whose cultural backgrounds differed from their own, Boi identified four common themes including issues related to communication, cultural differences, patients' relatives and nurses' education and training. The leading issues poor communication due to language disparity and inadequate knowledge of the clients' cultures - were flagged by the participants as barriers to providing safe and effective care. As part of her conclusions, Boi (2000) dispensed with the notion that every nurse must be an expert on all cultural groups, as it is a rather unachievable goal. Instead, Boi wrote nurses must demonstrate "cultural flexibility", or the ability to value all cultures. She supports the need for additional training and courses on CCC for practicing nurses.

Although the sample size of Boi's (2000) study was relatively small which limits the generalizability of the findings, additional international research was identified that 
similarly considers cultural competence in healthcare from the perspective of nurses. Applying the qualitative approach to yield contextual understanding of the participants' experiences, Leishman (2004) had interviewed ten nurses in Scotland, all of varied clinical backgrounds, on their experiences of caring for culturally diverse clients. Two themes emerged from the data of this study, including knowing about and learning cultural awareness, and experiences of caring for people from different cultures. With respect to the first theme, the participants explained they had received none-to-little training on cultural awareness, although all ten had unanimously identified cultural competence as a necessary nursing skill. When discussing their experiences of caring for people from different cultures, the participants admitted to having limited experience and education in this area, although they were aware of the need to provide individualized care to clients. Primarily, the nurses focused on exhibiting sensitivity and respect in their interactions with culturally diverse individuals. The data from this study reinforced the gap that persists in the nursing school curricula with respect to CCC. However, the participants' comments did demonstrate willingness to build on the skills associated with cultural competence.

Not all HCPs, however, have a positive attitude towards CCC like the participants of Leishman's (2004) study. Home case nurses participating in DiCicco-Bloom and Cohen's (2003) study were generally found to disregard client's cultural differences during their interactions. The colleagues assessed, through observation and interviews, the culturally mediated interactions between 14 client-nurse home care dyads. In their practice, the nurses were observed analyzing the cultural conflict between themselves and the clients through an ethnocentric lens, meaning they would evaluate the clients' 
experiences with respect to their own cultural norms. A direct example of this practice is a case described by DiCiccio-Bloom and Cohen of an elderly Russian client who did not speak English. Even though the English-speaking nurse had recognized the language barrier, she faulted the client for not arranging for an interpreter and continued to provide health teachings to the client in English. The client was then discharged early. The authors explain, "This [case] was an example of how culturally inappropriate care acted as a barrier to access to health care services and could lead to poor health outcomes" ( $p$. 28). In fact, the nurses were observed acknowledging the client's cultural difference in only 3 out of the 14 dyads. However, in all three instances the nurses encountered resistance from their supervisors whenever they would suggest approaches to improve the outcomes of their culturally diverse clients. DiCicco-Bloom and Cohen (2003) concluded that the participating nurses, as well as their management, need to build on their skills of providing CCC.

In summary, when considering nurses' practice of $\mathrm{CCC}$, due to the nature of their job, nurses spend extensive amount of time interacting with clients on daily basis. It, therefore, falls upon the nurses to provide CCC demonstrating respect for their unique needs. The three studies highlighted herein that considered the experiences of nurses with providing $\mathrm{CCC}$ to ethnically diverse client population were conducted internationally (Great Britain, Scotland, United States); additionally, none of the studies took place in a LTC setting. Given the limited availability of research detailing the experiences of nurses of providing CCC to diverse client populations, it is becoming more apparent that there is justification to this researcher's aim to explore the perceptions of frontline staff in Ukrainian-specific LTCH of providing CCC to older Ukrainian immigrant residents. 


\section{Policy Makers and Focus on CCC}

Although a number of local initiatives are in place in the province of Ontario addressing the needs of the province's diverse population, the consistency of the content on cultural competence is varied among the social services spheres. With respect to the healthcare sector specifically, Ontario currently does not have standardized training initiatives for teaching cultural competence to HCPs, although independent and fragmented actions have been taken by various subsectors to address the issue. One such example is the document titled Embracing Cultural Competence in Mental Health and Addiction System (2009); compiled by the Ontario Federation of Community Mental Health and Addiction Programs it recommends that multidisciplinary healthcare teams undergo training to improve knowledge and attitudes associated with cultural competence. The content of the document, however, is predominantly targeted for HCPs working in mental health and addiction services. Although the key concepts of cultural competence addressed in the document are transferable to other sectors within the healthcare field, the specificity of the documents' recommendations make it a challenge to implement anywhere else aside from mental health and addiction services. Another example of an educational initiative addressing cultural competence of HCPs comes from the training developed by the Association of Canadian Academic Healthcare Organizations and the Canadian Healthcare Association. It is an online course titled Cultural Competence \& Cultural Safety in Health Program, which primarily addresses building cultural competence skills in caring for First Nation, Inuit and Metis peoples. Similar to the document on mental health and addiction services, this online course focuses on the cultural needs of a specific population group, which restricts its applicability in care of other cultural groups, including older Ukrainian immigrant adults. 
Professional healthcare associations, however, acknowledge the importance of cultural competence among their members. For example, the RNAO (2007) advocates for cultural competence and respect for diversity in its Best Practice Guidelines. Similarly, in fulfillment of their vision to promote excellence in nursing practice in the province, the CNO (2009) has released a practice guideline titled Culturally Sensitive Care, which details the assumptions around cultural competence, elements of providing culturally sensitive care, and suggests approaches for developing culturally sensitive care. Finally, the Canadian Nurses Association (2010) has put forward a position statement identifying cultural competence as an entry-to-practice level competence for nurses. It outlines the responsibilities of nurses, employers, educators, regulatory bodies and nursing unions for promoting and integrating cultural competence into the care of culturally diverse clients. The wide acknowledgement of cultural competence by the professional associations further attests to the relationship of CCC to health outcomes of the client population.

Despite the strong support from professional bodies, there is discrepancy in the implementation of cultural training initiatives at the level of healthcare facilities, in particular in Ontario's LTC sector. While the funding for LTCHs is allocated from the provincial budget, it is at the discretion of the care facilities what fraction of money is directed at improving CCC among the staff members. The LTCH Act (2010) stipulates training and orientation programs are to be available and implemented for staff; HCPs providing direct care are to be trained in specific areas, including fall prevention and management. Training in CCC, however, is not among the list of educational areas identified by the Act, which raises concern as to the amount of attention that is directed at improving the cultural competence capabilities of HCPs in LTCHs. Such delimitations of 
training initiatives create the potential for minimal funding to be directed towards training and supporting the staff along their journey of developing cultural competence, as this is not one of the areas identified by the Act. This is especially a concern in Ontario where the Residents' Bill of Rights (2008) document outlines the residents' rights during their stay at the LTCH, however, there is no mention of standards around CCC. The Bill simply acknowledges, "every resident has the right to pursue social, cultural, religious, spiritual and other interests" (p. 24). There is ambiguity as to how this Right is to be implemented in LTCHs and what responsibility is held by the practicing nurses to ensure CCC is delivered.

Efforts related to promoting cultural competence among health and social services have also been carried out at the national level, yet they are narrow in their grasp and specific to a cultural and/or ethnic group, instead of being applicable cross-culturally. This is similar to the training initiatives mentioned earlier, which target either specific healthcare settings or cultural groups. An example of a national level framework is a publication by the Aboriginal Nurses Association of Canada (2009) titled Cultural Competence and Cultural Safety in Nursing Education: A Framework for First Nations, Inuit and Metis Nursing. As the title suggests, the framework is primarily aimed at strengthening the human support resources for First Nation, Inuit and Metis populations in Canada. Further research is needed to identify the essential components of CCC for other cultural groups represented among the Canada's population, including older Ukrainian immigrants. Such understanding may then be integrated into developing a national framework on CCC that can be applied cross-culturally, as well as in culturespecific LTCHs. 


\section{Immigrant Status: Culture and Health Outcomes}

According to the literature, seniors of immigrant background are more likely to have restricted access to healthcare and to present with poor health outcomes (Pitkin Derose, Escarce, \& Lurie, 2007). This holds true in the Canadian context, as Newbold and Danforth (2003) have deduced from the findings of the 2000/2001 Canadian Community Health Survey that immigrants are more likely to report worse health status in comparison to Canadian-born peers. Another case-in-point scholarly example of the lower quality of perceived health among Canada's senior immigrants comes from the findings of a Manitoba-based study by Menec, Schooshtari, and Lambert (2007). The colleagues had conducted a cross-sectional and longitudinal study ( $\mathrm{n}=4559$ in 1983 and $n=1361$ in 1996), the result of which indicated that older adults of Eastern-European descent (including Ukrainian, Russian and Polish immigrants) are less likely to rate their health as good/excellent when compared to their British/Canadian-born counterparts. The differences were not eliminated after the researchers controlled for demographic variables, language spoken, and socioeconomic and health statuses. As the result of their findings, the researchers concluded that cultural factors play a part in people's self-rated health. Further to this, Menec and colleagues acknowledged that cultural influences on health last regardless of the length of the person's settlement in Canada, and may even persist in subsequent generations. This echoes Falicov's (2005) writings that cultural norms and a connection to the place of origin are preserved into the second and even third generations. Culture, therefore, demands attention as a determinant of health outcomes in the care of immigrant populations and their descendants.

Social, interpersonal and institutional factors have been noted to affect the health and well-being of senior immigrants. Language disparity, social class standing, and the 
structure of the healthcare system have been associated with poor health outcomes of older immigrant groups in the developed countries in the West; additionally, HCPs with limited cultural awareness, along with interpersonal and institutional proclivity for stereotyping based on cultural differences, further restrict immigrants' access to appropriate healthcare (Eggenberger, Grassley, \& Restrepo, 2006; Majumdar et al., 2004; Markova \& Boome, 2007; Ortiz \& Fitten, 2000; Weinick, Zuvekas, \& Cohen, 2000). Collectively, these barriers are closely associated with culture, a concept earlier defined in this chapter as the values, beliefs and practices shaped by common experiences and transmitted through membership within a specific group (Purnell, 2002; Schim et al., 2007). Culture is ever-present and evolving; as such, it has the potential to direct people's health-related behaviors (Aroian, Khatutsky, Tran, \& Balsam, 2001; Steward et al., 2011). Consequently, a positive relationship has been highlighted in the literature between the availability of $\mathrm{CCC}$ and enhanced health outcomes of immigrant persons (Betancourt et al., 2005; Like, 2011). Collectively, the statistics given earlier, along with the findings on CCC presented in this section, reinforce the scholarly need to consider how HCPs integrate culture into their care of older immigrant adults, in particular those of Eastern European descent, who, although they make up a large and socially significant portion of Canada's population, have been given little consideration in healthcare research.

\section{Increasing Availability of CCC: Targeting Practice \& Education}

From the above-presented overview of the literature on CCC, a conclusion can be reached that enhancing the availability of $\mathrm{CCC}$ is essential for promoting better quality of care for the population as a whole, and for older immigrant adults specifically. 
Approaches to promoting $\mathrm{CCC}$ have been identified in both healthcare practice settings and education. In the pages that follow, the practice of ethnic match as a precursor to $\mathrm{CCC}$ is introduced and critiqued. Similarly, approaches for building capacity for CCC among nurses is explored from the perspective of education and training.

\section{Ethnic Match: Potential for Enhancing CCC}

In recent years, ethnic match has been considered as a potential approach for addressing the limited availability of CCC. As the name implies, the approach calls for the care recipient-HCP dyad to be paired up based on shared ethnic, racial and/or cultural affiliations. Heikkila, Sarvimaki and Ekman (2007) attested that attaining such cultural congruency "based on the residents' mother language, shared ethnic background with staff, and shared customs creates a common ground for communication and understanding", which, in turn, helps build caring relationships and promote client's wellbeing (p. 354). Stuart and Nielsen (2011), whose article, as was mentioned earlier, was the starting point for this study, believed that the match up between the care provider's and client's ethnic and cultural backgrounds is likely to result in higher quality of care. They were of the opinion that the nurse's introspective view into the history of the group's culture increases understanding of person's needs, promotes ease of communication and lays the foundation for trust, which is often difficult to establish in new client-provider interactions. Benkert, Peters, Clark, and Keves-Foster (2006) expect HCPs of similar cultural background would be granted a higher degree of trust by the members of their communities during clinical interactions, thus bridging the divide between the clients and the caregivers. It is anticipated that the practice of ethnic match can be facilitated by increasing the diversity of the healthcare workforce to better reflect 
the ethnic diversity of the general population; currently, however, Canada's healthcare workforce is commonly characterized as being culturally and ethnically homogenous (McGinnis, Brush, \& Moore, 2010; Gilchrist \& Rector, 2007). The diversification of the general population in Canada, notes RNAO (2007), is not reflected among nursing professionals (Canadian Nurses Association, 2004). Ethnic homogeneity of the nursing profession is associated with lack of cultural awareness, and discrimination and racism towards clients, all of which reflect poor CCC (RNAO, 2007). It is, therefore, worthwhile to further uncover the factors associated with ethnic match practices through the perspectives of frontline HCPs.

The ethnic match approach has been shown to be effective in promoting CCC in related social services. An ethnographic study from Sweden of a social day care program for Iranian immigrants demonstrated the positive effects of the ethno-cultural match between providers and recipients of social services (Emami, Torres, Lipson \& Ekman, 2000). Over the course of twelve months, the researchers had observed the 323 participants who had attended the center on a daily basis; furthermore, detailed interviews were conducted with 15 elderly Iranian immigrants from the program. Taking part in a program based on their cultural traditions and delivered by staff who share the participants' language created a strong sense of social belonging for the elderly Iranian immigrants. Among the study findings was also the theme cultural understanding, whereby having the day care staff speak the language of the participants was greatly appreciated. Overall, being cared for by representatives of their cultural background was found to be highly beneficial for the elderly Iranian immigrants participating in the day care program in Sweden. 
One of the elements used to determine ethnic match is speaking the same language. Sharing a language with the HCPs has been identified as a precursor to $\mathrm{CCC}$ among older immigrant adults by Heikkila et al.(2007). The colleagues had conducted a 13-months long focused ethnography study of a Finnish LTCH in Sweden with the aim of describing how CCC affects the well-being of older Finnish immigrants. The observational conclusions were augmented with information collected through interviews with nursing staff, residents and their visitors. Heikkila and colleagues reported that Finnish language was at the core of all interactions between residents and staff, allowing the residents the opportunity to express their preferences and needs directly to the staff. Other relevant findings included the following themes: shared language eases communication, shared languages eases care situations, shared cultural knowledge enables the individualization of care, and shared cultural knowledge creates solidarity between staff and residents. Collectively, these themes identified linguistic congruency between the care provider and recipient as the facilitator of a therapeutic relationship that promotes the client's well-being. When considering these findings related to language and ethnic match, it is also important to keep in mind that culture encompasses other elements, including social, demographic and political background. Language, however, consistently appears as the predominant identifier of cultural affiliations.

\section{Ethnic Match: The Opposing Views}

Some researchers have questioned the appropriateness of the ethnic match as a facilitator of CCC, recognizing that factors beyond the ethnic or cultural overlap can influence the healthcare experience (Schim et al., 2007). For instance, McGinnis et al., (2010) noted that age, education, gender, life experiences and professional occupation 
may all impact, in some capacity, the client's relationship with the HCP. Even in instances when the HCP and the client share a common ethnic or cultural background, they may enter the clinical encounter with different ideas and perceptions, since diversity exists within cultural groups as often as it does among cultural groups (Bonder, Martin \& Miracle, 2001; Waxler-Morrison, Anderson, Richardson, \& Chambers, 2005). This point has been emphasized earlier in this chapter. This leads to the conclusion that pairing clients and HCPs according to ethnicity and culture may result in unexpected tensions; while the two may share some common elements, such as language, differences in socioeconomic status, age and gender may predominate (Waxler-Morrison et al., 2005). Inter-generational gaps, socioeconomic status, along with traditional gender roles and expectations may contribute to diverging enactment of cultural elements among the different members of the same cultural group.

The effectiveness of the ethnic match approach has been critically evaluated among related professional groups in healthcare, including therapists. Based on research from the 1990s that asserted ethnic match to be associated with more favorable treatment outcomes for therapist-client dyads, Zane et al. (2005) investigated the outcome of cognitive match between White $(\mathrm{n}=27)$ and Asian $(\mathrm{n}=33)$ outpatient clients and their therapists $(n=18)$. The data collected included demographic variables, perceptions of the problem, coping orientation, and treatment outcomes. The colleagues report that clienttherapist cognitive match (i.e. when both parties had similar expectations of the treatment or coping strategies) held stronger value for the client, even after controlling for ethnic match and language preference. Although the findings did not discredit the validity of ethnic match-up as a potential facilitator of positive treatment outcomes, they did show 
that other factors may have an influence. The study, however, had several limitations, including the small sample sizes of the various Asian American and White American ethnic group, which impede generalizability of findings. Additionally, as the authors point out, the study was prospective in nature, therefore, other variables aside from ethnic or cognitive match may affect the study outcomes. There is, consequently, value in directing research efforts at understanding the experiences of ethnic match practices within healthcare settings to uncover further elements of interest.

When examining the appropriateness of an ethnic match between the care provider and care recipient, it is vital to consider the preferences of the latter as part of the individualized care paradigm. Hikoyeda and Wallace (2001) explored this by conducting a qualitative inquiry that compared the experiences of twelve Japanese American women living in culture-specific facilities to the experiences of fourteen Japanese American women in non-Japanese residential care facilities for the elderly. At the time, this study was considered trailblazing research that examined the satisfaction with LTC services from the perspective of ethnic elders. Hikoyeda and Wallace (2001) found that Japanese American female residents in LTCHs did not exhibit particular preference for caregivers who share their ethnic background. The residents identified Japanese staff, in accordance to the group's cultural norms, to be more reserved in their approach, while labeling the friendliness of Caucasian English-speaking staff as "artificial". Instead, the residents voiced preferences for receiving care from staff exhibiting such general characteristics as kindness, friendliness, patience, empathy, helpfulness and respect. The residents' family members $(n=31)$ spoke more frequently of their preference to have their family members looked after by care providers of similar 
ethnic and/or cultural background. While these findings highlight the discrepancy between the residents' and family members' preferences for characteristics of LTCH staff, it is important to note that Hikoyeda and Wallace (2001) gave limited demographic data on the participants; hence, the length of residence in United States (US) and level of acculturation, both of which may impact on participants' experiences, are not explicated. Further consideration needs to be given to the relationship between CCC and the ethnic match approach.

\section{Cared for by Culturally Diverse HCPs: Clients' Perspectives}

In exploring the potential of ethnic match as a viable approach to promoting $\mathrm{CCC}$, valuable insight can be gained from considering the perspectives of culturally diverse care recipients on receiving care from HCPs whose cultural background differs from their own. For instance, as recently as 2009 , more than $8 \%$ of nurses employed in Canada were internationally educated professionals (Horne, 2011). Canada's nursing workforce, however, has been described in the literature as being culturally homogeneous, which raises concern that the individual practitioners may be poorly prepared to care for the diverse client population that is emerging as the result of changes in the country's immigration patterns. Acknowledging that culture shapes people's worldviews translates into the understanding that nurses enter the clinical settings with their own set of cultural expectations and beliefs, which may impact the quality of care they offer. Cultural diversity, therefore, exists not only within the client population, but also among the HCPs themselves, and may serve as a point of divergence within the client-HCP relationship. This notion has been considered in the literature stemming out of the US, which, similar to Canada, is a multicultural nation. 
In exploring the effects of racial and cultural differences between nurse's aides and the residents of LTCHs in the United States, Berdes and Eckert (2001), identified instances of racism aimed at the racially diverse nursing aides' by the nursing home residents in 11 out of the 30 participant interviews. To this extent, the authors concluded that racial and cultural differences among the staff and residents in LTC facilities contribute to social distance between the two groups, and, as such, may negatively affect the residents' health outcomes. Identifying the potential for poor interpersonal compatibility between the HCPs and the residents as a result of varying cultural backgrounds, in conjunction with the cultural nuances of Canada's healthcare workforce, further highlights the importance of understanding how $\mathrm{CCC}$ is provided in instances where there is an ethnic match between the $\mathrm{HCP}$ and the client. The principles derived out of this understanding may then be applied to the care of other cultural groups.

In the meantime, much of the research around the clients' perspectives of what it means to be the recipient of $\mathrm{CCC}$ from culturally diverse HCPs has been conducted from the perspective of the patient-physician interactions. For example, aiming to contribute to the development of a culturally competent healthcare system in the US, Tucker, Herman, Pedersen, Highley, Montrichard and Ivery (2003) conducted focus groups with a culturally diverse group of primary care patients (52 African Americans, 38 European Americans, and 45 Latino Americans). Collectively, all three ethnic groups of participants identified positive personal qualities of the HCPs (i.e. attentive listening, effective communication, posing engaging questions), rather than the cultural similarity between themselves and HCPs, as key elements to CCC. Also, the participants spoke of the significance of an individualized approach as being a precursor to CCC; it was 
important for the individuals to feel that the physician understood them on a personal level.

The findings of Tucker et al.'s (2003) study further advance the proposition that matching the care providers and recipients based on cultural backgrounds is not always the optimal approach for ensuring CCC; rather, the culturally diverse participants identify general people skills, such as being polite and a good listener, as exemplifying CCC. It is interesting to note that only the Latino American participants had identified the sharing of a common language with the care provider as an element of CCC. This acknowledgement speaks to the importance of communication as a means of understanding and providing CCC among this ethnic group, although the study authors did not further explore why this is so. Still, language may sometimes be spoken by individuals who are not of the same cultural background as the clients. For instance, although different in dialect, the French language is commonly spoken by residents of France, Belgium, and a number of countries in Africa, all of which differ in other expressions of culture, including cuisine and religion. Language, therefore, while an integral element of culture, is not necessarily the only factor defining what it means to belong to a specific group.

The results of Tucker et al.'s (2003) study have been confirmed by NapolesSpringer, Santoyo, Houston, Perez-stable, and Stewart (2005). In this study out of US, the researchers had held focus groups with a total of 163 participants, including 61 African Americans, 45 Latinos and 55 non-Latino Whites, discussing the participants' understanding of culture and how it is incorporated into care by their physicians. From the data analysis, the colleagues concluded that culture plays a significant role in the medical encounters for the three ethnic groups of participants, although they did not find 
that sharing a cultural background is a mandatory requirement for CCC. A small number of African-American female participants had reported their preference for female physicians who shared their ethnic background. As the authors explain, the AfricanAmerican female participants felt that their African American female physicians could identify better with them. This preference for ethnic concordance was not repeated among the other groups of participants. Instead, the majority of the participants reported feeling more satisfied with their care when it was delivered by physicians who demonstrated strong interpersonal communication skills and understanding of such cultural elements as the significance of alternative medicine, spirituality and nutrition. The researchers, therefore, conclude that, in response to the ongoing diversification of healthcare client population, healthcare services need to focus on promoting practices and beliefs that are respectful of others' cultures. Matching up HCPs and clients based on cultural backgrounds, however, may be of benefit to some, but not all, culturally diverse healthcare clients.

\section{Nursing Education and Training: Improving Cultural Competence}

HCPs' limited ability to provide CCC leaves the clients at the receiving end of ethnocentric attitudes, misguided communication, inaccurate diagnoses and ineffective treatment interventions (Coffman et al., 2004). After completing a systematic review of thirty-four studies evaluating the interventions for improving cultural competence of HCPs, Beach et al. (2005) concluded that such training may enhance both the HCPs' knowledge, as well as attitudes and skills related to cultural competence. Furthermore, Beach and colleagues noted that cultural competence training has a positive effect on 
client satisfaction with their care. In this US-based literature review, 17 out of the 34 studies had focused on nurses.

Similar findings on the significance of education and training on CCC have been reported among Canadian HCPs. Results of a randomized controlled trial $(n=114)$ by Majumdar et al. (2004) on the effectiveness of cultural sensitivity training on HCPs' knowledge and attitudes revealed training to be an effective approach for increasing awareness, understanding of and communication among HCPs and minority groups. Data was collected from the experimental and control group at the start of the study, and subsequently at 3,6 , and 12 months post randomization. One of the strengths of the study was that it also involved patients ( $\mathrm{n}=133$ ) of the participating HCPs, who volunteered to assist in determining the effect of training. According to the results, the patients of the HCP participants who had undergone cultural sensitivity training demonstrated improvements in the utilization of healthcare resources and overall functional capacity at the one-year mark. Further studies are needed to evaluate whether training translates to behavioral changes in the long-term for the HCPs.

As a Canadian-based research, the data from Majumdar et al.'s (2004) study provided support for the development and implementation of education and training resources on CCC for HCPs. Related studies have shown that HCPs' cultural competence can be improved via varying education modes, including workshops (Cooper Brathwaite, 2005; Williams, 2002), immersion in the different culture of interest (Fehrenwald et al., 2001; Mac Avoy \& Torth Lippman, 2001; Walsh \& DeJoseph, 2003), and formal courses on CCC (Crandal et al., 2003; Diaz-Lazaro \& Cohen, 2001).There is, however, evidence of poor and varied integration of content on CCC into the undergraduate curricula of 
nursing schools across North America despite research that links culturally competent healthcare interventions with reduced health disparities among ethnic minorities (Lipson \& DeSantis, 2007; Long, 2012; Maddelena, 2009).

Sumpter and Carthon (2011) conducted a qualitative descriptive study of undergraduate $(\mathrm{n}=5)$ and doctoral level $(\mathrm{n}=5)$ nursing students at the University of Pennsylvania School of Nursing in United States with the aim of evaluating their perceptions of the integration of CCC content into the nursing curriculum. The study findings revealed that, while the students had exposure to content on cultural competence, the inconsistency in definitions of the concepts left the students questioning their ability to employ the principles of CCC in their future practice. Both bachelors and doctoral level student nurses were able to identify the opportunities missed by faculty that could have been used to present cultural competence content. The fragmented incorporation of content on CCC into the nursing education is confirmed by Betancourt et al. (2003) and Sargent, Sedlak, and Martsolf (2005). Further to this, Starr, Shattell and Gonzales (2011) explained that many nurse educators are intimidated by the responsibility of providing CCC training to student nurses.

\section{Chapter Summary}

Review of the literature has led me to conclude that CCC is a highly complex phenomenon, however, it has been flagged as a potential approach for reducing healthcare disparities faced by older immigrant adults. In order for CCC to be delivered by HCPs, the following elements are seen as fundamental: characteristics and skills of the HCPs, structural support, familiarity with culture and strong rapport among the clients and the HCPs. The review suggests that research around CCC has primarily focused on 
ethnic minority groups largely ignoring the heterogeneity that exists within the Caucasian segment of the population, specifically those immigrants who are of Eastern European descent. In addition, there is lack of theoretical clarity as to whether cultural values and beliefs within specific ethnic groups are acquired through experience or inherent in some way at birth, resulting in questions about how cultural competence can be enhanced when caregivers and recipients are from very different cultural backgrounds. Further to this, even among the fragmented body of literature available on the topic of cultural competence, there is lack of Canadian-based research.

This literature review identified a single publication involving Ukrainian participants in a study on the use of healthcare services by older refugees; however, the study was based in the United States and did not take place in the LTC context. This represents a significant gap in the literature. Therefore, this thesis focuses on the experiences with CCC of Ukrainian nurses, a group that shares the author's ethnic and cultural heritage, and has contributed exponentially to the social milieu of Canada. Given that cultural competence seems to have some link to inherent knowledge, and that there has been little research into the cultural competence of Ukrainian nurses, a significant gap in the literature has been identified, and this study is justified.

\section{Statement of Study Purpose}

The purpose of this narrative study is to understand the perspectives of nurses who provide CCC to older immigrant persons living in culture-specific LTCHs. As the research study was begun, $\mathrm{CCC}$ was generally defined as the actions, beliefs and attitudes that come together in an efficient way to consider and respect cultural diversity of the 
care recipients, thus ensuring better client outcomes (DiCicco-Bloom \& Cohen, 2003;

RNAO, 2007).

\section{Research Question}

How do nurses employed in Ukrainian-specific long-term care homes conceptualize and deliver culturally competent care to older immigrant adults?

The next chapter will take this research question and put it in the context of theory, methodology and design. 


\section{CHAPTER 3}

\section{Theoretical Lens, Methodology \& Design \\ Introduction}

The purpose of this study was to narrate the experiences of two frontline nursing staff of two Ukrainian-specific LTCHs in providing CCC to older immigrant residents of Ukrainian descent. The intent of this chapter is to give the reader insight into how the study was conducted. The concept of CCC, in this context, was studied using Lieblich, Tuval-Mashiach and Zilber's (1998) narrative methodology. In the following pages I share the theoretical lens and the philosophical assumptions that guided the study. I also detail the study design, including the data collection and analysis approaches. Efforts for promoting rigor in this narrative study and potential ethical concerns are also discussed.

\section{Theoretical Lens: The World Through a Thousand Eyes}

In approaching the development of the study design, I have defined my ontological assumption to be centered on the multimodality of reality. In choosing to carry out qualitative research, I acknowledge the idea of multiple realities (Creswell, 2007). Lieblich et al. (1998) acknowledge that qualitative researchers accept the notion that there is no absolute truth. As philosopher Descartes explained, a single moment may be viewed in a thousand different ways by a thousand different eyes (Cottingha, Stoothoff, \& Murdoch, 1985). It is, therefore, my intent to bring forward findings that reflect the realities as they are experienced and expressed by the study participants.

I approached this study already holding some preconceived notions of CCC. My understanding is grounded in literature on $\mathrm{CCC}$, as well as my own professional experience of caring for individuals of diverse cultural backgrounds. My ideas were shaped through reviewing literature that conceptualized CCC as referring to the actions, 
attitudes and behaviors of HCPs that exhibit understanding of and respect for the unique cultural backgrounds of the clients. This includes sensitivity to language, as well as customs and rituals guided by cultural group affiliations. CCC, I have come to believe, is able to resolve the disparity between cultural needs of the patients and the mandates of the healthcare system.

My philosophical assumptions are closely aligned with the social constructivist perspective, which is, therefore, used as an interpretive framework for this study. Social constructivism maintains that individuals develop subjective, context-shaped meanings of their experiences; culture, social interactions and historical developments are among the potential contextual influences (Creswell, 2007). Butts (2013) confirms that interactions with others, along with cultural norms and expectations, shape the subjective meanings of people's experiences. When trying to uncover the meaning of a phenomenon from the constructivist approach, the research methodology must center on the participant's world so that the findings are representative of the way the participant interprets her lived experiences (McEwen \& Wills, 2011).

The social constructivist standpoint that I ascribe to fits well with the current discourse around the definition of cultural competence. Traditionally, cultural competence has been considered from the essentialist perspective, which establishes culture as a stable and unchanging concept (Kirkmayer, 2012); CCC therefore would view culture as the differences between people, largely ignoring the dynamic and relational nature of this concept (Carpenter-Song, Nordquest Schwallie, \& Longhofer., 2007). The essentialist perspective, therefore, largely omits the social context and the power relations that affect CCC (Gray \& Thomas, 2006). The more recent perspective 
accepts that being aware of the differences that may persist between oneself and members of other cultures does not lay the foundation for CCC, although some developmental models of cultural competence are still based on this assumption (Garneau \& Pepin, 2015; Jenks, 2011; Williamson \& Harris, 2010). Garneau and Pepin (2015) warn that HCPs operating from the essentialist perspective may "see health problems as the result of cultural behaviors, instead of the results of other factors such as person's living conditions and socioeconomic factors" (p. 10). Applying cultural competence within the clinical setting is intended to improve the understanding, and consequently the relationship, between the care provider and recipient, yet the essentialist perspective perpetuates stereotypical views of different cultures (Browne et al., 2009).

An alternative viewpoint is needed in order to connect the modern holistic approach with the traditional medical model of care. To this extent I have adopted the definition of cultural competence as proposed by Garneau and Pepin (2015). Rooted in the constructivist perspective, it defines cultural competence as "a complex know-act grounded in critical reflection and action, which the health care professional draws upon to provide culturally safe, congruent, and effective care in partnership with individuals, families, and communities living health experiences, and which takes into account the social and political dimensions of care" (Garneau \& Pepin, 2015, p.12). This definition strongly reinforces Guba and Lincoln's (2005) position on the existence of multiple, socially constructed realities in that it views culture as relational to, and under the influence of, historical context. It also encourages HCPs to bring critical thinking into their relationship with the clients and to engage in interpersonal exploration, through dialogue, to better understand the perspective of the clients (Garneau \& Pepin, 2015). 


\section{Applying Theory and Philosophy to Real Life}

Acknowledging that there is no single universal truth raises my awareness to the fact that the knowledge derived from this study will be positioned from the subjective perspective of the participants. The participants shared with me the truth as they experienced it. It was essential for me to foster the development of a positive rapport with the participants so that they felt as comfortable as was possible for them in sharing their narratives in an unrestricted manner. As emphasized by Josselson (2007), the nature of the data collected through a narrative study largely depends on the relationship of trust and empathy the interviewer establishes with the participant. Rather than solely answering the explicit interview questions, the participants also respond to the interpersonal cues demonstrated by the interviewer, including compassion, understanding, tolerance, and a non-judgmental outlook (Josselson, 2007). To fulfill my obligation to the participants as the researcher, I maintained a professional demeanor throughout the interviews and responded to emotion-laden discussions with empathetic understanding. I also made the effort to build trust between myself and the participants by following through on the promises I made, both verbally and in writing.

An example of my efforts to maintain trust comes from interview one with Participant One. The participant left the room at the end of our first interview before I had the chance to give her a gift card to a coffee shop. The recruitment poster for the study stated that the participants would be compensated for their time with a gift card. Similarly, the Information and Consent Form that Participant One had signed stated that the gift card would be provided at the end of interview one, regardless whether the participant chose to continue with the study or withdrew from it. As I realized that I had not given the participant the gift card, I made my way outside and caught up with the 
participant prior to her leaving the meeting location. I apologized for the oversight and insisted that she take the gift card. She accepted my apology and the card, then assured me that her decision to participate in the study was not related to the promise of the gift card. By including the information about the gift card on the poster and in the form, I made a commitment to the participants. Had I failed to follow through on my promise to participant one it could have jeopardized the trust the participant had in my ability to protect her identity and may have negatively affected the quality of the narrative.

\section{Methodological Approach: Nurses \& Storytelling}

Among other qualitative research approaches, narrative, with its focus on storytelling, fits closely with the aim of this study, which is to understand the personal and storied experiences of nurses in a Ukrainian-specific LTCH of providing CCC to older Ukrainian immigrants. There is a congruence between the nursing practice of using storytelling during patient care encounters and the defining characteristics of narrative research, as both are concerned with understanding reality as it is experienced by the individual and transmitting its meaning to others (Frank, 2006; McCance, McKenna, \& Boore, 2001). Patients and nurses tell stories in order to identify, formalize and share their own meanings. Patients use storytelling to derive meaning of their illness, while nurses reveal the meaning of nursing care through their own narratives (Frank, 2006; McCance et al., 2001). Narrative can, therefore, be both a therapeutic tool, a professional development initiative or a phenomenon under study (Latimer, 2003; Litchman, 2013). People use narrative stories to make sense of the world around them, as well as crystallize their emergent self-identity, refining it with time and in response to new experiences (Mishler, 1986; Reissman, 1993). In this manner, narrative research conducted from the 
social constructivist perspective, as I have adopted, facilitates uncovering the context of the individuals' experiences. Reissman (1993), however, warns the interpretive researcher that the focus of an individual's narrative should not be its historical accuracy, but rather the meaning that the storyteller wants to convey by presenting those constructs that are important to the person.

In choosing narrative methodology, I also honor my own love for language and its ability to convey the deepest meaning in the most eloquent of ways. Although English is a second language for me, it is also a language I feel most comfortable speaking. I find it is a language of immense variability in terms of vocabulary. I have lived in an Englishspeaking world for the larger part of my life, yet I continue to learn new words to this day. In my eyes, however, no other dialect can compare to the melody of the Ukrainian language. It is my mother tongue; rooted in the Slavic traditions, it has a rich history reaching back as far as the $12^{\text {th }}$ century. It has withstood oppression and diversification, and today, amidst the political turmoil in Ukraine, language is symbolic of the nation's freedom. My own experience with language is important to explicate because for me, culture and language are inextricable concepts. Language, as a communication medium, transmits culture.

\section{The Chosen Methodology}

Narrative study values individuals' verbal accounts of their experiences. Every story, although it can never be a complete account of reality, does preserve elements of greatest relevance to the underlying emergent meanings (Lieblich et al., 1998). At the core of narrative research is the assumption that "there is neither a single, absolute truth in human reality nor one correct reading or interpretation of a text" (Lieblich et al., 1998, 
p. 2). A narrative approach, therefore, advocates pluralism, relativism and subjectivity. As this view is in line with my own theoretical and philosophical perspectives, Lieblich et al.'s (1998) interpretation of narrative research was chosen as the methodological grounding for the study. Using this methodological approach includes accepting the notion that a life story can transmit individual and cultural meaning, since people use building blocks common to their culture when creating their narratives (Lieblich et al., 1998). The stories also reflect the self, or the narrator's identity, as they are subjective (Lieblich et al., 1998). Lieblich et al. warn the researcher to be mindful that the life story provided during an interview is only a snapshot of a single instance representing a dynamic and constantly changing reality. It is, therefore, essential that the researcher involve the participant in the process of interpreting the narratives (Cresswell, 2007). In fulfilling the aim of developing an understanding of how nurses conceptualize CCC in a mono-ethnic LTC practice setting, the lived stories of the participants have been translated into text, and their meaning was analyzed in collaboration between the narrators and the researcher (Lieblich et al., 1998).

\section{Participant Selection}

Participant selection in narrative research is ideally limited to a small number, explains Creswell (2007). Holloway and Freshwater (2007) argue that it is appropriate to recruit between two to four participants for a narrative study. The Thesis Committee and I agreed that recruiting between two to four participants would provide a rich data set that is manageable to analyze within the time limits of the Master's program of study undertaken by the researcher. A total of two participants were recruited for this study, which was the minimum number agreed upon by the Thesis Committee. Although the 
recruitment announcements and posters had continued to be advertised for several weeks after Participant Two had made contact with the researcher, no other interest was generated. As pointed out by Creswell (2007), unless the intent is to develop a collective story, it is appropriate to use between one or two individuals in narrative research. This notion provides further support that the anticipated sample of 2 to 4 participants for this study is appropriate as the purpose of the study is to understand the experiences of individual HCPs specifically with the concept of $\mathrm{CCC}$, rather than to narrate their life stories in full entirety.

\section{Participant Inclusion Criteria}

According to Salkind (2010), inclusion criteria, guided by the objectives of the study, are instrumental in identifying the target sample population and promoting homogeneity of the participants. Homogeneous sampling, according to Holloway and Freshwater (2007), results in the recruitment of participants who share common characteristics that are relevant to the phenomenon under study. Being mindful of the purpose of the study, the inclusion criteria were devised as follows:

1. Regulated nursing professional (RN/RPN)

2. Employed in a Ukrainian-specific LTCH in the GTA for at least 2 years

3. Being of Ukrainian heritage (ideally a member of the fourth wave of immigration)

4. English proficiency, both verbal and written

5. At least rudimentary capacity to communicate in Ukrainian, or better Narrative stories often occur within a specific situation or place, as pointed out by Creswell (2007), thus, it is important for the researcher to be aware of the context of the story. By limiting participant inclusion criteria to those nurses employed in Ukrainian- 
specific LTCHs in the GTA, I had imposed certain contextual constraints on the stories being told. As Hart and Mareno (2013) emphasize, it is important to hear the voices of nurses about their experiences of providing $\mathrm{CCC}$, as the stories of personal experiences may help uncover the challenges and facilitators associated with this individualized process of care. Prior to extending a formal invitation to participate in the study to the individuals who had expressed interest, I first clarified that their professional and personal backgrounds aligned with the selection criteria. The participants' characteristics are described in a later section.

\section{Recruitment Method}

Participant recruitment for the study has been carried out with the help of 6 Ukrainian churches in the GTA. After reflecting on the highly interactive and close-knit nature of the Ukrainian community, I felt it would be appropriate to make the announcement through the churches, which serve as places of community gatherings. Furthermore, most of the Ukrainian-specific LTCHs in the GTA are closely associated with Ukrainian parishes. This increased the likelihood that the announcement would be further spread by word-of-mouth. A conscious decision was made to recruit participants outside of their LTCH workplace to ensure confidentiality (Creswell, 2007). Recruitment posters and bulletin announcements asked those interested in participating to contact me either by a secure, private Ryerson contact phone number specific to the study or by emailing me directly through my Ryerson account, so that their identity was kept confidential from the other members of the congregation, the priest and their employer.

Once the Research Ethnics Board at Ryerson University granted the permission for the study, I approached several Ukrainian parishes in the GTA. I had prepared a 
poster (Appendix A) and a brief text (Appendix B) to be read to the congregation during the announcements. Initially, I called the church office of each parish to establish a contact person; then based on the recommendation of the church secretary, I would contact the individual responsible for the church announcement. In all instances, except for one, this was the reverend of the parish. One of the secretaries that I spoke with explained to me that, according to the parish guidelines, an announcement that does not specifically relate to the functioning of the church cannot be made from the pulpit. However, she agreed to display the recruitment poster on the announcement board. I spoke over the phone with four other priests, all of whom agreed to distribute the announcement. They also graciously blessed my venture. Following our conversation, I either e-mailed or hand-delivered the documents, including the letter for the priest (Appendix C), the poster and the text of the announcement. I also had a private audience with a pastor of one of the congregations in Central Toronto. He too agreed to make the announcement. In total, the announcements, in some form or another, were made in six Ukrainian churches in the GTA.

My intent was to recruit participants through announcements made by the priests at the end of the service, as is customary in the community. As part of the weekly routine, priests make community announcements towards the end of the Sunday service, before the final hymn is sung, to ensure most of the parishioners are in attendance. Announcements include proclamation of recent life milestones of parish members (births, deaths, weddings, graduation/awards, etc.), as well as notice of upcoming community events, fundraisers and holidays. Several of the priests that I spoke with expressed interest in publishing the announcement in the church bulletin. I learned that it is part of 
the standard protocol to read the announcement from the pulpit and include a printed version in the bulletin. After reviewing the Tri-Council Policy Statement 2 (2014), I accepted that, as long as the information published in the bulletin was reflective of the details of the announcement, it would be appropriate to agree to this form of advertisement. The announcements, along with the recruitment poster, were advertised, on average, up to four weeks in each of the six parishes.

\section{Understanding the Participants}

Removing personal identifiers serves to reduce the risk that the readers of a research study will identify the participants. Just as the plot of a novel is anchored in the description of the personages, so is the interpretation of the narrative data fixed in the context of who the participants are and how they interact with the researcher (Lieblich et al., 1998). In the tradition of quantitative research from the positivist perspective, a description of the sample participants is given in the results section (Grove, Burns \& Gray, 2013). With the present study, which is interpretive and qualitative in its focus, the participant sample was small consisting of only two participants; since it is imperative to promote the privacy and confidentiality of these women, and to prevent any potential for their identity to be disclosed, rather than focusing on the demographic description of the participants, such as age, background, and so on, I have chosen to describe some of their general attributes which adds to the understanding of the narrative data.

\section{The Introductions}

The study involved two participants. They both work full-time in two different Ukrainian-specific LTCHs within the GTA, where one of them is employed as a Registered Nurse (RN), the other as a Registered Practical Nurse (RPN). The RN, in her 
late fifties, has been working at the same facility for more than ten years, while the RPN, in her early sixties, has been with her employer close to a decade. Both women immigrated to Canada from Ukraine several years prior to securing employment with their respective LTCHs, and had indicated that they continue to speak primarily Ukrainian in their own homes.

While it was important to safeguard the identities of these women from an ethical perspective, the process of selecting their pseudonyms was a deliberate process, as I chose names that speak to the emotions, feelings and expectations I had towards the thesis work itself. I named Participant One - my 'firstborn' participant - "Nadiya”, which appropriately translates in English to 'Hope'. As a novice researcher, I had lived through a number of anxiety-ridden moments as I developed the thesis proposal, yet the greatest fear of mine was that dark concern of "What if nobody comes forward?". The day that I began to contact the Ukrainian parishes of the GTA asking to aid me in announcing the participant recruitment for the study, I held within me great hope for this study. I hoped that the people of the Ukrainian community would recognize the good intentions of this study. I hoped that there would be nurses - our Ukrainian nurses - who would want to have their voices heard. It was that feeling of hope that kept me buoyant in the next weeks until that first contact with Participant One. To me, she was the human embodiment of all that hope that I had harbored. She was 'Nadiya'.

Having Nadiya with me, I was on my way, it would seem, yet the fear lingered on the surface. What if nobody else came forward? Would I need to modify the participant recruitment process? But deep down, I still had faith. I believed in the value of the research study, and I tried to have steady confidence that there would be others who 
shared this vision. When I was first approached by a woman who, later (and thankfully), became Participant Two, my faith in this endeavor was starting to waiver. It had been several weeks since the announcements were first made and I was beginning to worry that the novelty of the announcements was wearing off. As it turned out, Participant Two was so genuinely interested in the study she took the trouble to locate me in person at Easter mass, as she did not feel comfortable leaving a message on an answering machine. Although I found this admission curious at first, I later came to recognize the eccentricity of Participant Two in her narratives. All that faith I had come into focus in the eyes of this woman. She was 'Vera', my faith.

\section{Data Collection}

\section{Interviews: Text \& Interpretation}

The data was collected via three face-to-face semi-structured interviews, as this is the primary vehicle for data collection in narrative research (Munhall, 2012). Flexibility with questions is inherent to the semi-structured interview approach, which allows the opportunity for better storytelling (Ryan, Coughlan, \& Cronin, 2009). Follow-up interviews are also an essential step of data collection in narrative study, as they give the researcher a chance to check the meanings and interpretations derived against the input of the narrator (Munhall, 2012).

During the data collection period of this study, follow-up interviews were scheduled two to three weeks apart to give me the chance to work through the gathered interview data. A total of six interviews were conducted, three per each of the two participants; two of the interviews, the first and the third, were between 45 to 60 minutes long, while the second interview, the purpose of which was for the researcher to collect 
the disposable camera, lasted 20 to 30 minutes. The interviews were held at locations chosen by the participants, the only stipulation being that the setting is free of excessive interruptions. Among the locations of the interviews were private study rooms at the public library, a meeting room at a community center, coffee shop and a picnic table at a park. The latter two locations were used during the brief second interview, and were deemed to be sufficiently private for the purpose of the interaction. The length of the interviews varied slightly between the two participants; this was decided in response to the level of interviewee's engagement at the time of the interview (Holloway \& Freshwater, 2007). For instance, I had decided to bring Interview One with Participant Two to a close when I observed the participant's attention level began to decline, as was evident through her physical behavior of looking around the coffee shop at the other patrons. At that point in time we had discussed all the questions from the interview guide (Appendix D). At the end of the first interview, both participants were given a gift certificate to a local coffee shop.

With the participants' permission, all interviews were audio-taped using a digital voice recorder and later transcribed verbatim by the researcher. Our greetings and some social small talk were conducted in Ukrainian during the meetings. I had spoken to the participants in English over the phone when we made initial contact. I had wanted to assess the participant's level of comfort with English, as I planned to conduct the interview in English rather than Ukrainian. Translating transcripts into another language results in potential limitations to uncovering the deeper meaning of texts, as it may not always be possible to translate the spoken word directly into another language; thus the decision was made to conduct the interviews in English (Twinn, 1997). Although the 
participants spoke with an accent, it was reminiscent of the style of speaking that I have heard among my parents' peers. It is something I am accustomed to and, thus, I had no difficulty in understanding the participants or in transcribing the interviews. To some extent, however, I did have to make judgments as to assigning the correct tense in some of the text, otherwise the narrative was transcribed verbatim. Such corrections were made to bring clarity to the spoken words, rather than to alter the meaning of the narrative (Mero-Jaffe, 2011).

Prior to the first interview, the participants were given a copy of an informed consent form to read as I reviewed the content with them verbally. The consent form clearly outlined the purpose and description of the study, the requirements of the participants, potential risks and benefits associated with participation, and the overall voluntary nature of participation (Appendix E) (Creswell, 2007; Holloway \& Freshwater, 2007). This document clearly stated the rights and responsibilities of the participants, as suggested by Holloway and Freshwater (2007). Additionally, at all subsequent interviews, I would verbally inquire whether the participants were interested in continuing on with the study. There was no participant attrition during the study.

\section{Photography: The Visual Narrative}

With the purpose of enhancing the verbal narratives of the participants, data was also collected in the form of visual narratives via the medium of photography (Clandinin, 2007). After the first interview, the participants were provided with a disposable camera and asked to photograph images that represented their interpretation of what it means to provide CCC within a Ukrainian-specific LTCH. I had anticipated that the participants might be of immigrant background for whom English was a second language. From 
personal experience, I know that there are instances when it is difficult to find the right words in a foreign language to explain your thinking. Photography has been used in studies of immigration as a tool for capturing the individuals' experiences; for example, McBrien and Day (2012) have used photography to gain critical insight into the experiences of refugee students' while resettling in the United States. Photographs, as captured moments in time, have the capacity to represent the "authentic voice" of the participants and enhance the communication between the researcher and the participant (McBrien \& Day, 2012). Photography as a data collection tool has been used in the human sciences more in recent years; for example, from the sociological standpoint Creef (2004) used photography to study the internment of Japanese Americans during WWII while Bell (2006) used this medium to study the breast cancer experiences of middle aged women.

The participants were given written instructions detailing the goal of and the instructions for completing the activity (Appendix F). Riessman (2008) explains that there are some ethical concerns around photographing people or certain settings. Photographing individuals and institutions may be prohibited to maintain privacy (Riessman, 2008). For these reasons, the written instructions for the photography activity clearly explicated that the participants were not to take photographs of people; instead, they were asked to photograph objects that were representative of the experience from their standpoint. It was also stipulated that the photographs were to be taken outside of the workplace premises to avoid breach of confidentiality and privacy once the photographs are published. I met with the participants approximately one week prior to our third, and final, interview to collect the disposable cameras. I then developed two sets 
of photographs, one of them for the participants' personal archives and the other for use in relation to the publication and presentation of the study findings. Both participants chose to keep their respective set of photographs.

\section{Protecting the Participants: A Researcher's Duty}

Creswell (2007) gives some general suggestions for storing of collected data, namely developing backup copies of computer files, using high-quality tapes for audiorecording of interviews, developing a master list of the types of information that has been gathered, protecting the anonymity of participants by removing their identifying information from the data, and developing a data collection matrix to assist in visually locating and identifying information for the study. I adhered to these procedures throughout the study. The hardcopies of interview transcripts have been kept in a locked filing cabinet in my home office, while electronic data stored on a password-protected USB flash drive also kept under lock-and-key when not in use. The interviews were recorded using a dictation app on the researcher's password protected smart phone. The recordings were then transferred to the USB flash drive immediately following the interview, and the original recordings were deleted from the phone. In the Introductions section, I detailed the process of choosing pseudonyms for the study participants; this was done with the aim of protecting their right to privacy and confidentiality.

\section{Data Analysis: The What \& The How}

Narrative data may be analyzed in a variety of ways, including content, structure, style of speech, motives, attitudes and beliefs, and the cognitive level of the narrator (Creswell, 2007). The approach the researcher chooses for data analysis, to a large extent, depends on the predominant interests of the researcher - if the researcher is interested in 
what happened and why, the content of the narrative is for consideration (Clandinin \& Connelly, 2000). Meanwhile, the narrative form demands attention when the researcher is concerned with the way the story is pulled together by the narrator, including the use of metaphors, syntax, and allegories (Freshwater \& Holloway, 2007). Lieblich et al. (1998) confirm that both content and form, meaning what is being told and how it is being told, must be considered when analyzing narrative data.

Lieblich and colleagues (1998) propose four classifications of narrative data analysis, including holistic-content, categorical-content, holistic-form, and categoricalform. A holistic-content approach focuses on establishing the connection between emerging theme categories to illuminate the narrator's whole story, typically within the sequence the participants experienced in real life (Lieblich et al., 1998). Meanwhile, the categorical-content approach considers the individual components of the narrative to identify emerging themes that reflect the specific phenomenon as opposed to an entire story over the participants' lifetime (Lieblich et al., 1998). When considering the form of the narrative, Lieblich et al. differentiate between holistic-form and categorical-form approaches, in that the first is centered around the plot analysis of the entire narrative while the latter conducts plot analysis only on those parts of the narrative that are directly related to the research question.

In the case of this study, both holistic-content and holistic-form approaches were discarded, as they did not address the purpose of this study. Meanwhile, categoricalcontent analysis was identified as highly relevant to the study purpose of narrating the participants' experiences with CCC; additionally, the categorical-form analysis approach was adapted to better align with the available data. Since Lieblich and colleagues (1998) 
believe that narrative form contributes to the interpretation and meaning of the narrative, the participants' photographic data were subjected to categorical-form analysis. This was an intentional decision so that the meaning of categorical-content data emergent from the analysis of interview texts would be enriched. Both the categorical-content analysis and the visual analysis of the photographs as part of the categorical-form analysis represent the first level of analysis, or the emergent theme analysis. Following this, the data was further subjected to two increasingly more abstract levels of analysis. First, a comparative theme analysis was completed to collate the elements inferred from the participants' narratives during the emergent theme analysis. Then, the data were integrated via metaphoric representative analysis, resulting in a graphic representation of the major themes of the study.

The findings viewed through the first two levels of analysis, namely emergent theme analysis and comparative theme analysis, are presented in Chapter 4: Findings, while the metaphoric representative analysis outcomes are part of the discussion in Chapter 5. The presented findings are supported by direct quotations from the participants, however, my voice as the researcher appears alongside the findings. This is in keeping with the narrative methodology as described by Lieblich et al. (1998), who believe that the emergent understanding of the study findings is co-created by the participants and the researcher. It would be counter to Lieblich et al.'s methodological approach for me as a researcher to extract myself from the Findings chapter. Creswell (2013) writes that a researcher conducting narrative study may discuss his/her "own situated beliefs" alongside the narrative findings to aid in revealing additional features of the experiences of the participants. My knowledge and understanding has been informed 
through my own experiences with culture and professional experiences as a nurse, as well as through my academic background and engagement with the literature. My reflections, however, are directed at the data within the participants' narratives. I had completed member checking with the participants during the third interview to determine whether my interpretation of the data aligns with the meaning intended in the narratives (Creswell, 2013). The thought processes supporting the analysis decisions are presented below.

\section{Categorical-Content Analysis: Interpreting The What}

The data analysis of this study was guided by the research question aiming to understand how two frontline healthcare nurses at mono-culture LTCHs conceptualize and deliver CCC. The population of interest, as has been mentioned, was nurses of Ukrainian heritage, either RNs or RPNs, working in Ukrainian-specific LTCH in the GTA. As explained earlier, Lieblich and colleagues (1998) emphasize the value in understanding what has been said, as well as how it was said. Rather than focusing on the narrator's whole life story, I was primarily interested in her experiences with CCC. The what portions of my participants' narratives, therefore, were most appropriately interpreted through the lens of the categorical-content analysis approach proposed by Lieblich et al., which is akin to the open coding procedure used in qualitative descriptive studies. It is similar to the traditional content analysis and thematic analysis whereby smaller sections of the written text are isolated and are grouped according to themes into categories (Holloway \& Freshwater, 2007). The main focus of the categorical-content analysis is on the narrator's interpretation of what had transpired, why it occurred and who was involved (Lieblich et al., 1998). The elements arising through the categorical- 
content approach are representative of the first level of analysis, or the emergent theme analysis.

\section{Categorical-Content Analysis Steps}

Lieblich and colleagues (1998) introduce the steps of the categorical-content analysis process. These steps were applied as follows:

1. Text is Selected for Analysis: Researcher is to read and re-read the interview transcripts until she develops a familiarity with the content. The transcribed interviews were re-read upwards of twenty times; during some of the readings, I would follow along to the recording of the interview, noting the emotions and intonations that were present. Since I was only interested in soliciting descriptions of those experiences related to instances of CCC provision, rather than the narrative of the whole life story, I was able to consider all of the obtained text for content analysis (Lieblich et al., 1998).

2. Content Categories are Defined and Subtext is Sorted into Categories: Lieblich and colleagues (1998) note that the titles of the categories can be selected in one of two ways, either using those pre-defined through a theory, or via exhaustive rereading of the collected text for emerging categories. The latter approach was used in order to capture the emergent meaning of the narrative rather than attempting to forcefully fit text into pre-determined categories. Individual words, sentences or expressions were retrieved from the narratives and grouped under general headings reflective of their content. For example, initially, all mentions of language or communication were collected under the heading of Linguistic Congruency. The names of the categories would emerge with time from the ideas 
that cut across the text, rather than being imported from related theories or literature. Lieblich et al. warn that this may be difficult to accomplish, since the researcher may approach the data holding some theoretical commonsense and understanding, however, I made a conscious effort to develop categories based on the narrative data rather than theoretical constructs evident in the literature. For example, in the narratives of Participant One, all comments, words or statements about the LTCHs, including the focus on the cultural environment of the facilities, were initially grouped under the heading Home. Once the data were sorted, it became clear that the Ukrainian feel of the home was the more significant element associated with CCC. The title of the category was therefore changed to a direct quote from Participant One "It's a very Ukrainian environment" that confirmed the importance of the Ukrainian-specific cultural elements present within the facility that made CCC possible. The identification of the categories and the subsequent assigning of subtext to them is a circular process, explain Lieblich et al., whereby the researcher continuously re-reads the text, suggests and refines the categories, and sorts the subtext which fits into these categories. Some refinement of categories occurred throughout, which, as Lieblich et al. explain, is expected. There is no set number of categories that a researcher is expected to derive; the yardstick to adhere to is that a balance of categories must exist so that the richness of the subtext is preserved (Lieblich et al., 1998). Further to this, category definitions and content sorting were verified in discussion with the Thesis Supervisor, as Lieblich et al. (1998) suggest that feedback from other scholars is warranted for this process. 
3. Conclusions are Drawn. The final step of data analysis is to put forward conclusions based on the attained data, and to present them descriptively as findings (Lieblich et al., 1998). Analyzing the individual categories, it became apparent that no cluster of ideas was sufficiently broad to explain how CCC is conceptualized. Instead, the conceptualization of CCC was evident under one umbrella term, with the remaining categories acting as the supporting spokes of the umbrella. In both narratives there were elements that either facilitated or complicated the nurses' ability to provide CCC. Thus, the various categories identified in step 2 were then descriptively analyzed based upon the comments I added in the margins of each transcript during line-by-line coding throughout the reading and re-reading analysis process. It was this continuous re-reading of the data, however, that served as the basis for the comparative theme analysis, or the second level of analysis, as I began to draw parallels between the elements of the participants' narratives coming into focus. To verify my understanding, I sought the participants' input during each of our final interviews. We engaged in dialogue to further the understanding of the themes that had emerged, and only minor shifts to my interpretations were suggested by the participants.

\section{Analyzing the How: Round Hole, Square Peg}

It would be logical to assume, given my initial use of Lieblich et al.'s (1998) methodology, that I would choose the same process to analyze the form of the data, or the how it was said as part of the first level of analysis. Lieblich et al. write that the form can also be analyzed either categorically or holistically. While different in the quantity of narrative data they consider, both categorical-form and holistic-form analysis focus on 
the linguistic features of the narrative, the words and expressions one uses in their narrative to convey feelings, thoughts and emotions (Lieblich et al., 1998). As I began to focus on the emotions and attitudes conveyed by the participants, it became apparent to me that a significant form of expression employed by the participants was the use of photographs, which, in some instances, would convey the narrator's message in a more detailed manner than the verbal account. This was especially apparent when the participants would use words almost as place holders when they had difficulty expressing their thoughts in English. Additionally, in their narratives there was varied use of grammar, as well as altered pronunciation, reflective of the Eastern European origin of both participants. After reviewing the intent behind Lieblich et al.'s (1998) process of dissecting paralinguistic features of the spoken words as part of categorical-form analysis, I came to realize that the ethical-moral dilemma I was facing could be resolved if I considered other approaches for uncovering the experience of CCC with the participants other than using the spoken word. Upon further engagement with the data, I came to appreciate that the deeper understanding of verbal expressions were enhanced by the photographic data as a means of conveying the participants' thoughts, feelings and emotions about the concept of interest. The participants had the opportunity to take photographs of images that were symbolic of their understanding of CCC. The use of visual data was a conscious decision as I explained earlier, based on the expectation that the participants of the study may be of immigrant background themselves. The photography activity intended to give the participants another medium to express themselves in instances where it was difficult for them to find words to reflect their feelings, thoughts or impressions (Keats, 2011). Consequently, at times, the metaphors 
reflected in the photographs spoke volumes, while the voices of the participants were mere whispers. As I re-read the transcripts, following along to the audio-recordings, I noticed how the participants' references to the photographs added depth and further clarity to the emerging understandings. I decided to consider the metaphors within the photographs as a way of elaborating on the what of the narratives. The photographs themselves became the how.

\section{Imagery \& Research}

The culture of the human race has been depicted through signs, symbols and images since ancient times. For example, some of the oldest cave paintings date back 35 000 years, yet archeologists continue to study their meaning to this day (Bubar, 2014). Visual assessment tools, such as the FACES Pain Scale, that are used in modern medicine to understand the patient's experience of pain further attest to the validity of images as representations of contextual and individualized understanding. Visual representation is also extensively used for research and includes paintings, videos and photography (Riessman, 2008). Imagery is particularly relevant in qualitative research, including narrative studies (Keats, 2009).

Narrative studies, true to their aim, consider visual images, such as photographs, for their interpretive quality. Early use of imagery in human sciences research was concerned with capturing realistic portrayals of social inequalities (i.e. child labor); the intent was to 'document' the phenomenon (Reissman, 2008). The photographs in these earlier human research studies used to be taken by researchers and professionals. Narrative researchers, on the other hand, release the control of the camera to the participants, who then choose what images to photograph (Reissman, 2008). In narrative 
studies, it is understood that the captured images are chosen by the participant for a specific reason. The participant-photographer and the researcher then collaborate on narrating the captured image (Reissman, 2008). It is understood that the "seeable", explains Reissman, can go deep beyond the "sayable". The captured images, however, need to be contextualized through words; this process of visual analysis is guided by the research question.

\section{Photography as Emotional Expression}

Lieblich and colleagues (1998) conclude that it is a challenge for a narrative researcher to predict the exact data analysis process for the proposed study before the actual data has been collected. This idea of evolving the data analysis of narrative studies has been considered by other researchers, including Nafsin Nizum (2015). In her study of the experience of resilience in South Asian immigrant older adults living with mild dementia, Nizum describes her own journey of selecting a narrative approach that would reflect and respect the narratives of her study participants who were in the early stages of dementia. The emergent view of the data should be more fluid, reasoned Nizum, informed by the kind of data that has been collected. This view resonated with me, as the verbal narratives of my participants seemed to be incomplete without incorporating the photographs they had taken. Considering the messages within the photographs expanded my capacity to build on the first level of analysis of the findings, which led to the subsequent comparative and metaphoric analyses.

In their writings, Lieblich and colleagues (1998) open the door to other ways of collecting the narrative. The chosen approaches should work in concert with each other in order to blur the line between the text, its reading and interpretation. As a researcher, I 
came to realize that I could be innovative by supporting the written text of the participants' narratives with photographic images. In choosing this approach I further promoted the theoretical backbone of social constructivism upon which this study was built. In addition, the notion that pluralism enriches our understanding gives support to the use of photos to represent meaning and emotion (Lieblich et al., 1998).

We live in the visual world that stimulates our senses. I believe that the photographic images can contribute to the emergence of the narrative meaning. Photography is a genre of aesthetic representation of experience. Through the imagery of the photograph, that which was felt by the individual becomes a "shared experience" with the viewer (Riessman, 2008). Lieblich et al. (1998) conclude that the structure of narrative text can be analyzed through empathetic and sensitive reading to gain insight into the emotional experiences of the participant, since the narrative of a speaker can be emotionally charged. As part of the modified categorical-form analysis, I therefore explored how the spoken word and the photographic images intersected to reveal the narrative thread. For the content analysis, conclusions were drawn on the empathetic and sensitive reading of the linguistic features and sensitive review of photographic images, as suggested by Lieblich et al. As I began to explore the potential approaches for analyzing the photographic images as representing the structure of the narrative data, I made reference to the work of Reissman (2008). In her book Narrative Methods for the Human Sciences, Reissman introduces four approaches for analyzing narrative data, including visual analysis. In the following section, I describe how I adapted Reissman's work to analyze the photographic images taken by the participants of this study. 
Visual Analysis: Interface of Word and Image. Visual analysis integrates the written words with images to uncover the ways through which individuals perceive the surrounding world (Clandinin, 2007). Visual imagery, therefore, is a form of storytelling. In reflecting on the visual depiction of their experiences and understanding of the phenomenon, the participants may further express feelings, thoughts, emotions or ideas that were not verbalized at the initial interview, yet have been prompted by the research questions. When a researcher collaborates with the participant to understand the message of the photograph, she asks how and why the participant-photographer took the particular shot. The details of the images in the photographs - such as lighting and positioning can convey the mood or emotions of the message within the image, or of the imagemaker themselves (Reissman, 2008). When completing visual analysis, the participantresearcher dyad needs to consider how the image was produced (i.e. was the image found "as is" or was it "made" by the photographer), the details and the meaning of the image itself and how it might be "read" by the audience (Reissman, 2008). Sometimes, the intent of an image captured by the participant is not to reveal, but rather to conceal an idea (Reissman, 2008). Understanding of this subtext may emerge through the collaborative narrative between the participant and the researcher.

Clandinin (2007) writes that visual narrative inquiry "is an intentional, reflective, active human process in which researchers and participants explore and make meaning of experience both visually and narrative" (p. 280). To this extent, I, as the researcher, had the obligation to engage the participant to narrate the photographs themselves. Prior to analyzing the photographs with the participants, however, I had journaled my own thoughts, feelings and understandings about the images, reflecting on what had been 
discussed in the initial interview with the participant. Clandinin (2007) encourages the researcher to write down possible meanings of the photographs first, before discussing them with the participants. Reissman (2008) writes that the images must be continually interpreted as new data are collected to uncover the shifts in understanding. In reading the images, I would refer back to the philosophical position, the theory, the literature review and the research question. Still, multiple readings of the same image are possible; there is never a single "correct" reading, explains Reissman (2008). Just like the text of the narrative is bound by time, history and place, so are the visual images.

During our third interview, guided by the suggestions in Reissman's (2008) work on visual analysis, I asked both participants to share with me the message that they intended to convey through their photographs. We also discussed how the participants decided what to photograph, as well as their experience of using the camera as a tool for data collection. Together, through dialogue, we worked to connect the photographs to the themes emerging from the participants' narratives. For a list of questions related to the photography activity, please refer to Appendices G and H, or Second Interview Guide: Camera Collection and Interview Guide: Third Interview, respectively.

\section{Preserving the Message}

The photographs included in this work are in their original form. Reissman (2008) acknowledges that the technological advancements of present day (i.e. Photoshop software) may be used to alter images. I felt that by altering the photographs in any significant manner (i.e. adjusting the colors, sharpness or contrast), I would be disregarding the meaning of the message that the participants intended to convey through the photographs. Furthermore, edits such as cropping the image might significantly alter 
the meaning of the photograph by removing an essential representation of the participant's experience. Such interventions could alter or distort the meaning of the nurse's experience of providing CCC. The participants had been in control of the camera and had chosen the specific images with intent of conveying a deeper meaning. I demonstrated respect for their identity as a CCN by maintaining the integrity of the photographs.

\section{Higher Level Analyses}

As was mentioned at the onset of this chapter, the narrative data was subjected to three levels of analysis. The understandings emerging from the hybrid of categoricalcontent and visual analyses represent the first level of analysis. As part of the second level of comparative analysis, I had explored the participants' personalities and their influence on the narratives. It could be argued that the degree of judgment and bias in my conclusions about the personalities of the participants could be significant. I only had a few interactions with the participants, limited in time and scope, so the personality traits that I deduce may not be truly reflective of the participants as individuals and as nursing professionals. I, therefore, exercise caution in using descriptions of absolute value, and instead I focus on the personality traits, emotional expressions and linguistic nuances that came through in our moments of dialogue.

Lieblich et al. (1998) refer continuously to the use of imagination, acknowledging that the narrative includes typologies or the areas for the interpretation. While researchers are doing the description of the findings, they are also continually looking for generalizations that can be justified based on the feelings and memories that are evoked in the researcher (Lieblich et al., 1998). One needs to be careful about pulling apart those 
inferences that can be qualified from findings and those that need to be discarded, because they are assumptions, and, as such, they are faulty. Lieblich et al. reinforce the notion that the researcher is always bringing assumptions to the dialogue and analysis of the narrative, and therefore, needs to continuously reflect back on the data, looking for instances where information may be qualified by the facial expressions, quotes or behaviors noted within the participant. This is of particular relevance, according to Lieblich et al., during categorical-form analysis, where the use of language, facial expressions and body gestures, pitch and tone of the voice, are all elements that both add to and constitute data. Such an approach may not be acceptable from the positivist perspective, where it can be skewed as biased, judgmental and interpretive, since it holds a clear distinction between the experiential data and personal inference (Gray, 2009). Lieblich et al., however, hold that the inference of personality of the participants is, in fact, part of the findings.

The researcher's inferences about the personality of the participants are relevant for the relationship between the researcher and the participants, explain Lieblich et al. (1998). The colleagues also emphasize that the participants' understanding of their own values and beliefs may emerge through their experience of sharing the stories with the researcher. The interactions between the researcher and the participants, as well as the personalities of both parties, according to Lieblich et al., create context for the narrative. It is this understanding that further supports my decision to include my own voice in the Findings chapter. As a researcher, I am honoring the style and the personality of the participants in the inferences of their narratives by extracting the new understanding directly out of the narrative data, and supporting it through the observations of their 
personalities. Lieblich et al. (1998) explain that a degree of relational context is critical to the understanding of the narrative data. By including examples of interactions between myself and the participants that are exemplary of their personality traits, I share with the reader of the study an idea of who the narrators are as individuals and professionals. This way, the reader comes to recognize that there is a person behind the written text.

Finally, the third level of analysis is the metaphorical representation that appears as a graphic alongside the discussion in Chapter 5. As the highest level of analysis, it is largely abstract and symbolic in its representation. Further detail about metaphorical analysis is presented in the next section on reflexivity.

\section{Reflexivity}

Prior to entering the data collection period of the study, the researcher needs to understand what influence she might have on the study outcomes (Streubert \& Carpenter, 2011). This type of self-reflection in qualitative study is called reflexivity (Streubert \& Carpenter, 2011). Elliott (2006) explains that reflexivity is the analytical exploration and discussion of the researcher's own biographical and theoretical perspectives, and how these impact the relationship with the study participants, as well as the interpretation and analysis of the collected evidence.

As an active member of the Ukrainian community in Toronto, a first generation Ukrainian-Canadian, and a Canadian-educated nurse, I have personal and professional understanding of the interaction between CCC and healthcare. Earlier in this work I have explicated my philosophical assumptions, as well as my personal and professional experiences, all of which have shaped my understanding of what it means to provide CCC. By acknowledging that my own stories, beliefs and experiences may impact on the 
course of the study I would strive to develop a heightened awareness of self as acting in the social world through active journaling (Elliott, 2006). As my self-awareness deepened alongside my understanding of the research phenomenon, I continued to reflect on my personal biases, assumptions and prejudices through journaling. This aided me in separating my own biased interpretations from the intended meaning of the participants' narratives. I also noted the degree of overlap between the worldviews I hold versus those of the participants. I would then discuss my interpretations with the participants during our interviews. Giving transparency to my own background, interests and ideas helped promote my trustworthiness as a researcher (Holloway and Freshwater, 2007).

\section{The Metaphorical Analysis: Creating a Graphic}

A creative embodiment of the reflective process is presented in this written work in Chapter 5: Discussion in a form of a graphic that integrates the major themes that became evident during the third level of analysis, the metaphorical analysis. In creating the graphic, I had relied on the knowledge of my Ukrainian ancestry, incorporating traditional elements and style of architecture into a drawing of a house. All other elements are symbolic of the participants' narratives. Initially the graphic was created in black and white, however, in keeping with the style of color photography that was included in Chapter 4: Findings, I chose to add color to the model. This may also help the audience recognize the Ukrainian elements featured, such as the guilder rose. A more detailed description of the graphic can be found in Chapter 5.

In creating the graphic I had relied on modern technology to present a crisp, colorful image. The image was created using a Sony Sketch application on a Samsung Galaxy Tab. This application offers a broad color palate. Additionally, it has a zoom 
feature, which aids in editing the smaller details. A TARGUS stylus was used as the drawing tool. The thick tip of the stylus was ideal for the broad strokes; however, it was a bit of a challenge to create precise details.

\section{Rigor of Narrative Research}

Following the use of Lieblich et al.'s (1998) approach to data analysis, it was logical to also apply their criteria for evaluating rigor in narrative research for the study. The four criteria of width, coherence, insightfulness and parsimony are specific to categorical-content analysis, and are described below.

1. Width refers to the comprehensiveness of evidence (Lieblich et al., 1998). The colleagues believe that quality of evidence can be bolstered through use of direct quotations from the participants. Using the voice of the subject of the story being told is intended to capture the "full" story of the lived experience (Campbell \& Machado, 2013). I also promoted quality of the data by reviewing the interview transcriptions, data interpretations and emerging themes with the participants for accuracy (Holloway \& Freshwater, 2007).

2. Coherence, the second criteria of rigor in narrative research according to Lieblich et al. (1998), refers to the ability of the individual parts of the narrative to represent a meaningful whole. The use of visual narrative was important for fulfilling the coherence criteria, as the photographs were instrumental in bridging the spoken words with the literature. Furthermore, the three level of analyses that were carried out with the data sets would build upon each other. The final outcome of the third level of analysis, the 
metaphorical analysis, is a graphic that incorporates the major themes emerging from the participants' narratives.

3. The use of photographs is representative of Lieblich et al.'s third criteria for rigor, namely insightfulness. Insightfulness is the sense of innovation or originality in the presentation of the story and its analysis (Lieblich et al., 1998). Supplementing the written word with the visual imagery of the photographs added new dimensions for the interpretation of the participants' narratives.

4. Finally, the fourth criterion of rigor in narrative study as presented by Lieblich et al. is parsimony, which is the 'closeness' between the presented interpretation and the narrator's account, or how well the written interpretations actually represent the narrator's story. Several steps were taken to promote parsimony during this study. Firstly, the interviews were audiotaped and transcribed verbatim to ensure that all collected data is preserved. Secondly, follow-up interviews were conducted as an opportunity to seek clarifications from the participants and to confirm with them the interpretations of the themes lifted from the text. Clandinin and Connelly (2000) similarly favor the collaboration between participants and researcher in negotiating the meaning of the stories as a way of validating the data analysis. Lastly, engaging in reflexivity by keeping field notes helped separate my personal interpretations from the voiced stories, so that the presented findings are a true representation of the narrator's intent. The challenge of narrative research is to collect individual stories and to shape them into a collective 
narrative that gives meaning to the experienced phenomenon (Creswell, 2007). The researcher's narrative may itself become reflected in the collected data, and so it is imperative that the researcher keeps in mind the primary intent of the study is to produce a narrative that is reflective of the narrators' experiences.

\section{Ambiguity of the Narrative Approach}

Narrative research commonly generates a significant volume of data (Lieblich et al., 1998). Narrative research, however, is not guided by a prescribed hypothesis; rather, a research question based on some prior understanding of a phenomenon gives direction to the process of the study, including the participant selection and data collection methods (Lieblich et al., 1998). The direction of the study begins to emerge in concordance with data collection. The analysis of narrative data is an interpretive process, and, as such, it "is always personal, partial, and dynamic", explain Lieblich and colleagues (p. 10). Lieblich et al. warn that this ambiguity associated with narrative research may not fit the thought process of all researchers, therefore, it should be undertaken by those whose worldview tolerates the shifting nature of interpretive conclusions. When interacting with the participant, the researcher must be sensitive to the narrator's voice and the meanings of what is shared; the interpretive findings, though, need to be based in justification (Lieblich et al., 1998). Since most narrative research does not lend itself to replication, the researcher should continuously verify the interpretations against the narrative text (Lieblich et al., 1998). The presented findings are then supported by the narrative text and the literature related to the phenomenon of interest. As such, I ensured that interpretations were verified by reading and re-reading the text, as well as by having conversations with 
the participants in the third interview that would allow me to confirm that my interpretations adequately reflected the participants' narratives.

\section{Ethical Considerations}

Ethical issues may arise during any stage of qualitative study, warns Creswell (2007). As the proposed study had intended to use human subjects, an application was submitted to the Research Ethnics Board (REB) at Ryerson University, where the researcher is enrolled in the Master of Nursing graduate program. An ethics certificate was provided for this study (Appendix I). As mentioned earlier, the participant recruitment commenced only upon receipt of written permission from the REB. Secondly, informed consent was collected from the participants enrolled in the study. As part of the informed consent, the participants were provided with a detailed description of the study and its goals, what expectations are associated with their agreement to participate in the study, guidelines around their privacy and confidentiality, and the researcher's intent to avoid placing any undue risk on the participant (Creswell, 2007; Holloway \& Freshwater, 2007). Additionally, the consent form emphasized that participation in the study was voluntary, thus the participants were free to withdraw from the study at any point without repercussions (Creswell, 2007).

A gift certificate to a local coffee shop in the amount of $\$ 10$ CAD was presented to the participants at the end of the first interview, as a means of demonstrating acknowledgement of the time invested. The amount gifted was not intended to act as a financial incentive for participation. Furthermore, I believe that this amount falls within a reasonable budget for the principal investigator and is not too great of a value to have been perceived by the participants as a coercive incentive. As is mentioned in Tri-Council 
Policy Statement 2 (2014), to promote the voluntary nature of the participation, the offered incentive "should not be so large or attractive as to encourage reckless disregard of risks" (p. 29). It should be noted that both participants had initially tried to refuse the gift card, expressing that their interest in the study was not motivated by the promise of a gift card. With reassurance from me, they ultimately did take the cards offered, understanding that it was a symbol of our mutual appreciation and time commitment to each other. By following through on the promise of the gift card I was able to promote trust within our interactions.

As a researcher, I vowed to promote privacy and confidentiality of the participants prior to, throughout and post-study completion. This was accomplished in a number of ways. The collected data is being stored on a password-protected USB flashdrive, with the hard copies of the data being locked away in a filing cabinet. Personal identifiers, including names, were removed from the transcripts, wherein the participants are referred to by pseudonyms (Creswell, 2007); the participants' personal data, collected through use of a demographic form (Appendix J) is only known to the researcher and the Thesis Committee. Whenever referring to the study participants outside of my interaction with the Thesis Supervisor and the Thesis Committee, I refer to the participants by their pseudonyms. The researcher must conceal the identity of participants so that they are not identifiable in publications or presentations associated with the research study, emphasizes Clandinin (2007). While the participants would choose the location for our interviews, I encouraged them to select places that allow for private conversation. In light of the tight social bonds that exist within the Ukrainian community in GTA, there is potential for participant identities to be exposed. To minimize this risk of exposure, no 
single LTCH was approached during the participant recruitment. Rather, the participants were recruited through announcements and bulletins at the Ukrainian churches in the GTA, as I explain earlier. The names of the participants' employers have also been removed from text.

\section{Photography and Ethics}

Since use of visual data is a fairly recent phenomenon, there were some ethical concerns that needed to be considered with respect to use of this form of data. Woodhouse (2012) writes that the participants might not like some of the images they have photographed or they might question whether those images truly reflect their experiences. I encountered the earlier issue with Participant One, when some of the photographs she had taken did turned out to be of poor quality, while some others were not even captured on film. As was explained to me by the processing lab, the photographs were taken from too close of a distance, which significantly reduced the amount of lighting. As the equipment used for the activity was a disposable camera, there were certain limitations that are inherent to this device. For optimal results, photographs are to be taken at least four feet away from the object and in broad daylight.

Participant One had attempted to capture images indoors from up close. I gave the participant the opportunity to express her feelings about the issue, however, it quickly became apparent through dialogue that the participant's overwhelming worry was that there would not be enough visual data to support her narrative. I then reoriented the participant's attention to the photographs that did turn out. Through some gentle encouragement, we were able to shift the focus of our discussion to the meaning of the available photographs, rather than focusing on the shortcomings of technology. 
Collaborating with the participant on contextualizing the images helped the participant find the meaning in the available images.

Another ethical concern around use of photographs produced by the participants is the issue of copyright, especially with respect to reproducing the images in print (Woodhouse, 2012). During interview three, I gave each of the participants a copy of the photographs and they had control of the images throughout our conversations. I refrained from asking questions about a specific photograph from the set. Instead, I asked general questions and then made note of which photographs the participants would refer to in their answers. I then sought the participants' verbal permission to use the photographs in my work, referring specifically to those photographs around which the narrative was built. Both participants gave me verbal permission to use their photographs. Additionally, the Information and Consent Form that the participants signed prior to start of the study included a statement, by signing, they agreed to have their photographs used for presentation and publication purposes, and that their identity will always be kept confidential.

\section{The Power Play}

The potential power imbalance between the researcher and the participant is also an area for ethical concern, particularly during a highly interpersonal research like narrative study. For example, if the researcher is in possession of the interview guide, $\mathrm{s} / \mathrm{he}$ is in control of the process. Holloway and Freshwater (2007) believe that the power imbalance can be lessened when the researcher resists the desire to speak during the interview, consequently suppressing his/her subjectivity. While the researcher might have a strong grasp of the multidimensionality of the phenomenon of interest due to the 
extensive experience with it, as well as knowledge gained by engaging with the literature, it is also possible that the researcher's conclusions are flawed, having been formulated through inconclusive research or based in anecdotal evidence stemming from practice. The likelihood of erroneous conclusion based on preconceived notion can be minimized through reflexivity and an audit trail (Munhall, 2012).

Journaling my experience, thoughts and interpretations was instrumental to my ability to reduce the power imbalance between myself and the participants. At the onset, I recognized the importance of giving the participants a choice as a way of releasing some of that power that I hold as a researcher. I relied on both verbal cues and non-verbal behavior to do this. For instance, whenever it was appropriate, I shared my own insight and understanding of our Ukrainian culture, as a means of validating the participants' expressed positions. As both participants became more comfortable with me, they began to ask more questions about the study, as well as inquire into my personal background. In order to build a relationship with the participant, I would use my own personal and professional judgment in deciding how much information I would disclose about myself. Given my personal and professional presence on the World Wide Web, along with my involvement in the Ukrainian community, it would be reasonable to deduce that either of my participants could have collected external information about me. To this extent, I was honest in my answers with the participants whenever they inquired about my background, but I would direct the conversation back to the research topic whenever it was needed.

I was also deliberate in releasing some of the control to the participants through my non-verbal behavior. For example, I made an effort to always arrive a few minutes prior to start time for all the interviews. My intent in doing so was to give myself the 
opportunity to find a spot at the meeting location that would allow the optimal privacy. In choosing my own seat, I took the chair that would give the participant the chair that would ensure the opportunity to leave without interruptions should she choose to terminate the meeting. Giving the participant the freedom to leave without interruption was a way of respecting her decisions.

\section{Understanding My Role}

Parker (2005) urges the narrative researcher to remember his/her role is not that of a therapist, therefore, discouraging the notion of narrative interview being a therapeutic endeavor. Instead, Parker (2005) and Mishler (1986) acknowledge that verbalizing a story about experience may act as a catalyst for change for the narrator (in this case - the participant). Lieblich (1996) also acknowledges the potential dual role of narrative study as both research and therapy. Still, the interviewer is to remain aware of the potential that the participants may not present the entirety of their personal experience with the phenomenon, choosing to disclose only those segments they judge to be socially acceptable (Lambert \& Loiselle, 2008). Streubert and Carpenter (2011) note that the likelihood of obtaining socially acceptable responses may be diminished through the establishment of a positive and trusting rapport between the researcher and the narrator. I was cautious to demonstrate an appropriate level of understanding towards the participants, avoiding extreme expressions of emotion. For example, when the participants shared the negative experiences of providing $\mathrm{CCC}$, I encouraged them to complete their recollection without interrupting them with follow-up questions. Streubert and Carpenter also suggest assuring participants that their confidentiality will be protected in all aspects of the study, as this is an important step in the building of trust 
between the researcher and the narrator. I did this consistently throughout the interviews with both participants.

As a researcher, I was also concerned that I may potentially encounter narratives that exhibit poor care practices, such as staff abuse or neglect of residents. Such instances could jeopardize my ethical duty of maintaining participants' confidentiality, as noted in the Tri-Council Policy Statement 2 (2014), as I would be obligated by ethical, legal and professional requirements to report these instances to appropriate authorities to protect the health, life or safety of the study participants or a third party. As part of my personal preparation for the commencement of the study, I reviewed the professional code of ethics, including the 2008 Centennial Edition of the Code of Ethics for Registered Nurses published by the Canadian Nurses Association. As recommend by the Tri-Council Policy Statement 2 (2014), I planned to consult with a colleague - namely the Thesis Supervisor - should I encounter instances where there was a conflict between the law and principles of ethics to ensure that I take appropriate course of action. Further to this, despite any aversion, personal or professional, that I might feel towards the narrative accounts of abuse or neglect, it was my intent to refrain from imposing false conclusions that would distort or cover-up the true meaning of these narratives (Ayres \& Poirier, 2003). I did not detect any moments of unethical behavior in the narratives of the participants.

\section{Concluding Remarks}

This chapter outlines the qualitative research approach chosen for the proposed study, including details of the data collection, as well as the three levels of analysis completed to uncover the storied meaning of the narrative data. Justification for the use of narrative study is provided, stemming from the researcher's theoretical lens of social 
constructivism and the nursing profession's affinity for storytelling. The reader is also introduced to the two study participants. Four criteria for evaluating rigor of narrative research are presented, along explanations how these were maintained throughout the study. Finally, the potential ethical concerns of informed consent, protection of privacy and confidentiality of the participants, and the researcher-narrator power imbalance were considered. Ethics surrounding use of photography as a data collection medium were also discussed. 


\section{CHAPTER 4}

\section{Findings}

Over the course of the data collection period, I have been exploring the concept of CCC as it relates to Ukrainian-specific LTCHs through interviews with two nurses of Ukrainian heritage. In this chapter, I present to the reader a description of the findings as extracted from the data transcripts through a first level analysis, categorical-content analysis. This is further supplemented by interpretations emerging from visual analysis of the photographic data. I first begin with Nadiya's voice, then presenting Vera's. I conclude this chapter with a comparison of the two sets of narrative data. At that point, the narratives are subjected to a higher level of comparative analysis that facilitates the emergence of common attributes and elements of CCC.

\section{Nadiya - Надія}

Throughout her narrative, Nadiya describes four distinct, yet inter-related elements that are integral to her ability to provide CCC. These include a) attributes of a culturally competent nurse (CCN), b) nurse's cultural wisdom, c) homelike environment and d) pushing against the barriers to CCC. Nadiya identifies the skill of listening along with the attitudes of open-mindedness and willingness to learn as attributes of a CCN. The theme of cultural wisdom is narrated by Nadiya with respect to historical influences, the boundaries of cultural knowledge and importance of communication. In describing the homelike cultural environment of the mono-culture LTCH, Nadiya refers to the physical appearance of the facility, availability of ethnic foods and social activities as creating a setting where the nurse is able to care for residents who share her cultural background. The Ukrainian environment, as Nadiya explains, also helps non-Ukrainian 
staff interact with the Ukrainian residents in a meaningful way. Finally, Nadiya identifies lack of time as a barrier to CCC. The four major elements associated with the delivery of CCC, along with their supporting points, are described below. Following this, Nadiya's overall conceptualization of CCC is introduced and explained.

\section{Attributes of a CCN}

Nadiya's narrative is peppered with references to attributes of a $\mathrm{CCN}$ who routinely includes elements of the residents' culture in the care she provides. Nadiya believes that including elements of culture in care is of great importance for older immigrant adults who reside in LTCHs, as their stay at the facility is not transient, as it would be at an acute care hospital, but more extensive. For the majority of residents, the LTCH becomes their final place of residence. Given this understanding, Nadiya is convinced that residents of LTCHs deserve to receive care that reflects their cultural preferences, as this creates a feeling of emotional tranquility and satisfaction. Nadiya emphasizes this notion when she states, "Our elderly, they deserve and they expect that culturally competent care...it's easier to work with them and provide to them that care, because they are there for a long time and you learn more about them" (Lines 153-155, Interview 1). She places the responsibility of providing $\mathrm{CCC}$ on the nurse, emphasizing that certain skills and attitudes are important for effective provision of CCC. Three main attributes of the $\mathrm{CCN}$ emerge from Nadiya's narratives, the skill of listening, as well as the attitudes of open-mindedness and willingness to learn.

Nadiya identifies that the skill of listening, and an open-minded attitude go handin-hand as the defining characteristics of a CCN. Both must be present for the nurse to practice at the level of a CCN. Open-mindedness is perceived by Nadiya as being both 
vigilant in understanding the sub-text of what is being said, as well as accepting the ideas that may differ from the nurse's own way of thinking. This is particularly important when caring for persons of a culturally diverse background, as they have values and traditions that are unique to their culture. Nadiya explains, "As long as you listen to people, try your best and...let them keep their dignity...that's how you...provide them with...culturally competent care" (Lines 148-150, Interview 1). From her own practice, Nadiya explains, "if I listen and hear what they [the residents] say, then I adjust my care to those beliefs and I include that in my care" (Lines 500-502, Interview 1). By being open-minded and accepting how others think and behave, the nurse becomes familiar with the unique culture of those individuals and is able to incorporate it into her care. It is the skill of listening that aids the nurse in hearing, understanding, and accepting of, then acting upon the needs of the resident are.

Another belief Participant One associates closely with $\mathrm{CCC}$ is the willingness to learn, both from the interactions with the residents and from one's own mistakes. Nadiya emphasizes the importance of learning when looking to provide CCC to individuals whose cultural background differs from your own. Such willingness to learn is of high relevance in Canada, a diverse and multicultural country. Nadiya believes that one can "learn different values and traditions", and use the gained knowledge to provide the care that is expected by the residents. A degree of learning must also be done when interacting with individuals who share one's own cultural background. For example, due the existence of varying dialects in Ukraine, nurses at a Ukrainian-specific LTCH learn the different words and phrases that are familiar to the residents. The $\mathrm{CCN}$ will then attempt to include the specific wording in subsequent interactions with the residents. Nadiya 
explains how willingness to learn is important when caring for clients of one's own cultural background, stating, "I speak Ukrainian fluently, so sometimes when the residents use some words they know from home, like I said...like we said 'old language', those words we don't use anymore, and nobody says them anymore, so I, from the context, I actually use what they means, so I try to remember and again I tell them those words next time I, so it's not a big problem for me. I try to learn, I try to remember and understand." (Lines 280-284, Interview 1). Nadiya demonstrates that she is willing to learn, is open to new ideas and is able to use the skill of listening when providing CCC.

Nadiya's narrative includes a comprehensive explanation of the interconnectivity between the CCN's skill of listening and attitudes of open-mindedness and willingness to learn functioning in a culturally diverse healthcare environment. Nadiya explains:

Canada is such a diverse country, and we have so many different nations living side by side, so....if you trying... if you working in a healthcare, you try to do your best to provide culturally component... [corrects herself] competent care. So that's, that's when you have to learn different values and traditions, and what they do traditionally. You have to be quiet open-minded and accept whatever people like, and how they behave, what they believe, and...you...that's no matter what you think about that, you have to provide the care what they expect. The attitude is very important and being okay with other think, or do, or believe in. And you have to....we have to remember not everybody is the same as we are. But we still treat everybody well. (Lines 101-110, Interview 1)

It is not enough for a $\mathrm{CCN}$ to possess only one of the above-described attributes, explains Nadiya. Excelling in one area while lacking in another compromises the ability to provide CCC. Nadiya notes, "there is people who want to learn, but they might be too closed-minded, or they don't have experience and they don't hear what others say, they just don't want to hear" (Lines 186-188, Interview 1). This explanation alludes to Nadiya's conceptualization of CCC, and emphasizes that bringing together the various skills and attitudes into the caring relationship allows the nurse to provide CCC. 
Developing skills takes time, explains Nadiya. It is through "experience and exposure" that a perceptive nurse learns of the residents' preferences and needs (Nadiya, Line 164, Interview 1). Through listening to the residents, and by incorporating their stories into her actions, the nurse is able to personalize the care she provides and is, therefore, able to offer CCC.

\section{The Nurse's Cultural Wisdom: "I know my people"}

While Nadiya speaks confidently of the skills and attitudes a CCN needs to have in order to provide $\mathrm{CCC}$ to any client, she consistently refers to her own connection to the cultural group as a leading factor in her ability to provide $\mathrm{CCC}$ to the residents at the Ukrainian-specific home where she works. The residents often become akin to family for the nurse. Nadiya cares for the residents "as good as I would care about my parents" (Line 98, Interview 1). This statement is symbolic of the deep level of connection Nadiya has with the residents, where she meet their needs as effortlessly as she would for her parents. Nadiya also recognizes that her colleagues possess an understanding of Ukrainian culture that supersedes acquired skills and attitudes of a CCN, as she explains, "many of us, we listen and we hear, but we also just know" what the residents expect as part of their CCC (Lines 732-733, Interview 3).

What is it that makes it so easy for Nadiya to care for strangers as effortlessly as she would for her parents? It is because, as Nadiya explains, she is Ukrainian herself, therefore she shares the cultural heritage of her clients. Nadiya explains, "I know ours tradition, I know how things were done back home and I know what we believe in" (Nadiya, Lines 98-100, Interview 1). In her day-to-day care, Nadiya relies on her personal identification and experience with the Ukrainian culture to guide her actions and 
decisions. Nadiya comments, "I really think I understand them [residents] so well because I understand their background. I know where they [are] coming from. And it's more than just speaking the same language" (Lines 429-431, Interview 1). As such, Nadiya has made a commitment that she is a guardian for all things Ukrainian. She sees herself as the person who will enact a "way of being" that would be recognizable to the resident as Ukrainian.

Historical influences: The past must be explored. Nadiya recognizes that CCC in the Ukrainian LTCH context means hearing and respecting what has happened before. As a CCN of the same cultural background as the residents, Nadiya accepts that people have diverging experiences with the culture due to historical or social circumstances. This is pronounced in her care of Ukrainian elderly residents belonging to the first wave of immigrants from Ukraine. For example, Nadiya narrates, “ours Ukrainian elderly people, they been through so much, and maybe I didn't live through some of things myself, but I can still understand them and I can understand why they did things in a specific way and a way that only they like to do it. It's just a shared understanding, I would say. It's difficult to explain, really. I don't know how to explain it well. I guess it just in my heart." (Lines 486-490, Interview 1). Despite the inter-generational differences between her and the residents, Nadiya is still able to understand the mind-set of these individuals who share her cultural roots.

Having a personal connection to the cultural group, as well as an in-depth understanding of the intricacies of the culture, is also helpful to nurses in instances where the residents' exhibit responsive behaviors. For example, the participant describes occasions where she brings homemade Ukrainian food for the residents, although she 
anticipates some of the criticism she might encounter about her cooking, since the residents "care very genuinely about our traditions" (Nadiya, Lines 432-433, Interview 1). As Nadiya explains, some residents suggest how to adjust the recipe to make it more authentically Ukrainian. "If I do it wrong, they give me a big lecture...they tell me what to do better", Nadiya explains with ease, holding no contempt towards the residents' constructive criticism of her cooking (lines 368-369, Interview 3 ). She is not dismayed by such comments, as she understands that it is part of the culture of the older adults to guide the young. Nadiya explains that "definitely residents like to talk about food...they will teach you how to do stuff" (Lines 350-351, Interview 3). She does not take their suggestions for how to improve her food as a criticism, rather, she sees this as an opportunity for the resident to "teach her", and therefore fulfill their role as a respected elder. In recognizing the existence of inter-generational differences between the four waves of Ukrainian immigrants in Canada, the participant identifies a "shared understanding" of culture as the guiding the CCC that she offers to the residents. In this instance, Nadiya understands that her role as a $\mathrm{CCN}$ includes bringing the past forward into the present; recipes from the past should be compared and contrasted with those of the present, just as the experiences of the past have shared today's behaviors. The present has no meaning without comparing it to the past experiences of the residents in her care. The past becomes essential, and must be named and examined by the residents.

Boundaries of cultural knowledge. Although the participant verbalizes confidence in her familiarity with the Ukrainian culture, she does admit it is impossible to know everything about a culture. Nadiya says as much when she states, "I'm Ukrainian...it doesn't mean I know everything about my culture" (Lines 166-167, 
Interview 1). Culture is a multidimensional concept, and as such, it places restrictions on how much one may really know about it, whether from personal connection or through objective learning. Geographical boundaries, interpersonal interactions and historical events may all shape and diversify a nations' culture. Although Nadiya has narrated instances of nursing actions that have been shaped by her cultural affiliation, she recognizes that she may not be fully versed in the Ukrainian culture. One such example of a gap in understanding one's own culture is instances when Nadiya encounters residents using Ukrainian words that are unique to their geographical region in Ukraine; the meaning of the words may not be known to the nurse. If such gaps in knowledge and understanding are ignored, this creates potential for miscommunication and adverse client outcomes. However, the nurse's personal characteristics, such as positive attitude, ability to recognize one's own shortcoming and willingness to learn, may help narrow these gaps. Nadiya explains from her own experiences that taking the time to learn about the resident by being attentive in their interactions helps close the gaps. She says, "you just take your time, you sit with the resident when you have a minute, and they going to tell you their story, and from their story you learn about what they, what they expect." (Lines 177-179, Interview 1). While Nadiya may not know 'everything' about her culture, she recognizes that she does have an overall general understanding of what it is to be Ukrainian upon which her care is based. Nadiya demonstrates that CCC is still possible despite lack of knowledge of each and every detail. As a CCN and a Ukrainian, she recognizes instances where there are discrepancies between her knowledge and presenting facts. She then relies on her skills, such as the ability to listen, and her attitude, 
including open-mindedness, to guide her care. By placing the resident's needs at the center of her care, Nadiya continually strives to provide CCC.

Communication: I speak your language. One of the defining characteristics of a Ukrainian individual is their ability to speak Ukrainian. Fluency in the residents' language helps promote the ability of the nurse to provide $\mathrm{CCC}$, making it easier to form connections with the residents. Nadiya ascribes great value to language and the ability to communicate with the resident as part of CCC. She explains her thinking stating, "Language, it's means of communication...so language is important everywhere...in any care...you won't do assessment, you won't know what person wants....or needs, that's why even deaf people...they learn to communicate by sign language...everybody needs to know a language to be able to connect with others" (Nadiya, Lines 663-666, Interview 3). The residents use language to voice their present needs and concerns, as well as their past histories, which may be laden with clues as to the kind of CCC the residents would like to receive. Understanding the needs of the residents as they are being expressed in a shared language makes it "easier to care", explains Nadiya (Line 241, Interview 1), and removes uncertainty out of the interaction. Nadiya draws contrast with the communication boards used in hospital settings for identifying the degree of pain experienced by the person versus the ease of interacting verbally with the resident experiencing pain using their native language. Nadiya reasons as follows, "they have those visual things...chart...but these older people, they don't know how to rate their pain from one to ten....or use that smiley face chart...it's too different for them. But with somebody who speaks Ukrainian, they just say what they want. They have no need to waste their energy translating...thinking how to say something in English." (Lines 622- 
624, Interview 3). The residents are able to "tell you exactly what they feel" when speaking about an experience in their language (Line 271, Interview 1). In this way, the resident's emotional status is expressed and understood.

It is mutually beneficial for both the residents and the staff to share a language. Nadiya comments, "they happy they can understand us, and we can understand them" (Lines 245-246, Interview 1). The participant conveys that, when she is speaking Ukrainian with the residents, she is never concerned that she might not understand what is being said by the resident or that the resident will not understand her. The benefit of sharing a language is also applicable with residents who, due to illness or age, have lost knowledge of English language. The residents expect to be cared for by Ukrainianspeaking staff. This is emphasized in Nadiya's words, "I can see how surprised they are when somebody who doesn't speak their language... residents prefer those of us who fluent in Ukrainian" (Lines 622-624, Interview 3). Nadiya describes the frustration experienced by staff who are not of Ukrainian background when interacting with residents who do not speak English. This frustration is mirrored by the residents, and often results in instances of miscommunication. According to Nadiya, "they get frustrated a lot when they don't understand each other. The staff and residents - when it's miscommunication, that's when problem arise." (Lines 274-275, Interview 1). The ability to communicate is an important element of Ukrainian culture, as Ukrainians are "very vocal people", emphasized Nadiya (Line 239, Interview 1). Sharing a language helps form a connection with the residents and creates an interpersonal platform where CCC can occur. 


\section{Homelike Environment: "A Very Ukrainian Environment"}

Nadiya describes in great detail the features of the LTCH where she works, emphasizing how the Ukrainian environment is promoted at the facility. Among the traditional Ukrainian items that can be found at the home are religious artifacts, including icons and crosses, decorated Easter eggs or pysanku, embroidery and books. As Nadiya explains, “Everywhere you go, it's everything Ukrainian” (Lines 290-291, Interview 1). The presence of the Ukrainian flag is mentioned by the participant with resonance of national pride in her voice, as she says "You see Ukrainian flag outside, it's a must!...it's our pride to have flag outside" (Nadiya, Lines 314-316, Interview 1). When describing how one would recognize that they have come upon a Ukrainian LTCH, Nadiya engages the sense, explaining, "it just smells like Ukrainian home” (Line 293, Interview 1). Adding to the Ukrainian feel of the home is the varied diet of Ukrainian traditional dishes, including borsch, a soup of red beets, holubczi, or cabbage rolls, and varenyky, or perogies. The availability of Ukrainian food at the LTCH, explains Nadiya, is one of the elements considered by family members when selecting a nursing home for their elderly loved ones. Nadiya describes this as follows, "when the families looking for nursing home...for their parents... when they first come, they ask about diet...it's important what will they be able to eat... will certain dishes be served to their parents? We encourage families to come and sit at table when meals served if they visit at a mealtime...they help feed residents." (Lines 351 - 355, Interview 3). Ethnic food is an important element of Ukrainian culture for the residents, as Nadiya emphasizes that it is a common topic of discussion among the residents and the staff. Nadiya says, "Guarantee... at least one resident will talk to me about food during my shift...for sure... maybe even more!” (Lines 356-357, Interview 3). 
The importance of ethnic food as an element of CCC is reinforced in the photos that Nadiya took. The first photograph presented in Figure 2, titled The Ukrainian Lunch shows two staple dishes of Ukrainian culture, including varenyky and borsch, with a with the rims of their plates touching, which is highly symbolic of the connection of these foods to the Ukrainian culture. Often the two are served together, and are frequently offered to the residents of the Ukrainian LTCHs. The second photograph is a composition of Ukrainian embroidery and traditional cabbage rolls. It appears in Figure 3, titled Holubczi ta Vushuvanka, or Cabbage Rolls and Embroidery. Since the photographs had been taken with a disposable camera, the sharpness of the images is somewhat diluted. However, as was explained by the lab processing the photographs, this is likely due to the fact that the photographer was trying to take the shot from up-close. This intention of Nadiya's to focus solely on the food in the photographs further reinforces how essential these dishes are to the Ukrainian culture. Furthermore, Nadiya explains that she had prepared the dishes that she photographed to share with her family. She says, "I made cabbage rolls for everybody ... and then...then we have borsch in the fridge ....and I boil some varenyky...I have them in the freezer...I like to make many of them when I'm alone at home" (Nadiya, Lines 106-109, Interview 2). Having the participant prepare food to be photographed is reminiscent of her tendency to bring food to share with the residents. 


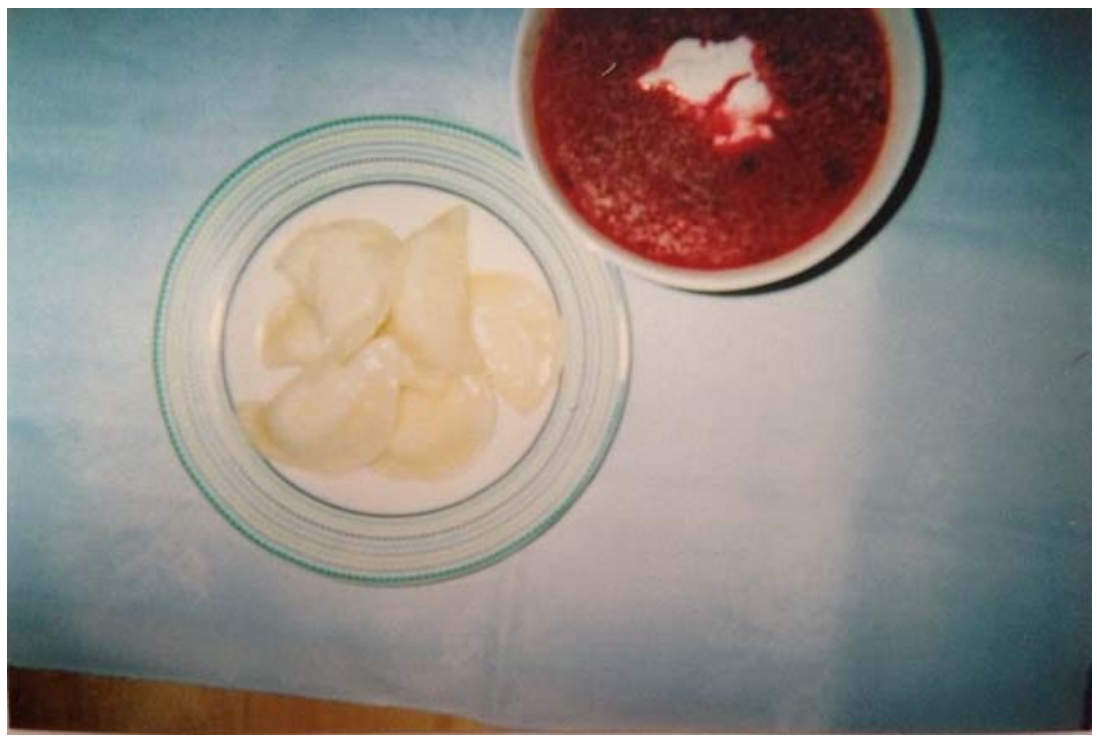

Figure 2: The Ukrainian Lunch

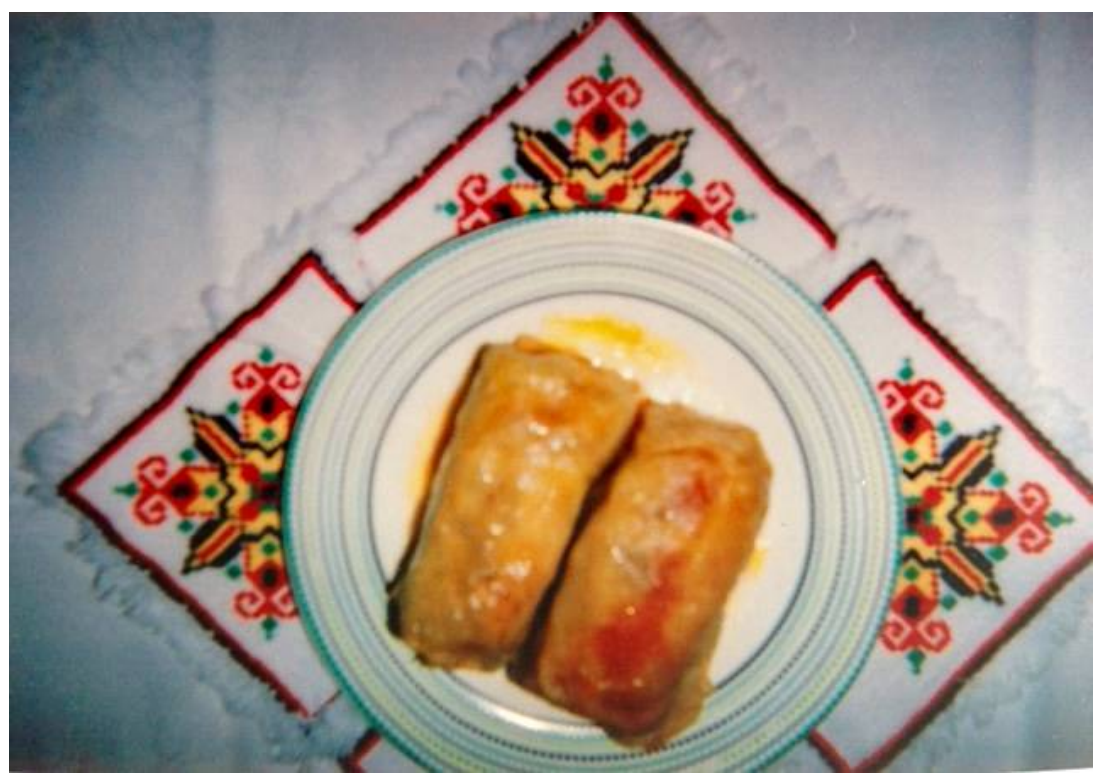

Figure 3: Holubczi ta Vushuvanka

Nadiya explains that, in preparation for the photography activity, she had looked through her old family photo albums and had noted that majority of the images were taken around the table. She explains, "All photographs we have around the table...some of them very funny...just empty plates and people sitting there talking" (Lines 103-104, Interview 2). Sharing a meal, therefore, is symbolic to the Ukrainian traditions and lifestyle. The experiences from Nadiya's personal life associate ethnic foods with culture 
and celebrations. She has seen this in her nursing practice at the Ukrainian LTCHs as well, where she notes, "Kitchen, they try to, as often as they can, they make Ukrainian food. Borsch, that's a red beet soup, varenyky, which comes with different filling, we call holybczi, which is cabbage roll, meat. During holidays, we also...they make traditional dishes. Residents, they LOVE this.” (Nadiya, Lines 295-298, Interview 1). Nadiya emphasizes how important Ukrainian food is to the residents. It is something that brings them happiness, makes them feel "at home".

In addition to physical items and foods, the participant also spoke of social activities that help keep the resident in touch with their cultural background. The widespread availability of cultural elements is seen by the participant as a means for facilitating her capacity to provide CCC. Nadiya explains that the Ukrainian environment at the home reassures the residents and helps the nurse to incorporate elements of culture into her care more readily. Nadiya states, "It's a very Ukrainian environment...so that makes it easy to provide culturally competent care, you have everything Ukrainian there" (Lines 687-688, Interview 3).

The concept of the LTCHs as being a "home away from home" for the residents has been strongly reinforced by Nadiya. The staff encourages families to bring items from the residents' homes to help personalize their living space. Nadiya says proudly, “Every resident's room - it's like a resemblance of their own home" (Line 304, Interview 1). Among the items that the residents bring along are traditional cultural artifacts as well, such as embroidered table runners. Although there exists a variety of styles of embroidery associated with the different geographical locations of Ukraine, embroidery itself is a 
defining feature of the Ukrainian nation. Including familiar, culturally-significant items in the resident's life at the home creates a sense of home and comfort for the residents.

Working in a 'Ukrainian environment' helps nurses who share the cultural background with the residents provide CCC. While the environment is familiar to the residents, it is also familiar to the nurses, as Nadiya notes, "it's probably easier to work with Ukrainian people...it's familiar environment" (Lines 752-753, Interview 3). It is the widespread availability of traditional, cultural artifacts that contribute to the overall Ukrainian 'feel' of the home and make it easier for the nurses to include CCC in their daily routine. Nadiya says that the Ukrainian items surrounding the residents are "dear to their heart", and concludes that "all those things make my job easier...half of it is done already...the residents feel comfortable" (Lines 434-436, Interview 3). The presence of traditional cultural elements within the healthcare environment aids the nurse in incorporating her personal knowledge of the culture into the care she provides and to express her own cultural understanding in the care she offers.

The Ukrainian environment created by the presence of traditional cultural elements also helps non-Ukrainian speaking nurses provide $\mathrm{CCC}$ to the residents. The environment is supportive for non-Ukrainian staff who have developed the attitude of being open-minded and the willingness to learn, two trademark attitudes Nadiya associated with a CCN. Despite being unable to communicate in Ukrainian, the Englishspeaking staff can adapt their care to fit the cultural needs of the residents by including the traditional elements available around them. For instance, they can rely on Ukrainianspeaking nurses to assist with translation, which, Nadiya concludes, improves the 
residents' satisfaction with their care. Nadiya gives insight into the experience of non-

Ukrainians staff in the following exchange:

Maryana: How do you think they manage...like those English-speaking nurses, in such a Ukrainian environment? Like last time, you were telling me that it even smells like a Ukrainian home...but that's probably strange for the Canadian nurses to be in a place that's so oriented to one culture?

Nadiya: You know...for them to provide care...okay, maybe they don't know the language very well...but they are, like you said, in Ukrainian environment...so some of them who has big hearts and want to help people, they ask us if they have question or if they need help. Some leave because it's hard ...but you know, they pay attention and they know routine, and then there are so many things around that are Ukrainian...they just adapt to that environment too. Oh, and then residents get used to them.....at the end, everybody likes each other... so sometimes they understand each other even if they don't speak same language. But, if it would be less Ukrainian-speaking caregivers at our place, it would be worse, I think.

(Lines 645-658, Interview 3)

The Ukrainian environment of the home therefore benefits both the residents and the staff, as it removes the burden of finding ways to introduce cultural features into the care. This reduces the risk for misunderstanding of cultural preferences. Additionally, a sense of mutual respect develops between staff and residents in the safe and familiar environment within the home.

\section{Pushing Against the Barriers to CCC}

Nadiya has explicitly identified lack of time as a barrier to providing CCC. She explains, "there is never enough time to do everything for the residents in your eight hour shift" (Lines 337-338, Interview 1). When acting out the regulated nursing activities, such as medication administration, Nadiya emphasizes their importance by saying "you cannot be interrupted", yet she feels pressure from family members to devote more of her time to the residents' personal and social needs. Although there are general nursing activities, which nurses aim to fulfill throughout their shift - such as medication 
administration - lack of time restricts the opportunity to inject moments of one-on-one, personalized care into the routine. Such personalized interactions, although not solely healthcare-related, are meaningful to both the residents and the caregivers, as they help build relationships. Nadiya explains that CCNs look for the opportunities to inject moments of tailored care, and would act upon them, however, they may not be able to do so due to lack of time. An example of personalized care could be reading to a resident in their native language. Nadiya notes, "if I have the time, I will ask them about it... and we can talk about what they read...or...or what I read somewhere...you know, it helps make connection with them" (Lines 283-285, Interview 3). When probed to discuss how often she reads to the residents, Nadiya explained that this happens "only sometimes". She does indicate, however, that she would welcome the opportunity to have more meaningful moments with the residents.

Lack of time, as a barrier to providing CCC, is further complicated by the staff shortages that predominate the healthcare environment. Nadiya repeats the message "We don't have enough staff' several times throughout our conversations. Although she does not refer to any instances where the residents' care has been compromised by the lack of staffing, it becomes clear that having a greater staff to resident ratio, in the participant's opinion, would benefit both parties. Nadiya clearly conveys this message when she says, "In my opinion, we need more nurses, more PSW [personal support workers]" (Line 356, Interview 1). Having more nurses and personal support workers, according to Nadiya, would release more "time for those extra thing $[\mathrm{s}]$ which is important in resident" and that contribute to their healing, such as having meaningful conversations with the residents (Line 357, Interview 1). Otherwise, Nadiya mentions, nurses feel obligated to set time 
restrictions on their interactions with the residents in order to be able to fulfill the activities outlined in their job description. For example, some residents "just want to talk", but Nadiya warns "you really have to set time limit, because otherwise...nothing will get done" (Lines 353-354, Interview 1).

Additionally, having time available for the residents' families would help alleviate some of the stresses and anxieties they have as the result of placing elderly family members in LTCHs. Nadiya explains that it is not part of Ukrainian culture to place elderly parents in a nursing home, yet, when faced with the realities of life, many children in Canada choose this alternative. She elaborates, "it's really not part of our culture to put older parents at home. So...that's just Canadian reality, I guess. Like we cannot afford to keep elderly at home, and somebody stay with them" (Nadiya, Lines 363-365, Interview 1). As a result, the family members have "big guilt", as Nadiya labels it (Line 365, Interview 1). The family members then attempt to purge themselves of this guilt by engaging the nurses in extensive conversations regarding the parents' well-being, or by expecting that the staff at the home will dedicate more time specifically to their loved ones. Nadiya analyzes the situation, explaining "they try to compensate their absences with somebody's presence” (Lines 369-370, Interview 1), meaning the families, who miss out on time spent with their elderly loved ones, expect the nurses to spend extra time with them. In many instances, it is not feasible for staff to fulfill such expectations due to lack of time. This concerns Nadiya, who says "we do to the best of ours ability to compensate for the loss of family, communication. But it's not...not always happening" (Lines 369-371, Interview 1). It take time to become familiar with the residents and to form therapeutic relationships, however, as nurses face lack of time as a barrier in their 
care, they miss out on opportunities to provide the residents with instance of CCC. The residents themselves, along with their families and the HCPs are affected by the lack of time, which makes it a challenge to provide CCC.

\section{Nadiya's Conceptualization of CCC}

The themes described in the preceding pages have emerged through Nadiya's narrative as elements that either facilitate or hinder a CCN's ability to provide CCC. Nadiya provides explanations for the list of skills and attitudes she associates with a $\mathrm{CCN}$, along with a description how the various elements of culture, including language and historical context, can shape a nurse's understanding of the client's cultural background. She acknowledges the potential for diverging cultural views to exist between clients and care providers who share a cultural background. She also describes the details of a LTCH that create a homelike feel for the elderly Ukrainian residents. Finally, Nadiya introduces lack of time, and the factors that compound it, such as staffing shortage, as a barrier to CCC. Through all the descriptions that she provides, Nadiya weaves an explanation of how she defines CCC. In the following section, Nadiya's conceptualization of $\mathrm{CCC}$ as the act of keeping the golden middle is introduced and explained.

\section{Zolota Seredunka: CCC as Keeping the Golden Middle}

In her nursing practice, Nadiya makes no conscious distinction between care that is culturally competent versus that which is routine. Rather, she directs her efforts at providing care that is void of extreme afflictions, and is instead a fine balance of human empathy, cultural understanding, clinical proficiency, and personal respect. In her encounters with the residents of the home, Nadiya strives to maintain what she calls 
Zolota Seredunka, or the Golden Middle. "Zolota seredunka," explains Nadiya, "it's all about balance.... and harmony. And that's what we try to do with our residents... we try to give them a home... but also we provide them with healthcare needs" (Nadiya, Lines 598601, Interview 3). Attending to the residents' healthcare needs, in this case, means using nursing skills, such as assessment, to promote the physical well-being of the individual. Ensuring that the residents feel 'at home' at the LTC facility requires that the nurses introduce into the residents' day-to-say life moments of care that reflect the individuals' cultural preferences. Maintaining the golden middle, is, therefore the essence of CCC.

In her narrative, Nadiya positions the residents - care recipients - as the focus of her care, explaining that CCC is about fulfilling the needs and culturally-bound preferences of the residents. The residents are entitled to be treated in a manner that is respectful of their preferences. It is the resident's choice that directs the kind of CCC the nurse is striving to provide. Nadiya equates CCC to holistic care in that it expands beyond the boundaries of traditional medical care and routine nursing skills, which care for the body, to include acknowledgement of mind and spirit. "Nursing care...it doesn't stop at medication or bed bath...you have to care for...oh! You have to do holistic care! You care about body, mind....and spirit" (Nadiya, Lines 394-395, Interview 3). This is achieved by incorporating elements, such as foods and traditional customs that are familiar and valuable to the resident into their day-to-day lives in a LTCHs. "I think that food and habits....they are very important and we try to include them in the care that we offer", elaborates Nadiya (Lines 392-394, Interview 3). Elements that are valuable to individuals are normally ever-present in one's life, and, thus, shape the individual's behaviors and habits. Nadiya believes that preserving the habits that have come to define 
a person post-transition into LTC promotes that individual's healing. For example, some residents have a common habit of reading in their leisurely time; Nadiya promotes this pattern of behavior, as she notes, "people enjoy reading....and it's part of their healing, kind of like meditation.... or yoga for brain. This is something they like to do. And at our home, we try to promote this home-like environment...and all things...the activities they did at home" (Lines 262-267, Interview 3).

Keeping the golden middle also means letting the client's expectations lead the direction of care without jeopardizing the nurse's own needs and identity. The expectation that culturally-tailored care, as well as traditional cultural elements, will be widely accessible is one of the factors considered in the decision-making process when an older immigrant adult transitions into LTC. The responsibility to enact CCC, to a significant extent, falls on the frontline staff, namely nurses. It is the nurses' mandate to fulfill those expectations and to provide care that the residents are seeking. Their ability to do so is highly relevant in LTC settings, since the resident's stay within the healthcare setting is extensive. Nurses take on the role of liaisons, striving to strike a balance between the resident's expectations, professional care standards, organizational mandates and personal cultural affiliations.

As part of her ability to keep the golden middle while providing care to residents at an culturally-specific $\mathrm{LTCH}$, the nurse relies on her own intimate grasp of the culture as one of the sources of information. Nadiya readily identifies significant elements of Ukrainian culture; for example, she explains, "to Ukrainians, things that represent their identity is food, and observing traditions...it's reading classical poems...embroidery" (Lines 404-405, Interview 3). Being of Ukrainian origin herself, Nadiya is able to include 
elements of Ukrainian culture into her care in a fluent manner. Nadiya explains, "I take my knowledge of my culture with me when I look after the residents" (Lines 225-226, Interview 1). Furthermore, having such deep and personal understanding of the intricacies of the culture means that many of the elements that she includes fall outside of the mainstream understanding of Ukrainian culture. There are aspects of culture that are so unique that they cannot be taught or explained to those who do not belong to the cultural group. It is only through prolonged immersion in the group that an individual learns the underlying subtext of certain cultural elements. Sharing the cultural background fosters the development of a mutual understanding, which facilitates CCC. "When we understand each other, that's when it becomes easier to care for them [the residents]", says Nadiya (Lines 240-241, Interview 1). An element of keeping the golden middle therefore, is investing enough of her own thoughts, efforts and knowledge into the care that she providing, without trespassing the boundaries of therapeutic relationship, while also maintaining the integrity of her own persona. As a Ukrainian herself, Nadiya is able to understand the subtext and the significance of individuals' behaviors and actions. There is often hidden meaning in how cultural traditions are expressed. She demonstrates a deep perception of what it means to be Ukrainian, and is able to interpret the residents' needs based on her own cultural knowledge and personal values/beliefs of what it means to be "Ukrainian". When a nurse is receptive to the needs of the resident, she is able to shift her care from moment-to-moment, so that, overall, she provides the care that the resident wants and is respectful of the residents' cultural background. 
Nadiya has been able to capture her perception of zolota seredunka in a single photograph, the image of which is included below (Figure 4).

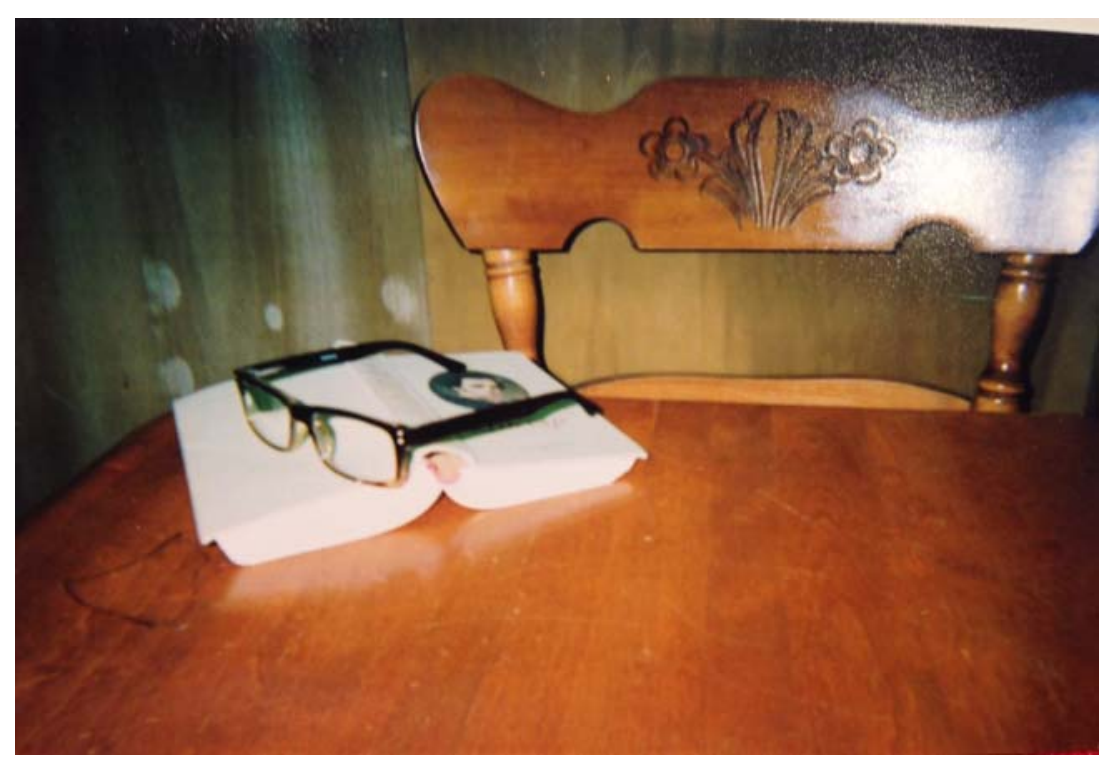

Figure 4: The Kobzar and the Resident

Upon first glance, it appears that the photograph is just a collection of household furniture and material items. There is, however, cohesion in the photograph - the chair is tucked in close to the table, the book, a Kobzar, is off to the side, the glasses rest carefully atop the book. This photograph is meant to represent a scene Nadiya has seen frequently in her practice at the Ukrainian LTC home. She says as much, explaining "it's resemblance of behavior I've seen" (Nadiya, Line 253, Interview 3). As Nadiya and I examine the photograph, she urges me to engage my creative power, to imagine that which she has left out of the photograph - the person in the chair. The seat has been left empty intentionally to allow the audience to envision the older adult that might occupy the chair. Nadiya thinks of her residents, while I see the face of my grandfather. In Nadiya's explanation, she imagines an older man is sitting in the chair and he appears relaxed and comfortable in the environment he is in, as he has the opportunity to engage 
in an activity - reading - that he enjoys. His autonomy is preserved as he is reading a Ukrainian book of iconic historical significance in the comfort of his 'home'. The residents' freedom to carry on with routines and habits is "part of their healing", explains Nadiya (Nadiya, Line 262, Interview 3). When asked to describe how she, as a nurse, fits into the image that she captured Nadiya explains that her role is to allow the older adults to continue to live their life as they had done prior to relocating to the LTC home. "It's part of my nursing care", she elaborates, "to let the man I imagine to be in this chair to do things the way he did them all his life" (Interview 3). In recognizing that the LTC institution is a home for the residents, she gives them the power to be in charge of their own lives. Examining the photograph, I note that it is taken from a close-up range with the photographer - the participant - positioning herself directly across from the invisible client in the chair. This viewpoint gives me further insight into how the participant positions herself as a nurse in the care of the residents - they are her equals. There is no power imbalance between the nurse and the resident, rather they sit at the same level. By putting the control of the direction of care into the hands of the resident, Nadiya allows the residents' needs to shape her care.

The image captured by Nadiya was "made" by her, as she arranged the book and the glasses to be representative of scenes she has witnessed at her workplace. Nadiya explains that she had deliberately chosen the book in the photograph, the Kobzar, which is a collection of poems by Taras Shevchenko, a Ukrainian poet and painter. The book itself holds deep meaning among Ukrainians as a symbol of rebirth of Ukraine as a free nation distinct from Russia (Internet Encyclopedia of Ukraine, 2001). Anecdotally, the Kobzar is frequently placed at the level of the Bible in Ukrainian culture; a copy of this 
book can be found in most Ukrainian homes, while a 'true patriot' of Ukraine can recite by heart the central poem of Shevchenko's works, the Zapovit. The Kobzar has been translated into a number of foreign languages (Internet Encyclopedia of Ukraine, 2001). An English version, titled The Poetic Works of Taras Shevchenko: The Kobzar, was translated by Andrusyshen and Kirkconnell and published by University of Toronto Press in 1964. The symbolism inherent to the Kobzar fits well with Nadiya's message of promoting the residents' autonomy. 


\section{Vera - Bipa}

As the result of the first level analysis, several elements supporting Vera's conceptualization of CCC came through in her narrative, namely a) collegial support b) client's needs through personalized lens, c) the Ukrainian setting and d) the description of the CCN as a gardener tending to the different flowers. Similar to the description with Participant One, visual analysis of the photographic data is used to augment the elements identified through categorical-content analysis. The four major elements are introduced first, followed by Vera's overall explanation of what it means to provide CCC to elderly Ukrainian immigrants.

\section{Collegial Support: "We share the care"}

Collegial support and collaboration are essential for nurses to provide CCC. Vera made frequent references to her colleagues through our conversations. The collegial collaboration that Vera describes often transcends the physical support, such as helping to position or move residents, into the realm of interpersonal support. For example, the colleagues share information about the residents' preferences and needs among themselves. Vera emphasized this point when she said, "Teamwork in our environment...is so important...from starting to moving residents...it's muscle work... and you need help... and then some extra information about residents...we share that care" (Lines 319-321, Interview 1). The support provided by colleagues may take on different forms, depending on the presenting situation and the client's needs.

In addition to collaborating for the benefit of the resident, the colleagues are also keenly aware and respectful of the needs of their coworkers. By sharing their own experiences and understanding the staff help each other provide CCC to the residents. 
Vera spoke highly of her colleague's leading role in helping her acclimatize to the work environment, identifying them as sources of significant information about the residents in times when there was lack of formal training offered by the administration. The following excerpt from our conversation emphasizes the notion of colleagues as instigators of onthe-job cultural training for Vera.

Maryana: Then how did you learn how to provide culturally competent care to your patients?

Vera: Our residents? From co-workers. We have very good developed team in our place. There is people who work there for long time, and they know residents. Experience! They have best ways to do stuff...I say this before to you...you learn how the resident like things, what they needs are, and then you tell your coworkers. You share this and that's how you help each other. When I first come, everybody help me...so now I can help new staff who come...

(Lines 371-378, Interview 1)

As a rooted member of the staff team, Vera has now taken on the responsibility for sharing her knowledge and insight with the new staff, she explains. According to Vera, “...I am very lucky. I work in institution where everybody is genuinely happy to work there. Everybody so helpful, and we all learn from each other, we all share our knowledges, and I am very, very lucky I find our place.” (Lines 430-432, Interview 3). The communal delivery of care promotes the CCC offered to the residents.

Vera was also candid about the staff's dedication to the residents, as well as their tendency to go beyond the call of duty for their residents. She describes an instance where, due to staff shortage, a resident became upset when she could not participate in an activity happening downstairs. Vera goes on to say, "Imagine how good our staff is? One of the activities coordinators, she go and sing with this resident in her room" (Lines 440441, Interview 1). When I asked Vera if she has seen her coworkers provide CCC to the 
residents, Vera responded without hesitation, "Basically, all the time, they take every opportunity to be helpful for our residents. They will dance and sing to make them happy. Once somebody [staff] goes back home, they bring candies back [for the residents]!" (Lines 447-449, Interview 3). Vera notes that it is these moments of care that go far beyond the job description that represent CCC.

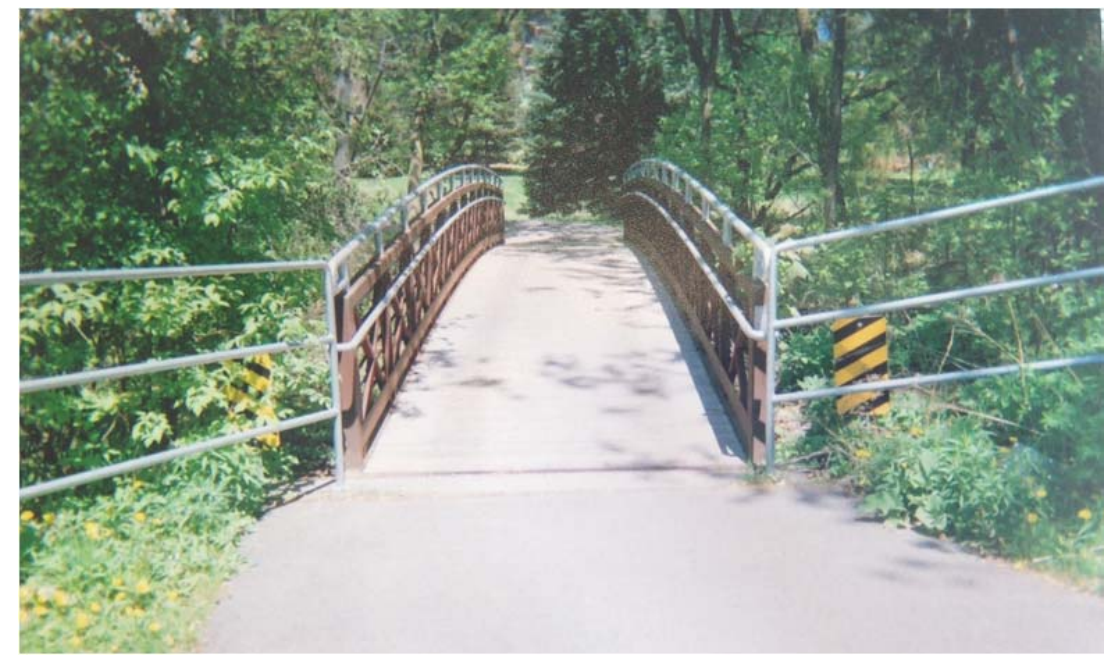

Figure 5: Into Tomorrow

Vera used the image of a bridge as a metaphor for the collegial support in providing CCC. The photographs, "Into Tomorrow" and "We are the Banister", have been captured with a focus on different details of the bridge, and are meant to represent the views of the residents and of the staff, respectively. In Figure 5, the viewer is positioned facing the long stretch of the bridge, leading to the other side. The length of the bridge is symbolic of the journey that the residents undertake during their stay at the LTCH, explains Vera. 


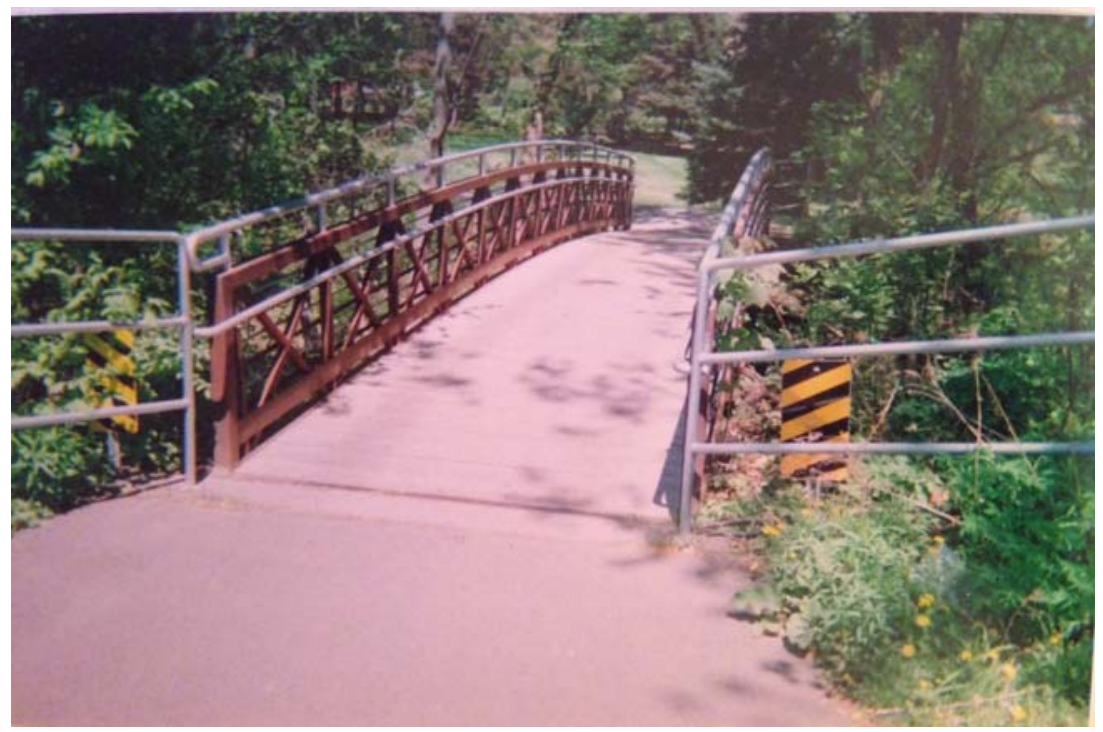

Figure 6: We are the Banister

In Figure 6, the rails of the bridge, or the 'banister' as Vera calls them, are clearly seen in the focus of the photograph. The rails are meant to symbolize the nursing staff of the home who ease the residents' journey by lending support and making them feel safe and comfortable. Vera poetically identified the role her colleagues and she have day-today on the journey that the residents take, as providers of support and protectors of safety.

Vera: ...what I think of this bridge...we all, healthcare workers...we all stand side by side, very tightly to form this banister... and residents, they walk over, crossing that bridge [she moves her finger along with photograph of the bridge]... and they know you there, and they safely go to tomorrow, whatever it will bring to them, because they know somebody will always be there...and sometimes, when you standing there, you can be banged by a wheelchair, or cane...but you stay there....because you make somebody's life better and safer...and they don't know what will be at the end, but they just go there....and at the end of somebody's life .... we nurses stand there to guard their safety.... and if one of them needs help along the way, you push that wheelchair for them to go further.

(Lines 367-376, Interview 3) 
Combining the narrative with the photographs of the bridge creates a powerful image of the extensive interconnection between the staff themselves, as well as between the staff and the residents of the home. Together, the staff delivers CCC.

\section{Client's Needs Through Personalized Lens: Knowing the Expectations}

The nurse's strong personal connection to the cultural group she is providing care for, which forms as a result of the nurse herself sharing the ethno-cultural roots of the residents, elevates the quality of care. Throughout the narrative, Vera refers to the residents as "our people" or "my people", which emphasizes her strong personal connection to the Ukrainian community. According to Vera, CCC means doing "more than just medical care" (Lines 121-122, Interview 1). It involves the ability to anticipate the needs of the residents, which is more likely to happen when the nurse herself navigates through life with a similar set of beliefs, values and expectations as the residents. "You sometimes do something for them because you anticipate what they want...just because you know how things are done", explains Vera (Lines 156-157, Interview 1). In her care of culturally similar individuals, the nurse is able to demonstrate a great level of respect, both "to person as human being...but also respect to the person background" (Lines 144-145, Interview 1). There is also an element of culturallymitigated reciprocity that occurs within the ethno-equivalent nurse-resident relationship, as the nurse learns about the individual, but also about the varied cultural aspects of their shared ethnic group. Vera admits as much when she says, "I learn so much history about my country from these people" (Lines 351-352, Interview 1).

Sharing cultural background with the residents of the home provides the nurse with a unique advantage of being able to interpret the clients' needs through a 
personalized lens. This is particularly relevant when caring for individuals with agerelated mental deterioration or memory-loss. A culturally-matched nurse will provide CCC in most situations because she can anticipate the client's needs. The nurse functions at this higher level of care "just because you know how things are done" (Line 156, Interview 1).

When I asked Vera to give me an example of a situation where she had anticipated the resident's needs, she shared a beautiful story of an elderly woman, Mrs. $\mathrm{H}$, whose condition had deteriorated to a point where she was no longer able to do things for herself. In instances where Mrs. H would become abrasive, Vera and her colleagues brought her warm milk with honey. The sweet concoction soothed the resident, as it is reminiscent of a comfort routine Mrs. $\mathrm{H}$ acquired in her childhood from her mother, whom she had lost during World War II. In her recollection, Vera demonstrates how knowing the resident's past history helps her provide care for that individual in times when the resident is no longer able to voice her own needs. Through this action, the nurses are supporting the resident's dignity and identity. While the custom of drinking milk with honey is not a traditional Ukrainian behavior, the participant has been able to incorporate the resident's own culture into the caring relationship in a meaningful and constructive manner.

When referring to the importance of understanding the resident's needs, Vera mentioned the sharing of a language as an essential component of CCC. Vera believes, "it's a big benefit if you speak the language" (Line 589, Interview 3). While it was not a mandatory requirement for staff to speak Ukrainian, Vera recalls that her fluency in Ukrainian was considered a great advantage by the administration when she was hired by 
the $\mathrm{LTCH}$. Vera explains it is a "big privilege that we can speak to...residents in Ukrainian, and also convenience for them. They enjoy that!" (Lines 604-504, Interview 3). The ability to converse in Ukrainian removes the communication barrier that nurses frequently encounter when caring for older immigrant adults of diverse cultural backgrounds. Vera notes with some certainty, that, when training a nurse on CCC, "You won't be able to teach language" (Lines 420-421, Interview 1). Language is a complex entity, and, as such, Vera believes teaching only certain words or expressions to nurses who are foreign to a culture will not yield the same deep level of mutual understanding that develops when a nurse is fluent in the resident's language. When the nurse and the resident come from the same cultural group, a deep interpersonal connection, rooted in a shared understanding, facilitates the provision of CCC; this is particularly possible since barriers, such as language disparity that commonly predominate in therapeutic relationships when the clients and the HCPs are of different cultural backgrounds, are reduced.

\section{The Ukrainian Setting: My House, My Rules}

The ability of nurses to provide CCC to elderly immigrant residents, according to Vera, is facilitated by the home-like, culturally-rooted environment of the LTC facility. Among the features that help define Vera's workplace as a Ukrainian home is the availability of cultural elements like a chapel and the offer of Ukrainian foods. Vera explains that the home where she works was been built in Ukrainian style and, as such, it was "really built for our Ukrainian people" (Line 209, Interview 1). Incorporated into the structure of the home is natural wood, which, as described by Vera, complements the modern interior of the home. The chapel serves as a place of worship and provides the 
residents, staff and families a chance to participate in the various traditional celebrations, including the Rights of Passage. As Vera explains, some families hold panachuda, or funeral mass, at the chapel, which gives the opportunity for the residents with reduced mobility, as well as for the staff, to say their farewells to the deceased residents. Vera explains, "here, everybody brought down in wheelchairs to say good-bye... and then staff come too..." (Lines 253-254, Interview 1). Over the course of their stay at the home, a relationship grows between the residents and the staff, and the availability of a chapel onsite where a funeral mass may be held gives the staff the chance to mourn the loss.

The home also offers some Ukrainian foods as part of its menu. Food holds great importance for Ukrainian people, as there is a number of dishes traditionally associated with Ukrainian culture. Furthermore, the majority of the holidays that are celebrated by Ukrainian people have specific dishes traditionally associated with them. For example, Vera mentions kutya - a porridge-like dish of wheat berries with a sprinkling of poppy seeds, nuts and/or raisins, sweetened with honey - as being a traditional staple at the Ukrainian Christmas table. This dish is, therefore, served at the LTCH for Christmas dinner as it would be in any Ukrainian home.

Vera, however, warns that the diet at the home does not consist of only Ukrainian dishes. "We have some meals which are Ukrainian...cabbage rolls and varenyky...but not that often," explains Vera, since "our Ukrainian food is very complex to make" (Lines 275-277, Interview 1). She goes on to justify the varied availability of Ukrainianspecific food by noting, "this is older people, they can't have heavy food too much" (Lines 277-278, Interview 1). This statement stresses that the desire to promote Ukrainian culture through availability of Ukrainian food is mitigated by the health needs of the 
residents. For instance, Vera mentions that soup is a regular part of the residents' diet. Still, the dishes that have been named - cabbage rolls and varenyky, or perogies as they are commonly known in the West - are symbolic of Ukrainian culture worldwide.

\section{A Gardener Tending to the Planter: The CCN}

When describing the different elements associated with $\mathrm{CCC}$ - such as the homelike environment of the LTCH institution or the support of colleagues - Vera consistently identifies the $\mathrm{CCN}$ as being integral to the availability of CCC. It is, after all, the nurse who is able to artfully bring together the various aspects of the environment into the single moment of care for the resident. The ability to listen to the resident's needs is strongly emphasized by Vera, who says, "You need to teach nurses to ask questions, and be good listeners... You know, you would teach them importance of customs...so they know that it is important to pay attention to those....and then any nationality they work with...they just remember to pay attention....and don't do things first and ask questions later." (Lines 422-426, Interview 1). By paying attention to the said words, the listener can direct future actions in accordance to the preferences voiced by the speaker. Vera reinforces the importance of good listening skills of a $\mathrm{CCN}$ several more times throughout our conversations. For instance, during our final interview Vera reminds me, "You just have to listen to them [the residents] and they will teach you" (Line 244, Interview 3).

A CCN described by Vera also displays the right balance of respect and empathy towards the residents. Whenever Vera spoke of empathy as a defining feature of nursing care, it was closely tied to the concept of respect for the individual, their personal background and their needs. The word 'empathy' was also among the list of words Vera 
had mentioned during our first interview when I had asked her to define CCC from her own standpoint. According to Vera, empathy is so closely associated with nursing care that it is the standard expectation. She comments, "Empathy...that's the word which very tight to nursing, and that's what expectation for us...to provide that kind of care. (Lines 143-144, Interview 1).

The ability to demonstrate respect and empathy allows the nurse to provide CCC even in instances when she is no longer caring just for residents of her own cultural background. Vera explains, that in a multicultural society, there are people who "have different habits, and they look differently sometimes, and um...they have different color and shapes, and that's what we have to respect" (Lines 190-191, Meeting 2). The ability to be respectful and empathetic is also highly relevant when the individual's health is declining; Vera believes that in such moments, “your care shouldn't be annoying, or too intrusive, but should be empathetic in every way" (Lines 190-191, Interview 2).

To convey her message of respect, empathy and understanding of the residents, especially those of diverse cultural backgrounds, Vera uses the image of an assortment of flowers nestled together in a planter seen below (Figure 7: The Diverse Planter).

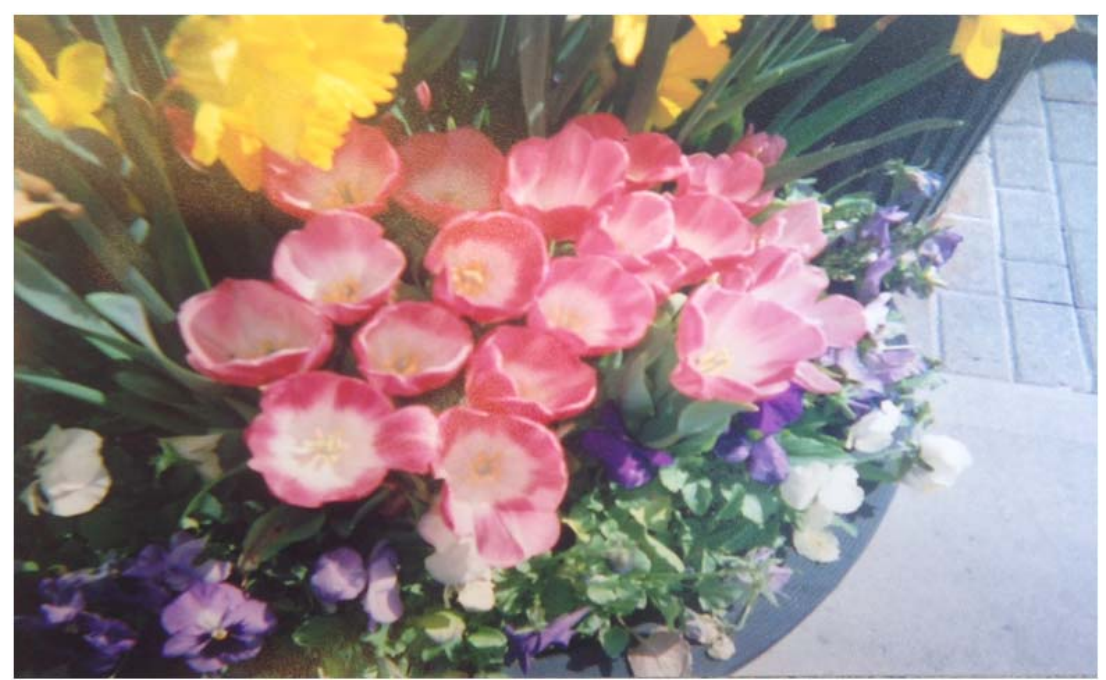

Figure 7: The Diverse Planter 
Vera recognizes that, multicultural society like Canada, there exist LTCHs that bring together residents of different ethnic and cultural backgrounds under one roof. Caring for such a cornucopia of cultures presents a challenge to the nurses, who must adjust their care approach between each individual resident. In this respect, Vera draws similarities between the work of a gardener, tending to the different flowers in a planter, and a nurse in a diverse $\mathrm{LTCH}$, explaining that they both need to have a strong understanding of the needs of each of their individual patrons. Just as the gardener carefully tends the flowers in the planter - all of which are of different varieties - so does a CCN who employs vigilance in her care of the culturally diverse residents. The needs of the residents may change from moment-to-moment, from person-to-person, but the nurse is respectful of and empathetic towards the residents, and is therefore able to provide the care that meets their needs. When reflecting on the message of The Diverse Planter, Vera says:

They put in that planter about five varieties of flowers, and they all live together and grow nice, and they thrive, and why it's like this? Because they given good soil and lots of water. Gardener, he must know what kind of flowers they can put in the same pot so they will be happy together. That's how we should know and try to learn..... what our residents like...and what they want...and what they need to be happy. (Lines 143-147, Interview 3)

In her explanation of the photograph, Vera positions herself, along with other nurses, as the gardeners who work to ensure that the flowers within the planter grow in harmony. Vera believes that, despite her extensive experience of providing CCC to the residents at a Ukrainian-specific $\mathrm{LTCH}$, as a Ukrainian herself she would find it a struggle to care for residents in a non-Ukrainian facility. Vera explains this as follows, "if tomorrow, if I have to go to Chinese nursing home...even with my big experience in Ukrainian nursing home, I don't know how to function there. It's too different...I don't 
know their language" (Lines 388-392, Interview 1). Yet, she maintains that developing the ability to be respectful and empathetic, as well as the skill of being an active listener, would aid her in providing CCC to a diverse group of residents. It would be a matter of time, however, before she could familiarizes herself with the needs of the residents and is able to act upon those preferences.

\section{Vera's Conceptualization of CCC: Supporting the Livinghood}

Vera recognizes that CCC is supported through such attributes such as collegial support, anticipating the client's needs, the cultural environment of the LTCH and the role of $\mathrm{CCN}$. As our conversations progressed, they developed new dimensions, and Vera introduced the concept of livinghood into her narrative. This became the major theme of our discussions, as Vera believes that the aim of CCC is to support the livinghood of elderly adults. The description of the concept of livinghood is given in the following pages.

\section{Livinghood: A New Perspective on CCC}

Livinghood, according to Vera's explanation, refers to the unique needs that are commonly associated with the various stages of life. While people at different stages in their lives have varying needs, those needs are similar among all individuals belonging to a particular stage of life. For instance, while the primary needs of infants' revolved around being "cuddled and changed", this is different from the needs of the elderly adults group. Vera verified that the needs of the older adults are an extension of the activities of daily living taught to nurses: "they have to be warm, they have to have food, they have not be in pain, and they have to be respected" (Lines 265-266, Interview 3). In our conversation, Vera identified what she believes are the core needs of the group of older 
adults. Vera explains "we should know that elderly, they have their own demands, because they are not as strong, they rely on somebody for their support, so that's why we provide for our elderly" (Lines 287-289, Interview 3). Vera believes that the elderly, due to the physiological and psychological changes associated with their advanced age, are in a position where they need the support of those who are more able, such as nurses. It is, therefore, the main objective of CCC for the CCNs is to support the livinghood of older adults. This notion seems to go beyond the principles of body care, or even PCC. Vera appears to use livinghood as a means of providing care that is deeply-felt and spiritual. It seems to blend the boundaries between subjective understanding and feelings with objective knowledge of the needs of the other.

Vera does not differentiate between the needs of older adults and those older adults who are of immigrant background. Vera explains, "we have to remember, all human being is the same, we have to respect our elderly in this country, because they work together to provide what we have right now" (Lines 291-293, Interview 3). She is quick to point out though that respect for the elderly goes deep below the surface of common politeness. She notes, “And respect...it's not just...you let them sit on streetcar because they standing next to you, it goes more...so you do it to make them feel safe and happy" (Lines 295-296, Interview 3). Fulfilling the needs of the older adults, as explained by Vera, means promoting their safety and happiness, which, in turn, supports their livinghood.

Through Vera's explanation, it becomes evident that, in order to understand the needs of a person, one must examine the cultural 'roots' of that individual. Vera believes that, just as the roots of trees stem from the same soil, so do people's roots come from the 
same source. While there may be different branches that span from the roots - meaning the person's life story may vary slightly - the roots remain the same. Therefore, "as long as we find out about those roots, we will know how to treat that particular nation" (Lines 262-263, Interview 3). The primary source of information about the person, as Vera explains, is "First of all, [the] person himself. The human being. You learn so much information from communication with people....You just have to listen" (Lines 281-281, Interview 3). Vera reinforces this point throughout our conversations, noting, "You just have to listen to them and they will teach you" (Line 244, Interview 3). On the topic of livinghood, Vera concludes, "If you know who they are...then you give them what they want" (Lines 267-268, Interview 3). Vera represents her conceptualization of livinghood through a series of photographs of trees, found below. The bright blue background of the photographs creates a feeling of serenity in the viewer.

Vera believes livinghood is a continuation of one's life, and as such, it is a stable feature. The trees themselves are intricate, with the trunks being of different shape and width. The roots of the trees are deeply embedded in the surrounding soil, which hold the trees firmly in their place. There are small leaves beginning to cover the branches - signs of life. The overall appearance of the trees is reflective of the differences that exist between the people, yet all three of the captured trees have similar needs - water, sunlight, wind and soil. Interestingly, all three of the trees are in the foreground of the photograph, nearly centered to the image - this resonates with Vera's belief that the resident is the primary source of information about their preferences. 


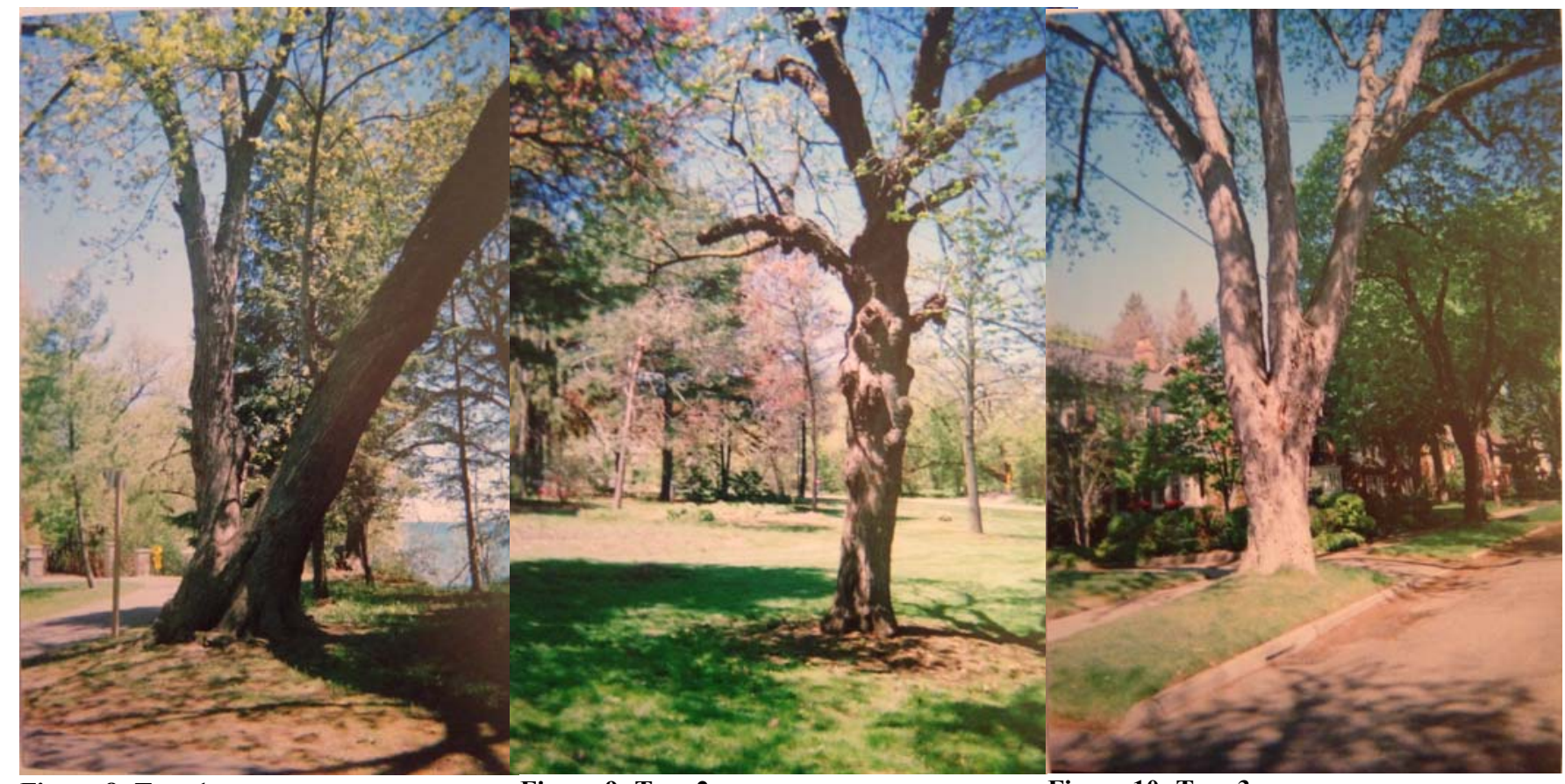

Figure 8: Tree 1

Figure 9: Tree 2

Figure 10: Tree 3

Vera provided a summary of the phenomenon of livinghood, and how it can be

promoted through $\mathrm{CCC}$, when I asked her to share with me the overall message she

wanted to convey through the photographs. Vera said:

Whatever you reach certain age, during your lifespan, people have certain demands... and there is of course difference in cultural traditions, but all the elderly, they need to be protected, they dignity needs to be preserved, they need to have somebody who listens to them...elderly people like to talk, and they have whole life to talk about....all experience to share...depending on their education, and where they were born, and who were their relatives... and all that kind of stuff...and, if you know about roots of that person and in what ground those roots lies, you probably would be more efficient in making them happy.

(Lines 415-422, Interview 3)

In discussing the concept of livinghood, it became apparent that providing CCC to the elderly adults, according to Vera, promotes their livinghood, their very essence, far beyond their name, their heritage, or their preferences.

\section{Nadiya and Vera: Different Homes, Distinct Personalities, Echoed Themes}

The first part of this chapter has described the inter-related elements associated with conceptualization and delivery of CCC as interpreted through independent 
categorical-content analysis of the two narratives. This first level of analysis completed with each of the two sets of data has been supplemented with inferences from visual analysis of the photographic data. During the data analysis process, it became clear that there are echoed sections in the thoughts and beliefs around CCC between Nadiya and Vera, despite them being employed at different facilities and having different roles. In order to further explicate the similarities and differences between the two narratives, a second level comparative analysis was completed, the result of which are presented in this section. I also include analysis of the participants' personalities as they relate to the context of their narratives, along with personal reflection of my interpersonal relationships with Nadia and Vera. Lastly, emerging from the comparison of the participants' personalities is a shared understanding of cultural insightfulness; Nadiya's experience with cultural insightfulness is presented first, followed by explanations from Vera's narrative. Cultural insightfulness is introduced as a cumulative outcome of the second level of comparative analysis and later becomes the starting point for the third level of metaphoric analysis presented in Chapter 5.

\section{Echoed Themes}

While both Nadiya and Vera propose their own interpretations on what constitutes $\mathrm{CCC}$, it is the care recipient who is the focus of the care for them both. The only difference is the temporal factor - Nadiya believes that the client's choice should be respected since it has become a habit over the course of the individual's life, while Vera holds that the current needs of the residents are generally associated with the basic requirements of all representatives of the specific age group. Nadiya, therefore, refers to $\mathrm{CCC}$ as keeping the golden middle, which means respecting all those elements that have 
shaped the person into who they are currently. Vera meanwhile refers to CCC as supporting the livinghood of the individual, whereas livinghood refers to the basic needs of the age group along with the deep understanding of how the client's needs are shaped by culture. Although the labels Nadiya and Vera ascribe to the phenomenon of CCC are unique in their own way, the central focus on the resident's needs that are far beyond the physical wellness equate the two in their meaning. Similar among the participants is also their identification of the $\mathrm{LTCH}$ as the Ukrainian environment in which CCC can occur, as well as the attributes of the $\mathrm{CCN}$, which include the ability to listen, be empathetic and open-minded. The themes emerging from this third level of comparative analysis will be detailed further in Chapter 5, where they are integrated into a graphic representation.

\section{Participants \& Personalities: The Context of the Narrative}

Inferences about the participants' personalities are related to the relationship between them and the researcher (Lieblich et al., 1998). Here, I present examples of the interactions between myself and the participants, as well as the emotions evoked, as a way of describing the personalities of Nadiya and Vera. Participant One, Nadiya, is a precise and conscientious person; her emotive self was evoked during the interviews as she was focused on making sure that everything was done just right. For example, Nadiya had taken multiple photographs in an effort to make certain that the photographs convey precisely the intended meanings. Yet, her photographs were problematic for Nadiya, because she did not feel that they revealed the deeper essence of CCC as some of them were dim and distorted. There was also an instance during the third interview where Nadiya became momentarily angry with me when I had asked if the residents "dictate" the kind of care they prefer. Nadiya corrected me in a firm manner, emphasizing that the 
residents make a choice about their needs, rather than dictate their preferences. The exchange between myself and Nadiya, along with my inferences, went as follows:

Maryana: Mm-hmm. So it's the residents then who kind of dictate what they want to do...

Nadiya: [She responds a little sternly] Not dictate, maybe they make choice... they just choose...it's their life...it's just, how they are living in a home...it's not their home where they're from... but they still have to do what makes them happy, right?

Maryana: Right...they have the right to choose. Sorry...I didn't mean to say 'dictate' exactly, but I just wanted to say that...I guess it's their life still, even if it's in a different place, it sounds like what you're saying is that, as a nurse, you're there to help them live the way they are used to.

Nadiya: Exactly! [She exclaims loudly in agreement. I feel that this was a moment when she really defended the residents that she cares for by correcting my use of the word 'dictate'. She is focusing on the residents' right to make choices, which I think really reflects how much respect the participant has for these people.]

(Lines 295-309, Interview 3)

By acknowledging my own faulty choice of vocabulary, I was able to bring the conversation back to the equilibrium. From this point on in our conversation, Nadiya's tone of voice and non-verbal behavior was similar to the manner in which she expressed herself throughout the earlier interviews. She had clarified her stance and was reassured that I understood her position when I verbalized my apology. This exchange with Nadiya, I conclude, is reflective of her approach for providing CCC; Nadiya is protective of the residents and, thus, she aims her care at meeting their preferences and their choices. Nadiya is a woman who seems to follow and enacts her beliefs with precision and wanted to ensure that I would capture her position "just so".

Vera appears to be socially outgoing and curious; she has a strong connection to her Ukrainian culture and profound respect for nature, both of which give her strength in her day-to-day life. Vera's loyalism was evident in her descriptions throughout the 
interviews, where she emphasized the distinct resilience of Ukrainian people and the

practical essence of the natural world. Vera drew the connection between these two in our

third interview, as she described the different uses Ukrainians have for dandelions.

Maryana: Here, sometimes people use the dandelion leaves to make salads, but I never heard you can make jam out of it?

Vera: Yes! And it's very good and delicious and rare. [There is genuine excitement in the participant's voice]

Maryana: That's interesting. It's probably a very old tradition, because I never heard about this. [I easily admit the gap in my own understanding as I can sense the participant would like the opportunity to talk more about this practice of making jam our of dandelions]

Vera: It's old, but it's becoming very popular in Ukraine. I actually have recipe how to make it, but I haven't tried yet. You see how Ukrainians, it's nation of creative people. Very talented. [There is pride and reverence in the participant's voice as she speaks.] People here [in Canada], they think this is weed and they just try to get rid and spray them dead, but somebody took it and made something healthy and delicious from it. That's why, like I tell you about Holodomor [Famine/Genocide], it was a terrible time, but our nation survived. And it's the same with these residents we have. Think...you know how terrible they life was, they... many ran away from home back when it was war and they have to travel...like those big ships, and go through different ways to get here, but they make a good life here, and not just for their own families, but these schools they build for little kids and ...you see how many churches we have? It keeps our community so strong.

(Lines 205-227, Interview 3).

As I got to know Vera's personality closer through our dialogues, I came to realizations that it was important for me to show her my own interest in the Ukrainian community and the Ukrainian "way of life". Injecting moments of personal interest into our conversations led to a more dynamic and sincere interactions. I was also able to acknowledge Vera's understanding of Ukrainian culture as having relevance to our dialogue, which further deepened the level of subjective disclosure of the participant with respect to her experiences of providing CCC. 
Considering the interpersonal context of the data, according to Lieblich et al. (1998), also means including the researcher's reflections. From the perspective of a researcher, my feelings about the incident with Nadiya were aligned with the concern of offending the participant as a person and as a professional. When Nadiya had corrected me, I immediately recognized my own error of insulting the participant's worldview, as well as her professional care. From the tone of her voice, I inferred that she was angry that I did not understand her perspective and was adamant about correcting me. I felt that I had wronged Nadiya, and so I acknowledged my oversight without delay. I was relieved that this cleared the air and the participant was able to carry on with the interview in a relaxed and calm manner. As the style of Nadiya's narrative following the exchange around my use of the word 'dictate' was not altered or negative skewed, I have come to understand that Nadiya had felt comfortable with me, as she was able to provide me with a correction.

When interacting with Vera, I came to realize the boundaries of my own knowledge of Ukrainian culture in comparison to the understanding that somebody who is older may have; in asking Vera to provide me with explanations of cultural traditions, I was restructuring my own awareness of what it means to be Ukrainian. Furthermore, I was creating the opportunity for the participant to draw comparisons between the ways through which Ukrainian culture is expressed by natives in Ukraine versus how it is lived by Ukrainian immigrants in Canada. I feel that I had also subjected myself to the Ukrainian way of being by accepting that I can learn from the wisdom of the older generation. 
Although from the onset, I understood that my own familiarity with the Ukrainian culture and my professional experience as a nurse, could color my interpretation of the narratives, I kept a journal in which I would reflect on my own standpoint. This is how I came to recognize that I am a conscientious individual. This was reflective in my approach towards the participants. For instance, prior to start of the data collection, I had given considerable thought to how I would address the participants. I had settled on referring to the participants as "Pani", which is the English equivalent of 'ma'am', and is a polite way to address those whom you hold respect for. Just as people's relationships differ from one individual to the next, there is an element of variability between the relationships a researcher forms with the different participants. While I would characterized my relationship with Nadiya as being highly professional and equal in status, I had the distinct feeling of being a young trainee, almost like a grandchild who needs to be taught how to be Ukrainian, in my interactions with Vera. The researcher, however, should refrain from allowing the degree of amiability of the relationship affect the interpretation of the narrative data (Leblich et al., 1998). By including the examples of my interactions with the participants, as well as my reflections on them in this writing, I am demonstrating the process of reflection that I had engaged in as I made inferences about the narrative data. This is my way of maintain transparency of the data analysis process.

\section{The Shadows of Their Personalities}

There are some pronounced differences in the narratives of the two participants. Vera devotes a significant portion of her narrative reflecting on the efforts of her colleagues, identifying them as a crucial pillar in her ability to provide CCC. For 
example, Vera's colleagues have helped her settle an agitated resident with dementia by sharing the knowledge of the woman's past preference with Vera. Vera recognizes how effective teamwork has bolstered her ability to provide $\mathrm{CCC}$, and has taken on the responsibility for sharing her knowledge with younger staff. Nadiya also mentions the support of colleagues, but this is done in passing. Instead, Nadiya zeroes in on her own capacity to provide CCC, considering a shared cultural background between herself and the residents as the foundation of CCC.

Perhaps the difference in opinions between Nadiya and Vera can be attributed to the values of the participants' respective workplaces, although this inference can only be based on the descriptions of the LTCHs provided by Nadiya and Vera. It was not the intent of this study to describe the policies and practices of the various Ukrainian-specific LTCHs in the GTA. Still, having come to appreciate the sharp contrasts in the personalities and characters of the two participants, I feel comfortable suggesting that the difference in their reliance on colleagues may be associated with their distinct personalities. Vera's gregarious backbone stands in great contrast to Nadiya's stoicism; this gives a clue to the varying preferences between the two participants.

Vera, for instance, consistently uses the pronoun "we" in her speech, when describing examples from her work. The listener gets the sense that Vera is never alone in her clinical actions; there is usually somebody she may call upon. For instance, she says, "we advocate for our residents", which indicates that there is collective bond between the employees of the LTCHs (Lines 623-624, Interview 3). Vera offers a few more examples of her gregarious personality throughout our discussion; for instance, she describes how she sometimes acts as the liaison between the residents and family members, encouraging 
the "families to take them [the residents] home for the holidays, or include them in their life" (Lines 624-625, Interview 3). Since Vera explains that she "would never leave [the LTCH]", her devotion shows that the collaborative mentality is in line with Vera's personality (Line 433, Interview 3). Nadiya seems to have a more individualistic outlook. For example, when I had elaborated on one of Nadiya's points about the importance of maintaining the cultural feel of the LTCH by linking it to scholarly literature on better health outcomes of older adults in familiar environments, Nadiya answered, "You are telling me from literature, but I know from experience!" (Line 418, Interview 3). During that same interview I had brought up a concept from literature once more referring to "linguistic congruency" as being of importance to CCC. Nadiya reminded me without hesitation, "I say it before. You read literature, but I tell you from my work...from my experience, so it's every day...I see this all the time" (Lines 675-676, Interview 3). Nadiya is confident in her knowledge of Ukrainian culture and her abilities as a CCN, and the care she delivers is very by-the-book. These quotes exemplify Nadiya's stoicism I mentioned earlier. In a way unique to their personalities, the women's narratives overlap on a point about the significance of a deep and personal understanding with the Ukrainian culture. This theme is explored below.

\section{Cultural Insightfulness}

There is a theme which came through in conversations with both participants that was hard to identify at first, but with time, it became apparent that the notion linking the narratives of Nadiya and Vera is the existence of a deeper understanding of culture. I have labeled this emerging concept as 'Cultural Insightfulness'. The ability to make evaluations, and subsequently enact actions based on those evaluations, demands a 
certain level of insight, or deep understanding, it seems. Cultural insightfulness, it seems, for these women is dependent upon the fact that they are both Ukrainian born individuals who grew up in the culture, which links back to the ideas introduced by Stuart and Nielsen (2011) on the introspective understanding of cultural norms. Through their narratives, the participants refer to the elements of cultural insight. Nadiya describes how she was able to rely on her extensive understanding of the Ukrainian cultural traditions, deep and rich because of her own Ukrainian heritage, to amend a resident's practice of refusing medication doses, while Vera narrates an incident from her early practice when she omitted to include her cultural understanding into the care of an elderly Ukrainian immigrant.

Nadiya's experience of cultural insightfulness. Nadiya's recollection of how she was able to merge a resident's religious practice with his medication schedule is an exemplar of cultural insightfulness. A new resident, Mr. M, was prescribed medication to be taken with food; this, however, became an issue on Sundays, as Mr. M would not take in food until he had the Holy Communion during a late morning liturgy. It is traditional for followers in the Orthodox Catholic Right to fast from midnight until they receive Holy Communion during the liturgy. As a Ukrainian herself, Nadiya was aware of the significance that the practice of fasting holds in the Ukrainian religious traditions. In fact, as a member of the cultural group, Nadiya herself follows this tenet. Nadiya admits, "I know not eating before communion is very old and very important ritual, and I do that myself, but sometimes in church they say you can have communion if you didn't eat one hour before" (Lines 631-634, Interview 1). Nadiya assessed that delaying the medication could result in a negative health outcome for the resident. However, based on her cultural 
insight, Nadiya was able to understand and accept the resident's decision, although initially, as a HCP, she was frustrated with the client's behavior until she took the time to understand the context behind it. She voiced as much by saying, "I understood immediately...I don't know why I missed that point, but I understood what he's referring for. Like you know, in our religion, we can't eat until we have Holy Communion. And it's very big deal" (Lines 615-618, Interview 1). She then decided to include the priest in the client's care, who gave the resident permission to have breakfast as long as he would fast for one-hour prior to receiving the Holy Communion.

In explaining the decision-making process, Nadiya made reference to her familiarity with the historical context of Ukrainian people. "In the olden times" Ukrainians living in small villages would rely on either the doctor or the priest in moments of illness. The two authority figures still hold great power during the present day among the older generation of Ukrainians, even in Canada. The priests of the Ukrainian community in the GTA have made themselves available to the Ukrainian nursing homes, frequently visiting the sites to have conversations with the residents. She assessed that the resident has strong spiritual affiliations, which were guiding his decisions. She explains that as a "strong believer of a higher power", the resident "probably would listen to what the priest says", more than to the doctor's recommendations (Nadiya, Lines 210-211, Interview 2). Nadiya also attributes her ability to make the right decision to the fact that she shares the same cultural background as Mr. M. Nadiya notes, “maybe if I wasn't Ukrainian, I wouldn't understand why he didn't want to take the medication. Maybe even not as much as not to understand as I would not accept that" (Lines 661-663, Interview 1). She explains further, "I knew that avoiding 
food before communion is part of our religious traditions. That's what I do...I would really offend him if I didn't let him do what he believed was the right things for him to do" (Lines 663-665, Interview 1). Holding personal knowledge of the cultural traditions assisted Nadiya in developing a plan of action that was respectful of the resident's preferences.

Collectively, this cultural knowledge fused with her clinical and personal assessment skills led Nadiya to identify the priest, rather than the physician, as the most appropriate source of guidance in that moment of care. These decisions may seem simple and effortless on the surface, but are examples of CCC tailored to the client's needs. Nadiya supports this conclusion by saying, "I don't even know [how I decided to call the priest]...I don't think long...I just do it” (Lines 216-217, Interview 2).

Vera's experience. In her narrative, Vera shares a personal recollection of a negative incident where her actions had been guided by common practice, rather than by cultural insight. As a new hire, Vera had noticed during one of her nightly rounds that a female resident was having a hard time falling asleep. While adjusting her pillow, Vera came across a dry piece of bread and deduced that the crumbs were making it difficult for the resident to fall asleep. Her intuition told her that clearing the breadcrumbs would allow the resident to sleep more soundly. Vera recalls her though process at the time of the incident, "I try to fix her bed, but when I put hand under the pillow I feel something...like little dots...it's a bread crumbs...I think to myself No wonder she have hard time sleeping, she's uncomfortable...then I put my hand back and...I feel piece of dry bread...so I take it out and wipe crumbs off the bed... and I ask resident and she said she's okay... and that was it...I go back to my work..." (Lines 298-303, Interview 1). The 
resident was later found in distress, and Vera learned from her colleagues that the piece of bread actually held great personal meaning to the resident, as she had been among the survivors of the Famine/Genocide. Vera notes, "I read so much about Holodomor [Famine/Genocide], but I don't think it affect people I work with" (Lines 327-328, Interview 1). Although she was aware of this moment in Ukraine's history, Vera had acted out of instinct to clear the bread, without giving thought to its significance for the resident. Vera reflects on the importance of having a personal understanding of the history of the people she cares for, noting, "it's important to know habits...but also know some history of those residents... and history of their country" (Lines 308-309, Interview 1). As a result of this incident, Vera has come to rely on her personal understanding and cultural insight in her care. She continues to look for opportunities to further her understanding of the residents' experiences, as Vera notes, "I learn so much history about my country from these people" (Line 351, Interview 1). The example Vera provides in her narrative brings to light how lacking cultural insight may lead to negative experiences for the residents and the $\mathrm{CCN}$, and reduce the likelihood that $\mathrm{CCC}$ will occur.

\section{Chapter Summary}

This chapter presented the findings of two levels of data analysis. First, the inferences emerging from categorical-content analysis were presented according to the narratives of Nadiya and Vera, which answers the question how do nurses employed in Ukrainian-specific LTCHs conceptualize and deliver CCC to elderly immigrant adults?. Both participants refer to the attributes the CCNs, the cultural environment of the organization and the shared cultural understanding with the clients as features that 
promote the delivery of CCC. Additionally, while Vera identifies collegial support as another pillar of CCC, Nadiya reflects on the lack of time, and the related staffing shortage, as barriers to CCC. Next, the results of a comparative analysis are described contrasting the two narratives in order to identify core elements that promote the delivery of CCC. In the following chapter, the outcomes of a third level of analysis will be presented using a visual format, then critically examined. 


\section{CHAPTER 5}

\section{Discussion}

To the best of my knowledge, this study is the first to examine the experiences of nurses of Ukrainian background with providing CCC to elderly Ukrainian immigrant residents in the GTA's Ukrainian-specific LTCHs. The intent of this chapter is to reflect upon the findings of the third level of analysis of the narratives of Nadiya and Vera with respect to the current state of literature and to identify the gaps in the emergent understandings that have implications for future research. A visual representation integrating the major themes is introduced at the onset of the chapter to guide the reader's understanding of the converging ideas of the participants' narratives. The themes include a) honoring the resident, b) home is where the varenyky are served, c) the CCN as the doorway to CCC and d) cultural insight as the solid foundation. Towards the end of the chapter, the researcher's concluding remarks are presented, along with study strengths and limitations. Additionally, implications for practice and policy, along with further research directions are introduced.

\section{Integrating the Findings: A Visual Depiction}

In keeping with the creative slant of this study, I have chosen to represent the major themes that have been woven through the narratives of Nadiya and Vera in a graphic form. The visual depiction, presented in Figure 11, is an organic representation of the themes of the third level of analysis, the metaphorical analysis. The representation speaks to both how the participants conceptualize CCC and how they deliver it to Ukrainian elderly immigrants. The study highlights that cultural insight, nurse's attributes and the cultural environment of the healthcare facility are all essential to the delivery of 
CCC, which is conceptualized as meeting the culturally influenced needs of the care recipients.

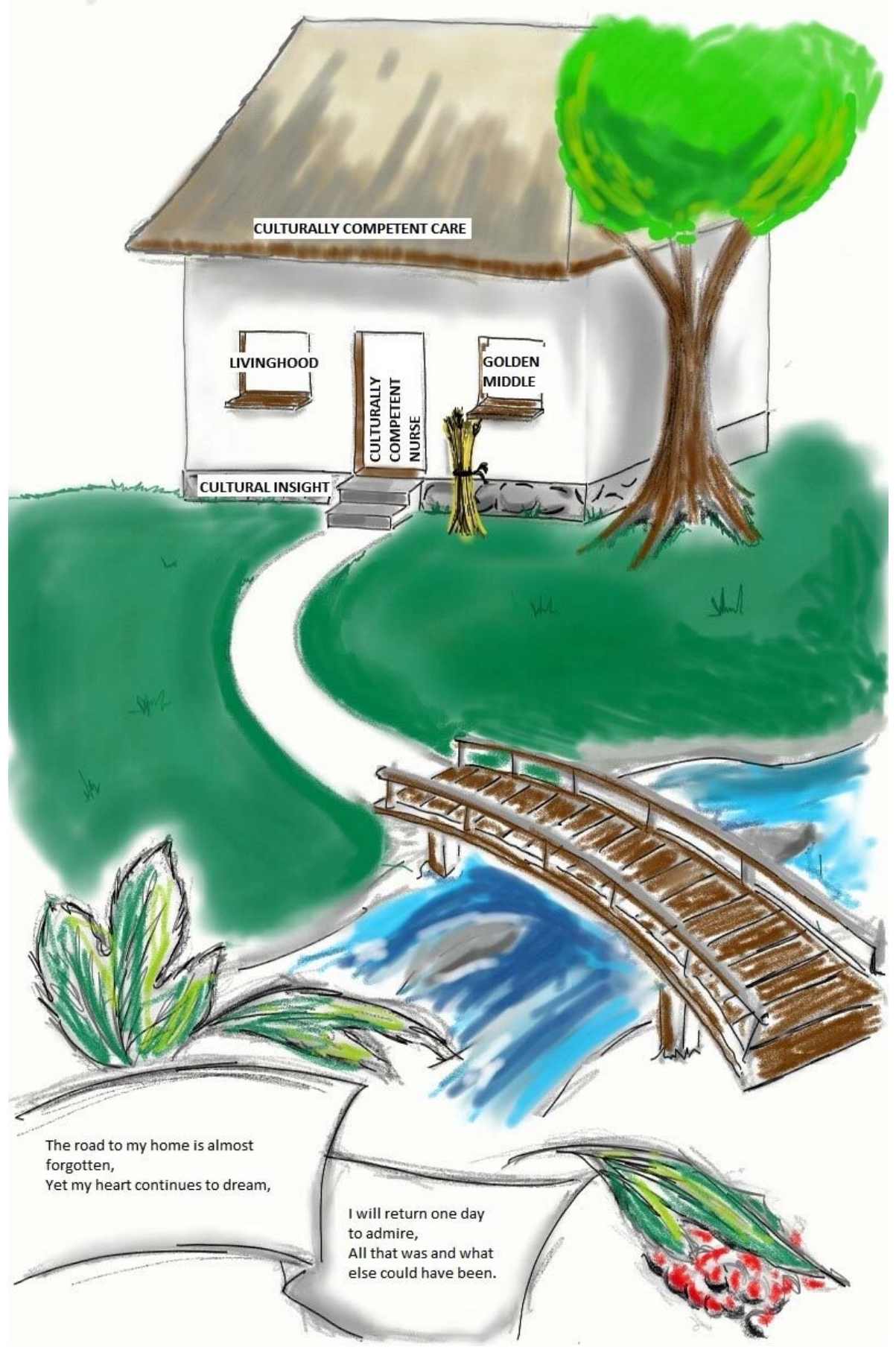

Figure 11: Culturally Component Care in Mono-culture Health Care Facilities 


\section{The Graphic Explained}

The visual depiction of the four major themes that have emerged through the third level metaphorical analysis of the narratives of Nadiya and Vera is largely symbolic, rather than descriptive in its meaning. The depicted house and scenery mirror a typical home in a remote village of Ukraine. The house itself is fairly simplistic from the outside. As was common in the old days the house is a large single room on the inside, where the whole family eats, sleeps and celebrates. A person can live his whole life in that single place, which makes the home a very important constant of people's lives. As Nadiya and Vera explain, it is important to promote the homelike environment of the LTCH to promote the residents' comfort. The roof of the house in the graphic is thatched, having been made of intricately woven hay. I can imagine whole families working together in the field, cutting the hay, waiting for it to dry so that they can protect their home from the elements. Prominent symbols of Ukrainian culture are also included in the graphic, including a bouquet of wheat, called didukh, and the guilder rose, or kalyna. They are part of holiday celebrations in Ukraine. For example, the didukh is brought into the house by the head of the family, a father or a grandfather, and is set at the table on Christmas Eve. It represents abundance of harvest and the spirits of ancestors.

The house that appears in the center of the image represents the setting where CCC takes place. Both participants had spoken extensively about the features of the LTCHs that promote the feel of a Ukrainian home. The house, as it would in real life, sits on a solid foundation, which in this case, represents cultural insight. As I had alluded to in the Findings chapter, cultural insight is at the heart of all interactions between the clients and the nurses; it is a deep and permeating understanding of the Ukrainian culture that comes through the participants' extensive and personal experience with the cultural 
elements. The stairs symbolically represent the notion that the journey of gaining cultural insight is ongoing; there is always the opportunity to learn more, to keep going up.

There is a brief prose that I wrote about the significance of a home as a place of familiarity; no matter where we end up in life, there is nothing quite as comforting as that feeling of coming home. Sometimes our home is not where our current address is, but it is a place that holds personal meaning to us - it is a place we often long for. The LTCH is meant to become the home-away-from-home for the residents, where they receive CCC from a CCN whose knowledge is grounded in personal experience and deep understanding that results in cultural insight.

The bridge that appears in the forefront of the image represents the journey an individual takes though their life - this can include both the nurse and the resident; it is slightly curved and irregular to represent all those instances when individuals interact closely with culture and their understanding of it evolves. When the individuals reach the other side of the bridge, they have a deep and personal knowledge of their culture, yet this understanding may still change in response to what the person encounters upon entering the home. The symbolism of the bridge is also meant to echo the metaphor of the bridge that Vera introduces in her narrative, as the journey into tomorrow for the residents and the collegiality and support of the staff, who stand close together like the pickets along the bridge.

The trees, with their overgrown roots, represent the unique identities of the care recipients. There is reciprocity between one's roots and the culture s/he has grown up in. The $\mathrm{CCN}$ is the door to that house - she is the entryway through which CCC becomes a reality. Depending on how open she is to new learning and different perspectives, she 
may become fully engaged with the clients or she may limit the extent of CCC by opening herself only slightly to the possibility of new learning. And finally, the windows through which one can "see" how to provide CCC represent the concepts of the golden middle and livinghood. By accepting the viewpoints of Nadiya and Vera that place the care recipient at the center of all $\mathrm{CCC}$ activities, the $\mathrm{CCN}$ recognizes the value of promoting the client's livinghood and directs her efforts at keeping the goal of sustaining the golden middle in mind as she delivers her care.

\section{Exploring the Major Themes}

In the pages that follow I present a discussion of the four major themes identified from Nadiya and Vera's narratives, including a) honoring the resident, b) home is where the varenyky are served, c) the CCN as the doorway to CCC and d) cultural insight as the solid foundation. The concept of livinghood is explored under the theme of honoring the resident, with specific focus on the intersection of the findings with the writings of Tom Kitwood (1997). The discussion of the second major theme home is where the varenyky are served includes consideration of the cultural and professional environments of LTCHs, and the use of ethnic language at the facilities. The participants' narratives related to the characteristics of a $\mathrm{CCN}$ are considered with respect to the attributes of cultural competence outlined in Campinha-Bacote's (2002) model. The section concludes with the discussion of the theme cultural insight as the solid foundation, which explicates the grey areas of understanding of how cultural insight fits into the delivery of CCC.

\section{Honoring the Resident}

Although both participants had used their own words to explain how they conceptualize $\mathrm{CCC}$, the consistency between the two narratives lies in their focus on the 
recipients of care. This focus is explored under the theme of honoring the resident. According to the participants $\mathrm{CCC}$ is directed by the social and clinical needs of the client, which are reflective of the individual's cultural background, habits and lifestyles. The participants acknowledge that the needs of the clients vary with age, however, to provide care for the elderly immigrants is to honor their cultural background. In Nadiya's care, this means keeping the golden middle as she facilitates the residents' abilities to carry on their lives in a style similar to what they were accustomed to at home, while introducing elements of nursing care into their days in a non-intrusive, yet supportive manner. Vera also agrees that the needs of the residents are at the forefront of nursing care; to achieve this, she advocates for promoting the client's livinghood. In Figure 11, these two views of the participants are symbolized as the windows to the house. By looking through the windows of the golden middle and livinghood, the participants are considering the needs of the residents as the main focus of their care.

Meeting the needs. The word 'need' is synonymous with 'essentials', or the more stern 'demands', and is meant to refer to instances where something is necessary (Merriam-Webster Online, 2015). When used as part of the definition of CCC, meeting the resident's needs implies a great degree of importance and urgency. This definition fits appropriately with the use of the word 'needs' by the participants, for whom the needs of the client are the overriding principle molding the nurses' care. The concept of 'needs' has been discussed by Kitwood (1997) in his work around PCC and dementia care, where he identifies "love" as the all-encompassing need of individuals living with dementia. Kitwood equates the need for love with the overlapping of five other great needs, including comfort, attachment, inclusion, occupation and identity. Kitwood argues that 
fulfilling these five great needs in turn fulfills the person. Although, according to Kitwood, these great needs are more pronounced in persons with dementia, he acknowledges that these needs are experienced by all people while the extent of needs usually varies among people.

In their narratives, Nadiya and Vera speak to moments in their CCC of older immigrant adults where they fulfill the great needs proposed by Kitwood (1997). Comfort is the freedom from pain, constraint or distress; to promote comfort is to provide warmth and strength that helps the individual overcome difficulties (Kitwood, 1997). Clients are likely to experience feelings of comfort and trust in care provider when they receive CCC (Tucker et al., 2011), which furthers their satisfaction with their HCPs overall (Sorensen et al., 2005) and enhances their sense of personal control of the care (Jahng, Martin, Golin, \& Dimatteo, 2004). Vera lists comfort as one of the needs that are to be satisfied by a CCN through CCC. As the LTCH is often the individual's final place of residents, CCNs strive to promote comfort for the residents. Attachment, or the forming of a bond, is another need referenced in Vera's description of how the nurses attend funeral masses for the deceased residents with whom they have formed a bond similar to the devotion that blossoms between family members. Nadiya's narratives, meanwhile, reflect on the residents' needs for occupation, or the engagement of the individual's abilities in a meaningful way. When Nadiya explains that the aim of CCC is to help the residents maintain their habits and lifestyle, she speaks to the need of occupation as defined by Kitwood (1997). In her capacity as the CCN, Nadiya creates the opportunity for the residents to participate in the activities familiar to them, such as reading. 
The nurses meet the residents' needs for inclusion, or the feeling of belonging and the opportunity for interactions described by Kitwood (1997), by engaging the residents in meaningful conversations reflective of their past and the shared background. For example, Nadiya narrates her experiences of conversing with a resident who originates from the same geographical region in Ukraine as does she; the two are able to share stories about the mountainous landscape of the area. Further to this, Nadiya can then use the understanding gained through these interactions to provide CCC that keeps the golden middle by avoiding extreme clinical actions. For instance, through the resident's stories, Nadiya assesses the level of mobility and pain, and is able to include this information in her care. Through such dialogues, and by incorporating elements of the Ukrainian culture into the care she provides, the $\mathrm{CCN}$ promotes the residents' identity, the final great need identified by Kitwood. At the core of the interactions between the participants and the residents of the Ukrainian LTCHs is the shared identity of being Ukrainian. The nurses, therefore, base their CCC around the common elements associated with the Ukrainian culture.

The concept of 'needs', according to Kitwood (1997), is "always expressed within a particular framework, and thus impregnated with that culture's meanings and values" (p. 81). In meeting the needs of the residents, the $\mathrm{CCNs}$ demonstrate respect for the cultural undertones that shape the residents' lives. The importance of incorporating cultural traditions into the care of older immigrant adults as a means of meeting their healthcare needs has been emphasized in the Canadian-based study by Mullings and Gien (2013), who had set out to explore the potential gaps in the availability of culturally appropriate healthcare and LTC services for older immigrants in the country's Eastern 
region. A similar concept of orienting care according to the needs of the clients was noted by Fuller (2003), who conducted an interpretive study, interviewing 20 Canadian health service informants and a multicultural coordinator from a teaching hospital in Australia, with the aim of furthering the understanding of how to provide CCC to immigrants in a multicultural population. The Canadian informants of Fuller's study agreed that, in intercultural care, it is essential to ask clients questions to understand their needs, and then to use the provided information to shape the direction of care. Cortis and Kendrick (2003) note that nurses are ethically obligated to care for the needs and expectations of their clients. This is reflective of the principles of individualized care, which is associated with positive health outcomes (Suhonen et al., 2005). In meeting the needs of the residents, Nadiya emphasizes the importance of keeping the golden middle, as a way of maintaining balance and addressing all the needs in a timely and respectful manner.

Understanding livinghood. HCPs acknowledgement of personhood is a core element of PCC. Yet Kitwood's (1997) conceptualization of the role that cultural practices play in forming personhood could be strengthened, particularly given more recent literature supports that cultural practices and beliefs are inherent to identity. When cultural practices and background are explicated to a greater degree than is present in Kitwood's emphasis on life history, as the narrative emergent from the findings of this thesis suggest, the phenomenon of livinghood materializes. Promoting a resident's livinghood means caring for the person's culturally-specific age-related needs, along with demonstrating respect and understanding towards the individual's cultural roots. On the surface this definition reflects the ideas associated with Kitwood's (1997) concept of personhood, yet, upon deeper examination, distinctions can be hypothesized between the 
two. Kitwood explained how personhood recognizes that value is bestowed upon one person by another. Essentially, personhood is granted almost in a paternalistic way by the nurse to the individual who is living in the LTCH, particularly if they have a cognitive impairment or a physical disability that requires extensive interventions from the staff (Kitwood, 1997). The individual is recognized as having value that does not diminish due to age, illness or social status. Livinghood, however, seems to have something else to it; inherent to this concept is a reciprocity of understanding between the nurse and the client grounded in cultural respect and the foundational grounding of cultural insight. This value is closely associated with the HCP-recipient's shared cultural background, therefore, in caring for the person, a $\mathrm{CCN}$ introduces elements of that person's culture into the care because they understand it on a deeply personal and experiential level.

There is overlap between the related concepts of PCC, personhood and livinghood, which warrants further study to understand whether livinghood is a distinct concept or if it is an extension of personhood in some capacity. The uniqueness of livinghood seems to lie in its insightful way of understanding the other person as not only a human being whose value comes from them being a person as does personhood, but as an individual whose outlook is a reflection of direct experience with a particular culture. While PCC does focus on culture as an element of one's being, personhood, which itself has been born out of PCC, side steps the distinct phenomenon of cultural identity through its more general focus on the value of one's "humanity" (Kitwood, 1997). As was mentioned earlier, further concept analysis work can help make the distinction between cultural insight and livinghood emergent from my study, along with earlier work on such 
concepts as cultural proficiency (Cross et al., 1989). These concepts may be related, however, it is not yet known whether they are necessary conditions for CCC and/or PCC.

The resident is the axle around which all care revolves. It is the aim of CCC to support the resident's livinghood, explains Vera. Livinghood is the amalgamation of the heritage and the cultural background of the individual. The concept of livinghood, therefore, appears to go beyond the tenets of personhood and PCC, as it captures additional elements that have not been considered in association with the clients' cultural needs by personhood or PCC. Through personhood, Kitwood (1997) focuses more on what it means to be an aged "human" living with an intact identity despite severe disability. Livinghood reflects cultural and ethnic pride. However, the notion of livinghood is emergent. Further research involving larger sample sizes and across more diverse ethnic groups, as well as further concept analysis will be required. What can be said now about livinghood is that it is an important concept associated with CCC, in particular in mono-ethnic LTCHs where there is cultural similarity between the care recipient and some of the care providers. This opens the door to potential future research directions, which are discussed in the section Implications for Research.

\section{Home is Where the Varenyky Are Served}

While the initial purpose of the study was to understand the experiences of nurses employed at a mono-ethnic $\mathrm{LTCH}$, the healthcare setting was intended to act as a reference point for the participant inclusion criteria. The cultural atmosphere of the LTCH, however, was identified by Nadiya and Vera as an integral attribute related to their ability to provide CCC. Although wait times for admission to culturally-specific LTCHs are typically longer, the designation of the LTC facility as a Ukrainian 
environment commonly attracts residents of Ukrainian heritage (Community Care Access Centre, 2015). As a culturally-specific LTCH, the facility may promote the cultural environment though the presence of traditional artifacts, ethnic foods and use of the ethnic language (Barrass, 2012; Hikoyeda \& Wallace, 2001).

The participants described an extensive list of traditional Ukrainian features of the LTCHs where they are employed, such as displayed embroidery, decorated pysanku (Easter eggs), and Ukrainian literature, including books. Collectively, the presence of these items gives the LTCH a Ukrainian 'feel', explain the participants, which is further promoted in the architecture of the facilities. For example, Vera makes reference to the use of natural wood as a décor element of the home, which is reminiscent of the common use of wood in a Ukrainian home. Additionally, the LTCHs respect the residents' religious practices, and provide space for worship, such as a chapel. The availability of Ukrainian ethnic foods in the residents' diet, along with dishes that are part of holiday customs, further promotes the Ukrainian culture within the home.

The participants explain that providing CCC to Ukrainian residents is facilitated by the Ukrainian environment of the home, since they can include the traditional elements familiar to the residents into their care. The value of a homelike environment is supported by the principles of PCC (Caspar et al., 2009). It can also be inferred from the narratives of the participants that the Ukrainian character of the home is important to the residents as a means of preserving their cultural lifestyles. Yeboah, Bowers and Rolls (2013) emphasize that culturally and linguistically diverse older adults bring their culture with them upon relocation to LTC. Their conclusion is based on a study consistent with the grounded theory method of the experiences of 20 residents of four nursing home in 
Australia. According to Yeboah and colleagues, the elderly immigrants "continue to weave their cultures of origin into their lives, through food, music and language even after relocating to a new environment that lacks the necessary support to do so" (p. 59). It seems fitting then, that facilities which are ethno-specific preserve the culture of the residents through symbolic features, foods and language. As Mullings and Gien (2013) have uncovered, even for those individuals who self-identify as being integrated into the Canadian culture, the need for cultural elements in their care persists; lack of such cultural aspects is associated with feelings of exclusion and isolation, which are further compounded among the older immigrants who are residing in LTCH that is oriented at promoting the dominant cultural perspective of the West. Feelings of social integration, however, are more likely to develop among older immigrants who are residing in a culturally homogeneous LTC environment, as was explained by Wister, Pereira, and Lazarowich (1996), whose study had focused on Toronto seniors of Italian and Portuguese descent living in either culturally homogeneous or heterogeneous LTCHs in Toronto.

The ability of an ethno-specific LTCH to meet the cultural needs of the residents, including the presence of ethnic food, is viewed positive by the family members of residents (Runci, Eppingstall, van der Pioeg \& O’Conner, 2014). Although Hikoyeda and Wallace (2001) found an elevated preference among family members of older Japanese immigrant women for LTCHs that mirror the cultural elements of a traditional Japanese home, the colleagues note that the "ethic-specific environment alone was insufficient in meeting all the resident's needs" (p. 100). This indicates that other factors affect clients' experiences, including the behavior and attitudes of healthcare staff, which are explored 
under the theme CCN as the Doorway to CCC (Hikoyeda \& Wallance, 2001). It is interesting to also note that availability of ethnic food is among the main considerations of families when deciding on a LTCH. The older Japanese American immigrant women in Hikoyeda and Wallace's study who were residents of a culturally-heterogeneous LTCH would tolerate the routine diet, but had expressed that they would prefer ethnic food. A consensus on the significance of food was also reached among the 24 informants of Gorek et al.'s (2002) study about the culturally-guided preference for placing older Latino family members into LTCHs. Facilities that did not offer authentic Mexican food were viewed as demonstrating disregard for the Latino heritage of the elderly residents (Gorek et al., 2002). Aside from its value as sustenance, ethnic food is essential for its ritual and traditional connotations, and can therefore positively contribute to the cultural feel of the LTCHs (Barrass, 2012).

While there is some mixed literature related to the experiences and perceptions of quality of care at ethno-specific LTCHs from the perspective of family members, residents and HCPs, it is a challenge to locate literature that explores whether the cultural feel of the home is a factor in the nurse's ability to provide CCC. The overall paucity of literature on ethno-specific LTCHs can, at least partially, be explained by the diminished preference for institutionalization among many ethnic groups (Barrass, 2012; Disman \& Disman, 1995). For example, while older immigrants make up close to $11 \%$ of the population of Chinese Canadians, it is culturally expected that the children and extended family will provide care for the elderly (Chau \& Lai, 2011; Lai, 2002). Although the perceptions have been shifting towards the Western norms in recent times, 
institutionalized care, to some extent, is still a taboo topic among many cultural groups (Barrass, 2012).

There are some scholarly proponents of the mainstream approach to care who do not give preference to the ethno-specific LTC facilities. For instance, Petrov and Arnold (2000) write that care settings that operate with reference to models such as PCC are able to provide quality care to all individuals. Jones (2011) also maintains that a healthcare environment devoted to the quality of care that adapts the PCC outlook can enhance the quality of life for the residents. It is possible that a healthcare environment based on the principles of PCC may impact both the care recipients and providers. The care providers become more engaged with the clients, which creates a sense of empowerment among both groups; further to this, the individuality and the opportunity to make choices among the residents is respected, as they are treated with dignity (Robinson \& Gallagher, 2008; Tellis-Nayak, 2007). The residents of PCC organizations interact with the staff on a frequent basis and in meaningful ways, which can enrich their lives, and transform the nature of the client-provider relationships into a friendship (Gnaedinger, 2003). Brotman (2003), however, disagrees with this viewpoint, noting that the multicultural approach of the mainstream LTC is insufficient to overcome the racism that culturally diverse residents encounter in their care. Although already an improvement over the medical model of institutional care, PCC reflects on the importance of culture to the client's wellbeing only marginally. The main focus of PCC is on promoting autonomy and the client's right to make choices (Kitwood, 1997; Jones, 2008; Robinson \& Gallagher, 2008), yet Tayab (2015) has found that LTCH staff, in particular the PSW participants of her study, often lack access to the information about the residents' unique needs for various reasons, 
including lack of time. The findings of this study related to the significance of the cultural environment of the LTCH as an element of CCC highlight the importance of incorporating physical manifestations of culture into the care of culturally diverse client groups.

Ukrainian language. Among the cultural features of the LTCHs' environments described by the participants is also the predominance of Ukrainian language. According to the participants, the staff of their respective LTCHs commonly speak in Ukrainian amongst themselves and when addressing the residents, although the documentation is done in English. Communication is central to CCC, as it allows for the exchange of information between the nurse and the client, explain the participants, and that can then be used to direct the nurses' care. The participants of Gunaratnam's (2008) study, including 56 health and social care professionals, agree that holistic care, which includes an emphasis on cultural needs of the client, is born out of knowledge and understanding of such needs. The knowledge and understanding, however, is best derived from direct interactions with clients, rather than reliance on general understanding of the cultural group (Gunaratnam, 2008). It is through communication that the nurse learns about the client's needs and preferences; CCC is grounded in this understanding, explain Nadiya and Vera. CCC is built on communication, and the resulting companionship between the residents and HCPs (Heikkila \& Ekman, 2003).

Language incongruence, and the related struggle with communication, is acknowledged in the literature as a significant barrier to providing CCC (Coffman et al., 2004; Regev, 2014; Star \& Wallace, 2009), in particular in LTC (Mulllings \& Gien, 2013). As Hearnden (2008) points out, communication in the realm of healthcare is 
already a complex issues, since it often occurs in stressful circumstances, but it is further complicated by language disparity between client and recipient. This issue is even further intensified in the care of older adults, who, as Mullings and Gien (2013) point out, may revert to speaking their native language with advance age. The participants of the current study reflect on the importance of sharing a language with older Ukrainian immigrants who have lost their knowledge of English, either due to illness or advance age. By speaking in Ukrainian to these individuals the nurses foster understanding with the resident, and create a more positive relationship. In the LTC environment overall, language is important as a means of promoting the resident's socialization and for identifying a resident who is in distress, or as part of diagnosis and treatment; it is, as Barrass (2012) explains, needed for connection, health and welfare.

Difficulty communicating due to language differences has been associated with feelings of frustration for both the care recipients and the care providers (Regev, 2014; Ulrey \& Amason, 2001). Nadiya and Vera spoke of their English-speaking colleagues' interactions with Ukrainian-speaking residents, narrating the mutual feelings of frustration that emerge. Nurses feel that they are not able to provide as high quality of CCC to linguistically diverse individuals as they would to those with whom they share a language (Ulrey \& Amason, 2001). Language incongruence is also viewed unfavorably by family members. For instance, the main objective of members of the Latino families participating in Gorek et al.'s (2002) who had a loved one in LTC was to promote the "normal" lifestyle for the residents, however, they viewed staff in LTC who did not speak the language as a barrier to their ability to promote the resident's comfort. Capell and colleagues (2007), therefore, emphasize that technical proficiency is no longer the sole 
requirement for HCPs as it has been replaced by effective communication skills.

Communication helps reinforce the development of a therapeutic relationship, which promotes understanding and respect between the nurse and the client, and creates the opportunity for the CCN to understand the residents' needs, as explain Nadiya and Vera. This conclusions is confirmed by Hart and Mareno (2013). The participants of their qualitative study of the barriers to CCC, a total of 374 nurses from the US, identified language disparity as a barrier to forming connections with the clients and building trust into the therapeutic relationships. Lange, Mager and Andrews (2013) emphasize that miscommunication and misunderstandings may also be attributed to cultural mismatch between the client and the care provider. This point is also emphasized by Nadiya and Vera, both of whom reflect on the occurrence of misunderstandings between Ukrainianspeaking immigrant residents and English-speaking care providers. Lange and colleagues write "provider insight into the culturally imbedded belief systems of their patients is an integral part of effective communication that can ultimately impact patient outcomes" ( $\mathrm{p}$. 58). To this extent, Hearnden (2008) explains that effective communication means using language that demonstrates understanding of the context of the interactions, as well as the nuances of cultural situation.

Mold et al. (2005) suggest that communication in healthcare can be improved by increasing the recruitment of ethnically diverse groups into the healthcare workforce, however, as Kai (2005), points out, communication is also affected by sociocultural and economic factors, such as age, gender, education, family and religion. These may promote intra-cultural and inter-generational communication challenges despite the shared cultural background of the client and the nurse, resulting in poor quality of care 
(Giles et al., 2003; Harwood, 2007). Bourdeault, Atanackovic, Rahis, and Parpia. (2010) recognize that Canada's ancillary healthcare staff are often immigrants from Asia or the Caribbean, which perpetuates language difficulties between the staff and the residents. Although the participants of the study do not identify the cultural backgrounds of the diverse staff of their LTCHs, it is evident in the data that their cultural backgrounds differ from those of the majority of the residents. This may complicate their ability to communicate with the Ukrainian residents of the LTCHs.

There is support in the literature to the participants' notion that improving availability of CCC means enhancing the language and communication skills of the HCPs (Iecovich, 2000). For instance, multilingual nurses in Hart and Mareno's (2013) study identified their ability to speak another language as a facilitator in their ability to provide CCC. Ulrey and Amason (2001), however, suggest that HCPs who identify themselves as being more culturally sensitive also tend to demonstrate more effective intercultural communication and, thus, report less anxiety during instances of intercultural interactions. Finally, Ponce, Hays and Cunningham (2006) also suggest that older adults' access to better quality healthcare can be improved by offering language assistance services. Although this approach is not without fault. Some HCPs find it challenging to form a therapeutic relationship with their clients through an interpreter (Hart \& Mareno, 2013). Also there is inconsistency in availability of interpreter services in the healthcare system overall (Barrio et al., 2008). Boi (2000) confirms that, from the perspective of nurses, language barriers are further exacerbated by limited availability of interpreters. Overcoming language barriers, therefore, is essential for effective CCC, however, language discrepancy appears to be a system level issue. 
Organizational barriers to CCC. When discussing the LTCHs as the physical setting where CCC occurs, it is also important to consider the professional environment of such facilities, namely the rules, guidelines and regulations, and how they impact on the nurse's ability to provide CCC. The organizational barriers to $\mathrm{CCC}$ evident in the participants' narratives was the lack of training on CCC in their orientation to the LTCHs, as well as lack of time to provide CCC. Hart and Mareno (2013) conclude that lacking resources, including time, money or training, challenges the HCPs' ability to provide CCC. Both Nadiya and Vera remark that they did not receive any formal training related to $\mathrm{CCC}$ as part of their orientation when they were hired by their respective organizations. Lack of knowledge and training related to $\mathrm{CCC}$ has been reported by nurses in Chang et al.'s (2013) study the results of which are based on a cross-sectional survey completed by 230 nurses employed in a specified region of Taiwan. Coffman and colleagues (2004) explain that clients receiving care from nurses lacking experience with and training on $\mathrm{CCC}$ are likely to be on the receiving end of ethnocentric attitudes, misguided communication, inaccurate diagnoses, and ineffective interventions.

Boi (2000) has also noted that issues related to lack of nursing education and training are common in the care of culturally diverse clients; majority of the nurses in Boi's study employed at an urban hospital in the UK felt that they have not been educationally prepared to provide CCC. Some of them, however, did note that their knowledge and understanding of the cultural needs of the clients developed through interactions with the persons, their relatives and colleagues, as well as through reading of research publications. Although, neither Nadiya nor Vera make reference to seeking out professional opportunities for formal education to CCC, they do acknowledge how their 
understanding of the residents' needs commonly evolves through on-the-job learning, experience and interactions with the clients. In Vera's narrative, the support of her colleagues has been instrumental in her ability to provide CCC despite the lack of training and education. Training related to cultural competence may further improve teamwork among care providers (Lange et al., 2013). In terms of learning from experience, Tabari-Khomeiran et al. (2007) explain that nurses learn through direct experience, meaning by doing the work themselves, or through vicarious learning that occurs by observing others engage in the activity. Vera, for instance, narrates her experience of having both the experience of learning through her own actions, as well as by observing the $\mathrm{CCC}$ of her colleagues.

There is a discrepancy in the literature on whether the level of education of nurses is associated with the ability to provide CCC. For instance, Cooper Brathwaite (2006) and Doorenboos and Schim (2004) note a strong positive association between the nurse's ability to provide CCC and her level of education. Schim et al. (2005) report that HCPs with prior cultural competence training and a higher level of educational attainment score higher on the cultural competence behavior scale than their counterparts who lack training and have lower levels of education. This last study is particularly important to consider, since it was conducted with the aim of comparing cultural competence between Ontario and Michigan (US) HCPs. The study was a cross-sectional descriptive design ( $n=71$ Ontario and $n=74$ Michigan). Of the total sample of HCPs from Ontario, nurses made up $71 \%$ of respondents. Similar to these findings, HCPs with higher education levels have been noted to demonstrate higher confidence in their cultural skills in caring for clients of diverse cultural backgrounds by Carson \& Bond (2004). 
Meanwhile, Star and Wallace (2009), in their study of 31 primary healthcare nurses, did not find educational background to be a significant factor associated with the nurse's ability to provide CCC, although this may be due to the homogeneity of the participant group (i.e. Caucasian, bachelor's level of education). Furthermore, there is lack of evidence as to which mode of training would result in sustained ability of HCPs to provide CCC (Star \& Wallace, 2009). Such educational models as workshops (Cooper Brathwaite, 2005; Williams, 2002), immersion in different cultures (Fahrenwald et al., 2001; Walsh \& DeJoseph, 2003), and courses on cultural competence (Crandall et al., 2003) have been shown through research to improve the HCPs' ability to provide CCC. Nurses themselves are commonly in favor of completing training and education initiatives that will aid them in delivering CCC (Boi, 2000; Regev, 2014), yet limited training is offered in LTC settings (Hasson \& Arnetz, 2008).

Factors outside of the nurses' educational preparation and training may impact their ability to provide CCC, including personal characteristics (considered elsewhere in this work) and the mandates, as well as the emotional and physical environment of the healthcare facility (Tabari-Khomeiran et al., 2007). For instance, Tabari-Khomeiran et al. (2007) note that nurses employed in teaching facilities are less likely to develop competence since a large portion of their work is transferred to students. Focusing on organizational barriers to $\mathrm{CCC}$, it is essential to consider the emotional environment of the workplace; for instance, Nadiya identifies lack of time as a barrier to CCC that is the result of staffing shortages at the workplace. The participant reflects that, due to lack of time, she misses out on opportunities to introduce moments of individualized care into her routine, which are essential to CCC. While institutions that increase the nurse's 
rotation schedule in an effort to maintain costs have been noted to present the staff with additional opportunities to develop CCC skills as a result of more frequent interactions with clients, the increased workload associated with this approach may also contribute to feelings of frustration among the HCPs and inhibit their desire to learn (Tabari-Khomeira et al., 2007). Lack of human and financial resources, including time, is, therefore, often identified as a barrier to CCC by the nurses (Star \& Wallance, 2009).

A related perspective on the importance of time for $\mathrm{CCC}$ has been considered in the literature from the perspective of the care recipients. When making a health-related decision, older immigrant adults need time to consider the cultural issues related to the decision, and often rely on their family's involvement in the decision-making process (Giuntoli \& Cattan, 2012). Lack of time due to organizational practices, such as employing a small number of interpreters, may affect a resident's ability to process information; this is of particular relevance to residents who are linguistically diverse (Giuntoli \& Cattan, 2012). It is valuable to emphasize that among the participants $(n=134)$ of Giuntoli and Cattan's study of the experiences and expectations of care and support among older migrants in the United Kingdom were immigrants from Ukraine $(\mathrm{n}=12)$. No specific findings associated with this group of the participants was described by the authors. Limiting the availability of time in the healthcare context, therefore, compromises both the providers' ability to deliver CCC and the residents' decisionmaking experiences, both of which are, in turn, related to decreased quality of care.

\section{The CCN as The Doorway to CCC}

Through their narratives, Nadiya and Vera contribute to the development of a composite of a CCN capable of delivering effective CCC. The nurse is the entry point 
through which CCC emerges. She is, therefore, represented as the door to the house in Figure 11. A CCN, driven by her desire to meet the client's needs and the willingness to learn, builds therapeutic rapport with the client by listening and being attentive to the nuances of shared stories. She wants to understand where they come from and how their roots have shaped their needs. By being open-minded and respectful of client beliefs that differ from her own, the nurse demonstrates regard for the person and his/her background. In her subsequent interactions with the client, the $\mathrm{CCN}$ weaves elements of culture - like the words unique to their region of origin - into her care. Her understanding of clients needs grows with experience, and she continuously demonstrates empathy and understanding.

The joined description provided by Nadiya and Vera is reflective of the personal characteristics, skills and attitudes associated with a CCN in the literature, including open-mindedness, willingness to learn and ability to listen (Boi, 2000; Carey, 2011; Coffman et al., 2004; Fuller, 2003; Leighman, 2004). Clients are more likely to consider a nurse as skillful when she demonstrates strong interpersonal skills while less significance is given to the nurse's technical skills (i.e. operating intravenous pumps) (Wysong \& Driver, 2009). In narrating their experiences with CCC, the study participants focused primarily on interpersonal skills of CCNs, including being a good listener, demonstrating a positive attitude towards work and compassion towards the residents.

In the literature, interpersonal sensitivity towards the client is expected to predominate when the client and the HCP are matched based on cultural background (Pope-Davis et al., 2002). Yet, some researchers have questioned the effectiveness of ethnic match, including Hikoyeda and Wallace (2001), who noted that older Japanese 
American women residing in LTCHs demonstrate preference for HCPs who exhibit strong interpersonal skills, despite their cultural background. Nadiya and Vera have identified specific interpersonal skills as being integral to $\mathrm{CCC}$, however, they believe that sharing a cultural background with their clients adds deeper meaning to their interactions. Cultural insight born out of ethnic match, according to Nadiya and Vera, adds an extra layer of understanding to their relationship with the clients. In their study Heikkila and Wallace (2001) had not considered the perceptions of frontline HCPs regarding the role of ethnic match within their care. Additionally, their participants were only elderly females with family members, whose experience may differ from elderly Japanese American males, as well as other residents with family members. Finally, factors other than ethnic match, including feelings of isolation, may have influenced the participants' care experiences (Heikkila \& Wallace, 2001). It is, therefore, important to continue to study the interplay between ethnic match, attributes of HCPs, and clients' experiences to better understand CCC.

In instances when the HCP's interpersonal sensitivity is lacking, reduced engagement between the care provider and recipient may be noted, along with lower quality of care (Ngo-Metzger, Telfair, Sorkin,Weidmer, Weech-Maldonado, Hurtado et al., 2006). For example, Phillips, Chiriboga and Jang (2012), in their exploration of interpersonal sensitivity as a component of CCC as perceived by a sample of 2075 racially/culturally diverse participants, report that clients are more likely to self-rate their health as being of better quality when they perceive their HCPs as having greater interpersonal sensitivity. Nurses themselves commonly identify interpersonal understanding, information gathering, compassion and responsiveness as being positive 
competencies associated with nursing care (Zhang, Luk, Arthur \& Wong, 2001).

Experience, as well as such personal characteristics as willingness to learn, asking questions and a positive attitude are among the factors affecting competence building of nurses identified by Tabari-Khomeiran et al. (2007).

Applying Campinha-Bacotes' model. In the chapter on literature review, Campinha-Bacote's (2002) PCCDHS model on cultural competence in healthcare was introduced. Nadiya's and Vera's narratives related to the CCN reflect all five of the constructs of the model, although to varying degrees. The construct of cultural awareness is centered around "self-examination and in-depth exploration of one's own cultural and professional background", explains Campinha-Bacote (p. 182), and includes the process of recognizing one's own biases, assumptions and prejudices. Although the participants of my study did not explicate their own experiences with self-reflection around their cultural and professional background, the results of this process are evident in their comments around their knowledge of Ukrainian culture. Additionally, the participants were able to hypothesize that it would take time and effort to learn how to provide CCC to residents of cultural backgrounds distinct from their own. Their narratives, therefore, are an example of a well-developed attribute of cultural awareness. This is an interesting discovery, since the participants, both of whom have been with their mono-ethnic LTC employer for about a decade, have had little opportunity to engage in cross-cultural interactions in their practice.

As Nadiya and Vera describe, the majority of the residents of their LTCHs are of Ukrainian background, yet Campinha-Bacote (2002) explains that through the attribute of cultural encounters, meaning direct interactions with individuals of diverse cultural 
backgrounds, the HCP learns to modify his/her own understanding about other cultures. The participants, however, did demonstrate understanding of the importance of attaining cultural knowledge when caring for culturally diverse clients (i.e. clients from distinct regions of Ukraine). Cultural knowledge refers to the educational foundation for providing CCC, meaning the effort of seeking out opportunities to learn about the healthrelated beliefs and cultural values, disease incidence and treatment efficacy with respect to a cultural group (Campinha-Bacote, 2002). Although they have not received formal training on CCC from their employers, the participants recognize its value. From their narratives, it can be inferred that the participants, if they were given the opportunity, would be willing to participate in CCC training and education with the intent of advancing CCC skills.

Well-developed among the two study participants was also Campinha-Bacote's (2002) attribute of cultural skill, which is the "ability to collect relevant cultural data regarding the client's presenting problem as well as accurately performing a culturally based physical assessment” (p. 182). For instance, Nadiya narrated two examples from her practice where she was able to incorporate her cultural skills to gather information about the residents' well-being and to apply it in a manner favourable to their health outcomes. This includes the earlier-described incidents with Mr. M, the gentleman who had refused to take his medication until he had received the Holy Communion, and the elderly woman who would describe to Nadiya her experiences of ambulating on the hillsides of the mountains familiar to Nadiya. In addition to assessing the residents' physiological well-being, Nadiya was able to effectively incorporate cultural beliefs, values and practices, as well as the past experiences, into the care of her clients. 
The final construct of Campinha-Bacote's (2002) model is cultural desire, which is the HCP's motivation to learn about the different cultures. Inherent in the definition of cultural desire is the HCPs' genuine passion to understand and accept the worldviews of their clients (Campinha-Bacote, 2002). By placing the emphasis on the importance of not only listening to the clients, but also hearing what is said and incorporating it into their care, the study participants had demonstrated their well-developed cultural desire.

In summary, valuable attributes of a CCN include openness (Doorenbos, Schim, Benkert, \& Borse., 2005), a non-judgmental stance and the willingness to deliver care based on others' needs (Gray \& Thomas, 2006). Awareness of one's own cultural beliefs, along with the related ability to recognize the differences between cultural traditions of others, has also been recognized in the literature as a foundational dimension of CCC (Capell et al., 2008; Dudas, 2012; Giger et al., 2007). The participants demonstrate deep knowledge of their own cultures, which is helpful in their efforts to understand the cultural beliefs of others, along with the willingness and the desire to learn (Dudas, 2012).

It should be emphasized that it was not the purpose of this study to determine the extent of the participants' cultural competence or their ability to provide CCC, but rather to narrate their experiences of providing CCC. To some extent, I had anticipated that the participants who came forward would have experience with CCC and want to share their understanding with the researcher. As Dudas (2012) confirms, there is no definitive end point at which a nurse is considered to be fully culturally competent. A nurse develops the ability to provide CCC gradually, with time and through constructive interactions with culturally diverse clients, relying on her skills and attitudes to guide her interactions 
towards understanding the preferences and needs of the clients (Campinha-Bacote, 2002).

In their narratives, the study participants speak to the notion that learning to provide $\mathrm{CCC}$ is an iterative process. Nadiya and Vera recognize that although it may be a challenge to offer CCC to residents of diverse cultural backgrounds, they may rely on past experiences, skills and knowledge to develop a greater understanding of the cultural needs of new clients.

\section{Cultural Insight as the Solid Foundation}

The discussion that follows in this sections focuses on the importance of cultural insight as the foundation upon which CCC is built. Just as the house in Figure 11 is stabilized by its stone footing, the study participants narrate how the CCN's ability to provide CCC is grounded in personal, deep and evolved understanding of the cultural background of the clients. As evident in the findings of this study, one of the factors that contribute to the cultural insight of the $\mathrm{CCN}$ is that she shares a cultural identity with the residents. Based on the collected data, it can be inferred that individuals born into the same culture as their clients are likely to develop cultural insight as they go through life having frequent and extensive interactions with the elements of their culture. As a professional providing $\mathrm{CCC}$, the nurse is able to incorporate elements of care into the residents' care fluidly, often without having to be asked to do so by the residents. A Ukrainian $\mathrm{CCN}$ is able to anticipate the needs of the Ukrainian residents.

Cultural insight, as a concept, is not well understood in the literature as evidenced by the lack of research or scholarly content around it. From the narrative findings of this study, a conclusion is reached that cultural insight is not text-based knowledge. Rather, it has an ineffable quality to it, as it is such an extreme and, almost intimate, understanding 
of the expanses of the culture that it cannot be described in words. When providing CCC to residents who are of the background common to the nurse, cultural insight is the guiding principle of care, as becomes apparent through the participants' narratives of personal experiences of relying on their personal knowledge of the Ukrainian culture in providing CCC to older Ukrainian immigrants.

Greater than an ethnic match. As was mentioned earlier, matching up clients and residents based on their cultural, ethnic or religious backgrounds has been considered in the literature as a potential approach for enhancing the delivery of CCC (Emami et al., 2000; Gilchrist \& Rector, 2007). Such a culturally-equivalent dyad is supposed to remove communication barriers between the care provider and care recipient (Heikkila et al., 2007), promote trust within the therapeutic relationship (Benkert et al., 2006) and enable individualized care (Heikkila et al., 2007; Stuart \& Nielsen, 2011). Being of similar cultural background, however, does not make the client-provider interaction immune to misunderstandings.

Some scholars argue that knowing the cultural background itself does not translate into CCC; the care provider should also have general skills associated with cultural competence in order to be able to use that personal understanding of the culture effectively in her care (Schim et al., 2007). Others, such as McGinnis et al. (2010) and Waxler-Morrison et al. (2005) also emphasize the persistence of inter-generational and intra-cultural variations, as the result of differences in age, social status, and personal experiences; collectively, these factors may create instances of misunderstandings between individuals of the same cultural backgrounds. These differences, however, have not been acknowledged by the participants of this study as barriers to CCC. Instead, both 
Nadiya and Vera acknowledge the potential for the difference to exist, but they also reflect on their willingness to accept the viewpoints of residents that differ from their own in a respectful manner, and then subsequently to incorporate the clients' preferred ways into their own care. For example, Nadiya has a habit of bringing homemade Ukrainian food for the residents even though she expects that her product may come under scrutiny from the residents. Since she is aware that in Ukrainian culture it is acceptable for the older adults to teach the younger generation the ways of life Nadiya is able to appraise the criticism with respect to this understood cultural tradition.

Cultural insight as distinct from cultural intuition. Intuition is a close linguistic relative of insight; in fact, the two are synonyms, yet I question whether cultural insight can be substituted for cultural intuition. Intuition as an element of clinical competence and a nursing skill was introduced in the literature by Benner and Tanner in 1987. Their collective writing was an extension of Benner's earlier work on the process of transitioning between a novice and an expert nurse. The colleagues acknowledge that an expert nurse uses intuition when making clinical judgments that she has developed through her extensive background of professional experience (Benner \& Tanner, 1987). The validity of the nurse's intuition has come under considerable scrutiny in the age of evidence-based practice, since there is lack of empirical data demonstrating the exact process of intuition development and how it functions (Nyatanga \& deVocht, 2008; Woolley \& Kostopoulou, 2013). Despite this, there is research that has proven the effectiveness of nurses' intuition in clinical care (Pretz \& Folse, 2011). Yet the examples that come through Nadiya and Vera's narratives create an image of a phenomenon that is different from cultural intuition. Perhaps the distinction lies in the extent of immersion in 
the culture. With cultural instinct, especially when considered from the point of view of Benner and Tanner (1987), the understanding of the culture develops from the interaction with the clients of diverse cultural backgrounds in practice. Cultural insight that predominates in Nadiya's and Vera's CCC comes from the personal experiences of having grown up in the culture. The participants' immersion in the culture and experience with it is more dynamic, varied and extensive. They do not learn from others, but instead they learn for themselves by being part of the culture. However, even when considering this interpretation, the difference between the two concepts is very ambiguous.

A recent article with a focus on cultural intuition was located in the Journal of Obstetric, Gynecologic \& Neonatal Nursing by Henderson, Reis and Nicholas (2015). The researchers were concerned with CCC for clients and their families in the neonatal intensive care unit, an age group at the opposite end of the life spectrum from the age group of older adults whose care is considered in this study. Henderson et al.'s research, however, was conducted in Canada, and gives some additional context to the understanding of cultural intuition from the perspective of HCPs. A total of $58 \mathrm{HCPs}$ had participated in this qualitative study that focused on understanding the perceptions and experiences of HCPs of providing care to new immigrant families. Among the uncovered themes was lack of intuitive perceptions of the family's needs. The HCPs acknowledged that part of being able to provide $\mathrm{CCC}$ is having the ability to perceive the diverse cultural norms of the clients. When caring for families of an unfamiliar cultural background, the HCPs in Hederson et al.'s study encountered difficulties in anticipating the clients' needs. This conclusion is in contrast to the narrative findings of the current study, where Nadiya and Vera acknowledge that they are able to anticipate the residents' 
cultural needs without excessive effort. This lack of effort does suggest an intuitive element that Nadiya refers to as you "just know". Furthermore, Nadiya and Vera are able to reflect upon the cultural traditions of the group and understand the client's current behavior in this context. Nadiya's narrative about understanding the reasons behind Mr. M's refusal to take his medication is an example of this dimension of cultural insight.

It could, however, be argued that Vera's experience of cleaning the crumbs off Mrs. H's bed demonstrates the gaps within cultural insight. In acting out of habit, it seems Vera had failed to consider the cultural needs of residents at that moment. This incident, though, ended up being a professional learning experience for Vera, who now feels competent in providing CCC and guides the younger nurses. The participants in Henderson et al.'s (2015) study acknowledge that one of the approaches they adopt to expand their cultural intuition and to deepen their understanding of the cultural norms of their clients is paying attention to their interactions. These findings are in line with Nadiya and Vera's explanations that willingness to learn and being attentive are important to the CCN's ability to provide CCC. However, it appears that, according to Henderson et al., simply observing and being attentive to culture can lead to enhanced intuitive understanding of it, yet Vera and Nadiya's narratives describe cultural insight as something that emerges from within; it is not a learned, but a lived quality.

An alternative interpretation of Vera's experience with Mrs. H can also be examined with respect to the principles of cultural match that is recognized in healthcare as a potential approach for enhancing the quality of CCC. The dry bread held meaning to the resident, as it was representative of Mrs. H's experience of surviving the Holodomor. Vera, however, comes from a younger generation of Ukrainians, born after the time of 
the national famine. Although both Mrs. H and Vera self-identify as being Ukrainian, the generational differences between the two women exposed them to different historical experiences and quality of life. This mismatch in the context of their experiences with culture raised the potential for misunderstanding and points out the need for education even when the caregiver is of the same cultural background. Bonder and colleagues (2001) acknowledge that intergenerational gaps between members of the same cultural groups may result in misunderstandings. What seems to be more important in this incident is how Vera's CCC changed as the result of this incident, rather than a verified explanation of the incident itself.

I+Thou. In addition to examining the concept of cultural insight from the practical standpoint, it is possible to consider its potential philosophical underpinnings. It seems that, to some extent, cultural insight is reflective of the work of previous scholars, going at least as far back as the writings of Plato who described in the Phaedo the phenomenon of "being". Plato writes about a recognition that may occur whereby one may encounter an individual who is instantly recognizable and understood even though they have not yet met. In Plato's writing it is evident that this familiarity and recognition is based upon the belief that the soul is immortal, and that the essence of that person's way of "being" is recognizable when the soul is born again in the next life (Cahn, 1999; Heidegger, 1962). This philosophical thinking has persisted through many generations of philosophers who have further explicated the notion that there are qualities or characteristics of the individual that one can appreciate, recognize, or "sense" despite never having encountered the person before. Such intimate knowing of the other comes through the narratives of Nadiya and Vera who equate the residents they care for as being 
like family. The notion of cultural insight, however, does not appear to stem out of the belief of re-incarnation or a pre-existing soul mentioned in Plato's writings. Rather, the origins of cultural insight seem to be reflective of the work of more recent philosophers and scholars such as Heidegger, Buber and Tom Kitwood (Kitwood, 1997), who have both stated that one can profoundly understand another person through application of a moral imagination based on well-developed awareness and knowledge of what it means to be "another" person, distinct, but yet profoundly similar to oneself. The Heideggerian view is that one's understanding of the world is a priori, and this deep understanding of another is grasped as a result of the cultural and linguistic practices that have developed through relating with a particular culture (Leonard, 1999). These works are a nod to the emergent understanding of cultural insight as the ability to understand another's cultural needs based on a shared experience of having grown up in the same culture. However, one is never born already knowing all things about their culture. Being of Ukrainian backgrounds means the individual has been raised in the traditions of that culture; the person was not actually born Ukrainian, but instead learned what it means to be Ukrainian through experience. If the nurse's cultural insight develops through the experience of being immersed in the culture, is it possible then to train other nurses on cultural insight as a means of promoting CCC?

In their narratives, Nadiya and Vera do make reference to nurses who, despite not being of Ukrainian heritage, are also adept at providing CCC to older Ukrainian immigrants. This seems to contradict the earlier described notion of cultural insight as a deep and intimate knowledge, and moves it towards the field of skills that can be learned through relational interactions with the residents. The writings of Martin Buber (1958) 
can help shed some light on this interpretation. From the standpoint of existentialism, Buber, a philosopher, reflects on how people engage with the world. He proposes that people can use their imagination to enter into what he calls the I-Thou relationship. The IThou is concerned with the living relationships, attachment and interactions. The "I" stands for the person, while the "Thou" refers to the other with whom the person engages; it can be an inanimate object, an animal, a spiritual entity or another human. When the I encounters the Thou, Buber explains that the two are changed and transformed through the relationship that forms between them. The hyphen in the I-Thou, as explained by Dr. Lori Schindel Martin (Personal Communication, June 22, 2015), represents the way individuals can intuitively 'know' and 'recognize' each other without actually having met before. In applying this idea to the relationships the study participants describe forming between themselves and the residents, the nurses are able to anticipate the needs of the residents without having been told to do so. The narrative data of the study includes descriptions of how the nurses, having been born in Ukraine, are able to recognize in their residents the essence of what it means to be Ukrainian, even if they come from two different regions of the country with different dialects.

Buber (1958) acknowledges that encounters between I and Thou are commonly fleeting in nature, therefore, the individuals are often left yearning for more. Despite this brevity of encounter, the individuals are forever changed by it, as there is mutual reciprocity that transpires between the two (Buber, 1958). From the participants' narratives, it is also evident that some people who have been born within the culture do not necessarily enter into the I-Thou relationship with the residents, while others, who were born outside of the culture are able to enter the I-Thou relationship from the 
connectivity perspective. Nadiya brings forward an example of a nurse from her practice who was not willing and able to incorporate the perspectives of the clients into the nursing care she delivered, despite being ethnic Ukrainian. Some people, it seems, are too bound by the traditional professional standards of the medical model, where the HCPs care only for the physical needs of their clients. This narrow view of the needs of the resident excludes the focus on their cultural, social or historical backgrounds.

If the hyphen in the I-Thou represents the reciprocity of the relationship between the two people, then, perhaps from the perspective of cultural insight, the hyphen can be replaced by a plus sign to indicate that the two entities function in similar manner due to their shared cultural background. This would reinforce the participants' narratives of the closeness and familiarity they experience towards the residents. I+Thou could, therefore, represent what it means to be a Ukrainian nurse providing CCC to Ukrainian older immigrants in a Ukrainian-specific LTCH. Fuller (2003) challenges the mainstream belief that HCPs must first develop knowledge of the clients' cultures before they are able to provide CCC. This approach may prevent the HCPs from caring for immigrant clients as they begin to harbor feelings of fear and concern of making a mistake (Fuller, 2003). Instead, Fuller explains that the HCPs should focus on reflecting on their interactions with the culturally diverse clients, which will help develop better understanding of themselves, as well as the needs of others. This is how the hyphen becomes a plus - the two learn about each other and about themselves. A nurse who is aware of her own values and beliefs will be able to provide CCC more effectively to others despite the differences stemming from the cultural disparities that may exist between them (Fuller, 2003). 
Why cultural insight is not ethnocentrism. It is important to explicate that the findings of this study are very different from the phenomenon of ethnocentrism, which is commonly viewed as an impediment to CCC (Dudas, 2012). While, both participants described moments within their care of Ukrainian older immigrants where their actions have been guided by their deep knowledge of Ukrainian culture they did not voice any prejudiced remarks towards other cultural groups. The shared cultural background with their clients serves as a point of reference in terms of communication, assessment and treatment. Most importantly, both expressed that, while it would be a challenge for them initially to work in a multicultural LTCH, they would be willingness to learn about the preferences of these culturally diverse clients. Despite the fact that they express pride in their Ukrainian heritage, and their level of cultural proficiency is directly related to their own personal experience as a Ukrainian, they explicitly state that CCC can be delivered by nurses from very different cultural backgrounds. What is essential is the willingness to develop within oneself the skills and attitudes that are associated with cultural competence.

\section{The Researcher's Reflection: More Questions Than Answers}

The narrative approach of the study, as it is customary with this method, has generated a large volume of data, all of it subjective with respect to the participants' experiences and outlooks. From the data analysis and subsequent discussion, a definition of CCC has been composed that answers the research question on how CCC is conceptualized and delivered from the perspective of the participants of the study.

In the context of long-term care, culturally competent care honors the clients' cultural preferences and aids the clients in living their life in the cultural style they find comforting. The individual's livinghood - the essence of their cultural identity and being - is central to the efforts of keeping the golden middle. CCC is provided 
by a nurse who displays attitudes and skills grounded in cultural insight, which facilitates her ability to anticipate the needs of the clients. The cultural environment of the long-term care facility that incorporates elements of the culture, such as traditional artifacts, language and ethnic foods, supports the nurses' ability to provide culturally competent care by increasing access to the cultural elements of great significance to the residents.

This definition, although lengthy, incorporates the four major themes that are woven through the participants' narratives, including honoring the resident, home is where the varenyky are served, the CCN as the doorway to CCC, and cultural insight as the solid foundation. The definition reflects the fact that the participants are of Ukrainian background and provide the care to Ukrainian older immigrants in a Ukrainian-specific LTCHs. It has become evident that the notions of 'who I am' and 'where I come from' are important to $\mathrm{CCC}$; these origins promote the CCN's cultural healing knowledge mentioned by Stuart and Nielsen (2011). The nurse is able to incorporate her professional knowledge with her personal understanding into the care of her clients. Nadiya and Vera allude to the possibility that care that is void of such cultural healing knowledge lacks depth and relevance from the perspective of the clients.

The cultural specificity of this definition does not necessarily mean that it cannot be used in other contexts, since it speaks to some elements common to the literature on CCC. For instance, the importance of the nurse's attitudes and skills as being integral to $\mathrm{CCC}$ is highlighted in the definition; this aligns well with the focus on the attributes of nurses caring for culturally diverse clients in the literature (Dudas, 2012; Majumdar et al., 2004; Phillips et al., 2012). CCNs who have a deeper understanding of culture are less likely to demonstrate bias towards their culturally diverse clients (Van-Ryn \& Burke, 2000). The proposed definition of CCC emergent from the findings of this study is meant to reflect its complex nature and not intended to promote cultural segregation or to 
discredit the ability of nurses to provide CCC to clients of cultural backgrounds that differ from their own. As this is narrative data, it means this understanding is specific to the experiences of the participants of this study. It cannot be said with absolute determination that ethnic match is the only approach for enhancing CCC. What became evident as the result of this study is that there are factors that are important for promoting CCC in a LTC facility that is meant to feel like a cultural home for the residents. Language, cultural artifacts and ethnic foods have been identified among those elements in this study. If nurses can understand the resident's language, however, while not essential, it does help strengthen the capacity for CCC. The findings of this study do not suggest that if the HCP lacks fluency in the language spoken by the resident, it does not seriously limit the nurse from providing CCC to clients of other cultural backgrounds. What does seem an essential condition for CCC is the capacity of the nurse to develop skills inherent to cultural proficiency and is supported in her efforts by the values of the facility where she is employed (i.e. unrestricted access to translators is offered). Therefore, it is not just the individual CCN who is responsible for CCC, but the system overall should co-operate to promote this type of care. These principles, it seems, can then be applied across the healthcare sector, ranging from LTC to acute care.

To advance the transferability of the composed definition further research is needed, in particular with respect to those areas of blurriness that have been made evident in this study, such as cultural insight and livinghood. Additionally, in order to advance the understanding of CCC in mono-ethnic LTCHs, uncovering the residents' perceptions could be key. The areas of importance for further study are identified below. 


\section{Future Research Directions}

The present study has led to the emergence of a working definition of cultural insight, yet the understanding of the concept is blurred due to the overlap with the concept of cultural intuition. Bringing clarity to the distinctions and the overlap between these two concepts through further research could help answer the emerging questions: Can individuals born outside of the culture be taught cultural insight? Perhaps it is not possible to achieve a level of cultural insight even with individuals who are part of the culture if they are missing the desire to learn how their knowledge can be applied? Completing a concept analysis of cultural insight from an interdisciplinary standpoint could help unfold knowledge about this concept for use within the discipline of nursing, as well as other health sciences (Tofthagen \& Fagerstrom, 2010). The paucity of literature on cultural insight indicates that this is not yet a mature concept; as such, based on the recommendations of Morse, Hypcey, Mitcham and Lenz (1996), cultural insight can be examined via qualitative approach to concept analysis, which, similar to the present study, involves collection of narrative data from participants.

The concept of livinghood as a defining element of CCC has emerged through the narrative data of the study. Its definition, as given previously, is subjective to the experiences of the participants. Its fundamental focus on the needs of the individual with respect to the cultural background present livinghood as a viable addition to the literature on PCC and personhood as described by Kitwood (1998). It toys with the idea that there is more to $\mathrm{CCC}$ than just a focus on the person, and introduces into the care setting additional elements such as historical background, accustomed lifestyle and harmony with the care provider based on a shared cultural background. The significance of the historical context of the person's cultural backgrounds has been recognized in other 
health sciences. For instance, Eiser and Ellis (2007) emphasize the importance of including historical information associated with the group, along with cultural information, in order to provide care that fulfills the cultural needs. Although the focus of Eiser and Ellis' study was on the physician-patient interactions, the colleagues write that understanding of the relevance of historical and social occurrences may help form a connection between the care provider and care recipient that is cross-culturally appropriate. They bring up the example of understanding slavery, home remedies, and the Tuskegeee syphilis study when caring for African American clients. Developing a greater understanding of livinghood through research may help amend the PCC practices to include a focus on the historical, cultural and social context of the client into their care.

Cross et al. (1989), as discussed in the literature review found in Chapter 2, believe cultural competence development advances along a continuum; HCPs and organizations should strive to attain the highest level of competence called cultural proficiency. From the narrative data, it can be deduced that a culturally-oriented LTCH creates the setting wherein cultural proficiency can be developed and thus may be practiced by including traditional cultural elements in its environment. It is worth exploring whether the cultural environment of the facility is a springboard for cultural proficiency of the HCPs, as this could provide clue as to how the physical setting of the healthcare facility affects health outcomes.

Since the participants of the study were nurses, the understanding of CCC that came through was positioned from the perspective of the frontline care providers. Due to the time constraints associated with the Master level of studies, it was not feasible to explore the same research question from the perspective of the immigrant older residents 
of the mono-ethnic LTCHs, or their family members. As was made apparent in the study by Hikoyeda and Wallace (2001), residents of mono-ethnic LTCHs and their families have varying expectations in regards to CCC. Furthermore, while language, cultural artifacts and ethnic foods have been identified by Nadiya and Vera as important elements of culture with respect to $\mathrm{CCC}$, there may be other elements of culture that can affect the clients' preferences. Exploring the experiences and perceptions of Ukrainian older immigrants residing in Ukrainian-specific LTCHs in the GTA may highlight their understanding of $\mathrm{CCC}$, and further inform the direction of improvements that may be needed to enhance the quality of life for this group.

Greater understanding of CCC in ethnic-specific LTCHs may be further explored by involving other representatives of the workforce in research, aside from nurses. For instance, the participants of the current study made references in their narratives to staff members who are of diverse cultural backgrounds, reflecting on how they interact with the Ukrainian residents. Studying the experiences of culturally diverse staff with providing CCC, either through narrative or ethnographic studies, may give further insight into how CCC is defined and delivered to Ukrainian residents in Ukrainian-specific LTCHs by non-Ukrainian HCPs.

\section{The Outliers}

Once the data analysis was complete, it became apparent that there are some peripheral themes that have emerged from the collected data. For instances, Nadiya devoted some of her narratives to mulling over the existence of familial guilt in instances of transitioning older family members into long-term care. Together, we explored how this guilt is associated to the emerging theme of lack of time as a barrier to $\mathrm{CC}$ care, yet 
the literature on familial guilt as a concept of LTC placement was left untouched. In reflecting on our conversations further, I have decided that exploring this concept would not add significant value to the study at hand. However, the complexity of this concept presents it as a potential area for future research. Among the potential future research directions related to the phenomenon of familial guilt is understanding if and how it manifests in the relationships between family members and HCPs. Identifying the potential implications of familiar guilt on the quality of CCC provided by the CCNs also stands out as a viable research direction. For instance, the dry bread that Vera had uncovered in Mrs. H's bed was brought in by her family, however, the incident involving the bread was a defining moment in Vera's experience with providing CCC. As the family continues to be involved in the care of the residents, it seems fitting to understand their contributions to CCC.

The participants had also acknowledged that they did not receive any formal training from their employer LTCHs on CCC upon hire. These mentions have been used as supporting information for the major themes that have been discussed as part of the findings of this study. The quantitative researcher in me is curious to understand whether the quality of care of the residents of a mono-ethnic LTCH is affected by the lack of training offered to the staff. Additionally, it would be valuable to explore whether other mono-ethnic LTCHs in the GTA offer such training and how it is structured to attain optimal results. In fact, what are the optimal results with respect to training HCPs on CCC? Based on the findings of this study, in the following section on implications, I put forward suggestions as to the mode of training that seems likely to be effective in promoting CCC at the mono-ethnic LTCHs. 


\section{Implications for the Healthcare Organizations: Immersed Training}

The narratives of Nadiya and Vera illuminate the concept of CCC from the perspective of the CCNs; this was the purpose of the study. Based on the interpretation of these narratives some inferences can be made regarding the implications of the uncovered findings for the mono-culture healthcare organizations. The CCNs function and provide CCC within the context of the organization where they are employed, however, both participants acknowledge the lack of training on CCC available with their employers. Lack of training and knowledge has been named by the public health nurses of Starr and Wallace's (2009) study as a barrier to their ability to provide CCC. Kitwood (1997), in his work on improving the care of people with dementia, recognizes that a tailored orientation, or induction as he labels it, is important for promoting a caring organization. A period of orientation is an opportunity for new staff to gain confidence and familiarity with the residents and the organization (Kitwood, 1997).

Kitwood (1997) wrote that an essential element of orientation for new hires who are to work with people with dementia is content on the illness itself and the effective care strategies. Translating this concept to CCC for older immigrant adults, it becomes evident that training and information related to the culture of residents for whom the CCN will be caring for is a valuable part of the orientation process. Kitwood emphasized that an employee well versed in the needs of the client population should be appointed as a source of reference for the trainees. When training staff on $\mathrm{CCC}$ with respect to a specific culture, it may be appropriate to involve the gatekeepers of that community (i.e. spiritual leaders) who hold the insightful understanding of cultural traditions. A further step to orientation in a mono-culture LTCH could be the opportunity for the new staff to participate in some of the traditions of the culture. For example, making varenyky and 
sharing an ethnic meal with the residents of the Ukrainian LTCH may create the space for the staff to learn about the residents from the residents. Dunn (2005) emphasizes that HCPs should take the time to build rapport with the clients, their families and even their cultural communities in order to enhance their own understanding of cultural variations.

Recognizing the high degree of interdisciplinary collaboration that takes place in LTC, training could be considered for all levels of staffing (i.e. dieticians, physical therapists, nurses, supporting and custodial staff) (Pecukonis, Doyle \& Bliss, 2008). As was mentioned earlier, training on CCC has been associated with improved teamwork (Lange et al.,2013). Similarly Vera identifies collegial support is a valuable to CCC. Basing her work in theories that emphasize the importance of relationships in coordinating work for better outcomes, Gitteell (2011) writes that shared knowledge, shared goals and mutual respect among individuals are the dimensions of relationships that are integral to the process of coordination.

Coordination, according to Gittell, Weinberg, Pfefferle and Bishop (2008), may aid in achieving the principles of individualized approach instituted in LTC settings by facilitating the organization's capacity to address the interdependent tasks associated with care. Gittell et al. (2008) investigated the impact of relational coordination on quality outcomes and job satisfaction across a sample of nursing aides and residents in 15 nursing homes in US. According to their findings, both residents' quality of life and the staff's job satisfaction improve in tandem with enhanced coordination among nursing home staff. Training the staff on the principles of cultural competence, the attributes of the residents' cultures and the approaches for promoting relational cooperation may be of significant value in LTCHs that have adopted the PCC philosophy, promoting the 
integration of the residents' cultural backgrounds into the care routine. Principles of coordination are also of benefit in instances when staff function under time constraints (Gittell et al., 2008). Lack of time as the barrier to CCC was identified by Nadiya. Through immersed training on CCC and relational coordination, staff of LTCHs may learn to collectively function to overcome challenges.

Although the type of immersed orientation proposed here is a novel concept at the system's level, an enhanced degree of cultural competence has been noted among nursing students who participate in cultural immersion training (Larsen \& Reif, 2011). A variation of this orientation approach is currently available at Baycrest Health Sciences facility in Toronto, which offers in-depth training for staff on caring for the aging Holocaust survivors (Baycrest Health Sciences, 2015). Among the features of the program is a practice manual containing information on the Holocaust and expert advice on caring for the survivors, as well as an educational video on managing difficult behaviors in persons with dementia (Baycrest Health Sciences, 2015). Helping the staff familiarize themselves with this culturally-significant historical event fosters a greater degree of appreciation and understanding of the clients' needs and background. Richer CCC may be possible through such integrative training.

In order to facilitate the availability of training tailored to the needs of the residents, it is essential to supply funding for such initiatives. As mentioned earlier, the LTCH Act (2010) does not address the issues around funding of CCC training. I would, therefore, advise the Ministry of Health and Long-Term Care advisors to consider restructuring the budget in a manner that mandates a specific portion of the money made available by the province to be applied towards training around CCC. Based on the 
current study findings, providing such training for internationally-educated professionals may alleviate some of their concerns around caring for culturally-diverse clients.

\section{Policy Implications: Hiring Practices}

Barrass (2012) argues that increasing the availability of culture-specific LTCHs may help alleviate some of the healthcare challenges associated with aging for the culturally diverse adults. Gelfand (2003) confirms, writing that having culturally familiar practices may reduce stressors and barriers to accessing quality care. For example, when making the transition into $\mathrm{LTCH}$, individuals rely on other members of their ethnocultural group for support (Chau \& Lei, 2011). Ethno-specific LTCHs are not highly prevalent in Canada; this is primarily attributed to the common culturally-mediated opposition to institutionalization of older adults among many ethnic groups (Barrass, 2012; Brotman, 2003). Although the trend has been shifting in more recent years; Barrass explains that institutional care has become more acceptable among the immigrant families of some European groups, such as Italians, where the grown children are more likely to follow Western norms. In later generations, therefore, the importance of some of the cultural elements may vary. However, if we are to accept the notion that a degree of cultural norms persist through generations, the descendants of the first wave of Ukrainian immigrants to Canada, who are now entering LTCHs, may continue to hold an understanding of Ukrainian culture and expect that they will receive CCC. Furthermore, there will be subsequent waves of immigration, whose parents will be brought to Canada with a modern outlook on Ukraine, but still expect some kind of culturally relevant and sensitively delivered care by someone who speaks their language and understands their background. 
It seems appropriate to encourage healthcare organizations to develop hiring policies that, while they would expect the potential employee to follow the Canadian rules and regulations, they would simultaneously give preference to individuals who have the capacity to connect to the residents in culturally-meaningful manner, like Nadiya and Vera do with the residents at their Ukrainian specific LTCHS. I hold that LTC facilities should have the power to institute such policies, recognizing how significant it is for the residents to be the recipients of CCC. Of course such hiring practices would need to be within the law, so that they are not discriminatory towards any one particular segment of the HCP population. In order to sustain the cultural sensitivity as a whole, the organizations would benefit from instituting policies that, for instance, have a certain percentage of hired staff who are of the specific cultural background and are fluent in the language. I do recommend further investigation in this area, in particular with respect to how to sustain a culturally-specific LTCH in a way that would not put labor laws and fairness to employees at risk.

\section{Strengths and Limitations}

The narrative methodology built into the study a number of approaches to promoting rigor.

\section{Strengths of the Study}

From the perspective of the researcher, a major strength of this study was its actual methodology of narrative research. Approaching the phenomenon of interest, in particular the conceptualization and delivery of $\mathrm{CCC}$, from a qualitative perspective helped collect a large volume of descriptive data based on the participants' personal experience with the concept. As narrative research, this was the first study to give 'voice' 
to the Ukrainian nurses working in a Ukrainian-specific LTCH. Gathering the data from the perspective of the participants gave valuable insight into $\mathrm{CCC}$, and set the direction for further research.

Following the narrative methodology of Lieblich and colleagues (1998) also presented the opportunity to develop the data analysis process in response to the data collection period. As a result, I was able to consider visual data in the form of photographs as a way of building on the verbal data collected through interviews with the participants. I was, therefore, able to confirm the interpretations of the data through this additional layer of insight into the concept being studied. Since Lieblich et al. acknowledge the fluidity of the data analysis process, it was possible to make alterations to this step of the study in response to the data that was emerging.

\section{Limitations of the Study}

This study has several limitations with respect to the transferability of the findings and the use of disposable cameras for visual data collection.

It works for us, but not for all. The findings of this study, due to the small sample size of two participants, cannot necessarily be generalized to the nursing population in general. Although it is not the aim of narrative research to produce findings that can be replicated, the data collected herein is specific to the Ukrainian diaspora in the GTA. Additionally, there is homogeneity of the participants with respect to ethnicity. While some of the interpreted findings may be reflective of other immigrant communities, in particular those elderly immigrants originating from Eastern Europe, further research would be advisable with respect to implementing the training and education approaches proposed. Perhaps greater interest could have been generated if the 
recruitment period was longer, or if I had advertised through more churches. At the time, however, I had felt that I had approached all of the most influential parishes within the Ukrainian community.

As an interprofessional setting, the Ukrainian LTCH brings together representatives of various disciplines whose understanding of CCC may differ from that of nurses. As the participant sample was limited to the nursing staff of these facilities, the gathered data does not necessarily account for the diversity of perspectives of HCPs around CCC. Furthermore, non-Ukrainian individuals are part of the workforce at the Ukrainian LTCHs; while it was not the intent of this study to compare their experiences of CCC with those of Ukrainian staff, considering their narratives may further advance the understanding of the concept.

We should have taken 'selfies' first. Among the limitations of the data collection process was the use of disposable cameras as the tools for collecting the visual data. The features of the disposable cameras differ from the digital units that are commonly used today; there is no option for the photographer to review the photograph that has been taken. On the one hand, this is an advantage, since the captured image given to the researcher cannot be altered by the photographer in advance. This may preserve the meaning of the photograph. On the other hand, however, this can be a disadvantage since the photographer cannot make certain that the image has, in fact, been caught on film. Furthermore, the quality of the photographs in terms of color saturation, sharpness and exposure can be on the lower side.

The limitations of the disposable camera were pronounced in the experience of Nadiya. Some of the photographs she remembers taking were not actually captured on 
film, while the other photographs came out darker, with the objects cut off around the borders of the shot. As was explained to me by the processing lab, the photographs were taken from too close of a distance, which significantly reduced the amount of lighting. As the equipment used for the activity was a disposable camera, there are certain limitations that are inherent to this device. For optimal results, photographs are to be taken at least four feet away from the object and in broad daylight. Nadiya had attempted to capture images indoors from up close. In future studies, it would be advisable to provide the participants with more detailed directions for using the equipment, along with the opportunity for them to practice using the equipment in the researcher's presence. Additionally, alternative visual data collection tools may be considered, such as use of video recording equipment.

\section{Summary}

My experience affirms for me that Stuart and Nielsen's (2011) innovative article about how quality of care and relationships are enhanced because of the shared cultural background has some practical value. As I wrote in Chapter 1, I find there to be a feeling of ease and comfort in my interactions with Ukrainian clients in my own nursing practice. I often feel that I am able to provide a high quality of care to my Ukrainian peers, as I am able to understand their language and their needs. The findings of this study have led to evidence that supports this notion of a shared cultural background as a potential element of meaningful and respectful CCC.

Similarly, being of Ukrainian background was important to my role as a researcher. I was able to connect with the participants on a deeper lever by acknowledging that I recognize the meanings of their experiences of providing CCC to 
older Ukrainian immigrants. Whenever they would make a reference to Ukrainian traditions or beliefs, I held an inherent understanding of what those properties mean to Ukrainians. There was something about the fact that I am Ukrainian that was of significance to Nadiya and Vera. For instance, since I knew the social conventions, such as use of "Pani" as a way to address the participants, I could establish trust that other researchers might not have been able to do. I am of the belief that this cultural equivalency between the participants and myself had, perhaps, allowed for deeper understanding. Although both the participants and I belong to the fourth wave of immigrants, there is an inter-generational gap between us. Still, given that the participants had lived longer in Ukraine than I had, we each hold an understanding of the core principles of Ukrainian culture. It is this cultural insight that reinforced mutual respect and understanding between us.

The findings of this study have helped to further unveil the perceptions and experiences of frontline nurses with providing $\mathrm{CCC}$ to older immigrant residents of the culture-specific LTCHs. Although the attained understanding is specific to the Ukrainian immigrant community in the GTA, the four major themes emerging from the data during the third level of analysis speak to the general principles of CCC.

Recognizing the important role nurses and the healthcare setting hold for CCC, attention should be given to these areas in terms of further development. Additionally, these findings reinforce that incorporating the needs of the client, in particular cultural preferences, into their care is an essential element of CCC. Finally, the two novel phenomena that have emerged from the data, the concept of livinghood and cultural insight, have laid the foundation for further scholarly research. The data collected was 
truly a unique look into the process of conceptualization and delivery of CCC by Ukrainian nurses to Ukrainian older immigrants residing in Ukrainian-specific LTCHs in the GTA. 


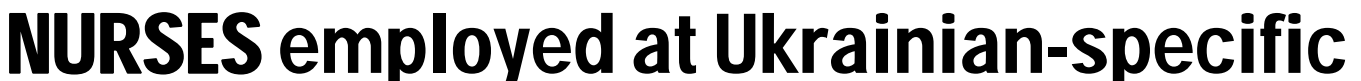

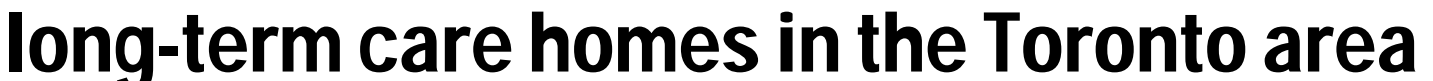

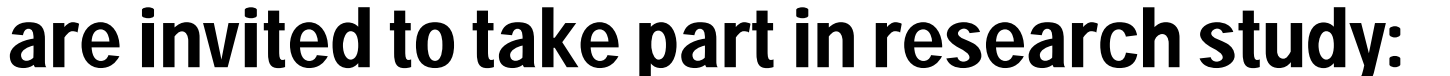

A narrative study of the experiences of providing culturally competent care by frontline staff caring for older Ukrainian immigrants in a monoculture long term care facility

If you volunteer, you will be asked to:

- Attend 3 face-to-face interviews at your convenience

- Complete a short demographic questionnaire

- Take photographs of images from

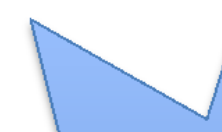

Participants will receive a $\$ 10$ Tim Horton's gift certificate in appreciation of their time. objects using a provided disposable camera

Study participation is voluntary and will involve between 3 to 4 hours to complete study activities. Participants are not expected to incur any cost of participating in the study. Privacy and confidentiality of participants will be strictly upheld.

For more information about this study, or to volunteer, please contact:

Principal Investigator:

Maryana Zaplatsinska, RN

MN Student,

Ryerson University

416-979-5000, ext. 2564 mzaplats@ryerson.ca
Daphne Cockwell SCHOOL OF NURSING

RYERSON UNIVERSITY 


\section{APPENDIX B}

\section{Participant Recruitment Announcement Details}

A narrative study of the experiences of providing culturally competent care by frontline staff caring for older Ukrainian immigrants in a mono-culture long-term care facility

This announcement may be of interest to members of the congregation who are employed as nurses in any of the Ukrainian long-term care homes in the Toronto area.

A student at Ryerson University in Toronto is conducting a research study as part of the requirements for the completion of her Master's degree in nursing. The study is intended to increase the understanding of everyday experiences of nurses who provide culturally competent care to older Ukrainian immigrants who reside in Ukrainian-specific long-term care homes.

Participation in the study is voluntary and will include taking part in three face-toface interviews with the researcher about 2-3 weeks apart. The first and the last interview will last between 60-90 minutes; the middle interview will last 20-30 minutes. The participants will also be asked to complete a brief demographic questionnaire. Additionally, participants will have an opportunity to get creative, as they will be asked to take photographs with a disposable camera provided to them of metaphorical images that reflect their experiences of caring for older Ukrainian immigrants.

The study is recruiting nurses (RNs or RPNs) who have been employed with a Ukrainian-specific long-term care home in the Toronto area for a minimum of two years, are of Ukrainian-background, feel comfortable being interviewed in English, and have at least a rudimentary capacity to communicate in Ukrainian.

Please note that this study is in no way endorsed by the clergy or is associated with our church, and any participation with the study is completely voluntary and will not be disclosed to those associated with the church. If you are interested in learning more about this study, or would like to volunteer to participate in the study, please contact the primary researcher, whose contact information is available on the announcement board (or in the bulletin). 
Shortened Version of the announcement

A student of Ukrainian background is conducting a study as part of her Master's degree with the aim of improving the quality of care provided to the seniors living in the Ukrainian long-term care homes around the Greater Toronto Area. To this extent, she is recruiting nurses, both RNs or RPNs, who have been employed in a Ukrainian specific long-term care home for at least the past two years. All interested parties are encouraged to call 416-979-5000 extension 2564. For further information, please see the advertisement poster on the announcement board.

Ukrainian version of the announcement

Студентка українського походження з Ryerson University проводить наукову роботу 3 метою покращення догляду для сеньйорів що проживають в Українських nursing homes. Для завершення цього проекту потрібна добровільна участь медсестер, RN та RPN, які працюють в українських nursing homes на протязі останніх двох років. Зацікавлених осіб просимо зголошуватися за телефоном 416-979-5000, extension 2564. Щоб більше ознайомитися 3 цим проектом, прошу оглянути плакат на дошці оголошень. 


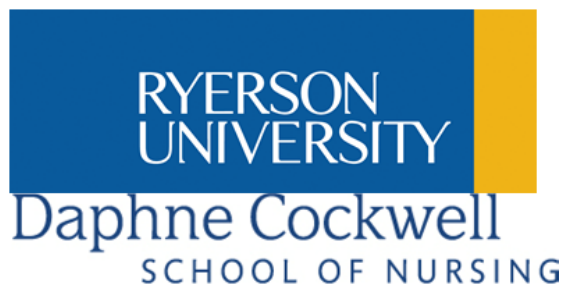

Honorable Reverend NAME,

My name is Maryana Zaplatsinska. I am a Master's level student at Ryerson University working towards completing my degree in nursing. As part of the program requirements, I will be conducting a study of my own design, which intends to understand the perspectives of nurses who provide culturally competent care to older Ukrainian immigrants living in Ukrainian-specific long-term care homes in the Toronto area. I would appreciate your assistance in helping to recruit the participants for this study, which has been approved by the Research Ethnic at Ryerson University.

The study will involve interviewing between 2-4 frontline healthcare professionals who care for older individuals of Ukrainian background in a Ukrainian-specific nursing home. The results of the study will assist in the development of the understanding of the delivery of culturally competent care to older immigrant populations of Ukrainian background. The availability of culturally competent care has been noted in the literature to improve healthcare outcomes for groups of older immigrant who have restricted access to healthcare services due to culturallymitigated behaviors and language disparity.

Included along with this letter are the announcement details, as well as a copy of the recruitment poster with my contact information. It would be of great assistance if the poster could be displayed on the announcement board at the church hall for the duration of participant recruitment (February 28, 2015 to April 6, 2015).

Should you encounter any inquiries about the study from interested participants, please direct these individuals to contact me directly using the contact information below. If you personally have any further questions about the study recruitment process, please feel free to contact me as well.

Principal Investigator:

Maryana Zaplatsinska, RN

MN Student, Ryerson University

416-979-5000, ext. 2564

mzaplats@ryerson.ca
Thesis Supervisor:

Dr. Lori Schindel Martin, RN, PhD

Associate Professor,

Ryerson University

416-979-5000, ext. 4257

lori.schindelmartin@ryerson.ca

Thank you for your time and assistance,

Maryana Zaplatsinska, RN, MN Student 


\title{
APPENDIX D
}

\section{$\underline{\text { Interview Guide - First Interview }}$}

\section{A narrative study of the experiences of providing culturally competent care by frontline staff caring for older Ukrainian immigrants in a mono-culture long-term care facility}

\author{
Dear Participant,
}

My name is Maryana Zaplatsinska. I am a Master of Nursing student at Ryerson University. I am doing this study as part of the requirement to complete my program with the university. The inspiration for this study came out of my own experience of providing care to culturally diverse older people through my role as a nurse.

Examining the literature, I learned about the practice of ethnic-match, whereby healthcare clients receive care from care providers who share their ethno-cultural background. Such ethnic match is intended to improve the communication between the immigrant care receivers and care providers, and result in improved healthcare outcomes, particularly among the older immigrant group. Taking into account my ethnic background as a Ukrainian immigrant, I became interested in learning more about the experiences of other nurses in providing care to older Ukrainian immigrants in a mono-culture long-term care home. My interest was further spurred by the paucity of literature on the subcultures within the Caucasian ethnic group, as research on culturally competent care is primarily concerned with ethnic minority groups. As a result, I have developed a study that will use narrative qualitative approach to answer the following research question: How do nurses employed in Ukrainian-specific longterm care homes conceptualize and deliver culturally competent care to older immigrant adults? It is anticipated that the results of the study will help better understand the experiences of nurses of providing culturally-competent care to older immigrant adults while working in a mono-culture long-term care homes; As such, the results may be used to direct development of training and education programs for nurses to better address the needs of their culturally-diverse older immigrant client population.

\section{Open-ended questions:}

1. How do you define culturally competent care?

- What comes to mind when you think of Ukrainian-specific culturally competent care?

2. What are some assumptions that you hold about what is culturally competent care? How did you come to hold those assumptions?

3. How do you apply cultural competence in your every day practice?

4. Tell me a little bit about how culture is embodied in the long-term care home where you are employed (i.e. at meal time, in the home décor)?

5. Do you feel that you are adequately prepared to deliver culturally competent care?

6. Have you received any formalized training on culturally competent care? If so, please elaborate/describe.

7. Have you encountered any barriers while providing culturally competent care? 
8. Could you tell me about a particular incident/event where you had provided culturally competent care to an older Ukrainian immigrant resident of a Ukrainian-specific long-term care home?

- Who was involved in this event?

- How do you know that the care provided was culturally competent?

- Was there anything else that you could have done to make the care more individualized to the client's culture?

- What kinds of supports did you receive from colleagues/administration/client's family?

- How did this incident/event make you feel?

9. Could you tell me about some experiences of providing culturally competent care that had led to a negative outcome?

- Who was involved in the event?

- Why was this a negative experience? For whom was it negative?

- How did it make you feel?

\section{Probing questions:}

1. Could you please expand/explain/tell me more about...

2. This is what I think I heard...did I understand you correctly?

3. So what you're saying is "..."

4. What are some of your reasons for thinking/feeling/discussing ...

\section{Closing:}

Is there anything else that you would like to add?

Do you have any questions for me at this time?

Would you like to discuss the next steps of the study? If yes:

I will now explain the photography phase of the study.

REFER TO 2.0 Photography Activity Handout. 


\section{APPENDIX E}

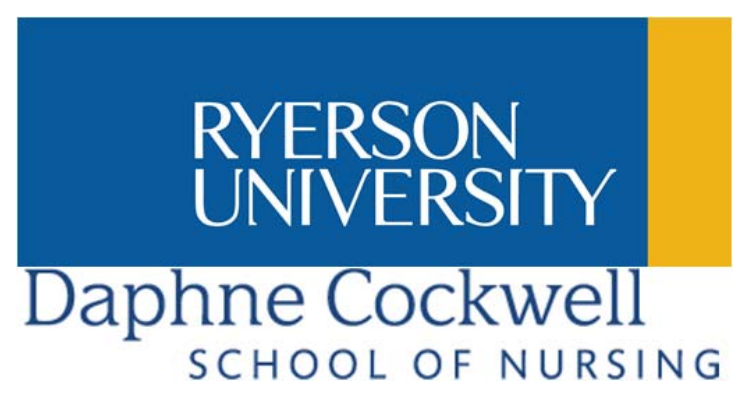

\section{Information and Consent Form}

\section{A narrative study of the experiences of providing culturally competent care by frontline staff caring for older Ukrainian immigrants in a mono-culture long-term care facility}

You are invited to participate in a research study that is being conducted as part of the requirements for the completion of a Master's degree in nursing at Ryerson University, Daphne Cockwell School of Nursing.

Before you give consent to become a participant, it is important that you read the following information provided. Please ask any questions necessary to help you understand what you will be asked to do.

\section{Investigators:}

Principal Investigator: Maryana Zaplatsinska, RN, MN Student, Ryerson University, Daphne Cockwell School of Nursing, Faculty of Community Services, Ryerson University, Toronto, Ontario

Thesis Supervisor: Dr. Lori Schindel Martin, RN, PhD, Thesis Supervisor, Ryerson University, Daphne Cockwell School of Nursing, Faculty of Community Services, Ryerson University, Toronto, Ontario

\section{Purpose of the Study:}

This study aims to understand the perspectives and the everyday experiences of nurses who provide culturally competent care to older Ukrainian immigrants living in a Ukrainian-specific long-term care home.

\section{Description of the Study:}

Your participation in the study will involve sharing of your experiences as a nurse in a Ukrainian-specific long care home providing care to older immigrant adults. Information you provide will be collected during private, face-to-face interview sessions with the principal investigator at a time and location convenient for you. The interviews will take place between late January 2015 and March 2015. There will be a total of three interviews, two of which, the first and the third interviews, will each be approximately 60-90 minutes in length, while the second interview will be 20-30 minutes long. All three interviews will take place approximately 2 weeks apart. The 3 interviews will take place outside of your workday hours at a private location of your choice. With your permission, 
these interviews will be audio-recorded, and will be transcribed by the principal investigator following the interview.

\section{If you agree to participate in this study, you will be asked to:}

1. Take part in total of three face-to-face interviews, the first and third interviews ranging 60-90 minutes, and the second interview lasting between 20-30 minutes in length, each approximately 2 weeks apart, that take place outside of your scheduled work time.

2. Take 4-6 photographs using a disposable camera (provided) of images that reflect your thoughts and feelings with respect to caring for older immigrants adults that reside in a mono-ethnic long-term care home. These photographs are to be taken outside of your workplace or any other healthcare/hospital facility. The photographs can be images of nature, inanimate objects (i.e. clothing, pottery), animals/pets, foods, etc. Photographs are to exclude people, including you, patients, their families, healthcare staff (including professional colleagues), or any capturing of long-term care home's property. The content of these photographs, and your experience of taking the photographs, will then be discussed during the third interview and used in conjunction with all of your interview data to help depict your experiences in both written language and visual representation.

3. Meet with the principal investigator approximately 1-2 weeks after the initial interview for 20-30 minutes at a negotiated time and location convenient to you. At this time, the disposable camera will be collected by the principal investigator to allow the time for the photographs to be developed prior to the third interview. This second brief interview will include a short discussion of your experiences taking the photographs.

4. Be asked to provide consent to being audio-taped during the three interviews. With your permission, these interviews will be tape-recorded and transcribed following the interview by the principal investigator.

If you agree to participate in all the activities associated with this study, it is anticipated that this will involve approximately three to four hours of your time.

\section{The First Interview:}

Upon your consent, the first interview will take place at a private location that is convenient for you. The interview will be audio-taped. Additionally, I will take notes during the interview to ensure that I document the experiences that you share with me. You will be given the opportunity to share your experiences of providing culturally competent care for older immigrant adults who reside in a Ukrainian-specific long-term care home. The interview itself will last between 60 to 90 minutes; you have the right to stop the interview and/or to withdraw from the study at any time. A short demographic questionnaire will be given to you to complete during this interview. At the end of the first interview I will provide you with a disposable camera, along with verbal and written instructions on this activity. At this time, if you wish to continue to participate in this study, we will schedule a 20-30 minute interview in approximately two weeks time at a 
location and time convenient for you so that you could return the camera to me for processing of film.

\section{The Second Brief Interview:}

Approximately 2 weeks after our first interview, we will meet again for approximately 20-30 minutes. At this time, I will collect the camera and have the photographs printed in time for the second interview. I will ask you some questions about your experience with photographing prior to processing the film. If you agree, I will audiotape this conversation. At the end of this interview, I will ask you if you wish to continue to participate in the study. If you agree, we will arrange a time and location convenient for you for the follow-up third interview in approximately 2 weeks.

\section{The Third Interview:}

The third interview will be 60-90 minutes in length and will take place approximately 2 weeks after the second interview at a location and time convenient to you. During the second interview, you will be given the opportunity to share any further information that you might not have voiced in the first interview. I will also have the chance to ask you clarification questions or to elaborate on your comments during our first two interviews. At this time, we will review the photographs that you have taken with the disposable camera. You will have the opportunity to share your reflections and ideas about the meaning the photographs have for you. With your consent, these photographs will be included in their original form in the final research report. These photographs may also be used for presentation and publication purposes, but your identity will always remain confidential.

\section{Potential Risks of your Participation:}

It is possible that you may encounter psychological or social risks by participating in the study. For example, you may remember some unpleasant memories about your experiences related to provision of culturally competent care to older Ukrainian immigrants at a mono-ethnic long-term care home. At any point of the interview, should you begin to feel uncomfortable, you may discontinue your participation in the study, either temporarily or permanently. You may also skip answering any question that you are uncomfortable with.

\section{Potential Benefits of your Participation:}

It cannot be guaranteed that you participation in the study will yield any direct benefits for you. Through participation, you may develop a greater understanding of your own capacity to provide culturally competent care. Your responses will help better understand the experiences of nurses of providing culturally competent care to older immigrant adults.

Should you express interest in learning further about culturally competent care, the principal investigator will have on hand hard copy of the practice guideline document published by the College of Nurses of Ontario titled Culturally Sensitive Care. 


\section{Confidentiality:}

Any and all information collected in connection with the study will be strictly confidential. No identifying information about you or your workplace will appear in any report or publication that results out of the study. Any data that you provide will be represented under a pseudonym of your choosing in interview data and research reports. Data collected during the interviews will be kept in a locked cabinet in the principal investigator's office. During the interviews, you will not be asked to provide any information that will identify you in text.

During the interviews, all the information that you share will be documented through note taking and audio-recorded to ensure that all of our discussion is captured. I will then transcribe the tape-recordings into text to be analyzed. All of the information that may potential identify you will be removed from the transcripts. The electronic files will be stored on a password protected USB flash drive that will be also kept locked in a filing cabinet in the primary investigator's home office. All of the information collected will be kept safely stored for the duration of three years after the study has been completed. In three year's time, raw data files, both electronic and hard copy (e.g. transcripts, consent forms) will be destroyed in a confidential manner.

All feasible efforts will be taken to ensure that neither your coworkers, the management and administrative team at your place of employment, nor your clients or their families can identify you in relation to any of the research data. All data, including visual and interview information, that will be provided by you will be used to fulfill the purpose of this study, as well as for related presentations and potential publications. Full transcripts of the interviews will be seen only by the primary investigator and the thesis supervisor. The remaining thesis committee members will have access to aggregate data only (data presented in a report form) that will be void of your name or any other identifying information. Some of your comments from the interviews may be used as word-for-word quotations to present the results of the study, but you will not be directly identified as the author of those quotations. The photographs that you provide will be used in their original form for the study and in the final research report, but your identity as the photographer will remain confidential. If you express preference not to have your photographs used in the final research report (or select only a few out of the set), this will be respected.

\section{Incentives to Participate:}

As a token of appreciation for your time and participation, you will receive a $\$ 10$ Tim Horton's gift card at the end of the first interview.

\section{Cost of Participation:}

It is not anticipated that you will incur any personal cost for participating in this research study other than the cost of transportation to the meeting places for the interviews. You will be responsible for these transportation costs. 


\title{
Voluntary Nature of Participation:
}

Your participation in the study is completely voluntary and you may choose to stop participating at any time without penalty. At any point in the study, you may decline answering any particular question and you may withdraw from the study altogether. You can also request that audio-taping be stopped either temporarily or permanently at any time during the interviews. Your decision not to participate will not influence that nature of your relationship with Ryerson University, or the Church or Parish in which you were first recruited from, now or in the future.

\section{Questions about the Study:}

If you have any questions about the research now or later, please direct your inquiries to:

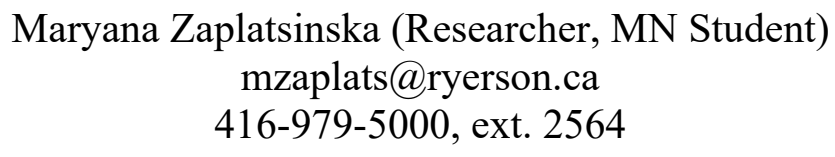

or
Dr. Lori Schindel Martin (Thesis Supervisor) lori.schindelmartin@ryerson.ca 416-979-5000, ext. 4257

The study has been reviewed by the Ryerson University Research Ethics Board. If you have any questions regarding your rights as a human subject and participant in this study, you may contact the research ethics board for information as follows:

\author{
Ryerson University Research Ethics Board \\ Research Ethics Board \\ C/o Office of the Vice President, Research and Innovation \\ Ryerson University \\ 350 Victoria Street \\ Toronto, ON M5B 2K3 \\ T: 416-979-5042 \\ OR via email to rebchair@ryerson.ca
}

\section{Consent:}

By signing below, I confirm that I have read the information in this form, have discussed the purpose of the study and my responsibilities as a participant with the primary investigator. I have had a chance to ask any questions I have about the study. I agree to participate in the study and have been told that I can withdraw my consent to participate in this study at any time. I understand and agree that the photographs I take as part of the visual data collection step of the study will be used for presentation and dissemination purposes, and that my authorship will not be associated with these images. I understand 
that the photos I will be taking as part of this study are of metaphorical images only, and will not depict people (i.e. family members, coworkers, patients). I understand and agree that I will not take photographs in my workplace or anywhere on the property of healthcare institutions. I understand and give my permission for the interviews to be audio-taped and that I have the right to ask for the audio-taping to be stopped, either temporarily or permanently, at any point during the interviews. I have been informed that I am not giving up any of my legal rights by signing this consent agreement. A copy of this consent and information form has been given to me.

Participant Name (please print) , Toronto, Ontario

Participant Signature Date:

Name of Investigator

Signature of Investigator Date 


\section{APPENDIX F}

Photography Activity: Information Handout

Dear Participant,

In our first interview, we had engaged in a conversation about your experiences providing culturally competent care. The information you provided me during that first interview will make up one part of the data used for the study. At this time I am offering you the opportunity to engage in a an artistically creative way of representing your experience of providing culturally competent care to older Ukrainian immigrants residing in a Ukrainian-specific long-term care home in the Greater Toronto Area. I am providing you with a disposable camera that you may use to take photographs of metaphorical objects, such as stills of nature, inanimate objects (i.e. clothing, pottery), pets/animals, or foods meant to be representative of your experiences. This activity will exclude taking photographs of patients, their families, professional colleagues, or the workplace/healthcare settings. Additionally, please refrain from including yourself in any of the taken photographs. These photographs should reflect the feelings, values and ideas of your experiences of providing culturally competent care to older Ukrainian immigrants. However, any photographs that depict people will not be included in the study.

Essentially, the photographs will represent your lived experiences and feelings associated with providing culturally competent care to older Ukrainian immigrants. The 4-6 photographs you take will be used along with the verbal accounts you have shared to enhance and provide further insight into the meaning of your experiences. The photographs will be used in their original form as part of the final research report, and may also be used for publication or presentation purposes. It is ultimately your decision whether I will be permitted to use these photographs for the purposes of the report, publication or presentation. I will respect your decision.

We will schedule a brief meeting in about 2 weeks' time after the initial interview to arrange the pick up of the cameras for processing. I will have two copies of your photographs printed, one of which you will be given as a keepsake of your participation. Together, we will explore the content and the meaning of the photographs during the third interview.

If you have any questions about this photography activity, please do not hesitate to ask me at any time during the study.

Thank you, Maryana Zaplatsinska, RN

Principal Investigator

MN Student, Ryerson University

416-979-5000, ext. 2564

mzaplats@ryerson.ca 


\section{APPENDIX G \\ Second Interview Guide: Camera Collection}

\section{Introduction:}

At our first interview I provided you with a disposable camera to take photographs that reflect your experiences, thoughts and feelings of providing culturally competent care to older Ukrainian immigrant residing in Ukrainian-specific long-term care homes in the Greater Toronto Area. The purpose of our meeting today is so that I can pick up the disposable camera and have the prints developed in time for our follow-up interview, should you decide to continue participating in the study. You will have the option of keeping one set of the prints for yourself.

\section{Questions:}

1. Do you have any questions about the photography activity that you have completed?

2. Do you have any questions, comments or concerns related to any part of this research study thus far?

3. What was your experience of taking the pictures? What did you learn about culturally competent care as a result of reflecting upon and selecting your images?

At this time we are ready to enter the final step of your participation in the study. In approximately 2-3 weeks, I would like to meet with you again for a follow-up interview that will last between 60-90 minutes. During this time, we would both have the opportunity to review the transcripts from our first interview, and to ask clarifying questions. To facilitate this part of the discussion, I could provide you with either a hard copy or an electronic version of the transcript of our first interview ahead of the third and final interview. During our third interview, we will also spend time reflecting upon and discussing the photographs you have taken.

4. Would you like to proceed to a second interview?

If you would like to withdraw from the study at this time, thank you for your participation. If you would like to have a copy of the photographs you have taken, please provide me with an address where I could mail them to you.

If you would like to continue your participation in the study, when is a good date and time for us to meet again? Where would you like to have this meeting? Would you like to read the transcript of our first interview ahead of our final interview? If so, please provide me with an address where I could send the document to you (I could also forward you an electronic copy of this document via e-mail address that you provide). 


\section{APPENDIX H}

Thank you for meeting with me today. Interview Guide: Third Interview

\section{A narrative study of the experiences of providing culturally competent care by frontline staff caring for older Ukrainian immigrants in a mono-culture long-term care facility}

Dear participant,

Thank you for meeting with me for our final interview. During our first interview, I asked you a series of questions about your experiences of providing culturally competent care to older Ukrainian immigrants residing in a Ukrainian-specific long-term care home. Today, I would like both of us to reflect back on our initial discussion and to address any questions/clarifications we might have about the dialogue we shared. We will also take some time to reflect on the photographs that you have taken as a metaphorical representation of your experiences as a nurse caring for older Ukrainian immigrants residing in a Ukrainian-specific long-term care home. I would like to remind you that the consent form you signed indicated that you are free to stop the interview at any point, and have the right not to answer any questions that might make you feel uncomfortable. You may also withdraw from the study at any point without the need to provide an explanation for your decision.

\section{Open-ended questions:}

1. Now that you have had some time to reflect on our initial discussion, are there any other comments that you would like to share with me today that you did not have an opportunity to bring up in our last meeting?

2. Out of the photographs that you have taken, which one, in your opinion, most closely reflects your experience of providing culturally competent care to older immigrants? Please explain.

3. What is the meaning behind this photograph for you?

4. What is the message you are trying to convey through this photograph?

5. How do you think others (i.e. colleagues or residents' families) may interpret these photographs?

6. Let's talk a little bit more about the photography activity itself.

- What were some of your thoughts and feelings as you engaged in this photography activity?

- How did you feel completing this activity?

- Did you encounter any challenges with this activity?

- Did you gain any benefits from this activity?

\section{Probing questions:}

Could you please expand on/explain/tell me more about... In our last meeting you talked about....can you elaborate .... Can you explain what you meant when you said... 


\section{Closing:}

As our time together is coming to a close, is there anything else that you would like to share with me about your experiences of providing culturally competent care to older immigrant adults? Do you have any questions for me?

Thank you for your time and participation. 


\section{RYERSONUNIVERSITY}

\section{RESEARCH ETHICS BOARD}

To: Maryana Zaplatsinska

Nursing

Re: REB 2014-326: A narrative study of the experiences of providing culturally competent care by frontline staff caring for older Ukrainian immigrants in a mono-culture long term care facility

Date: January 21, 2015

Dear Maryana Zaplatsinska,

The review of your protocol REB File REB 2014-326 is now complete. The project has been approved for a one year period. Please note that before proceeding with your project, compliance with other required University approvals/certifications, institutional requirements, or govemmental authorizations may be required.

This approval may be extended after one year upon request. Please be adv ised that if the project is not renewed, approval will expire and no more research involv ing humans may take place. If this is a funded project, access to research funds may also be affected.

Please note that REB approval policies require that you adhere strictly to the protocol as last rev iewed by the REB and that any modifications must be approved by the Board before they can be implemented. Adverse or unexpected ev ents must be reported to the REB as soon as possible with an indication from the Principal Inv estigator as to how, in the view of the Principal Inv estigator, these ev ents affect the continuation of the protocol.

Finally, if research subjects are in the care of a health facility, at a school, or other institution or community organization, it is the responsibility of the Principal Investigator to ensure that the ethical guidelines and approvals of those facilities or institutions are obtained and filed with the REB prior to the initiation of any research.

Please quote your REB file number (REB 2014-326) on future correspondence.

Congratulations and best of luck in conducting your research.

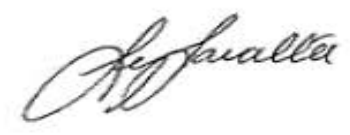

Lynn Lavallée, Ph.D.

Chair, Research Ethics Board 


\section{APPENDIX J}

\section{Demographic Background and Cultural Sensitivity Survey}

A narrative study of the experiences of providing culturally competent care by frontline staff caring for older Ukrainian immigrants in a mono-culture long-term care facility

In order to better understand your experiences as a healthcare professional providing culturally competent care to older Ukrainian immigrants residing in a Ukrainianspecific long-term care home, I would appreciate learning a little more about you. Please provider answers to the following questions to the best of your ability by placing an ' $\mathrm{X}$ ' on the applicable lines. You may skip any question that you do not wish to answer. You may also withdraw from the study at any time.

1. Please specify your gender:

Male _ Female _ Other

2. What is your age range?

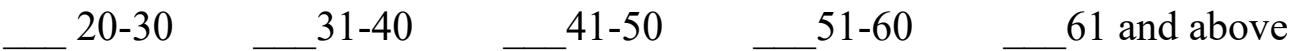

3. Among the following categories, what is your professional designation?

Registered Nurse

Registered Practical Nurse

4. Please indicate your employment status at the long-term care home:

Full-time

Part-time

5. How many years have you been working at the long-term care home where you are currently employed?
2
3-5
6-8
$9-11$
12 and over

6. Have you received any training related to cultural competence while working at a Ukrainian-specific long-term care home?

_ Yes

(If so, please describe:
No 
7. You have:

Been born in Canada

Immigrated to Canada ( $\_$) years ago from

$\left(\frac{}{\text { country }}\right)$

Other (Please specify:

8. How would you describe your cultural background?

9. What language(s) do you speak at home? Please select all that apply.

English

French

Ukrainian

Other(s) Please specify:

Thank you for your time and participation. 


\section{REFERENCES}

Allen, S., Chapman, Y, Karen, F., \& O’Connor, M. (2008). Examining the methods used for a critical ethnographic enquiry. Contemporary Nurse: A Journal for the Australian Nursing Profession, 29(2), 227-237.

Almutairi, A.F., McCarthy, A., \& Gardner, G.E. (2015). Understanding cultural competence in a multicultural nursing workforce: Registered nurses' experience in Saudi Arabia. Journal of Transcultural Nursing, 26(1), 16-23.

Aroian, K. J., Khatutsky, G., Tran, T. V., \& Balsam, A. L. (2001). Health and social services utilization among elderly immigrants from the former Soviet Union. Journal of Nursing Scholarship, 33, 265-271.

Ayres, L., \& Poirier, S. (2003). Rational solutions and unreliable narrators: Content, structure and voice in narrative research. In J. Latimer (Ed.), Advanced qualitative research for nursing (p. 115-134). Oxford: Blackwell Science.

Barrass, S. (2012). An ecological model for culturally sensitive care for older immigrants: Best practices and lessons learned from ethno-specific long-term care. (Master's Thesis). Retrieved from http://digital.library.ryerson.ca/islandora/object/RULA\%3A1551

Barrio, C., Palinkas, L.A., Yamada, A., Fuentes, D., Criado, V., Garcia, P., \& Jeste, D.V. (2008). Unmet needs for mental health services for Latino older adults:

Perspectives from consumers, family members, advocates, and service providers. Community Mental Health Journal, 44, 57-74. doi:10.1007/s10597-007-9112-9 
Beach, M. C., Price, E. G., Gary, T. L., Robinson, K. A., Gozu, A., Palacio, A., et al. (2005). Cultural competence: A systematic review of health care provider educational interventions. Medical Care, 43, 356-373.

Benkert, R., Peters, R. M., Clark, R., \& Keves-Foster, K. (2006). Effects of perceived racism, cultural mistrust and trust in providers on satisfaction with care. Journal of the National Medical Association, 98, 1532-1540.

Benner, P., \& Tanner, C. (1987). Clinical judgment: How expert nurses use intuition. The American Journal of Nursing, 87(1), 23-31.

Berdes, C., \& Eckert, J.M. (2001). Race relations and care- giving relationships: A qualitative examination of perspectives from residents and nurse's aides in three nursing homes. Research on Aging, 23, 109-126.

Bernal, H., \& Froman, R., (1993). Influences on the cultural self-efficacy of community health nurses. Journal of Transcultural Nursing, 4(2), 24-31.

Betancourt, J.R., Green, A.R., Carrillo, E., \& Ananeh-Firepong II, O. (2003). Defining cultural competence: A practical framework for addressing racial/ethnic disparities in health and health care. Public Health Report, 118(4), 293-302.

Betancourt, J.R., Green, A.R., Carrillo, J.E., \& Park, E.R. (2005). Cultural competence and health care disparities: Key perspectives and trends. Health Affairs, 24(2), 499-505.

Briggs, H.E., Briggs, A.C., Miller, K.N., \& Paulson, R.I. (2011). Combatting persistent cultural incompetence in mental health care serving African Americans. Best Practices in Mental Health, 7(2), 1-25. 
Brotman, S. (2003). The limits of multiculturalism in elder care services. Journal of Aging Studies, 17I(2), 209-229.

Browne, A. J., Varcoe, C., Smye, V., Reimer-Kirkham, S., Lynam, M. J., \& Wong, S. (2009). Cultural safety and the challenges of translating critically oriented knowledge in practice. Nursing Philosophy, 10, 167-179.

Boi, S. (2000). Nurses' experience in caring for patients from different cultural backgrounds. NT Research, 5(5), 382-390.

Bonapart, B. (1979).Ego defensiveness, open-closed mindedness, and nurses' attitude toward culturally different patients. Nursing Research, 28(3), 166-172.

Bonder, B., Martin, L., \& Miracle, A. (2001). Achieving cultural competence: The challenge for clients and healthcare workers in a multicultural society. Generations, 25(1), 35-42.

Bourgeault, I.L., Atanackovic, J., Rahis, A., \& Parpia, R. (2010). Relations between immigrant care workers and older persons in home and long-term care. Canadian Journal on Aging, 29(1), 109-118. doi: 10.1017/S0714980809990407

Brennan, A.M.W., \& Cotter, V.T. (2008). Student perceptions of cultural competence content in the curriculum. Journal of Professional Nursing, 24(3), 155-160. doi: 10.1016/j.profnurs.2008.01.003

Brownie, S., \& Nancarrow, S. (2013). Effects of person-centered care on residents and staff in aged-care facilities: A systematic review. Clinical Interventions in Aging, 13(8), 1-10.

Bubar, J. (2014). Handprints of history. Scholastic News, 83I(10), 14. 
Buber, M. (1923). I and Thou (Translated by Ronald Gregor Smith). Edinburgh: T. \& T. CLARK.

Cahn, S.M. [Editor] (1999). Classics of western philosophy. Indianapolis, Indiana: Hackett.

Campbell, D.F. \& Machado, A.A. (2013). Ensuring quality in qualitative inquiry: Using key concepts as guidelines. Motriz, Rio Claro, 19(3), 572-579.

Campinha-Bacote, J. (2002). The process of cultural competence in the delivery of healthcare services: A model of care. Journal of Transcultural Nursing, 13(3), 181184.

Campinha-Bacote, J. (2011). Delivering patient-centered care in the midst of a cultural conflict: The role of cultural competence. The Online Journal of Issues in Nursing, 16(2).

Canadian Healthcare Association, (2009). New directions for facility based long-term care. Retrieved from http://www.healthcarecan.ca/wpcontent/uploads/2012/11/CHA_LTC_9-22-09_eng.pdf

Canadian Nurses Association, (2004). Promoting Culturally Competent Care. Retrieved from http://www.cna-aiic.ca/ /media/cna/page-content/pdffr/ps73_promoting_culturally_competent_care_march_2004_e.pdf

Canadian Nurses Association, (2008). Code of Ethics for Registered Nurses. Retrieved from https://www.cna-aiic.ca/ /media/cna/files/en/codeofethics.pdf 
Canadian Nurses Association, (2010). Position statement: Promoting cultural competence in nursing. Retrieved from https://www.cnaaiic.ca/ /media/cna/page-content/pdf-en/6\%20\%20ps114_cultural_competence_2010_e.pdf?la=en

Capell, J., Veenstra, G., Dean, E. (2007). Cultural competence in healthcare: Critical analysis of the construct, its assessment and implications. Journal of Theory Construction \& Testing, 11(1), 30-37.

Capell, J., Dean, E., \& Veenstra, G. (2008). The relationship between cultural competence and ethnocentrism of health care professionals. Journal of Transcultural Nursing, (19)2, 121- 125.

Carey, R.E. (2011). Cultural competence assessment of Baccalaureate nursing students: Integrative review of the literature. International Journal of Humanities and Social Science, 1(9), 258-266.

Carpenter-Song, E. A., Nordquest Schwallie, M., \& Longhofer, J. (2007). Cultural competence reexamined: Critique and directions for the future. Psychiatric Services, 58, 1362-1365. doi:10.1176/appi.ps.58.10.1362

Caspar, S., O'Rourke, N., \& Gutman, G.M. (2009) The differential influence of culture change models on long-term care staff empowerment and provision of individualized care. Canadian Journal of Aging 28, 165-175.

Chappell, N.L., Reid, C., \& Gish, J. (2006). Staff measures of individualized care for persons with dementia in long- term care facilities. Dementia: The International Journal of Social Research and Practice, 6, 527-547. 
Chau, S., \& Lai, D. (2011). The size of an ethno-cultural community as a social determinant of health for Chinese seniors. Journal of Immigrant Minority Health. 13, 1090-1098.

Clandinin, D.J., \& Connelly, F.M. (2000). Narrative inquiry: Experience and story in qualitative research. San Francisco: Jossey-Bass.

Clandinin, D.J. (2007). Handbook of narrative research: Mapping a methodology. Thousand Oaks CA: Sage.

College of Nurses of Ontario, (2009). Culturally sensitive care. Retrieved from http://www.cno.org/Global/docs/prac/41040_CulturallySens.pdf

Community Care Access Centre. (2015). Choosing a long-term care home. Retrieved from http://mhccac-ltc.com/wait-lists/

Cooper Brathwaite, A. (2005). Evaluation of a cultural competence course. Journal of Transcultural Nursing, 16(4) 361-369.

Cooper Brathwaite, A. (2006). Influence of nurse characteristics on the acquisition of cultural competence. International Journal of Nursing Education Scholarship, 3(1), doi:10.2202/1548-923x.1173

Cooper Brathwaite, A., \& Majumdar, B. (2005). Evaluation of cultural competence educational programme. Issues and Innovations in Nursing Education, 53(4), 470479.

Cortis, J.D. (2004). Meeting the needs of minority ethnic patients. Journal of Advanced Nursing, 48(1), 51-58.

Cortis, J.D. \& Kendrick, K. (2003). Nursing ethics, caring and culture. Nursing Ethics, 10(1), 77-88. 
Cottingham, H., Stoothoff, R, \& Murdoch, D. (1985). The Philosophical Writings of Descartes: Volume 1. New York: Cambridge University Press.

Couton, P. (2014). Ethnocultural community organizations and immigrant integration in Canada. Retrieved from http://irpp.org/research-studies/study-no47/

Crandall, S., George, G., Marion, G., \& Davis, S. (2003). Applying theory to the design of cultural competency training for medical students: A case study. Academic Medicine, 78, 588-594.

Creef, E.T. (2004). Imaging Japanese America: The visual construction of citizenship, nation, and the body. New York: New York University Press.

Creswell, J. W. (2013). Qualitative inquiry and research design: Choosing among five approaches. ( $3^{\text {rd }}$ ed.). Thousand Oaks, CA: Sage.

Coffman, M.J., Shellman, J., \& Bernal, H. (2004). An integrative review of American nurses' perceived self-efficacy. Journal of Nursing Scholarship, 36(2), 180-185.

Cricco-Lizza, R. (2006). Black Non-Hispanic mothers' perceptions about the promotion of infant-feeding methods by nurses and physicians. Journal of Obstetric, Gynecological and Neonatal Nursing, 35(2), 173-180.

Cross, T., Bazron, B., Dennis, K. W., \& Issacs, M. R. (1989). Towards culturally competent systems of care. Washington, DC: Georgetown University Child Development Center.

Diaz-Lazaro, C.M. \& Cohen, B. (2001). Cross-cultural contact in counselling training. Journal of Multicultural Counselling and Development, 29, 41- 56. 
Disman, M., \& Disman, M. (1995). Seniors, Culture and Institutionalization. In R. Neugebauer-Visano (ed), Aging and Inequality: Cultural constructions of differences (pp. 205-225). Toronto: Canadian Scholars Press.

DiCicco-Bloom, B., \& Cohen, D. (2003). Home care nurses: A study of the occurrence of culturally competent care. Journal of Transcultural Nursing, 14(1), 25-21. doi: $10.1177 / 1043659602238347$

Doorenbos, A., Schim, S., Benkert, R., \& Borse, N. (2005). Psychometric evaluation of the clinical competence assessment instrument among healthcare providers. Nursing Research, 54(5), 324-331.

Dudas, K.I. (2012). Cultural competence: An evolutionary concept analysis. Nursing Education Research, 33(5), 317-321.

Durst, D. (2005). Aging Among Immigrants in Canada: Population Drift. Canadian Studies in Population, 32(2), 257-270.

Eggenberger S.K., Grassley, J., Restrepo, E. (2006). Culturally competent nursing care for families: Listening to the voices of Mexican-American women. Online Journal of Issues in Nursing, 11(1).

Eiser, A. R., \& Ellis, G. (2007). Cultural competence and the African American experience with health care: The case for specific content in cross-cultural education. Academic Medicine, 82, 176-183.

El-Amouri S \& O’Neill S (2011) Supporting cross-cultural communication and culturally competent care in the linguistically and culturally diverse hospital settings of UAE. Contemporary Nurse 39, 240-255.

Elliott, B. (2006). Using narrative in social research. London: Sage. 
Emami, A., Torres, S., Lipson, J. G., \& Ekman, S-L., 2000. An ethnographic study of a day centre for Iranian immigrant seniors. Western Journal of Nursing Research, 22, 169-188. doi: $10.1177 / 019394590002200205$

Employment and Social Development Canada. (2015). Canadians in context: Aging population. Retrieved from http://well-being.esdc.gc.ca/misme-iowb/.3ndic.1t.4r@eng.jsp?iid=33

Facione, N. C., \& Facione, P. A. (2007). Perceived prejudice in healthcare and women's health protective behavior. Nursing Research 56, 175-184.

Fahrenwald, N., Boysen, R., Fischer, C., \& Maurer, R. (2001). Developing cultural competence in the baccalaureate nursing student: A population- based project with the Hutterites. Journal of Transcultural Nursing, 12(1) 48-55.

Falicov, C.J. (2005). Emotional transnationalism and family identities. Family Process, 44(4), 399-406.

Fuller, J. (2003). Intercultural health care as reflective negotiated practice. Western Journal of Nursing Research, 25(7), 781-797.

Garneau, A.B., \& Pepin, J. (2015). Cultural competence: A constructivist definition. Journal of Transcultural Nursing, 26(1), 9-15.

Gelfand, D.E. (2003). Aging and ethnicity. New York: Springer.

Giger, J., Davidhizar, R., Purnell, L., Harden, J., Phillips, J., \& Strickland, O. (2007). American Academy of Nursing expert panel report: Developing cultural competence to eliminate health disparities in ethnic minorities and other vulnerable populations. Journal of Transcultural Nursing, 18(2), 95-102. doi:10.1177/1043659606298618. 
Gilchrist, K. L., \& Rector, C. (2007). Can you keep them? Strategies to attract and retain nursing students from diverse populations: Best practices in nursing education. Journal of Transcultural Nursing, 18, 277-285.

Gittell, J.H. (2011). Relational Coordination: Guidelines for Theory, Measurement and Analysis. Retrieved from http://rcrc.brandeis.edu/downloads/Relational_Coordination_Guidelines_8-2511.pdf

Gittell, J.H., Weinberg, D., Pfefferle, S., Bishop, C. (2008). “Impact of relational coordination on job satisfaction and quality of care: A study of nursing homes." Human Resource Management Journal, 18(2): 154- 170.

Giuntoli, G., \& Cattan, M. (2012). The experiences and expectations of care and support among older migrants in the UK. European Journal of Social Work, 15(1), 131147.

Gnaedinger, N. (2003). Changes in long-term care for elderly people with dementia: A report from the front lines in British Columbia, Canada. Journal of Social Work in Long-Term Care, 2(3/4), 355-371.

Gorek, B., Martin, J., White, N., Peters, D., \& Hummel, F. (2002). Culturally competent care for Latino elders in long-term care settings. Geriatric Nursing, 23(5), 272275.

Government of Canada. (2009). Speaking notes for the Honourable Jason Kenney, Minister of Citizenship, Immigration and Multiculturalism, at the Paul Yuzyk Award ceremony. Retrieved from http://www.cic.gc.ca/english/department/media/speeches/2009/2009-06-12.asp 
Government of Canada. (2012). Canadian Multiculturalism: An Inclusive Citizenship.

Retrieved from http://www.cic.gc.ca/english/multiculturalism/citizenship.asp

Government of Canada, (2015). Constitution act, 1982: Canadian Charter of Rights and Freedoms. Retrieved from http://laws-lois.justice.gc.ca/eng/const/page-15.html

Gray, D. P., \& Thomas, D. J. (2006). Critical reflections on culture in nursing. Journal of Cultural Diversity, 13(2), 76-82.

Grove, S., Burns, N., \& Gray, J.R. (2013). The practice of nursing research: Appraisal, synthesis and generation of evidence $\left(7^{\text {th }}\right.$ ed.) St. Louis: Elseview Sanders.

Guba, E. G., \& Lincoln, Y. S. (2005). Paradigmatic controversies, contradictions, and emerging confluences. In N. K. Denzin \& Y. S. Lincoln (Eds.), Handbook of qualitative research (3rd ed., pp. 191-215). Thousand Oaks, CA: Sage.

Gunaratnam, Y., 2008. From competence to vulnerability: Care, ethics, and elders from racialized minorities. Mortality, 13(1), 24-41. doi:10.1080/13576270701782969

Hart, P. L., \& Mareno, N. (2013). Cultural challenges and barriers through the voices of nurses. Journal of Clinical Nursing, 23, 2223-2233.

Hasson, H., \& Arnetz, J.E. (2008). Nursing staff competence, work strain, stress and satisfaction in elderly care: A comparison of home-based care and nursing homes. Journal of Clinical Nursing, 17, 468-481.

Healy, J., \& McKee, M. (2004). Accessing health care: Responding to diversity. Oxford: Oxford University Press.

Hearnden, M. (2008). Coping with differences in culture and communication in health care. Nursing Standard, 23(11), 49-57. 
Heidegger, M. (1962). Being and time. Translated by John Macquarrie and Edward Robinson. New York: Harper \& Row. Reprinted as "Exposition of the Question of the Meaning of Being" Polifroni, E.C. \& Welch, M. [Editors] (1999). Perspectives on Philosophy of Science in Nursing: An Historical and Contemporary Anthology, (pps. 273-302).

Heikkilä, K.\& Ekman, S-L., 2003. Elderly care for ethnic minorities--wishes and expectations among elderly Finns in Sweden. $\square$ Ethnicity \& Health, 8(2), 135-146. Heikkila, K., Sarvimaki, A., \& Ekman, S-L. (2007). Culturally congruent care for older people: Finnish care in Sweden. Scandinavian Journal of Caring Sciences, 21, $354-361$.

Henderson, L., Reis, M.D., \& Nicholas, D.B. (2015). Health care providers' perspectives of providing culturally competent care in the NICU. Journal of Obstetric, Gynecologic, \& Neonatal Nursing, 44, 17-27.

Hikoyeda, N., \& Wallace, S.P. (2001). Do ethnic-specific long-term care facilities improve residents' quality of life? Findings form the Japanese American community. Journal of Gerontological Social Work, 36, 83-106.

Holloway, I., \& Freshwater, D. (2007). Narrative researcher in nursing. Blackwell Publishing: Oxford, UK.

Horne, G. (2011). Canada's policy framework for the utilization of internationally educated nurses. Queen’s Policy Review, 2(2), 37-53.

Huang, T.T., \& Acton, G.J. (2004). Effectiveness of home visit falls' prevention strategy for Taiwanese community-dwelling elders: Randomized trial. Public Health Nursing, 21, 247-256. 
Iecovich, E. (2000). Sources of stress and conflicts between elderly patients, their family members and personnel in care settings. Journal of Gerontological Social Work, 34(2), 73-88.

International Council of Nurses, (2006). Nursing Care of the Older Person. Geneva. Retrieved from http://www.icn.ch/psolder.html

Internet Encyclopedia of Ukraine, (2001). Kobzar. Retrieved from http://www.encyclopediaofukraine.com/display.asp?linkpath=pages $\% 5 \mathrm{CK} \% 5 \mathrm{CO}$ \%5CKobzarIT.htm

Jaffe-Mero, I. (2011). 'Is that what I said?' Interview transcript approval by participants: An aspect of ethics in qualitative research. International Journal of Qualitative Methods, 10(3), 231-247.

Jahng, K.H., Martin, L.R., Golin, C.E., \& DiMatteo, M.R. (2004) Preferences for medical collaboration: Patient- physician congruence and patient outcomes. Patient Education and Counseling, 57:308-314. doi: 10.1016/j.pec.2004.08.006.

Jenks, A. C. (2011). From "lists of traits" to "open-mindedness": Emerging issues in cultural competence education. Culture, Medicine, and Psychiatry, 35, 209-235.

Jones, M.E., Cason, C.L., \& Bond, M.L. (2004). Cultural attitudes, knowledge and skills of a health workforce. Journal of Transcultural Nursing, 15(4), 283-290. doi:10.1177/1043659604268966

Josselson, R. (2007). The ethical attitude in narrative research: Principles and practicalities. In Clandinin, D.K. (2007). Handbook of Narrative Inquiry. Thousand Oaks, Ca: Sage Publications. 
Josselson, R., Lieblich, A., \& McAdams, D.P. (2002). Up close and personal: The teaching and learning of narrative research. Washington, DC:x American Psychological Association.

Kaplan, S., Calman, N., Golub, M., Davis, J., Ruddock, C., \& Billings, J. (2006). Racial and ethnic disparities in health: A view from the South Bronx. Journal of Health Care for the Poor and Underserved, 17, 116-127.

Keats, P.A. (2009). Multiple text analysis in narrative research: Visual, written and spoken stories of experience. Qualitative Research, 9, 181-195.

Kennedy, B., Mathis, C., \& Woods, A. (2007). African Americans and their distrust of the health care system: Healthcare for diverse populations. Journal of Cultural Diversity, 14(2), 56-60.

Kitwood, T. (1997). Dementia reconsidered: Person comes first. Buckingham, UK: Open University Press.

Lambert, S.D., \& Loiselle, C.G. (2008). Combining individual interviews and focus groups to enhance data richness. Journal of Advanced Nursing, 62, 228-237.

Lange, J.W., Mager, D.R., \& Andrews, N. (2013). The ELDER expansion project: Building cultural competence among long-term care workers. Applied Nursing Research, 26, 58-62.

Larsen, R., \& Reif, L. (2011). Effectiveness of cultural immersion and cultural classes for enhancing nursing students' transcultural self-efficacy. Journal of Nursing Education, 50(6), 350-354.

Latimer, J. (2003). Advanced qualitative research for nursing. Oxford, UK: Blackwell Science Ltd. 
Leishman, J. (2004). Perspectives of cultural competence in health care. Nursing Standard, 19(11), 33-38.

Leininger, M. (1991). Culture Care Diversity \& Universality: A Theory of Nursing. National League of Nursing, New York, NY.

Leonard, V.W. (1999). A Heideggerian phenomenologic perspective on the concept of the person. Reprinted from Advances in Nursing Science, 1989, 11(4), 40-55. In Polifroni, E.C. \& Welch, M. [Editors] (1999). Perspectives on Philosophy of Science in Nursing: An Historical and Contemporary Anthology, (pps. 315-327).

Lichtman, M.V. (2013). Qualitative Research in Education: A User's Guide. Virginia Tech: SAGE Publications, Inc.

Lieber, E., Chin, D., Nihira, K., \& Mink, I.T. (2001). Holding on and letting go: Identity and acculturation among Chinese immigrants. Cultural Diversity and Ethnic Minority Psychology, 7(2), 247-261.

Lieblich, A. (1996). Some unforeseen outcomes of conducting research with people of one's own culture. In R. Josselson (Ed.), Ethics and process in the narrative study of lives (p. 172-186). Thousand Oaks, CA: Sage.

Lieblich, A., Tuval-Mashiach, R., \& Zilber, T. (1998). Narrative research: Reading, analysis and interpretation. Thousand Oaks, London and New Delhi.

Like, R.C. (2011). Educating clinicians about cultural competence and disparities in health and health care. Journal of Continuing Education in the Health Professions, 31(3), 196-206. doi: 10.1002/chp.20127 
Lipson J.G., \& DeSantis, L.A. (2007). Current approaches to integrating elements of cultural competence in nursing education. Journal of Transcultural Nursing, 18(1), 10S-20S. doi: $10.1177 / 1043659606295498$

Long, T.B. (2012). Overview of teaching strategies for cultural competence in nursing students. Journal of Cultural Diversity, 19(3), 102-108.

Lowe, J., \& Archibald, C. (2009). Cultural diversity: The intention of nursing. Nursing Forum, 44, 11-18.

Mac Avoy , S. \& Troth Lippman, D. (2001). Teaching culturally competent care: Nursing students experience in rural Applachia. Journal of Transcultural Nursing, 12, 221227.

Maddalena, V. (2009). Cultural competence and holistic practice: Implications for nursing education, practice, and research. Holistic Nursing Practice, 23, 153-157. doi:10.1097/HNP.0b013e3181a056a0.

Majumdar, B., Browne, G., Roberts, J., \& Carpio, B. (2004). Effects of cultural sensitivity training on health care provider attitudes and patient outcomes. Journal of Nursing Scholarship, 36(2), 161-166. doi: 10.1111/j.1547-5069.2004.04029.x

Manitoba Historical Society, (2015). Memorable Manitobans: Paul Yuzyk (1913-1986). Retrieved from http://www.mhs.mb.ca/docs/people/yuzyk_p.shtml Mattson, S., 2000. Providing culturally competent care. Lifelines October/November 3739.

Markova, T., \& Broome, B. (2007). Effective communication and delivery of culturally competent health care. Urology Nursing, 27:239-242. 
McBrien, J.L. \& Day, R. (2012). From there to here: Using photography to explore perspectives of resettled refugee youth. International Journal of Child, Youth and Family Studies, 4(1), 546-568.

McCance, T., McKenna, H., \& Boore, J. (2001). Exploring caring using narrative methodology: An analysis of the approach. Journal of Advanced Nursing, 39, 96102.

McEwen, M., \& Wills, E.M. (2011). Theoretical basis for nursing (3 ${ }^{\text {rd }}$ ed.). Lippincott Williams \& Wilkins.

McGinnis, S.L., Brush, B.L., \& Moore, J. (2010). Cultural similarity, cultural competence, and nurse workforce diversity. Western Journal of Nursing Research, 32(7), 894-909.

Menec, V.H., Shooshtari, S., \& Lambert, P. (2007). Ethnic differences in self-rated health among older adults: A cross-sectional and longitudinal analysis. Journal of Aging and Health, 19(1), 62-86. doi: 10.1177/0890264306296397

Merriam-Webster Online. (2015). Need. Retrieved from http://www.merriam-webster.com/dictionary/need Ministry of Health and Long-Term Care, (2010). Long-Term Care Homes Act. Retrieved from http://www.ontario.ca/laws/regulation/r10079

Ministry of Health and Long-Term Care, (2011). Seniors' care: Overview of care options. Retrieved from http://www.health.gov.on.ca/en/public/programs/ltc/ Mishler, E. (1986). Research interviewing: Context and narrative. Cambridge: Harvard University Press. 
Mold, F., Fitzpatrick, J. M., \& Roberts, J. D. (2005). Minority ethnic elders in care homes: A review of the literature. Age and Ageing, 34, 107-113.

Morse, J.M., Hupcey, J.E., Mitcham, C., \& Lenz, E.R. (1996). Concept analysis in nursing research: A critical appraisal. Scholarly Inquiry for Nursing Practice, $10(3), 253-277$.

Mullings, D.V., \& Gien, L. (2013). Culturally competent (appropriate) health and longterm care services for older immigrants in a small urban center of Newfoundland. International Journal of Humanities and Social Science Invention, 2(9), 81-89.

Munhall, P.L. (2012). Nursing research: A qualitative perspective (5 $5^{\text {th }}$ ed.). Sudbury, MA: Jones \& Bartlett Learning.

Murphy, S. (2006). Mapping the literature of transcultural nursing. Journal of the Medical Library Association of North America, 94, (2), E143-E151.

Newbold, K.B., \& Danforth, J. (2003). Health status and Canada's immigrant population. Social Science \& Medicine, 57(10), p. 1981-1995. doi: 10.1016/S0277$9536(03) 000649$

Ng, E., Lai, D.W.K., Rudner, A.T., \& Orpana, H. (2012). Working Paper Series: What do we know about immigrant seniors aging in Canada? A demographic, socioeconomic and health profile. Retrieved from: http://aaisa.ca/wpcontent/uploads/2013/02/What-do-we-know-about-immigrant-seniors-aging-inCanada1.pdf 
Ngo-Metzger, Q., Telfair, J., Sorkin, D.H., Weidmer, B., Weech-Maldonado, R., Hurtado, M., \& Hays, R.D. (2006). Cultural competency and quality of care: Obtaining the patient's perspective. Retrieved from http://www.commonwealthfund.org/publications/fundreports/2006/oct/cultural- competency-and-quality-of-care--obtaining-thepatients-perspective

Nizum, N. (2015). Exploring experiences of challenge and resilience in South Asian immigrant older adults living with mild dementia in the Greater Toronto Area. Toronto, Canada: Ryerson University

Nyatange, B, \& de Vocht, H. (2008). Intuition in clinical decision-making: A psychological penumbra. International Journal of Palliative Care, 14(10), 1-5.

Ontario Federation of Community Mental Health and Addiction Programs. (2009). Embracing Cultural Competence in Mental Health and Addiction System. Retrieved from http://fpyn.ca/resource/cultural-competence-and-mental-health-andaddictions-system

Ontario Ministry of Finance. (June 2015). Ontario Fact Sheet July 2015. Retrieved from http://www.fin.gov.on.ca/en/economy/ecupdates/factsheet.html

Ortiz, F., \& Fitten, L. J. (2000). Barriers to healthcare access for cognitively impaired older Hispanics. Alzheimer Disease \& Associated Disorders, 14\{3), 141-15.

Ortlipp, M. (2008). Keeping and using reflective journals in the qualitative research process. The Qualitative Report, 13(4), 695-705.

Parker, I. (2005). Qualitative psychology: Introducing radical research. Buckingham, UK: Open University Press. 
Parker, V.A., \& Geron, S.M. (2007). Cultural competence in nursing homes: Issues and implications for education. Gerontology \& Geriatrics Education, 28(2), 37-54. doi: 10.1300/J021v28n02_04

Pecukonis, E., Doyle, O., \& Bliss, D.L. (2008). Reducing barriers to interprofessional training: Promoting interprofessional cultural competence. Journal of Interprofessional Care, 22(4), 417-428.

Perloff, R., Bonder, B., Ray, G., Ray, E., \& Siminoff, L. (2006). Doctor-patient communication, cultural competence, and minority health: Theoretical and empirical perspectives. American Behavioral Scientist, 49, 835-852.

Petrov, L., \& Arnold, L. (2000). Special needs, special wants. Australasian Journal on Aging, 19, 43-44. doi:10.1111/j.1741-6612.2000. tb00138.x

Phillips, K.L., Chiriboga, D.A., \& Jang, Y. (2012). Patients' perceptions of the interpersonal sensitivity of their healthcare providers: The potential role of patient-provider racial/ethnic concordance. Patient, 5(3), 175-183.

Pitkin Derose, K., Escarce, J.J., \& Lurie, N. (2007). Immigrants and health care: Sources of vulnerability. Health Affairs, 25(5), 1258-1268. doi: 10.1377/hlthaff.26.5.1258

Ponce, N. A., Hays, R. D., \& Cunningham, W. E. (2006). Linguistic disparities in health care access and health status among older adults. Journal of General Internal Medicine, 21, 786-791.

Pretz, J.E. \& Folse, V.N. (2011). Nursing experience and preference for intuition in decision making. Journal of Clinical Nursing, 20(19-20), 2878-2889. doi: $10.1111 / \mathrm{j} .1365-2702.2011 .03705 . \mathrm{x}$ 
Pretz, J.E., \& Totz, K.S. (2007). Measuring individual differences in affective, heuristic, and holistic intuition. Personality and Individual Differences, 43, 1247-1257.

Purnell, L. (2002). The Purnell Model for Cultural Competence. Journal of Transcultural Nursing, 13, 193-196. doi: 10.1177/10459602013003006

Regev, O.E. (2014). Cultural competence through nurses' narratives: A qualitative research of hospital nurses in Israel. Revista De Asstenta Sociala, 8(2), 51-66.

Registered Nurses' Association of Ontario, (2007). Embracing cultural diversity in health care: Developing cultural competence. Toronto: Registered Nurses Association of Ontario.

Reimer Kirkham, S. (1998). Nurses' descriptions of caring for culturally diverse clients. Clinical Nursing Research, 7(2), 125-146.

Renzaho, A.M.N., Romios, P., Crock, C., \& Sonderlund, A.L. (2013). The effectiveness of cultural competence programs in ethnic minority patient-centered health care: A systematic review of the literature. International Journal for Quality in Health Care, 25(3), 261-269.

Riessman, C.K. (1993). Narrative analysis (Vol. 30). Thousand Oaks, CA: Sage. Riessman, C.K. (2008). Narrative methods for the human sciences. California: SAGE Publications, Inc.

Robinson, G.R., \& Gallagher, A. (2008). Culture change impacts quality of life for nursing home residents. Topics in Clinical Nutrition, 23(2), 120-130.

Romanow, R.J. (2002). Building on Values: The Future of Health Care in Canada. Retrieved from: http://publications.gc.ca/collections/Collection/CP32-852002E.pdf 
Rumay Alexander, G. (2008). Cultural competence models in nursing. Critical Care Nursing Clinics of North America, 20, 415-421.

Runci, S.J., Eppingstall, B.J., van der Ploeg, E.S., \& O’Connor, D.W. (2014).

Comparison of family satisfaction in Australian ethno-specific and mainstream aged care facilities. Journal of Gerontological Nursing, 40(4), 55-63.

Ryan, F., Coughlan, M. , \& Cronin, P. (2009) Interviewing in qualitative research: the one-to-one interview. International Journal of Therapy and Rehabilitation 16(6), 309-314.

Saha, S., Arbelaez, J., \& Cooper, L. (2003). Patient-physician relationships and racial disparities in the quality of health care. American Journal of Public Health, 93(10), 1713-1719.

Sanchez, C. A. (2008). Cultural (Incompetence), Justice, and Expectations of Care: An Illustration. Online Journal of Health Ethics, 5(1). Retrieved from http://aquila.usm.edu/ojhe/vol5/iss1/

Sargent, S., Sedlak, C., \& Martsolf, D. (2005). Cultural competence among nursing students and faculty. Nurse Education Today, 25, 214-221.

doi:10.1016/j.nedt.2004.12.005.

Schim, S.M., Doorenbos, A., Benkert, R., \& Miller, J. (2007). Culturally congruent care: Putting the Puzzle Together. Journal of Transcultural Nursing, 18(2), 103-110. doi:10.1177/1043659606298613

Schim, M. S., Doorenbos, A.Z., \& Borse, N.N. (2005). Cultural competence among Ontario and Michigan healthcare providers. Journal of Nursing Scholarship, 37(4), 354-360. 
Seaton, L.P. (2010). Cultural care in nursing: A critical analysis. Retrieved from: http://www.researchgate.net/profile/Lesley_Seaton/publication/265496391_CUL TURAL_CARE_IN_NURSING_A_CRITICAL_ANALYSIS/links/55027c280cf 24cee39fc1def.pdf

Shen, Z. (2004). Cultural competence models in nursing: A selected annotated bibliography. Journal of Transcultural Nursing, 15, 317-322.

Sorensen, G., Barbeau, E., Stoddard, A.M., Hunt, M.K., Kaphingst, K., Wallace, L. (2005). Promoting behavior change among working class, multiethnic workers: Results of the healthy directions - small business study. American Journal of Public Health, 95, 1389-1395. doi: 10.2105/AJPH. 2004.038745.

Starr, S., Shattell, M. M., \& Gonzales, C. (2011). Do nurse educators feel competent to teach cultural competency? Teaching and Learning Nursing, 6, 84-88. doi:10.1016/j .teln.2010.12.004

Starr, S. \& Wallace, D.C. (2009). Self-reported cultural competence of public health nurses in a southeastern U.S. public health department. Public Health Nursing 26, $48-57$.

Statistics Canada. (2014). Canada's population estimates: Age and sex, 2014. Retrieved from http://www.statcan.gc.ca/daily-quotidien/140926/dq140926b-eng.htm Statistics Canada. (2013). Living arrangements of seniors. Retrieved from http://www12.statcan.ca/census-recensement/2011/as-sa/98-312-x/98-312x2011003_4-eng.cfm 
Stewart, M., Shizha, E., Makwarimba, E., Spinzer, D., Khalema, E.N., \& Nsaliwa, C.D. (2011). Challenges and barriers to services for immigrant seniors in Canada: "You are among others but you feel alone". International Journal of Migration, Health and Social Care, 70(1), 16-32. doi:10.1108/1747989111116278

Streubert, H.J. \& Carpenter, D.R. (2011). Qualitative research in nursing: Advancing the humanistic imperative ( $5^{\mathrm{TH}}$ ed.). Lippincott Williams \& Wilkins.

Stuart, L., \& Nielsen, A-M. (2011). Two Aboriginal Registered Nurses show us why black nurses caring for black patients is good medicine. Contemporary Nurse, 37(1), 96-101.

Sue, D.W., \& Sue, D. (2003). Counseling the culturally diverse: Theory and practice. New York: Wiley.

Suhonen, R., Alikleemola, P., Katajisto, J., \& Leino-Kilpi, H. (2011). Nurses’ assessment of individualized care in long-term care institutions. Journal of Clinical Nursing, 21, 1178-1188. doi: 10.1111/j.1365-2702.2011.03855.x

Suhonen, R., Valimaki, M., \& Leino-Kilpi, H. (2008). Outcomes of individualized nursing interventions on adult patients: A literature review. Journal of Clinical Nursing, 18, 843-860.

Sumpter, D., \& Carthon, J.M. (2011). Lost in translation: Student perceptions of cultural competence in undergraduate and graduate nursing curricula. Journal of Professional Nursing, 27(1), 43-49. doi: 10.1016/j.profnurs.2010.09.005

Tabari-Khomeiran, R., Kiger, A., Parsa-Yekta, Z., \& Ahmadi, F. (2007). Competence development among nurses: The process of constant interaction. The Journal of Continuing Education in Nursing, 38(5), 211-218. 
Tayab, A., \& Narushima, M. (2015). "Here for the Residents": A case study of cultural competence of personal support workers in a long-term care home. Journal of Transcultural Nursing, 26(2), 146-156.

Taylor, R. (2005). Addressing barriers to cultural competence. Journal for Nurses in Staff Development, 21(4), 135-142.

Taylor, R.A., \& Alfred, M. (2010). Nurses' perceptions of the organizational supports needed for the delivery of culturally competent care. Western Journal of Nursing Research, 32(5), 591-609.

Tellis-Nayak V. (2007). A person-centered workplace: the foundation for personcentered caregiving in long- term care. Journal of American Medical Directors Association, 8(1), 45-54.

Tofthagen, R., \& Fagerstrom, L.M. (2010). Rodgers' evolutionary concept analysis: A valid method for developing knowledge in nursing science. Scandinavian Journal of Caring Sciences, 24, 21-31.

Toronto Ukrainian Genealogy Group, (2005). The history of Ukrainians in Canada. Retrieved from http://www.torugg.org/History/history_of_ukrainians_in_canada.html Tortumluoglu, G. (2006). The implications of transcultural nursing models in the provision of culturally competent care. ICUS Nursing Web Journal, 25(JanuaryApril), 1-11.

Tri-Council Policy Statement 2. (2014). Ethical Conduct for Research Involving Humans. Retrieved from http://www.pre.ethics.gc.ca/pdf/eng/tcps22014/TCPS_2_FINAL_Web.pdf 
Tucker, C.M., Marsiske, M., Rice, K.G., Jones, J.D., \& Herman, K.C. (2011). Patientcentered culturally sensitive health care: Model testing and refinement. Health Psychology, 30(3), 342-350.

Twinn, S. (1997). An exploratory study examining the influence of translation on the validity and reliability of qualitative data in nursing research. Journal of Advanced Nursing, 26, 418-423. Retrieved from http://journals2.scholarsportal.info.ezproxy.lib.ryerson.ca/tmp/158580362653789 21067.pdf

Ukrainian Canadian Congress. (2012). Ontario. Retrieved from http://www.ucc.ca/members/provincial-councils/ontario/

Ujimoto, K.V. (1994). Racial and ethnic dimensions of aging: Implications for health care services. (pg. 201-224) as printed in Racial Minorities Medicine and Health Bolaria, B.S., \& Bolaria, R.). Fernwood Publishing, Halifax, Nova Scotia.

Ulrey, K.L., \& Amason, P. (2001). Intercultural communication between patients and health care providers: an exploration of intercultural communication effectiveness, cultural sensitivity, stress, and anxiety. Health Communication, 13(4), 449-463.

Van-Ryn, M., \& Burke, J. (2000). The effect of patient race and socio-economic status on physicians' perceptions of patients. Social Science and Medicine, 50(6), 813-828.

Walsh, L. V. \& DeJoseph, J. (2003). "I saw it in a different light". International learning experiences in baccalaureate nursing education. Journal of Nursing Education, $42,266-272$. 
Waxler-Morrison, N., Anderson, J.M., Richardson, E., \& Chambers, N.A. (2005). Crosscultural caring: A handbook for health professionals ( $2^{\text {nd }}$ ed.). Vancouver: UBC Press.

Weinick, R.M., Zuvekas, S.H., 2000. Racial and ethnic differences in access to and use of healthcare services. Medical Care Resident Review, 57, 36-54.

Williams, C. (2002). Evaluation of an educational intervention to increase cultural competence in social workers. Unpublished Doctoral Dissertation, University of Toronto, Toronto, Ontario, Canada.

Williamson, M., \& Harrison, L. (2010). Providing culturally appropriate care: A literature review. International Journal of Nursing Studies, 47, 761-769. doi:10.1016/j.ijnurstu.2009.12.012

Wister, A., Pereira, I., Lazarowich, N. (1996). Ethnic content in long-term care facilities for Portuguese and Italian elderly. Canadian Ethnic Studies. 28(2). 82-97.

Woodhouse, J. (2012). The use of visual methodology in nursing. Nursing Researcher, 19(3), 20-25.

Woolley, A., \& Kostopoulou, O. (2013). Clinical intuition in family medicine: More than first impressions. Annals of Family Medicine, 11(1), 60-66.

Wysong, P.R., \& Driver, E. (2009). Patients' perceptions of nurses' skill. Critical Care Nurse, 29, 24-37.

Yeboah, C., Bowers, B., \& Rolls, C. (2013). Culturally and linguistically diverse older adults relocating to residential aged care. Contemporary Nurse, 44(1), 50-61. 
Zane, N., Sue, S., Chang, J., Huang, L., Huang, J., \& Lowe, S., et al. (2005). Beyond ethnic match: Effects of client-therapist cognitive match in problem perception, coping orientation, and therapy goals on treatment outcomes. Journal of Community Psychology, 33(5), 569-585.

Zhang, Z., Luk, W., Arthur, D., \& Wong, T. (2001). Nursing competencies: Personal characteristics contributing to effective nursing performance. Issues and Innovations in Nursing Practice, 33(4), 467-474. 


\section{GLOSSARY OF TERMS}

Culture. A shared set of beliefs, ideas and behaviors associated with membership within a group defined by socioeconomic status, ethnic or racial origins, language similarity or religious groundings. Culture influences how people behave and make sense of the world, as it is an inherent lens for considering one's environment. This definition is based on Leininger's (1991) Transcultural Theory of Nursing.

Cultural Competence. In healthcare, this concept refers to the ability of healthcare professionals to work effectively within the cultural context of the clients. The development of cultural competence is an ongoing process. The individual transitions from having limited understanding of the client's cultural healthcare needs to expert ability to integrate cultural awareness, cultural knowledge, cultural skill and cultural encounters, the attributes of cultural competence, into the delivery of culturally competent care to clients from culturally diverse backgrounds. The starting point of development of cultural competence is frequently cultural desire, or the voluntary willingness to learn. The attributes of cultural competence introduced in this definition stem from Campinha-Bacote's (2002) model titled Process of Cultural Competence in the Delivery of Healthcare Services. Cultural competence can exists at the level of individual healthcare providers, as well as at the level of healthcare and government organizations.

Culturally Competent Care. As the moral and ethical responsibility of healthcare professionals and organizations, culturally competent care refers to the care approach that promotes cross-cultural equality of clients, incorporates transcultural knowledge and demonstrates respect for cultural practices of clients (College of Nurses on Ontario, 2009; Dudas, 2012; Mattson, 2000). It is commonly associated with individualized care. Benefits of culturally competent care include improved communication between healthcare providers and clients, as well as overall enhanced health outcomes of care recipients (Betancourt, Green, Carillo, \& park, 2005; Like, 2011).

Culturally Competent Nurse. A nurse who consistently demonstrates respect for and sensitivity to the clients' culturally-oriented needs. She has the skills, knowledge and attitudes associated with cultural competence, and the capacity to integrate the client's cultural context into her care so that the needs of the individual are appropriately met. This definition is an integration of Boi's (2000) research of the nurses' perceptions of what it means to provide culturally competent care and the views of healthcare clients on the defining characteristics of a skillful nurse discussed by Wysong and Driver (2009).

Cultural Incompetence. Cultural incompetence corresponds to the initial stages of cultural competence development process when the individual is fully devoid of cultural sensitivity. Through literature review, it has been inferred that cultural incompetence predominates during the first three stages of cultural competence development identified by Cross, Barzon, Dennis and Isaacs (1989), namely during the stages of cultural destructiveness, cultural incapacity and cultural blindness. Cultural incompetence is associated with ethnocentric outlook, the tendency to stereotype and overall restricted capacity to provide culturally competent care. 
Cultural Insight. As an emergent concept, cultural insight refers to the inherent understanding one holds of the essence of their culture as the result of having been brought up in the traditions of the group. The definition of cultural insight comes from the analysis of the narratives of the two participants of this study; as such, the definition will benefit from further scholarly exploration. It is, however, expected that capacity of healthcare providers, in particular nurses, to provide culturally competent care of the highest quality to individuals with whom they share a common cultural background is strengthened through cultural insight.

Culturally-Specific Long-term care Home. Institutional living facilities that are recognized as promoting elements of a specific culture through the physical features, social environment and workforce composition of the home (Barrass, 2012). As healthcare settings, they provide support and varying degrees of assistance with activities of daily living for the residents (Community Care Access Centre, 2015). In colloquial terms, this definition refers to nursing homes (i.e. Ukrainian-specific nursing home).

Ethnic Match. As an outcome of individualized approach to care, ethnic match refers to the practice of pairing healthcare providers and healthcare recipients based on a common cultural background, defined as ethnic or racial origins, language congruency or similar religious affiliations (Gilchrist \& Rector, 2007). It is anticipated that such a match will preemptively create trust, minimize communication barriers and improve understanding within the provider-client dyad, thus resulting in better health outcomes for the clients (Benkert et al., 2006; Pope-Davis et al., 2002; Stuart \& Nielsen, 2011). Intra-cultural and inter-generational variations in expression and understanding of culture have been identified as potential barriers to the effectiveness of ethnic match (Waxler-Morrison et al., 2005). Much of the current literature on the practice of ethnic match centers on physician-patient relationships (Saha, Beach, \& Cooper, 2008).

Ethnocentrism. The term ethnocentrism describes attitudes, beliefs and behaviors - a collective life perspective - that reflect one's conviction of the superiority of their own culture, while demonstrating disregard for the unique cultural background of others (Capell, Dean, \& Veenstra, 2008). Ethnocentrism has been associated with poor capacity to provide culturally competent care (Capell et al., 2008).

Livinghood - Emerging as an element of importance to culturally competent care from the narrative of Participant Two, the term livinghood refers to the essence of the individual stemming from their cultural background. The unique needs of the individual, intricately interwoven with cultural affiliations, define the individual stages of the person's life. In positioning livinghood within the paradigm of person-centered care, Participant Two explains that the main objective of culturally competent care is to support livinghood.

Older Immigrant Adult. An individual, 65 years of age or older, who, although he permanently resides in Canada, was born in another country (Statistics Canada, 2010, 2013). 
Person-Centered Care. A departure from the traditional Western biomedical model that focuses primarily on the physical needs of the clients, person-centered care philosophy accepts elements external to the physiological well-being of the individual as having an influence on the health of the individual (Cooper Brathwaite, 2006). The contextualized understanding of individuals' healthcare needs has become the basis of restructuring of the healthcare services, in particular of long-term care frameworks (Kitwood, 1997; Robinson \& Gallagher, 2008).

Personhood. The concept arises out of Kitwood's (1997) work on the individualized care approach for people living with dementia. As such, personhood refers to the bestowing of a status onto one person by another, as a means of affirming their identity and value.

Zolota Seredunka. The term is translated into English as "the golden middle". This is an element inferred to be relevant to culturally competent care from the narrative of Participant One of the study. The aim of culturally competent care is to keep the golden middle or to maintain balance. As such, the focus of care should be on facilitating the resident's ability to carry on with the culturally-defined lifestyle the individual has grown accustomed to over the course of their life. Care of the physical needs of the individual are incorporated into the daily routine in a fluid, non-intrusive manner as a way of keeping the golden middle. The nurse's personal understanding of the client's culture bolsters her ability to keep the golden middle in her care by appropriately incorporating the Western biomedical standards with the client's cultural preferences. 